\title{
Ir-Catalyzed Atroposelective Desymmetrization of Heterobiaryls: Hydroarylation of Vinyl Ethers and Bicycloalkenes
}

\author{
Antonio Romero-Arenas, ${ }^{\dagger}$ Valentín Hornillos,${ }^{,+}$Javier Iglesias-Sigüenza, ${ }^{\ddagger}$ Rosario

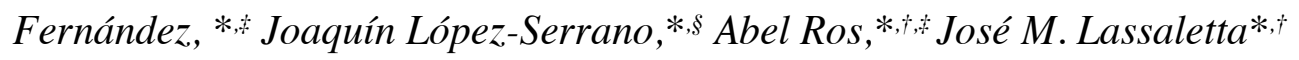

$\dagger$ Instituto de Investigaciones Químicas (CSIC-US) and Centro de Innovación en Química Avanzada (ORFEO-CINQA), Avda. Américo Vespucio, 49, 41092 Sevilla, Spain.

§Instituto de Investigaciones Químicas (CSIC-US). Departamento de Química Inorgánica and Centro de Innovaciones Química Avanzada (ORFEO-CINQA). Avda. Américo Vespucio, 49,41092 Sevilla, Spain.

$\ddagger$ Departamento de Química Orgánica, Universidad de Sevilla and Centro de Innovación en Química Avanzada (ORFEO-CINQA), C/Prof. García González, 1, 41012 Sevilla, Spain 
${ }^{1} \mathrm{H}$ NMR $\left(400 \mathrm{MHz}, \mathrm{CDCl}_{3}\right)$ of $\left(S_{a}, R\right)-\mathbf{7 a}$ :

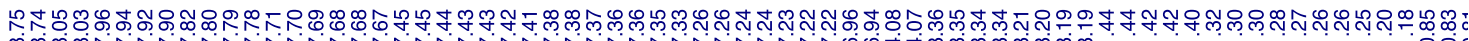

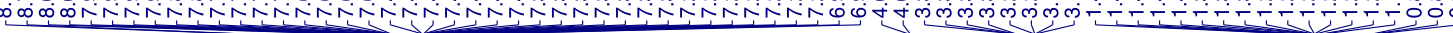

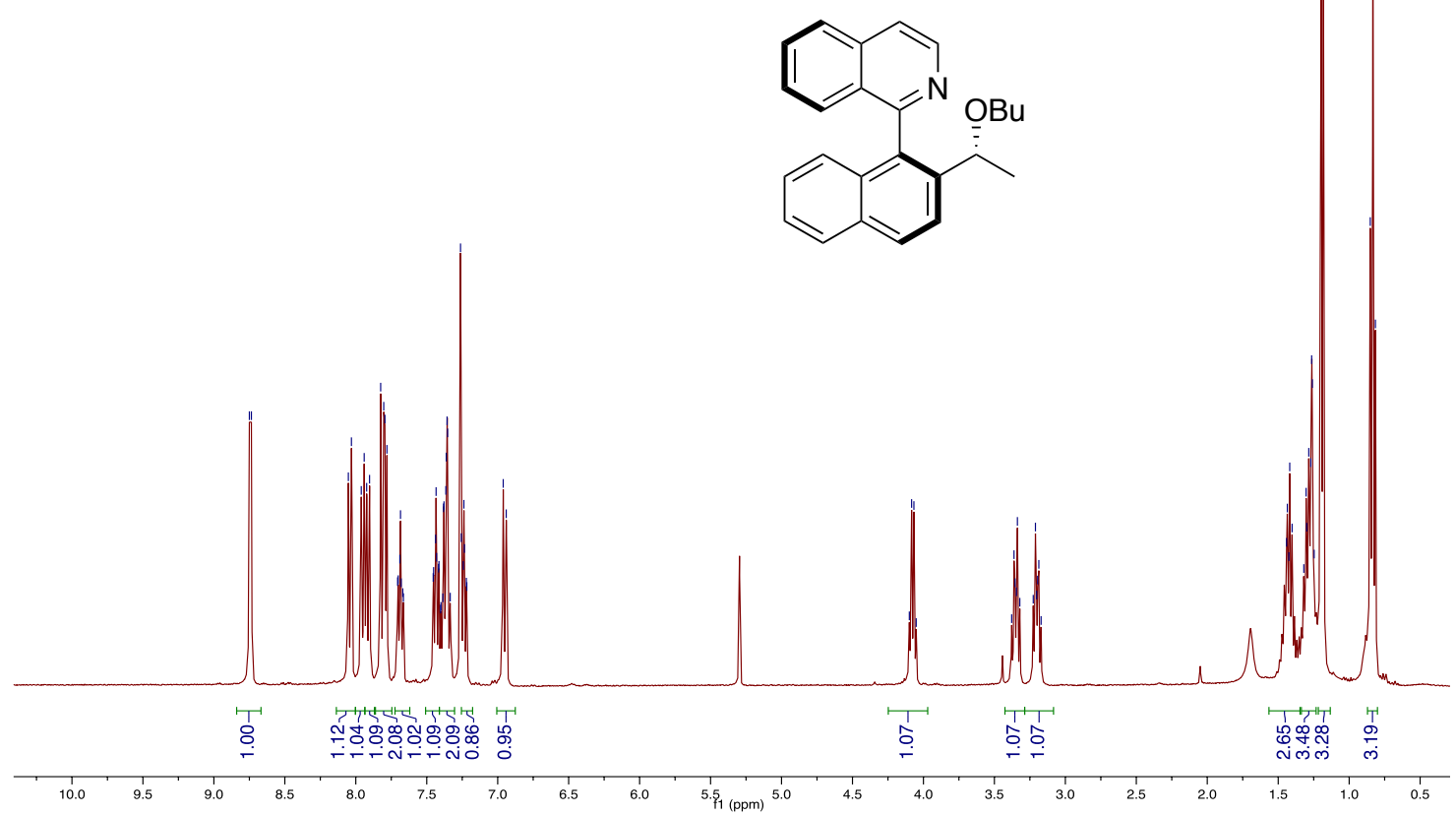

${ }^{13} \mathrm{C}$ NMR $\left(100 \mathrm{MHz}, \mathrm{CDCl}_{3}\right)$ of $\left(S_{a} R\right)-\mathbf{7 a}$ :

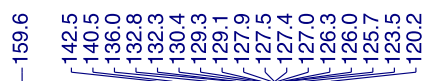

$\begin{array}{lll}\hat{n} & m \\ & \infty & 0 \\ 1 & 0 & 1\end{array}$

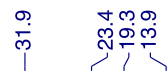<smiles>CC(C)c1ccc2ccccc2c1-c1nccc2ccccc12</smiles>

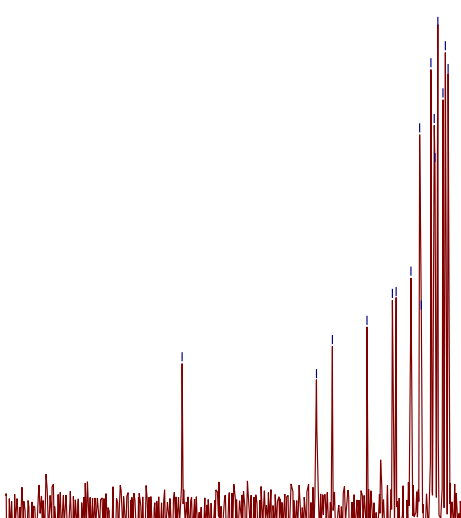

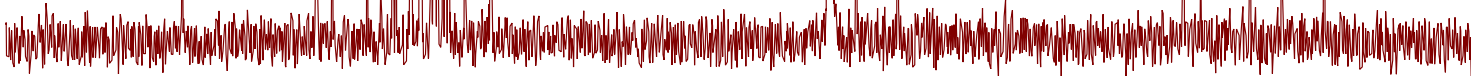

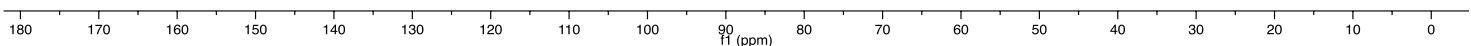


Racemic sample of $( \pm)$-7a: IA column, $n$-hexane:isopropanol 97:3, F= $1 \mathrm{~mL} / \mathrm{min}, \mathrm{T}=$ $30^{\circ} \mathrm{C}$.

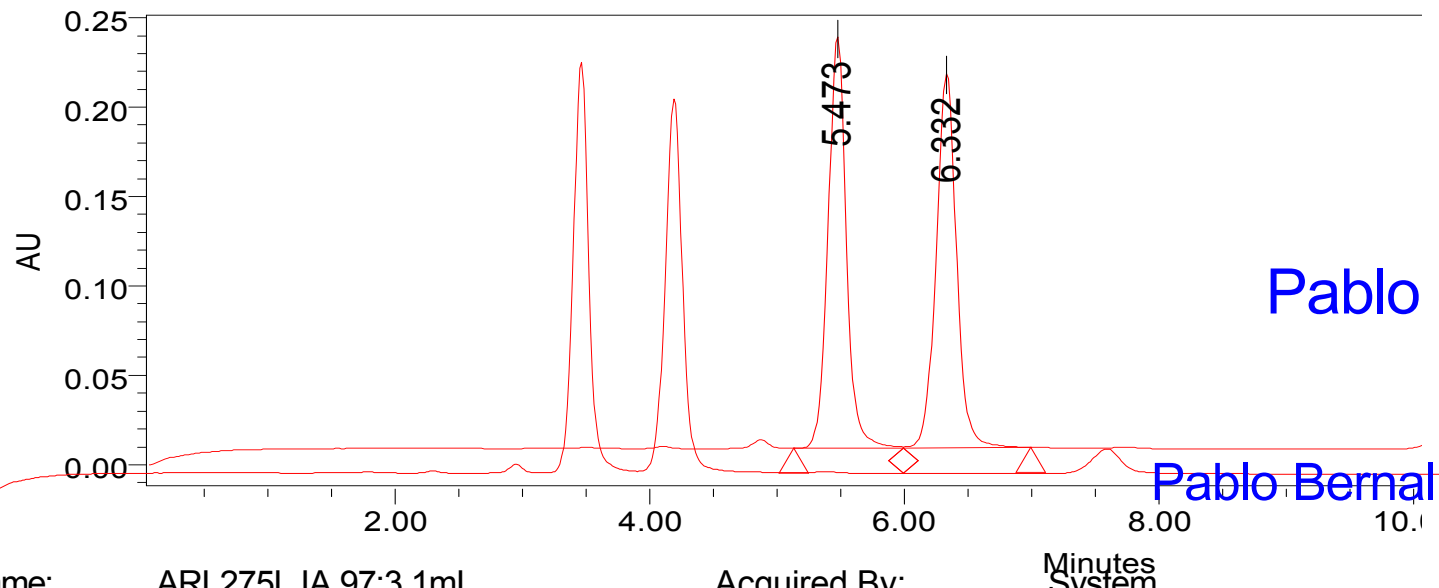

\section{Processed Channel: PDA 220.0 nm}

\begin{tabular}{|l|l|r|r|r|l|}
\hline & Processed Channel & $\begin{array}{r}\text { Retention } \\
\text { Time }(\mathrm{min})\end{array}$ & Area & \% Area & Height \\
\hline 1 & PDA 220.0 nm & 5.473 & 2284828 & 49.56 & 230659 \\
\hline 2 & PDA 220.0 nm & 6.332 & 2325579 & 50.44 & 209447 \\
\hline
\end{tabular}

\section{Enantioriched sample of $\left(S_{a}, R\right)-7 a$ :}

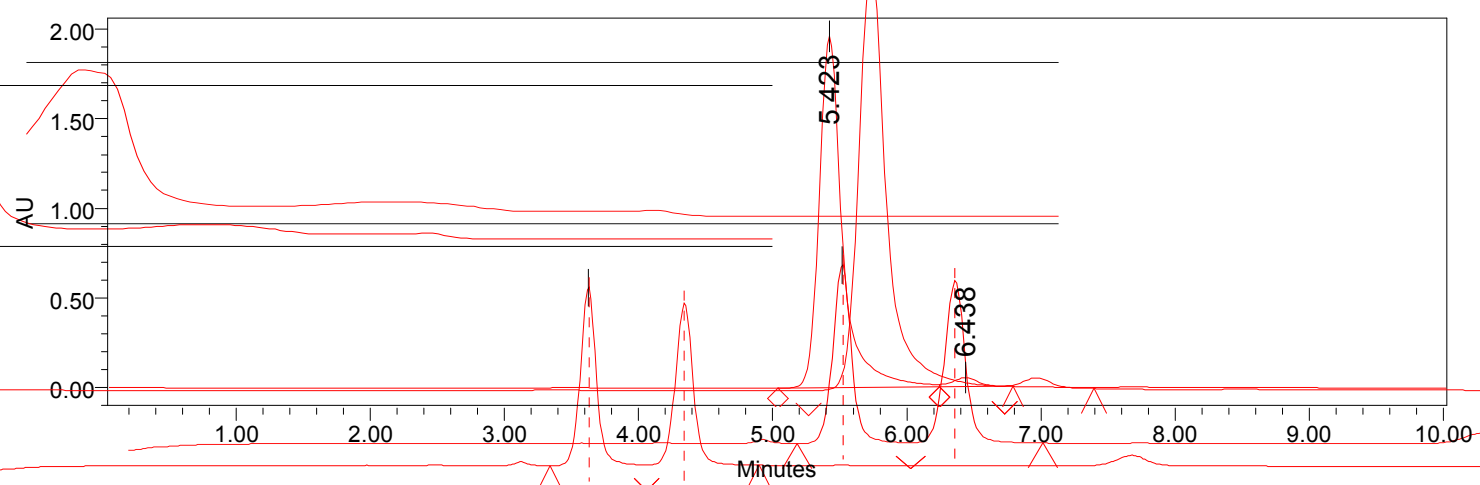

Processed Channel: PDA $223.3 \mathrm{~nm}$

\begin{tabular}{|r|l|r|r|r|r|}
\hline & Processed Channel & $\begin{array}{r}\text { Retention } \\
\text { Time }(\mathrm{min})\end{array}$ & Area & \% Area & Height \\
\hline 1 & PDA 223.3 nm & 5.423 & 23912618 & 97.13 & 1960585 \\
\hline 2 & PDA 223.3 nm & 6.438 & 707077 & 2.87 & 51961 \\
\hline
\end{tabular}


${ }^{1} \mathrm{H}$ NMR $\left(400 \mathrm{MHz}, \mathrm{CDCl}_{3}\right)$ of $\left(S_{a}, R\right)-7 \mathbf{b}$ :

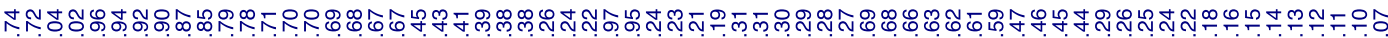

作
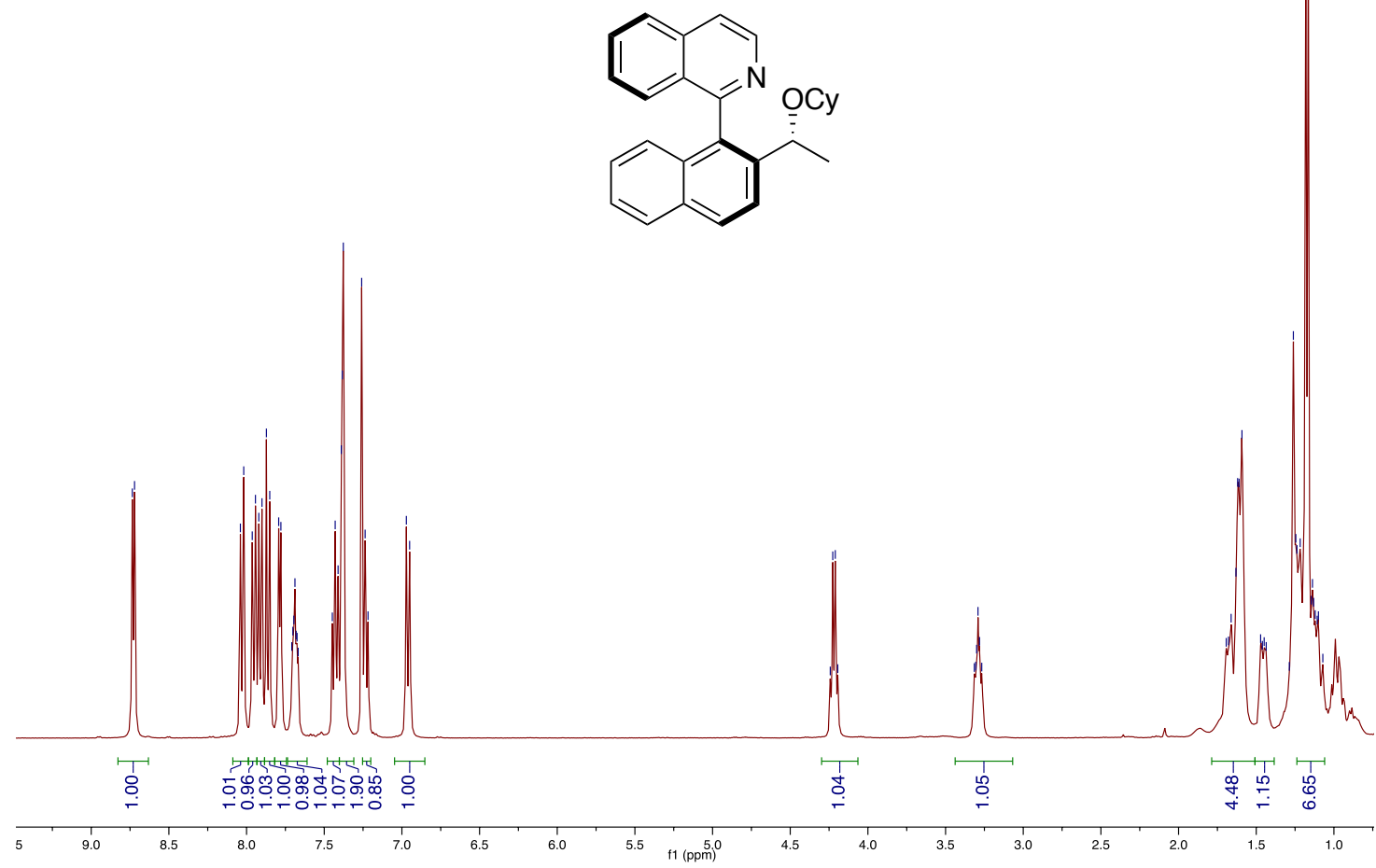

${ }^{13} \mathrm{C}$ NMR $\left(100 \mathrm{MHz}, \mathrm{CDCl}_{3}\right)$ of $\left(S_{a}, R\right)-7 \mathbf{b}$ :

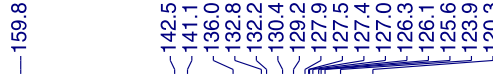

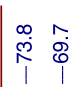

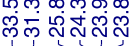
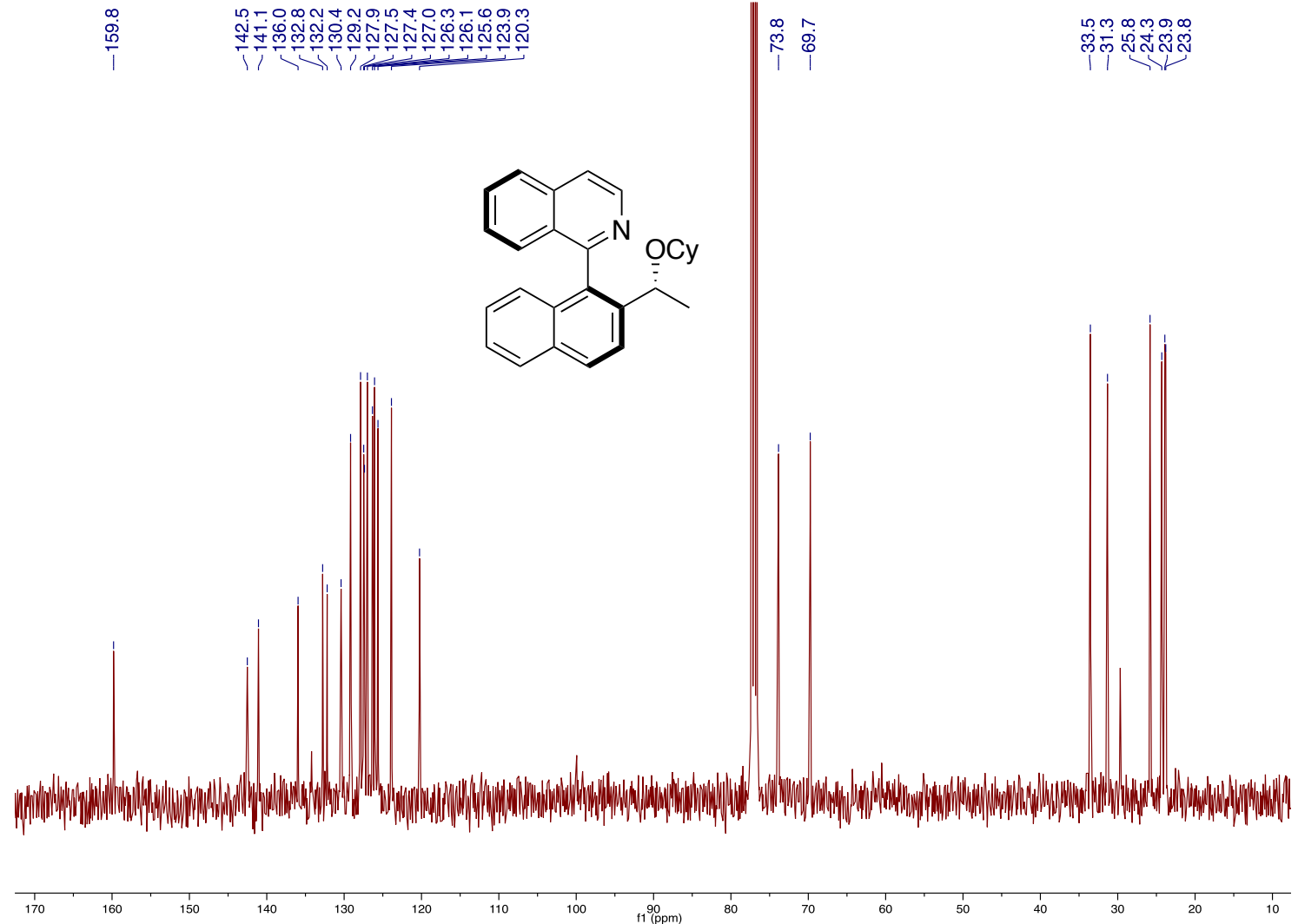
Racemic sample of $( \pm)$-7b: IA column, $n$-hexane:isopropanol 99:1, F= $1 \mathrm{~mL} / \mathrm{min}, \mathrm{T}=$ $30^{\circ} \mathrm{C}$.

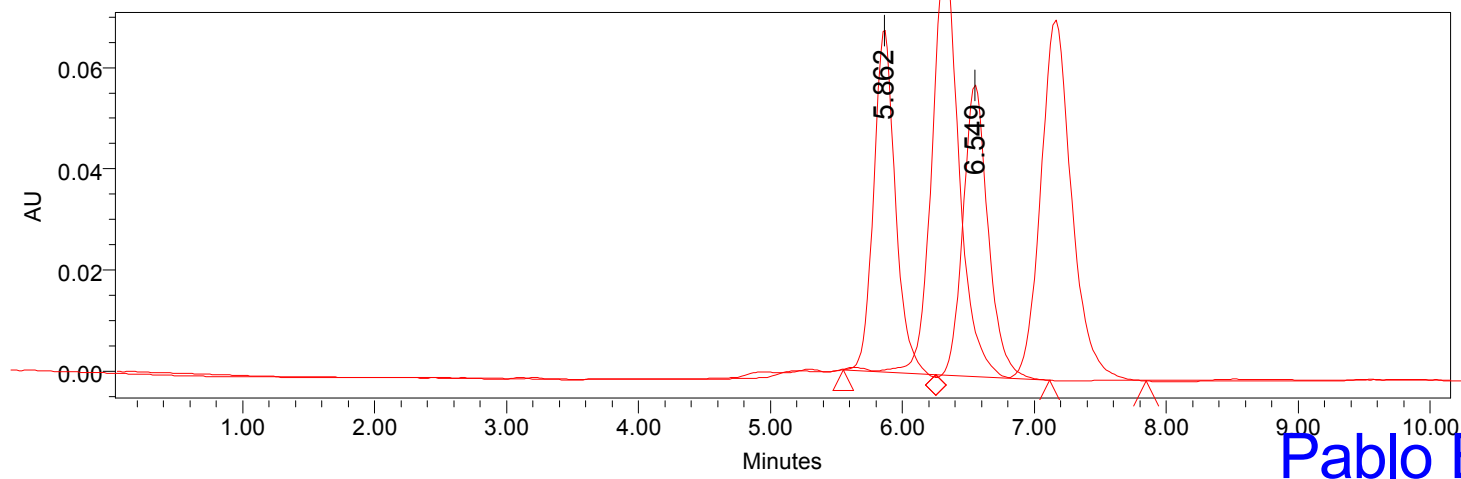

\section{Processed Channel: PDA 218.3 nm}

\begin{tabular}{|l|l|r|r|r|r|}
\hline & Processed Channel & $\begin{array}{c}\text { Retention } \\
\text { Time (min) }\end{array}$ & Area & \% Area & Height \\
\hline 1 & PDA 218.3 nm & 5.862 & 772250 & 50.40 & 67810 \\
\hline 2 & PDA 218.3 nm & 6.549 & 760068 & 49.60 & 57670 \\
\hline
\end{tabular}

Enantioriched sample of $\left(S_{a}, R\right)-7 \mathbf{b}$ :

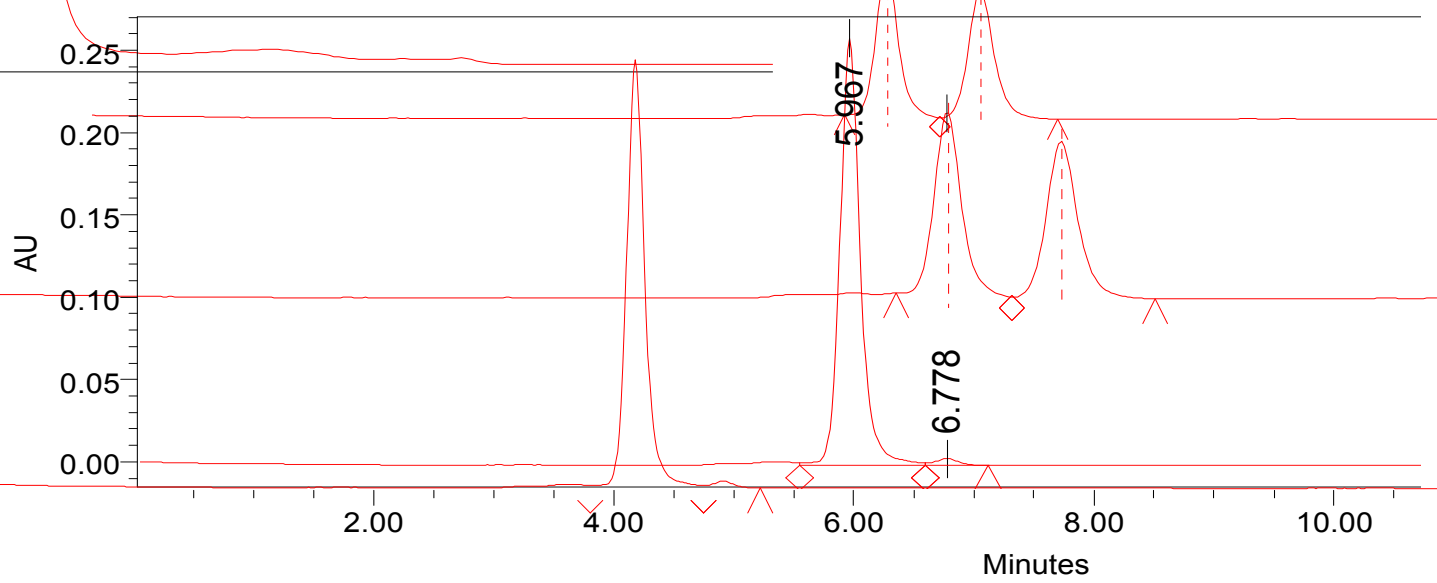

Processed Channel: PDA $220.0 \mathrm{~nm}$

\begin{tabular}{|r|l|r|r|r|r|}
\hline & Processed Channel & $\begin{array}{r}\text { Retention } \\
\text { Time }(\mathrm{min})\end{array}$ & Area & \% Area & Height \\
\hline 1 & PDA 220.0 nm & 5.967 & 3088390 & 98.22 & 259244 \\
\hline 2 & PDA 220.0 nm & 6.778 & 55974 & 1.78 & 4085 \\
\hline
\end{tabular}


${ }^{1} \mathrm{H}$ NMR $\left(400 \mathrm{MHz}, \mathrm{CDCl}_{3}\right)$ of $\left(S_{a}, R\right)-7 \mathbf{c}$ :

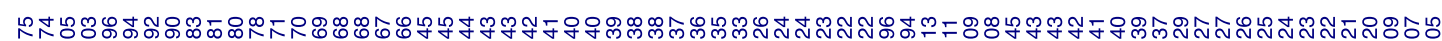

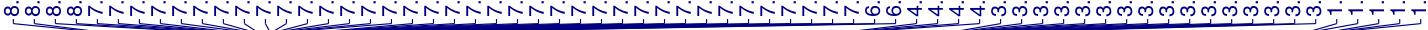

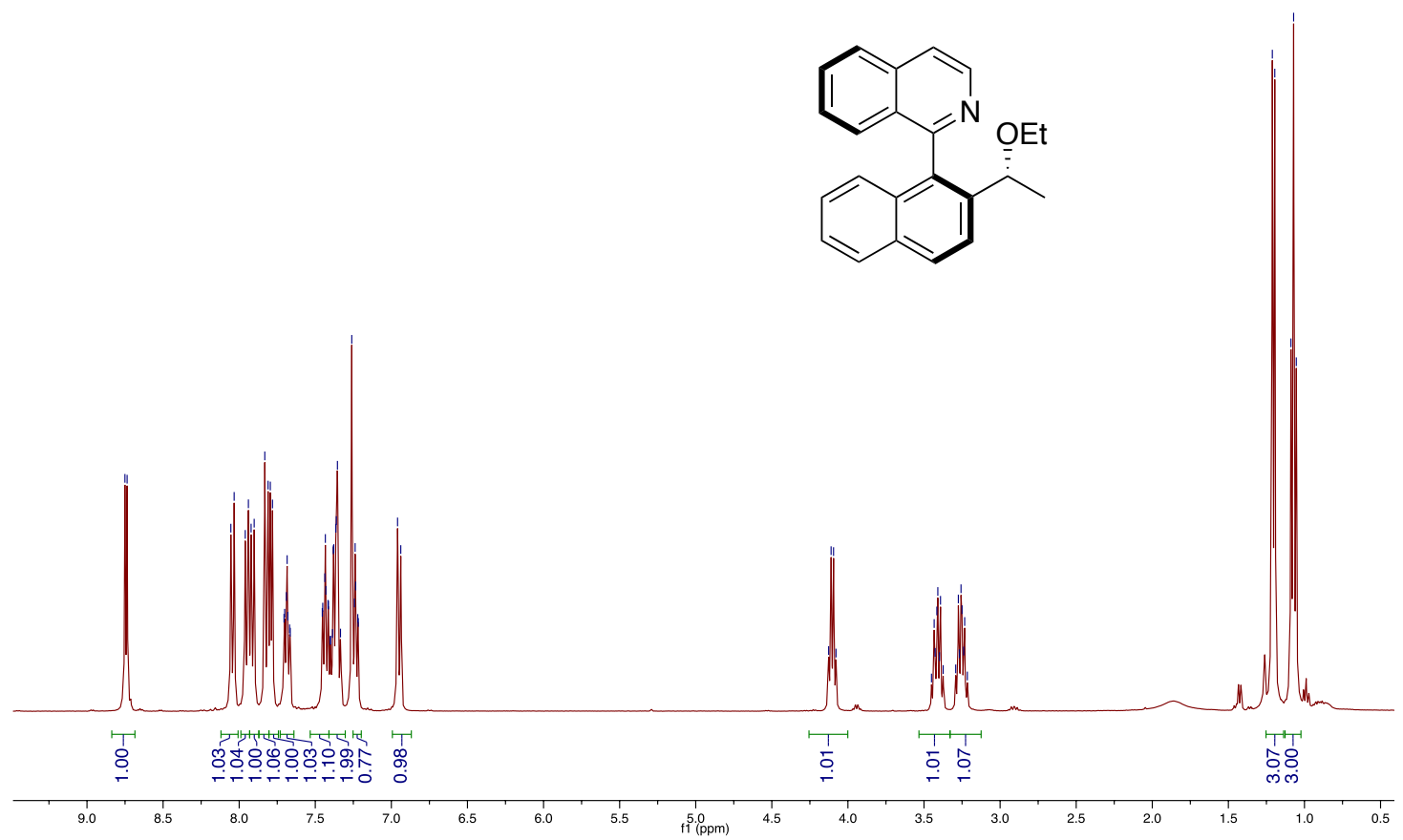

${ }^{13} \mathrm{C}$ NMR $\left(100 \mathrm{MHz}, \mathrm{CDCl}_{3}\right)$ of $\left(S_{a}, R\right)-7 \mathrm{c}$ :

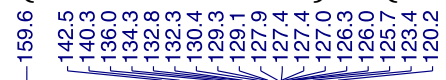

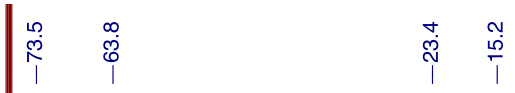

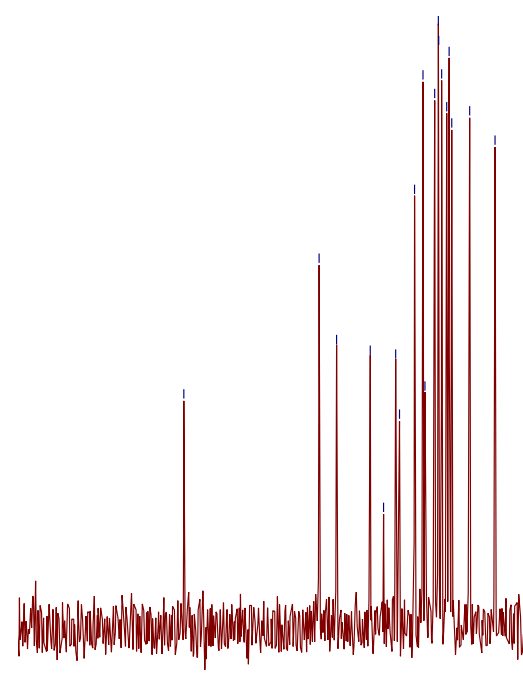<smiles>CCO[C@@H](C)c1ccc2ccccc2c1-c1nccc2ccccc12</smiles>

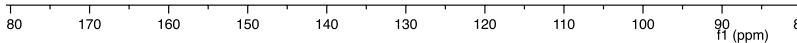


Racemic sample of $( \pm)-7$ c: IA column, $n$-hexane:isopropanol 97:3, F= $1 \mathrm{~mL} / \mathrm{min}, \mathrm{T}=$ $30^{\circ} \mathrm{C}$.

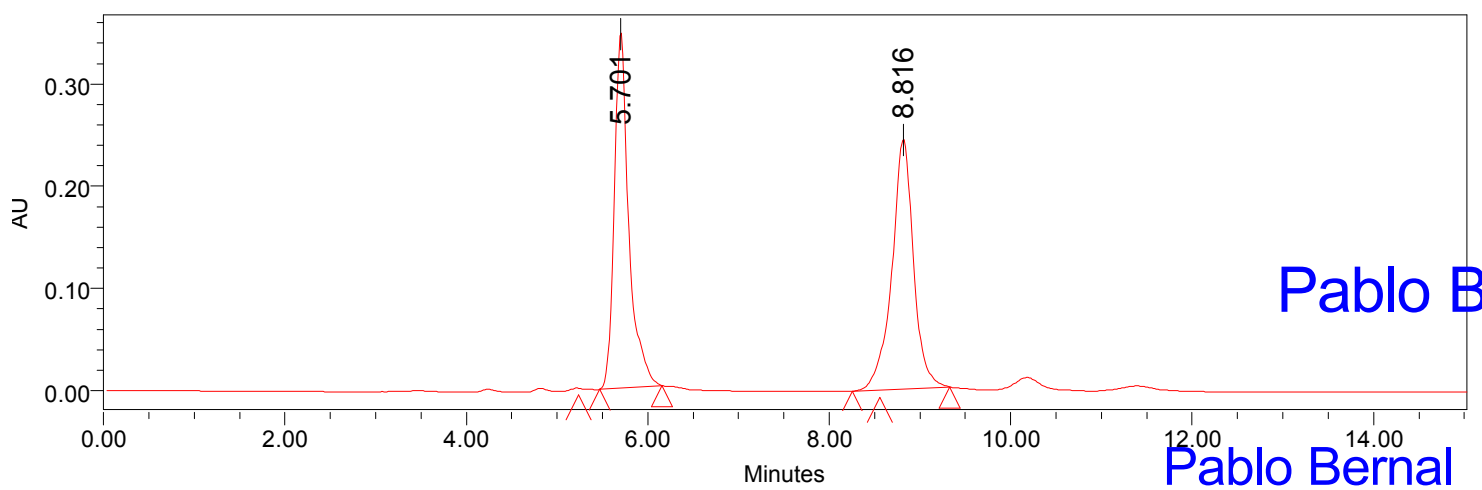

Processed Channel: PDA $220.0 \mathrm{~nm}$

\begin{tabular}{|r|l|r|c|r|r|}
\hline & Processed Channel & $\begin{array}{r}\text { Retention } \\
\text { Time }(\min )\end{array}$ & Area & \% Area & Height \\
\hline 1 & PDA 220.0 nm & 5.701 & 3886364 & 49.09 & 348457 \\
\hline 2 & PDA 220.0 nm & 8.816 & 4030764 & 50.91 & 244353 \\
\hline
\end{tabular}

Enantioriched sample of $\left(S_{a}, R\right)-7 \mathrm{c}$ :

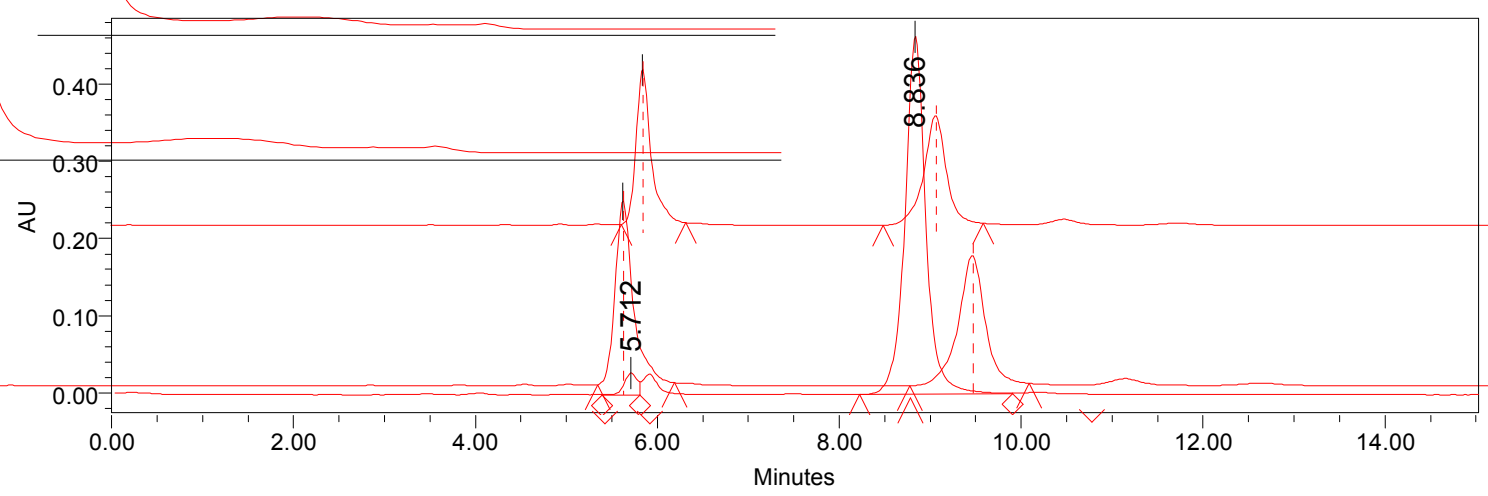

\section{Processed Channel: PDA $220.0 \mathrm{~nm}$}

\begin{tabular}{|r|l|r|r|r|r|}
\hline & Processed Channel & $\begin{array}{r}\text { Retention } \\
\text { Time }(\mathrm{min})\end{array}$ & Area & \% Area & Height \\
\hline 1 & PDA $220.0 \mathrm{~nm}$ & 5.712 & 276127 & 3.58 & 28469 \\
\hline 2 & PDA $220.0 \mathrm{~nm}$ & 8.836 & 7439888 & 96.42 & 464073 \\
\hline
\end{tabular}


${ }^{1} \mathrm{H}$ NMR $\left(400 \mathrm{MHz}, \mathrm{CDCl}_{3}\right)$ of $\left(S_{a}, R\right)-\mathbf{7 d}$ :

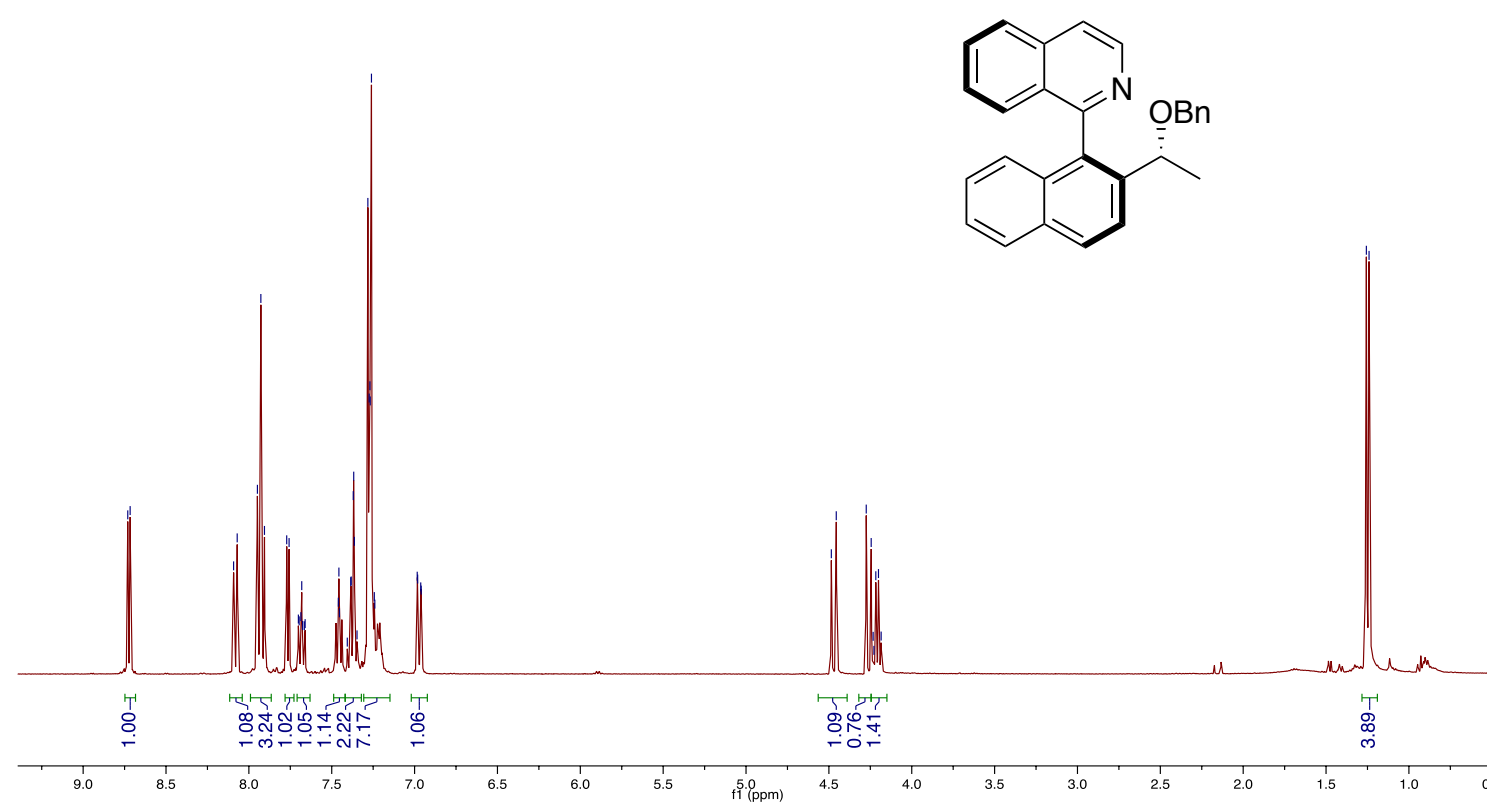

${ }^{13} \mathrm{C}$ NMR $\left(100 \mathrm{MHz}, \mathrm{CDCl}_{3}\right)$ of $\left(S_{a}, R\right)-7 \mathbf{d}$ :

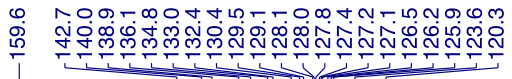
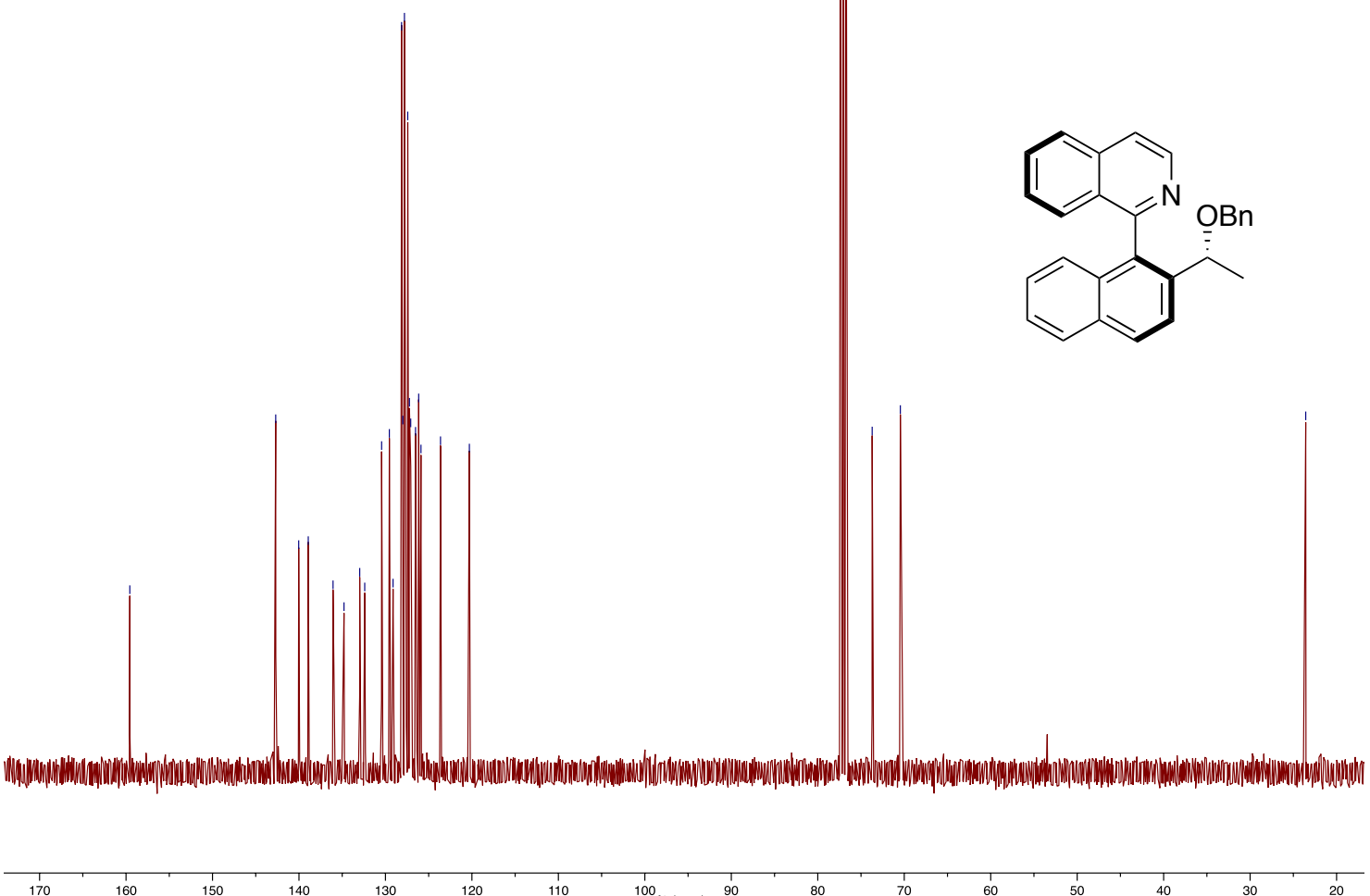

${ }_{100}{ }_{\mathrm{f1}(\mathrm{ppm})} 90$ 
Racemic sample of $( \pm)-7 \mathbf{d}$ : OD column, $n$-hexane:isopropanol 99:1, F= $1 \mathrm{~mL} / \mathrm{min}, \mathrm{T}=$ $30^{\circ} \mathrm{C}$.

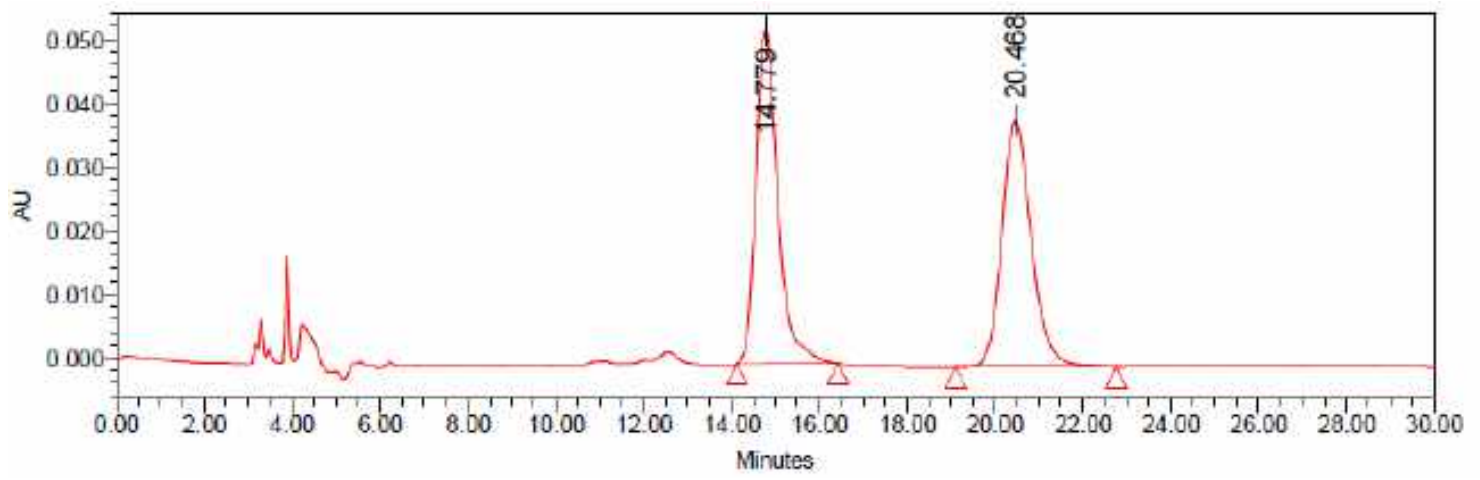

Processed Channel: PDA $220.0 \mathrm{~nm}$
\begin{tabular}{|l|l|r|r|r|r|}
\hline & Processed Channel & $\begin{array}{c}\text { Retention } \\
\text { Time }(\mathrm{min})\end{array}$ & Area & $\%$ Area & Height \\
\hline 1 & PDA $220.0 \mathrm{~nm}$ & 14.779 & 1816573 & 50.27 & 52381 \\
\hline 2 & PDA $220.0 \mathrm{~nm}$ & 20.468 & 1796825 & 49.73 & 38791 \\
\hline
\end{tabular}

Enantioriched sample of $\left(S_{a}, R\right)-7 \mathbf{d}$ :

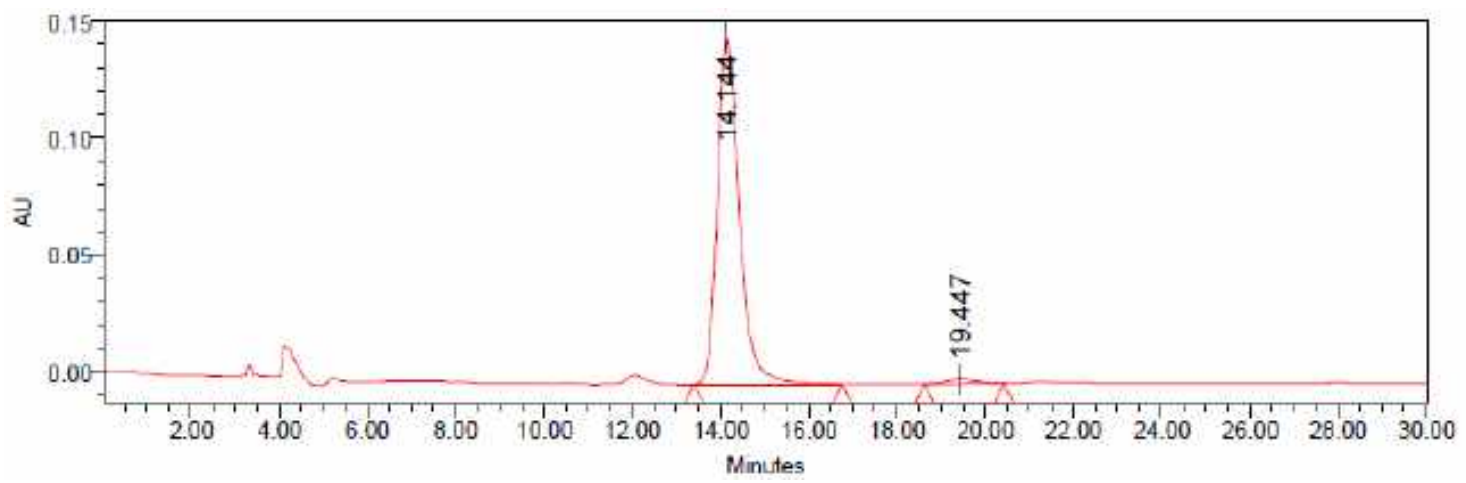

Processed Channel: PDA $220.0 \mathrm{~nm}$

\begin{tabular}{|r|l|r|r|r|r|}
\hline & Processed Channel & $\begin{array}{r}\text { Retention } \\
\text { Time (min) }\end{array}$ & Area & $\%$ Area & Height \\
\hline 1 & PDA 220.0 nm & 14.144 & 5089810 & 98.43 & 148080 \\
\hline 2 & PDA 220.0 nm & 19.447 & 81105 & 1.57 & 1841 \\
\hline
\end{tabular}


${ }^{1} \mathrm{H}$ NMR $\left(400 \mathrm{MHz}, \mathrm{CDCl}_{3}\right)$ of $\left(S_{a}, R\right)-7 \mathbf{e}:$

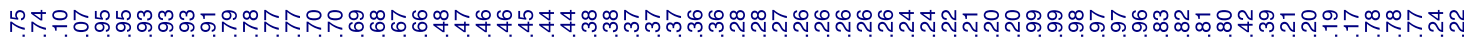
o

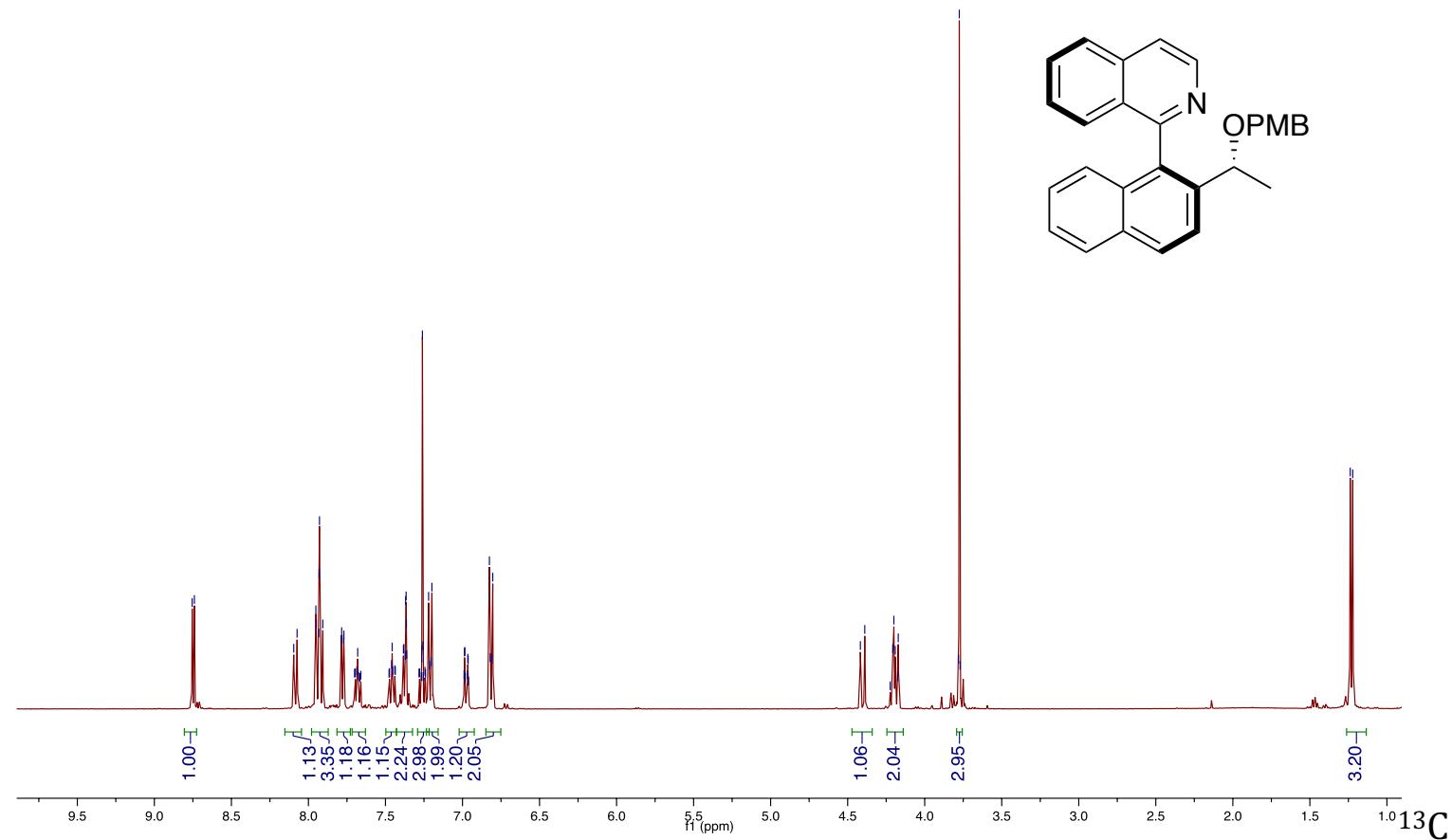

${ }^{13} \mathrm{C}-\mathrm{NMR}\left(100 \mathrm{MHz}, \mathrm{CDCl}_{3}\right)$ of $\left(S_{a}, R\right)-7 \mathbf{e}$ :

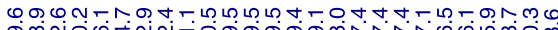

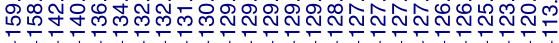
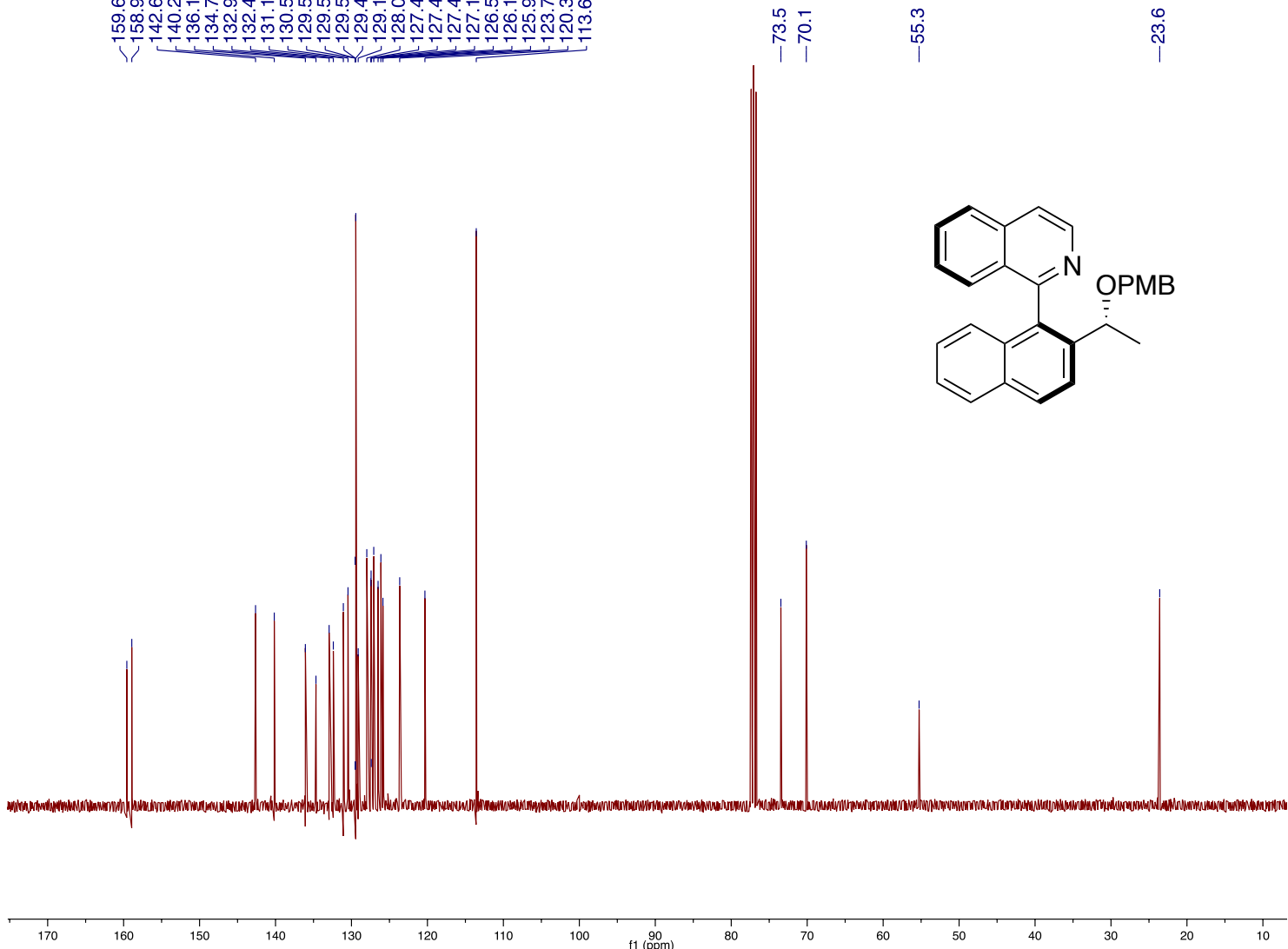
Racemic sample of $( \pm)$-7e: OD column, $n$-hexane:isopropanol 95:5, F= $1 \mathrm{~mL} / \mathrm{min}, \mathrm{T}=$ $30^{\circ} \mathrm{C}$

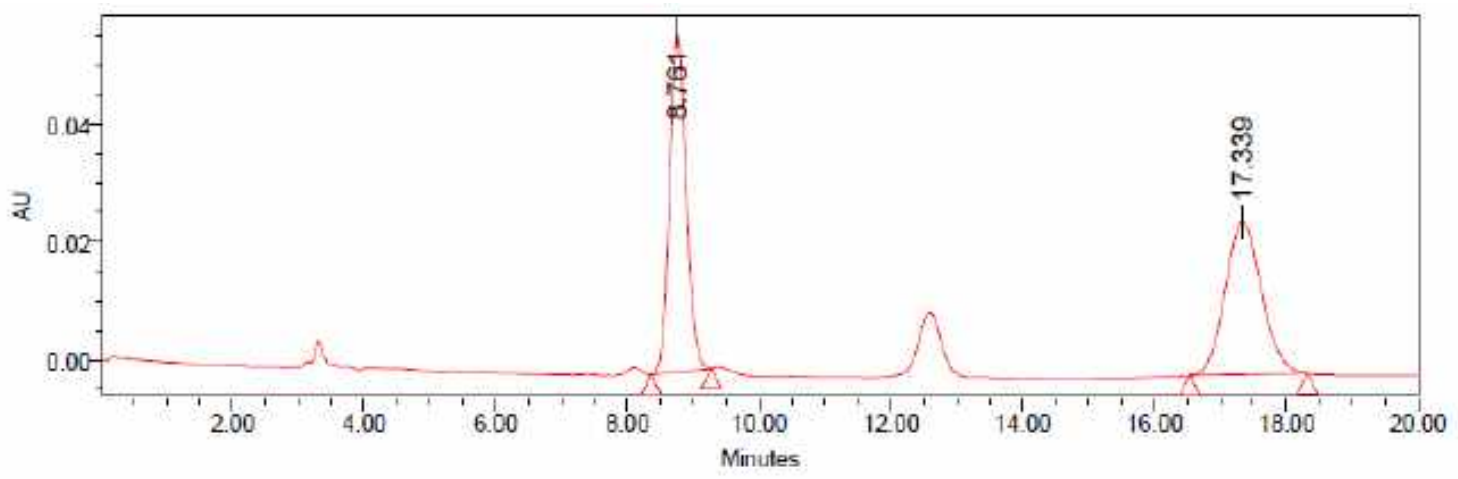

Processed Channel: PDA 220.0 nm
\begin{tabular}{|l|r|r|r|r|r|}
\hline & Processed Channel & $\begin{array}{l}\text { Retention } \\
\text { Time (min) }\end{array}$ & Area & $\%$ Area & Height \\
\hline 1 & PDA 220.0 nm & 8.761 & 1006856 & 50.24 & 57795 \\
\hline 2 & PDA 220.0 nm & 17.339 & 997096 & 49.76 & 26003 \\
\hline
\end{tabular}

Enantioriched sample of $\left(S_{a}, R\right)$-7e:

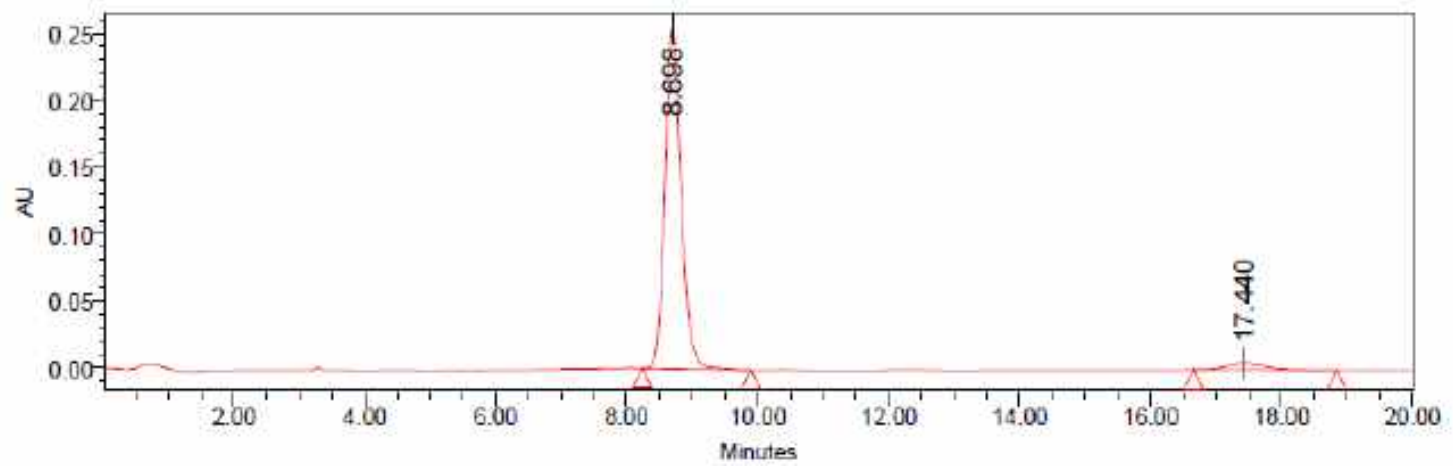

Processed Channel: PDA 220.0 nm
\begin{tabular}{|l|l|r|r|r|r|}
\hline & Processed Channel & $\begin{array}{r}\text { Retention } \\
\text { Time (min) }\end{array}$ & Area & $\%$ Area & Height \\
\hline 1 & PDA $220.0 \mathrm{~nm}$ & 8.698 & 4411422 & 95.31 & 253717 \\
\hline 2 & PDA $220.0 \mathrm{~nm}$ & 17.440 & 216966 & 4.69 & 5564 \\
\hline
\end{tabular}


${ }^{1} \mathrm{H}$ NMR $\left(400 \mathrm{MHz}, \mathrm{CDCl}_{3}\right)$ of $\left(S_{a}, R\right)-\mathbf{7 f}$ :

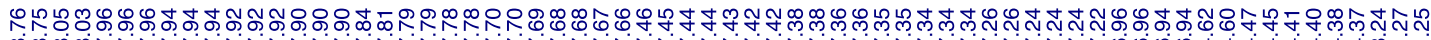
o

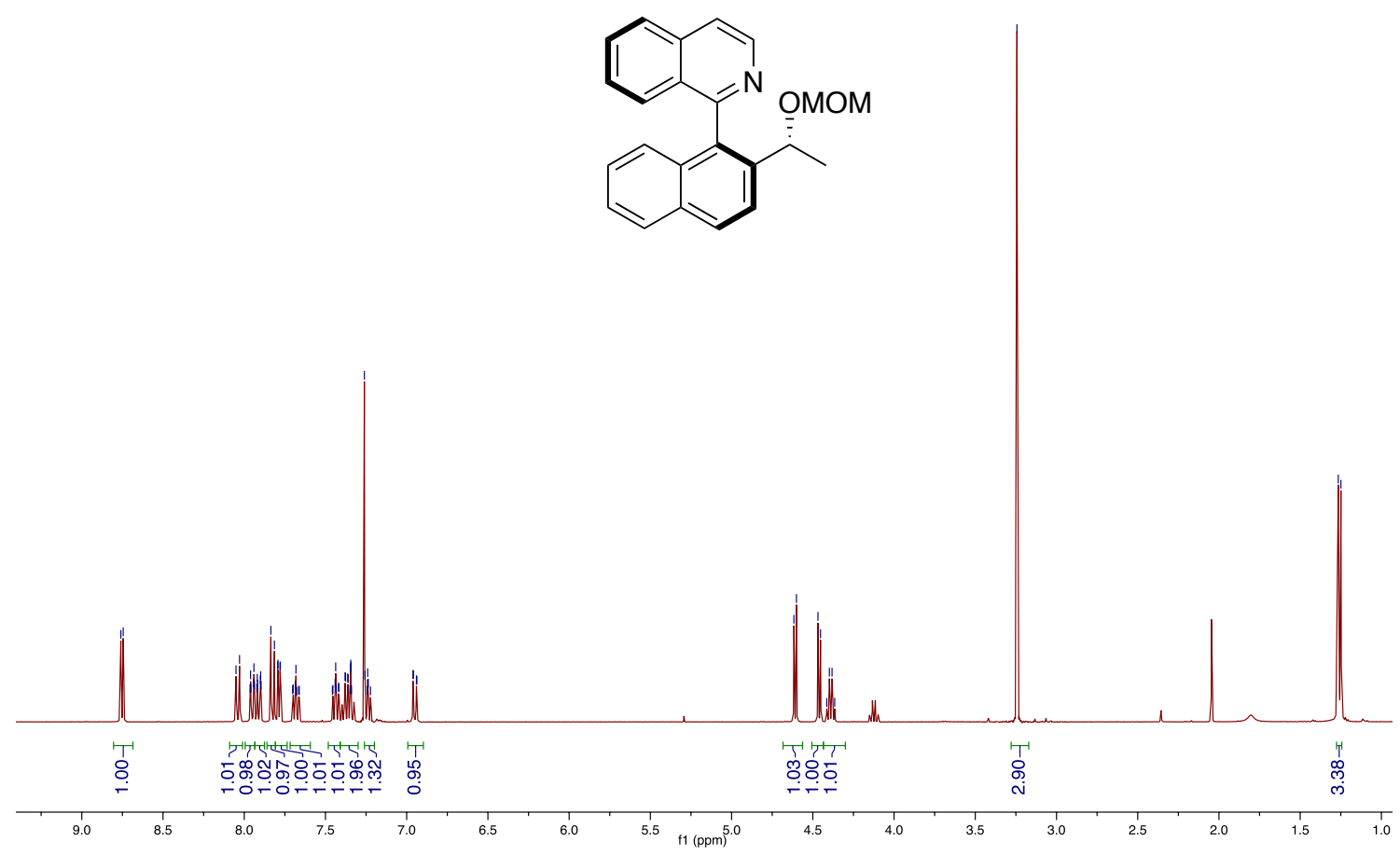

${ }^{13} \mathrm{C}$ NMR $\left(100 \mathrm{MHz}, \mathrm{CDCl}_{3}\right)$ of $\left(S_{a}, R\right)-7 \mathbf{f}$ :

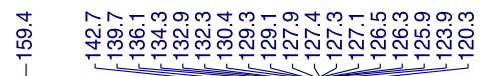

奋

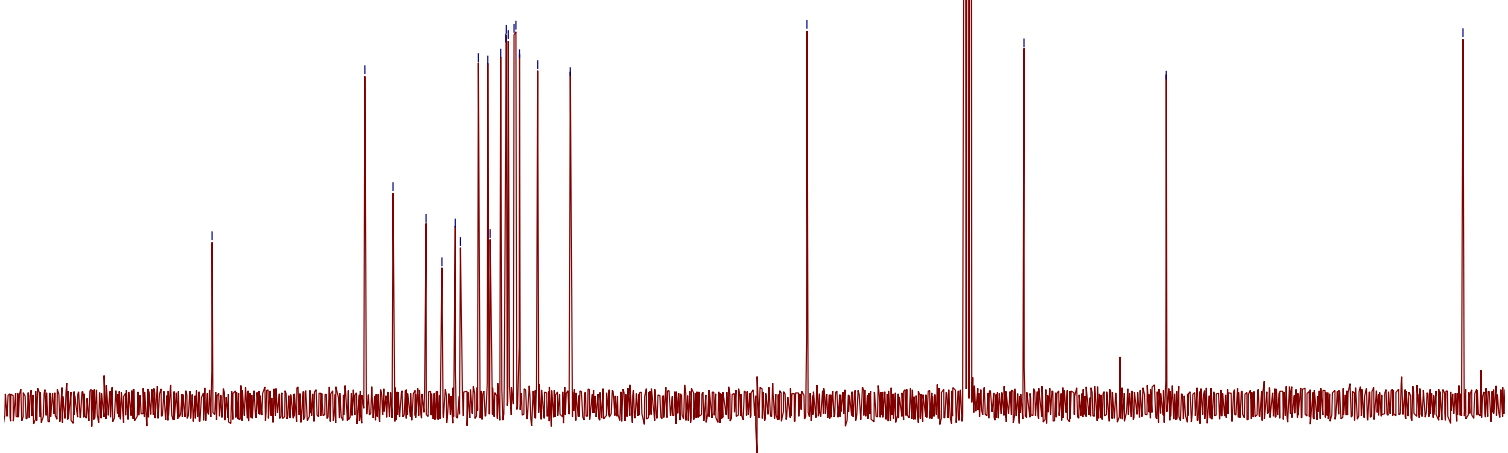

150

130

120

$110 \cdot \underset{f 1}{100}(\mathrm{ppm})$<smiles>COC(C)c1ccc2ccccc2c1-c1nccc2ccccc12</smiles>

1. 
Racemic sample of $( \pm$ )-7f: IA column, $n$-hexane:isopropanol 95:5, F= $1 \mathrm{~mL} / \mathrm{min}, \mathrm{T}=$ $30^{\circ} \mathrm{C}$

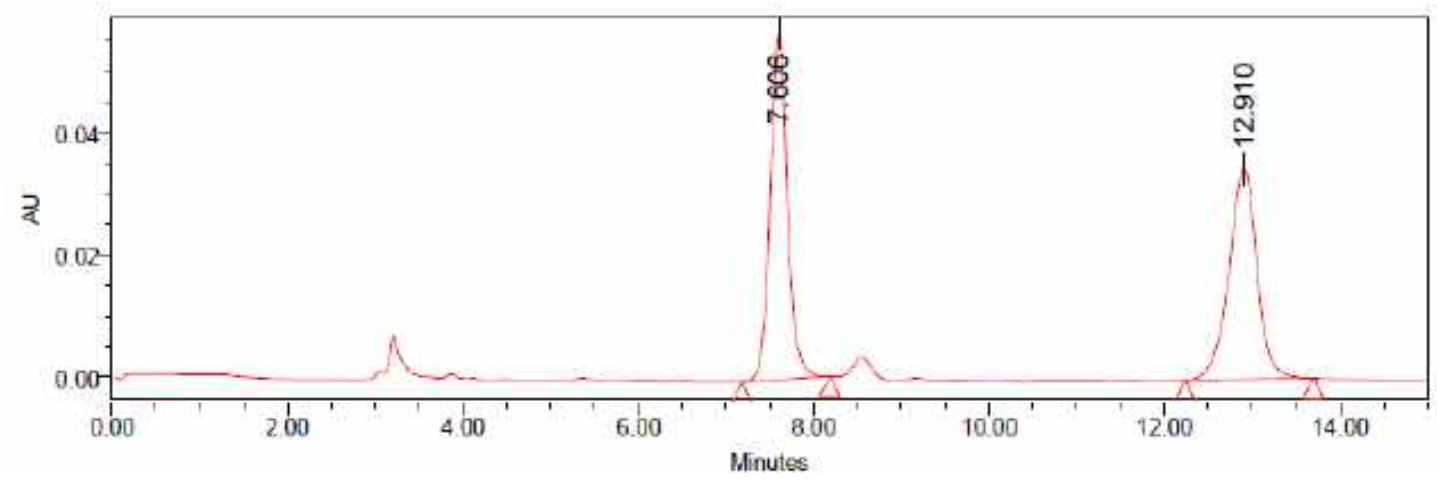

Processed Channel: PDA $220.0 \mathrm{~nm}$
\begin{tabular}{|l|l|r|r|r|r|}
\hline & Processed Channel & $\begin{array}{l}\text { Retention } \\
\text { Time (min) }\end{array}$ & Area & $\%$ Area & Height \\
\hline 1 & PDA $220.0 \mathrm{~nm}$ & 7.606 & 766991 & 49.98 & 56401 \\
\hline 2 & PDA $220.0 \mathrm{~nm}$ & 12.910 & 767576 & 50.02 & 34532 \\
\hline
\end{tabular}

Enantioriched sample of $\left(S_{a}, R\right)-7 \mathbf{f}$ :

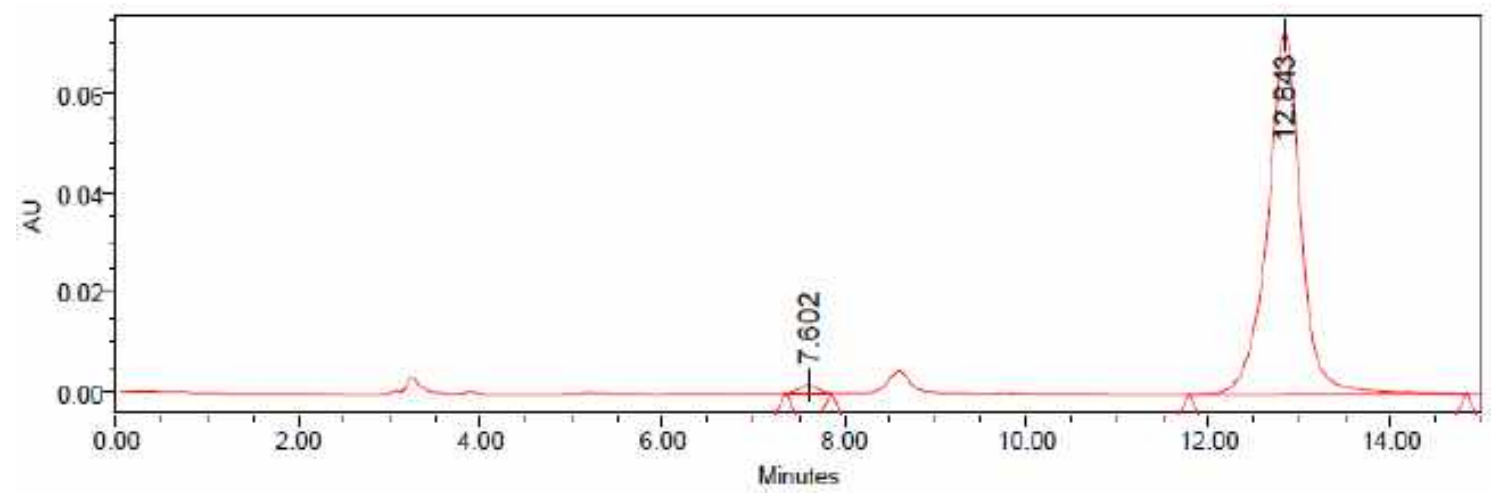

Processed Channel: PDA $220.0 \mathrm{~nm}$

\begin{tabular}{|l|l|r|r|r|r|}
\hline & Processed Channel & $\begin{array}{r}\text { Retention } \\
\text { Time (min) }\end{array}$ & Area & \% Area & Height \\
\hline 1 & PDA 220.0 nm & 7.602 & 21951 & 1.18 & 1655 \\
\hline 2 & PDA 220.0 nm & 12.843 & 1831326 & 98.82 & 72405 \\
\hline
\end{tabular}


${ }^{1} \mathrm{H} \mathrm{NMR}\left(400 \mathrm{MHz}, \mathrm{CDCl}_{3}\right)$ of the minor diastereomer of $\mathbf{7 g}$ :

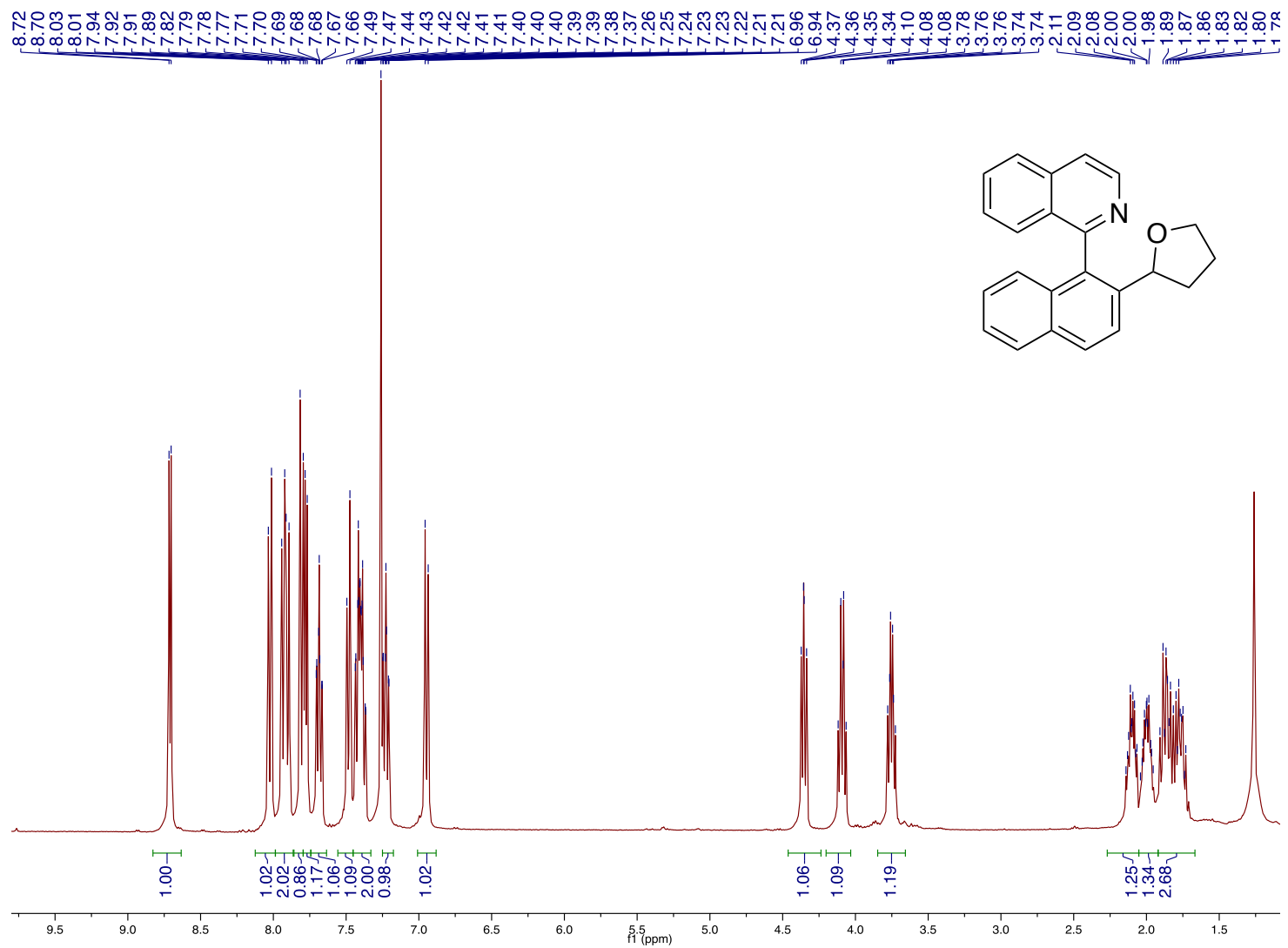

${ }^{13} \mathrm{C}$ NMR $\left(100 \mathrm{MHz}, \mathrm{CDCl}_{3}\right.$ ) of the minor diastereomer of $\mathbf{7 g}$ :

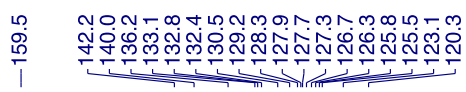

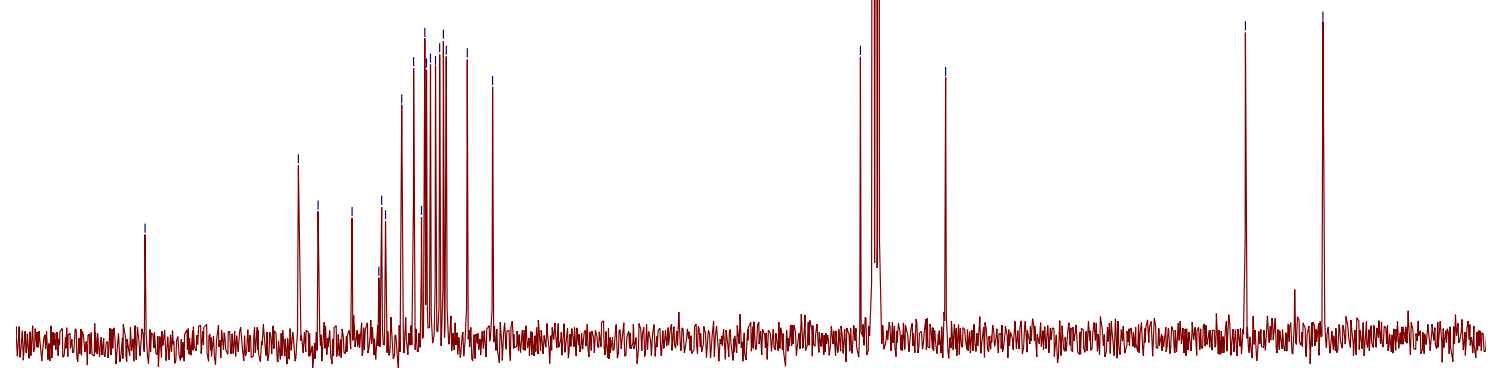

170

$120 \quad 110$

$100 \quad \mathrm{f1}\left(\begin{array}{c}90 \\ \mathrm{pm})\end{array}\right.$ 
Racemic sample $( \pm)$-minor diastereomer of $7 \mathrm{~g}$ : IB column, $n$-hexane:isopropanol 95:5, $\mathrm{F}=1 \mathrm{~mL} / \mathrm{min}, \mathrm{T}=30^{\circ} \mathrm{C}$.

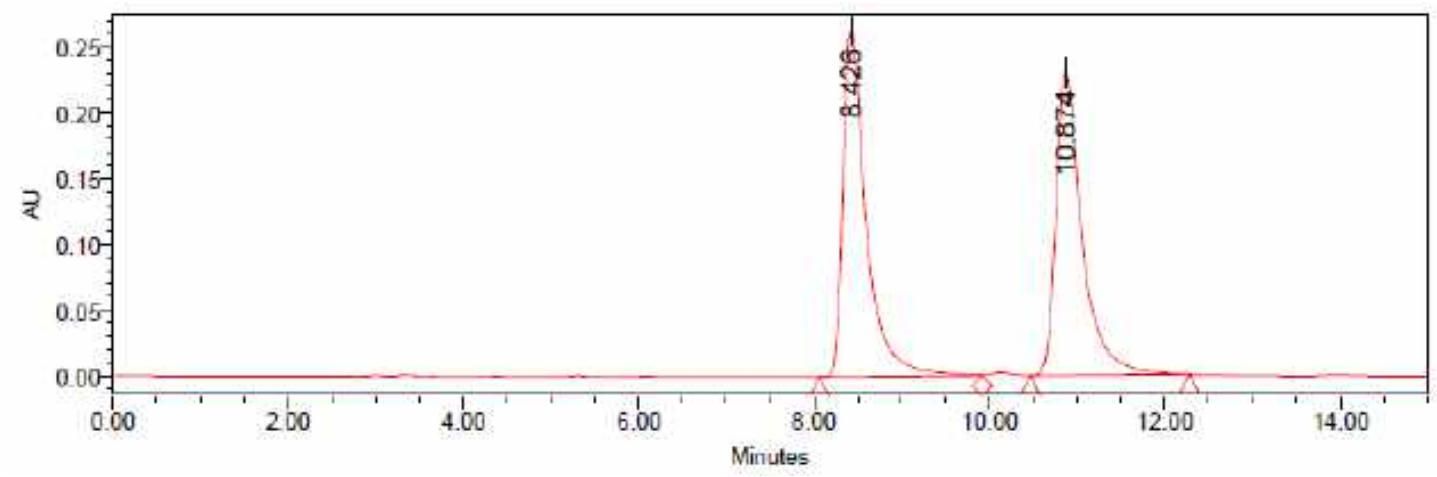

Processed Channel: PDA $225.0 \mathrm{~nm}$

\begin{tabular}{|l|l|r|c|r|r|}
\hline & Processed Channel & $\begin{array}{r}\text { Retention } \\
\text { Time }(\mathrm{min})\end{array}$ & Area & $\%$ Area & Height \\
\hline 1 & PDA 225.0 nm & 8.426 & 4849454 & 50.34 & 264077 \\
\hline 2 & PDA 225.0 nm & 10.874 & 4784336 & 49.66 & 230724 \\
\hline
\end{tabular}

\section{Enantioriched sample minor diastereomer of $7 \mathrm{~g}$ :}

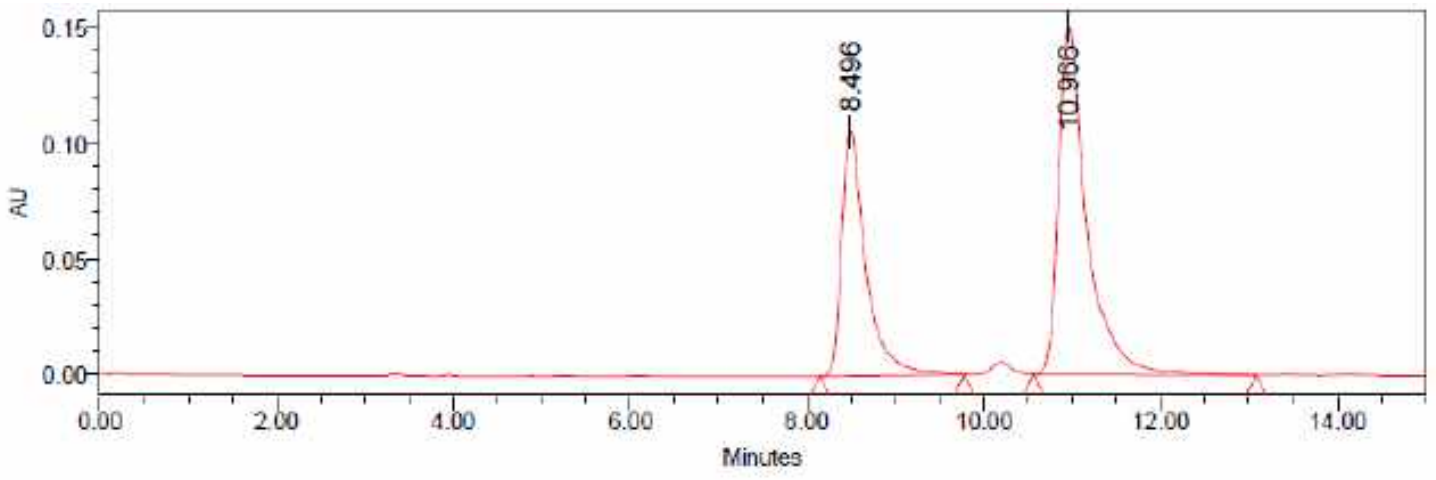

Processed Channel: PDA $225.0 \mathrm{~nm}$

\begin{tabular}{|l|l|r|r|r|r|}
\hline & Processed Channel & $\begin{array}{c}\text { Retention } \\
\text { Time }(\mathrm{min})\end{array}$ & Area & \% Area & Height \\
\hline 1 & PDA $225.0 \mathrm{~nm}$ & 8.496 & 2008577 & 37.61 & 105858 \\
\hline 2 & PDA $225.0 \mathrm{~nm}$ & 10.966 & 3331812 & 62.39 & 150520 \\
\hline
\end{tabular}


${ }^{1} \mathrm{H}$ NMR $\left(400 \mathrm{MHz}, \mathrm{CDCl}_{3}\right)$ of $\left(R_{a}, S\right)-\mathbf{7 g}$ :

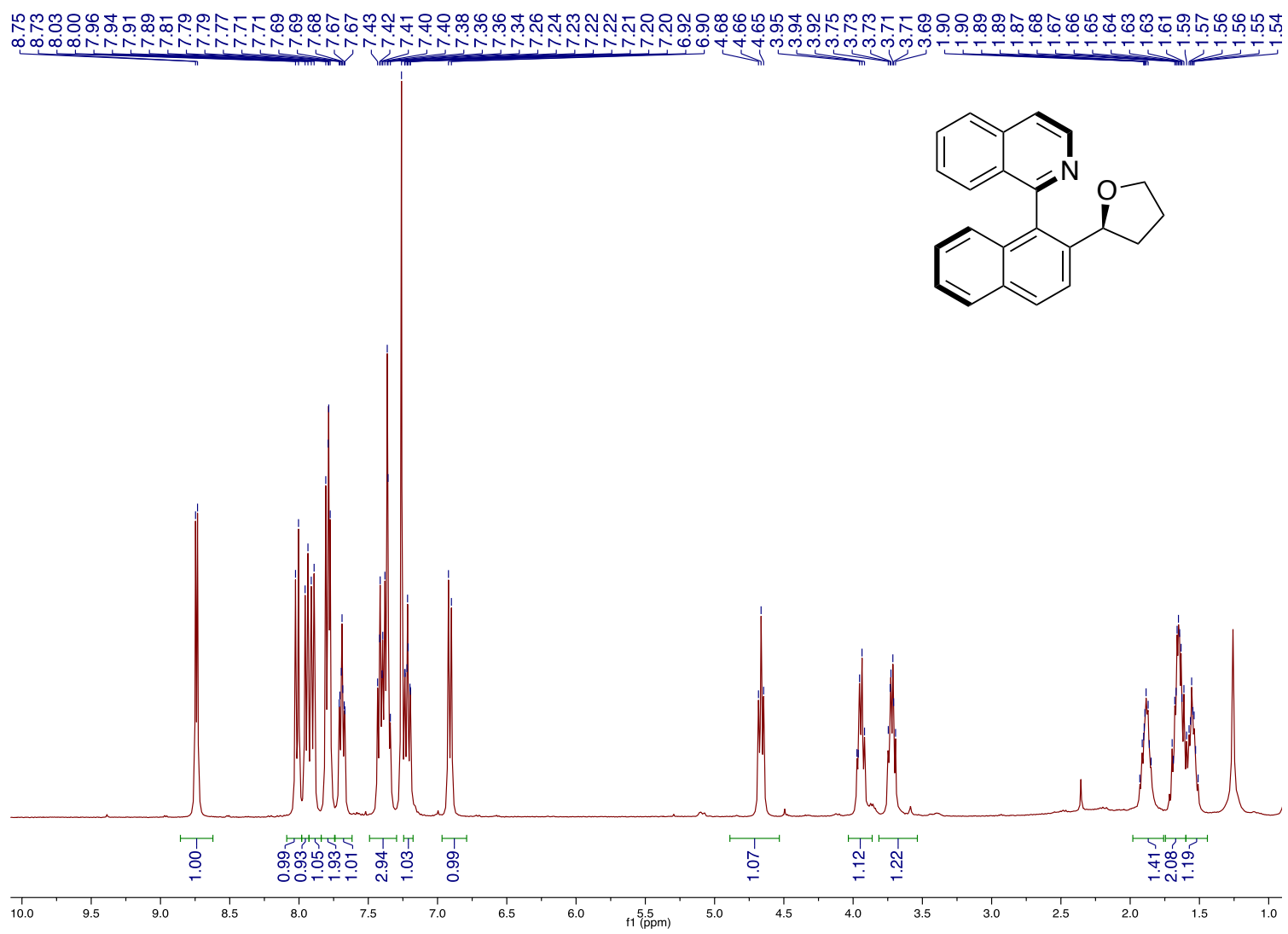

${ }^{13} \mathrm{C}$ NMR $\left(100 \mathrm{MHz}, \mathrm{CDCl}_{3}\right)$ of $\left(R_{a}, S\right)-\mathbf{7 g}$ :

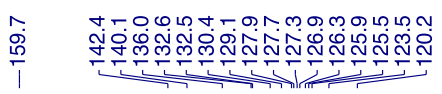

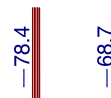

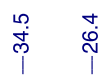<smiles>c1ccc2c(-c3ccc([C@H]4CCCO4)c4ccccc34)nccc2c1</smiles>

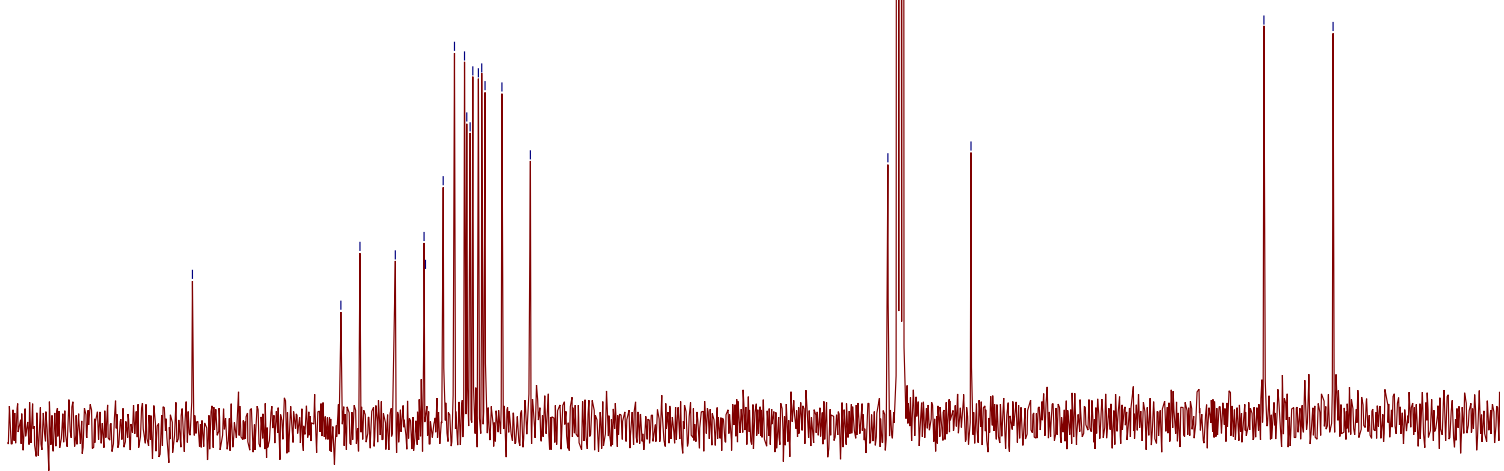

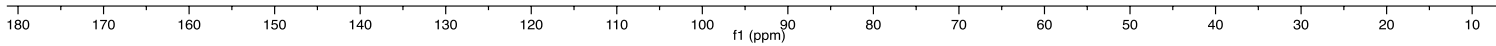


Racemic sample $( \pm)$-major diastereomer of $\mathbf{7 g}$ : IA column, $n$-hexane:isopropanol $90: 10, \mathrm{~F}=1 \mathrm{~mL} / \mathrm{min}, \mathrm{T}=30^{\circ} \mathrm{C}$.

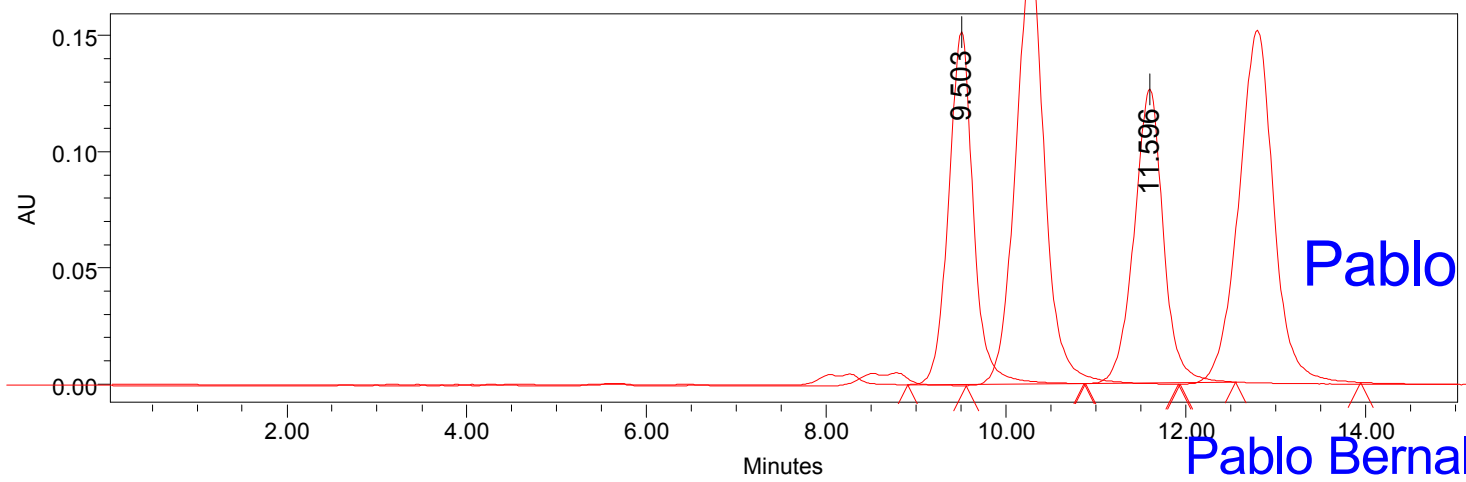

Processed Channel: PDA 229.1 nm
\begin{tabular}{|l|l|r|c|r|r|}
\hline & Processed Channel & $\begin{array}{c}\text { Retention } \\
\text { Time (min) }\end{array}$ & Area & $\%$ Area & Height \\
\hline 1 & PDA $229.1 \mathrm{~nm}$ & 9.503 & 2903657 & 50.52 & 151675 \\
\hline 2 & PDA $229.1 \mathrm{~nm}$ & 11.596 & 2843775 & 49.48 & 126356 \\
\hline
\end{tabular}

Enantioriched sample of $\left(R_{a}, S\right)-7 \mathbf{g}$ :

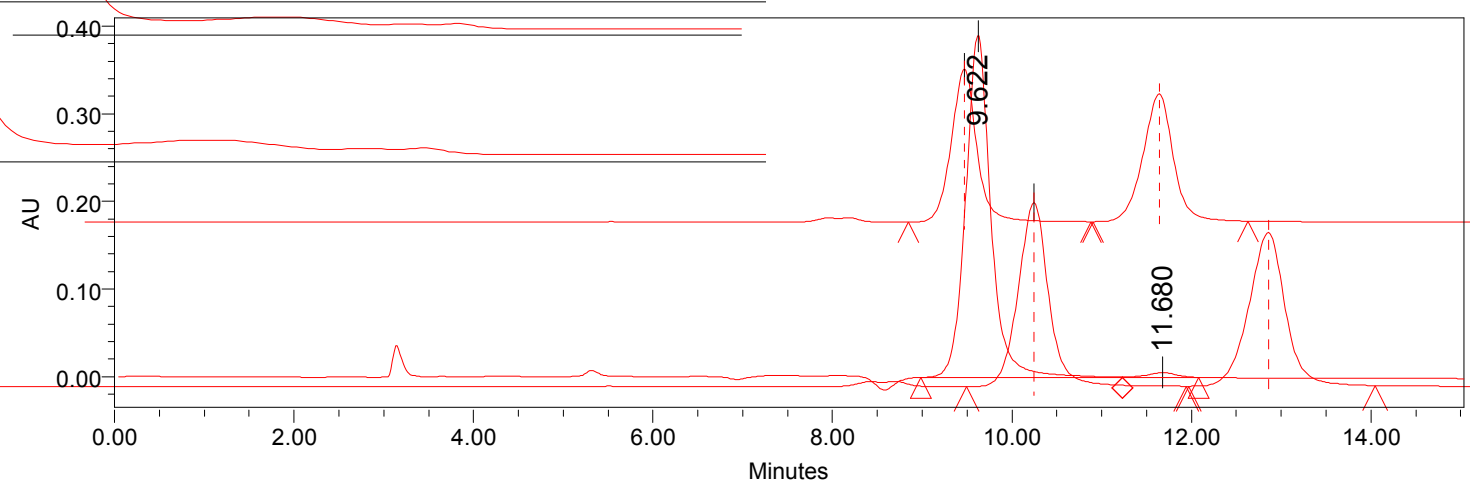

Processed Channel: PDA 225.1 nm

\begin{tabular}{|r|l|r|r|r|r|}
\hline & Processed Channel & $\begin{array}{r}\text { Retention } \\
\text { Time (min) }\end{array}$ & Area & \% Area & Height \\
\hline 1 & PDA 225.1 nm & 9.622 & 7655359 & 98.46 & 390840 \\
\hline 2 & PDA $225.1 \mathrm{~nm}$ & 11.680 & 119966 & 1.54 & 5461 \\
\hline
\end{tabular}


${ }^{1} \mathrm{H}$ NMR $\left(400 \mathrm{MHz}, \mathrm{CDCl}_{3}\right)$ of $\left(S_{a}, R\right)-\mathbf{8 a}$ :

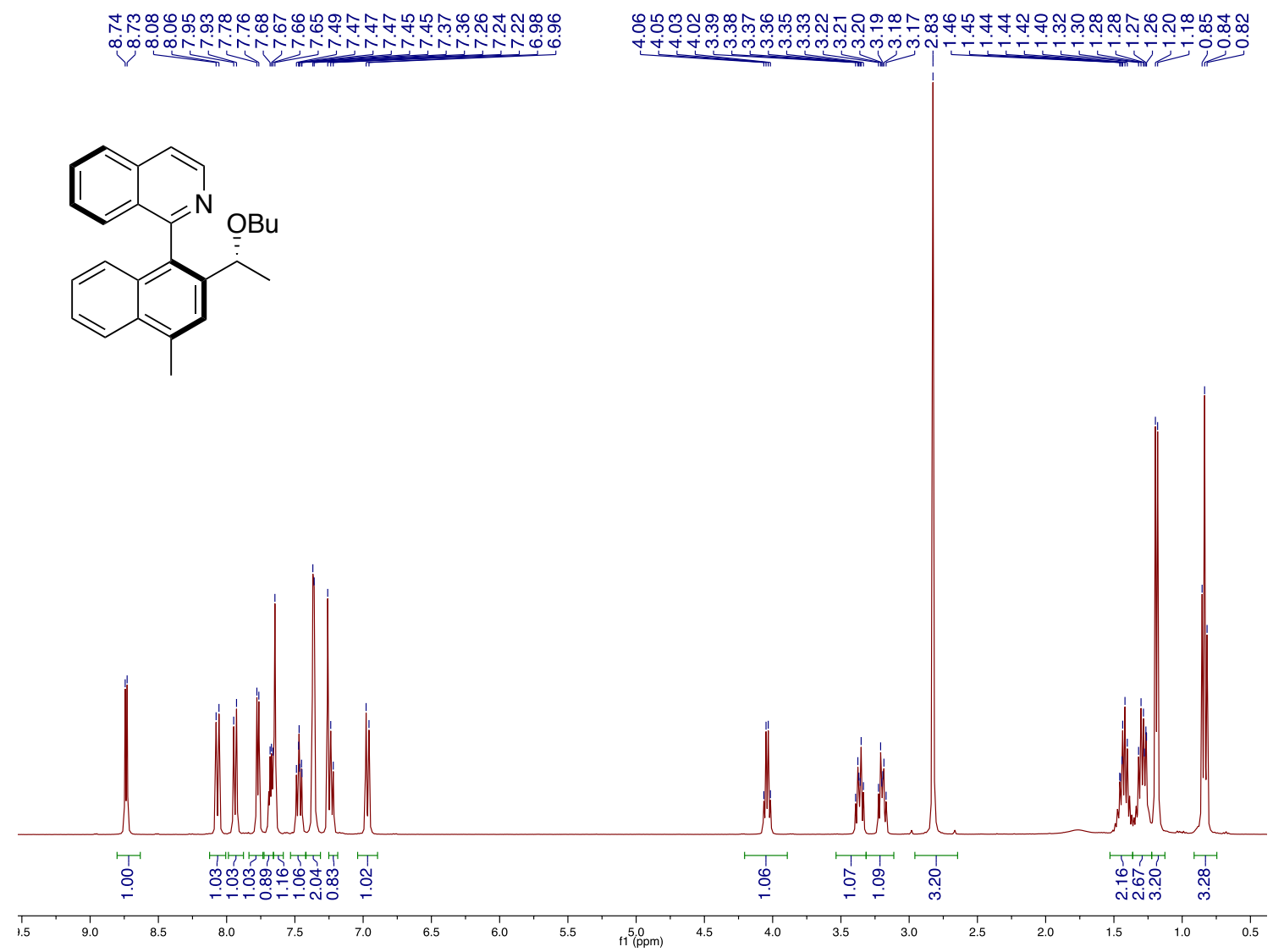

${ }^{13} \mathrm{C}$ NMR $\left(100 \mathrm{MHz}, \mathrm{CDCl}_{3}\right)$ of $\left(S_{a}, R\right)-\mathbf{8 a}$ :

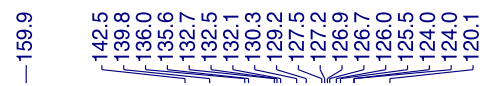

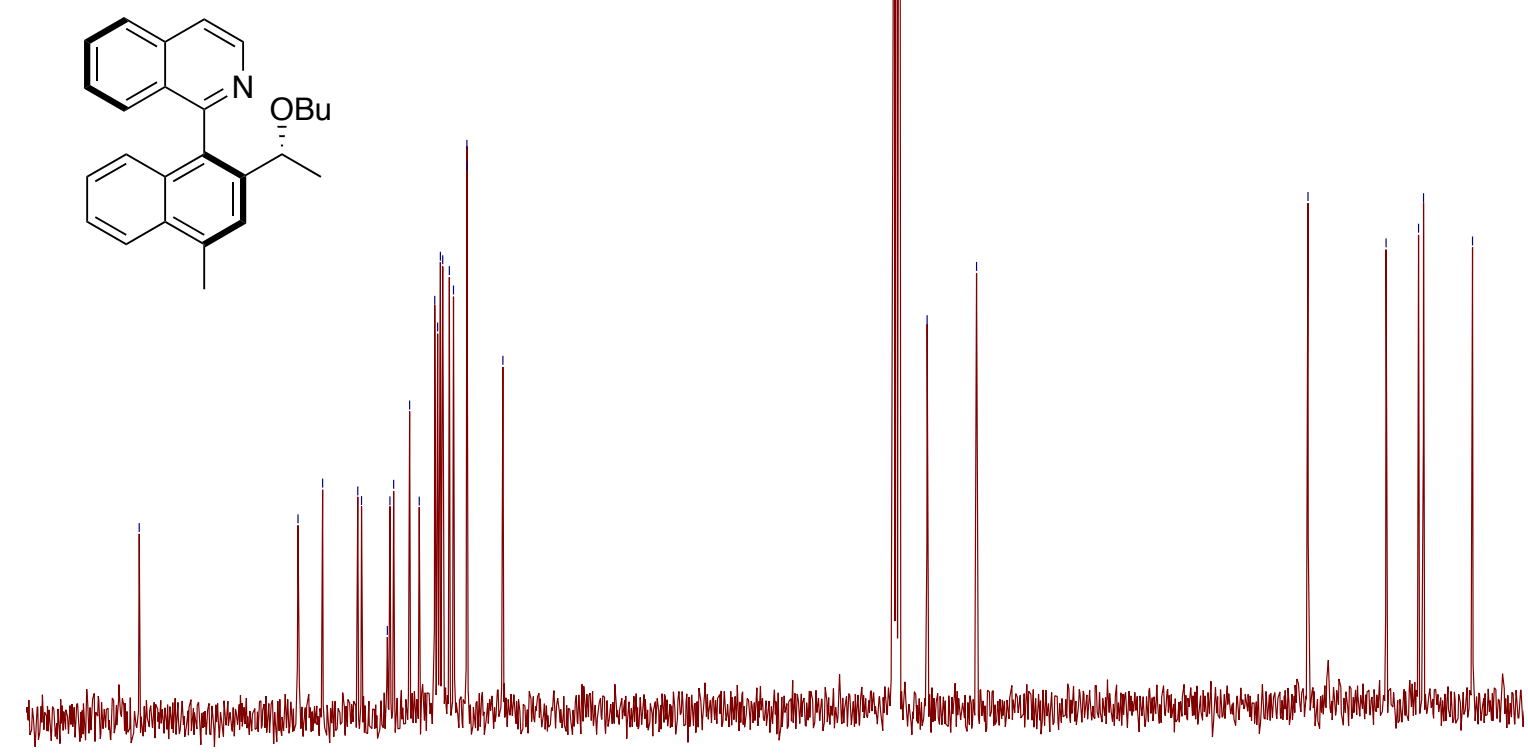

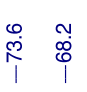

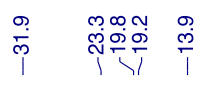

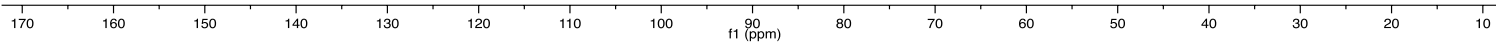


Racemic sample of $( \pm)$-8a: IA column, $n$-hexane:isopropanol 99:1, F= $1 \mathrm{~mL} / \mathrm{min}, \mathrm{T}=$ $30^{\circ} \mathrm{C}$.

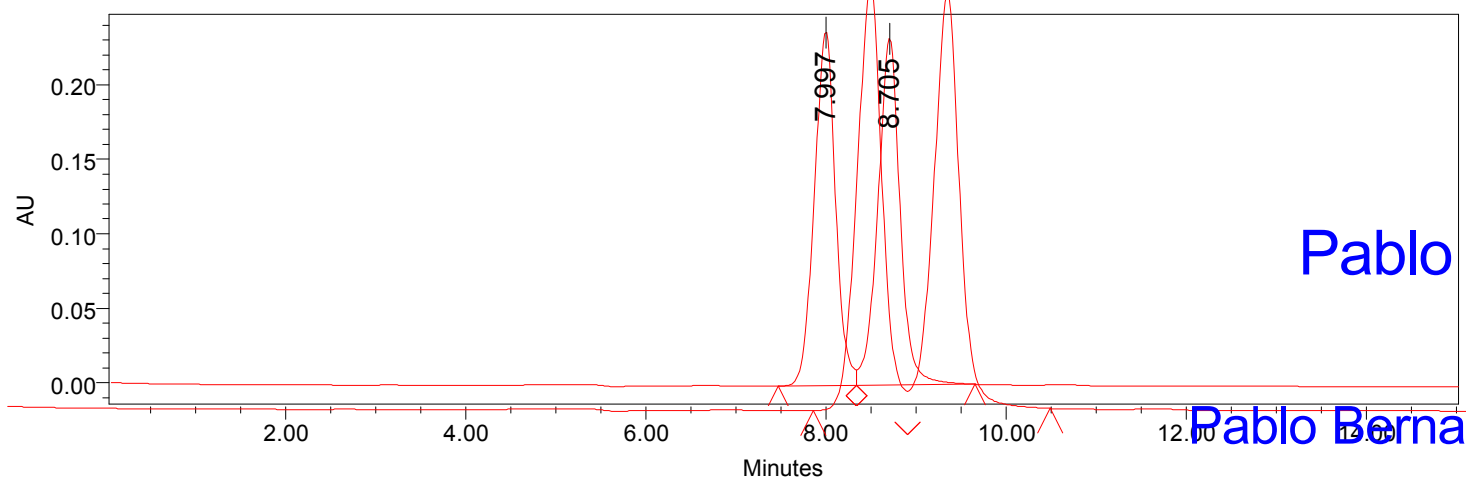

\section{Processed Channel: PDA $220.0 \mathrm{~nm}$}

\begin{tabular}{|r|l|r|c|r|r|}
\hline & Processed Channel & $\begin{array}{r}\text { Retention } \\
\text { Time }(\min )\end{array}$ & Area & \% Area & Height \\
\hline 1 & PDA 220.0 nm & 7.997 & 3879977 & 49.28 & 237606 \\
\hline 2 & PDA 220.0 nm & 8.705 & 3993297 & 50.72 & 232425 \\
\hline
\end{tabular}

Enantioriched sample of $\left(S_{a}, R\right)$-8a:

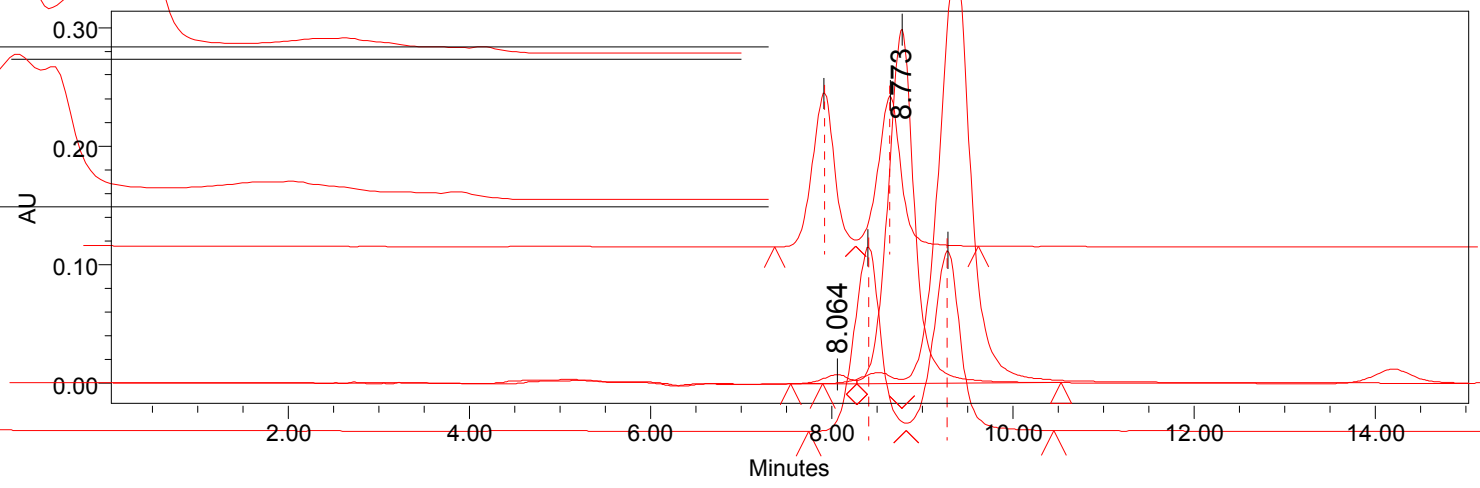

\section{Processed Channel: PDA $220.8 \mathrm{~nm}$}

\begin{tabular}{|r|l|r|r|r|r|}
\hline & Processed Channel & $\begin{array}{c}\text { Retention } \\
\text { Time (min) }\end{array}$ & Area & \% Area & Height \\
\hline 1 & PDA 220.8 nm & 8.064 & 150268 & 2.39 & 7664 \\
\hline 2 & PDA 220.8 nm & 8.773 & 6137962 & 97.61 & 299357 \\
\hline
\end{tabular}


${ }^{1} \mathrm{H}$ NMR $\left(400 \mathrm{MHz}, \mathrm{CDCl}_{3}\right)$ of $\left(S_{a}, R\right)-\mathbf{8 b}$ :

ハ下ロ

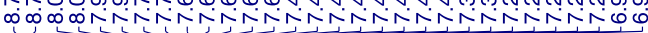

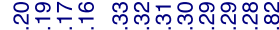

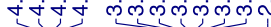

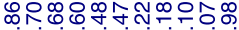

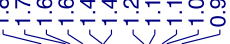

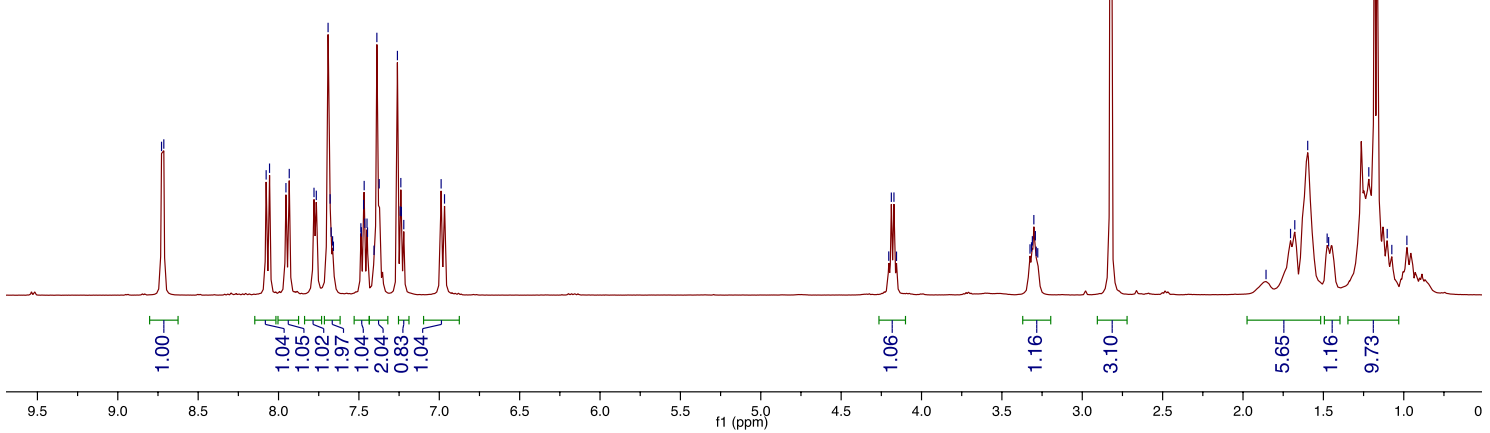

${ }^{13} \mathrm{C}$ NMR $\left(100 \mathrm{MHz}, \mathrm{CDCl}_{3}\right)$ of $\left(S_{a}, R\right)-\mathbf{8 b}$ :

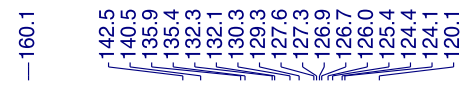<smiles>Cc1cc(C(C)OC(C)C)c(-c2nccc3ccccc23)c2ccccc12</smiles>
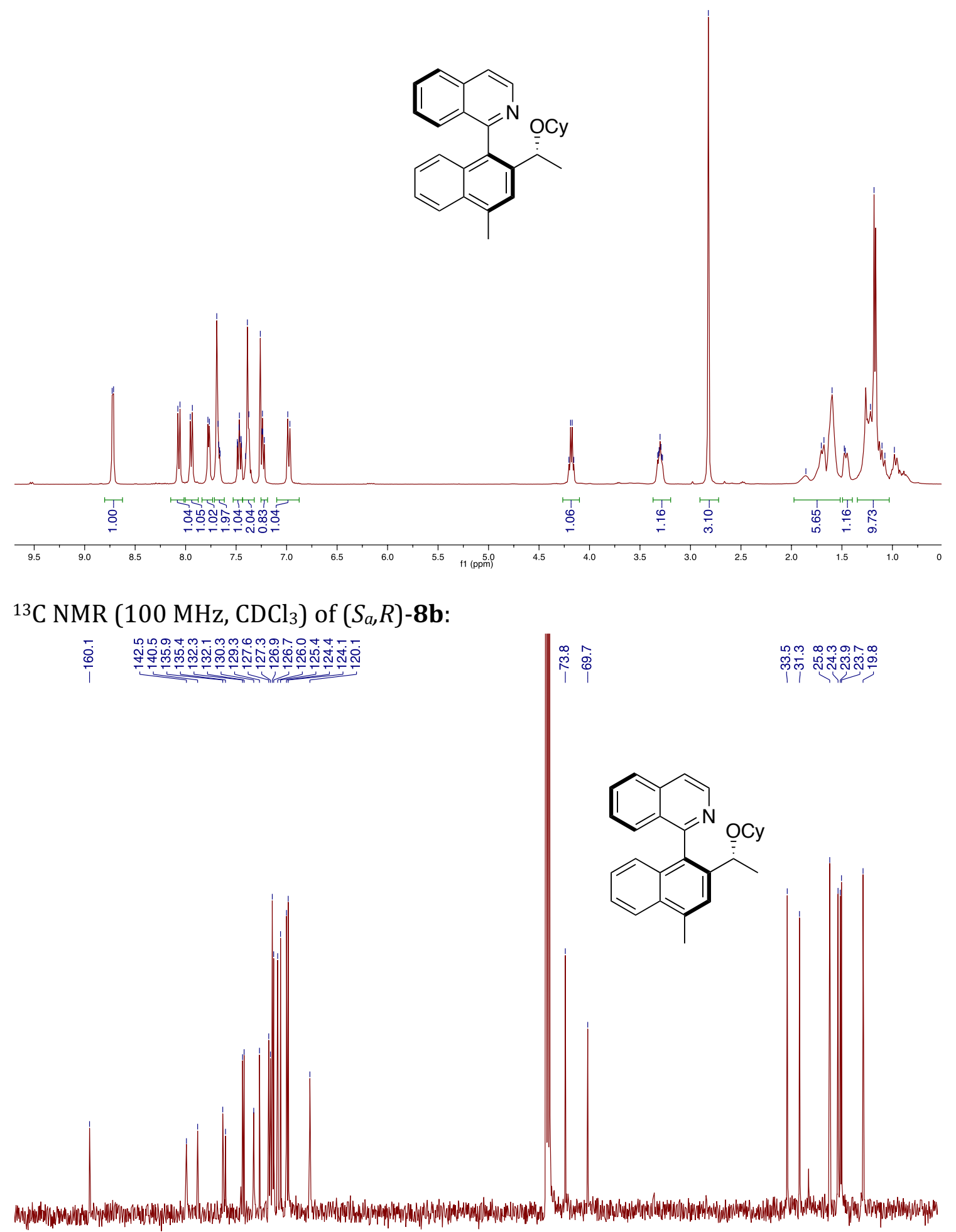

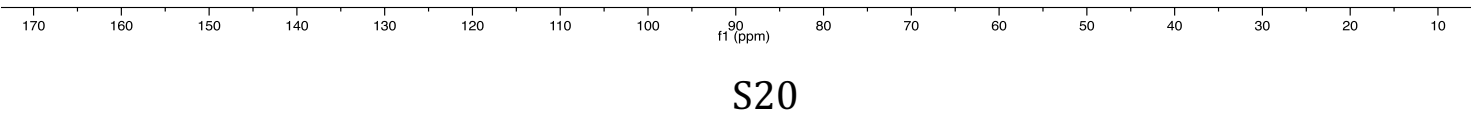


Racemic sample of $( \pm)$-8b: IA column, $n$-hexane:isopropanol 99:1, F= $1 \mathrm{~mL} / \mathrm{min}, \mathrm{T}=$ $30^{\circ} \mathrm{C}$.

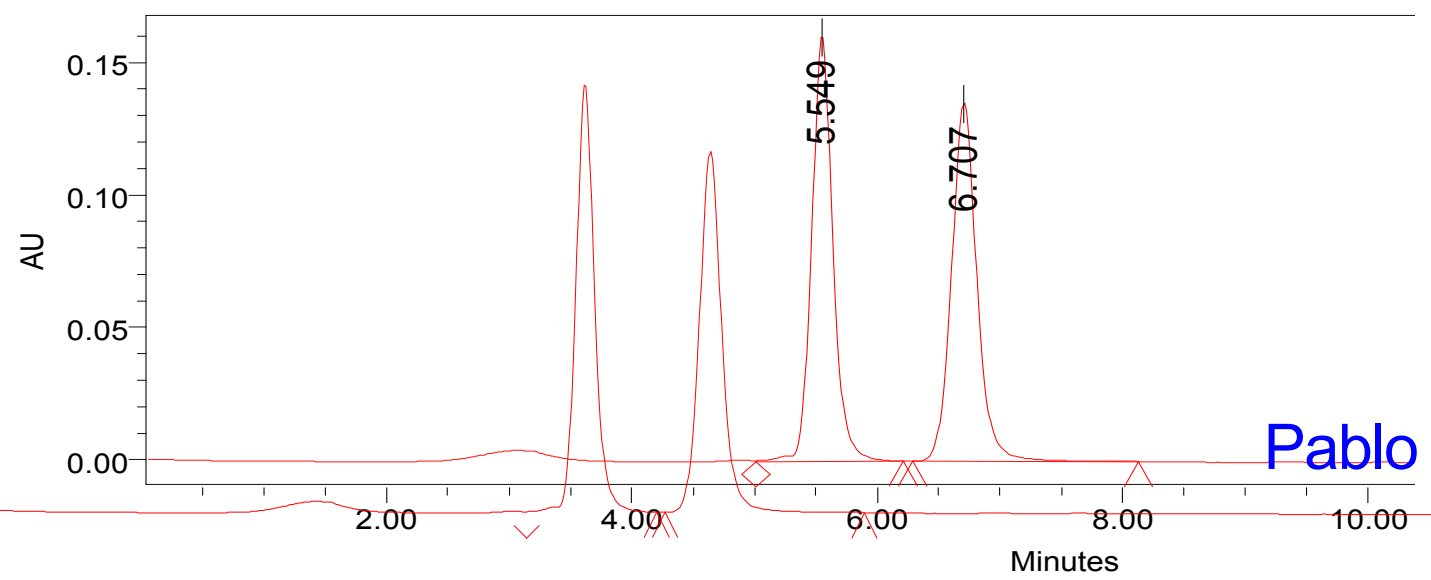

Processed Channel: PDA $219.0 \mathrm{~nm}$

\begin{tabular}{|l|l|r|r|r|r|}
\hline & Processed Channel & $\begin{array}{r}\text { Retention } \\
\text { Time }(\mathrm{min})\end{array}$ & Area & \% Area & Height \\
\hline 1 & PDA $219.0 \mathrm{~nm}$ & 5.549 & 1946526 & 49.97 & 161158 \\
\hline 2 & PDA $219.0 \mathrm{~nm}$ & 6.707 & 1948664 & 50.03 & 135360 \\
\hline
\end{tabular}

Enantioriched sample of $\left(S_{a}, R\right)-8 \mathbf{b}$ :

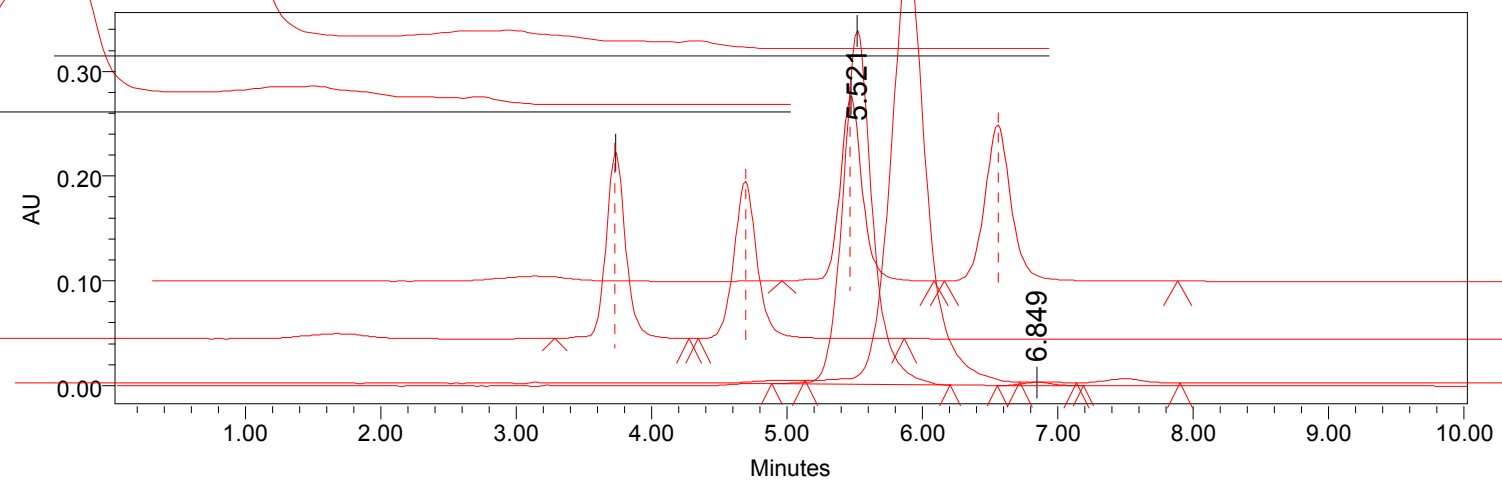

\section{Processed Channel: PDA 223.4 nm}

\begin{tabular}{|r|l|r|r|r|r|}
\hline & Processed Channel & $\begin{array}{r}\text { Retention } \\
\text { Time }(\min )\end{array}$ & Area & \% Area & Height \\
\hline 1 & PDA 223.4 nm & 5.521 & 5139579 & 99.07 & 337618 \\
\hline 2 & PDA 223.4 nm & 6.849 & 48443 & 0.93 & 3241 \\
\hline
\end{tabular}


${ }^{1} \mathrm{H}$ NMR $\left(400 \mathrm{MHz}, \mathrm{CDCl}_{3}\right)$ of $\left(S_{a}, R\right)-\mathbf{8 c}$ :

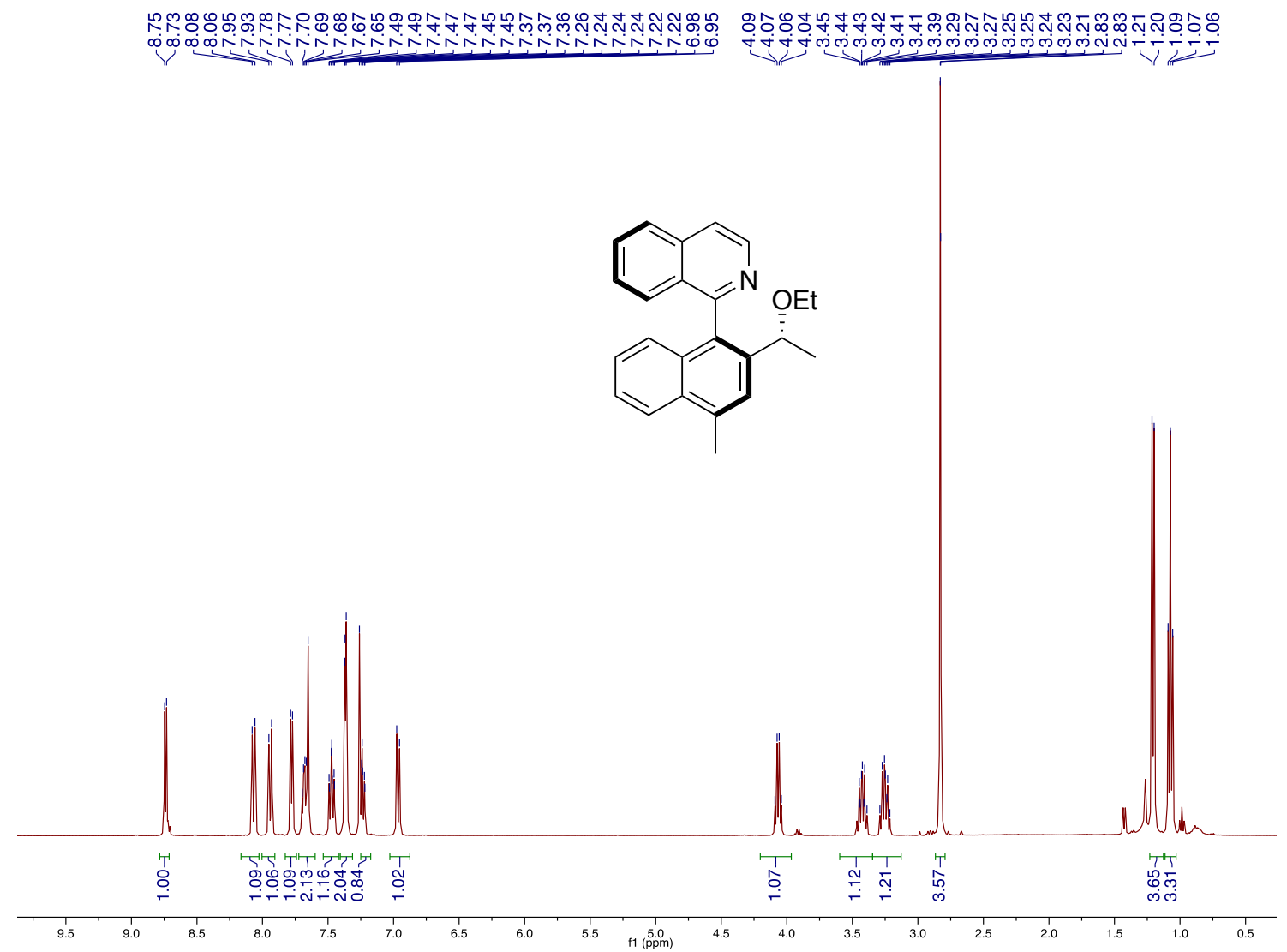

${ }^{13} \mathrm{C}$ NMR $\left(100 \mathrm{MHz}, \mathrm{CDCl}_{3}\right)$ of $\left(S_{a}, R\right)-\mathbf{8 c}$ :

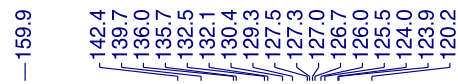

|ling

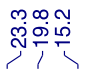<smiles>CCOc1cc(C)c2ccccc2c1-c1nccc2ccccc12</smiles>

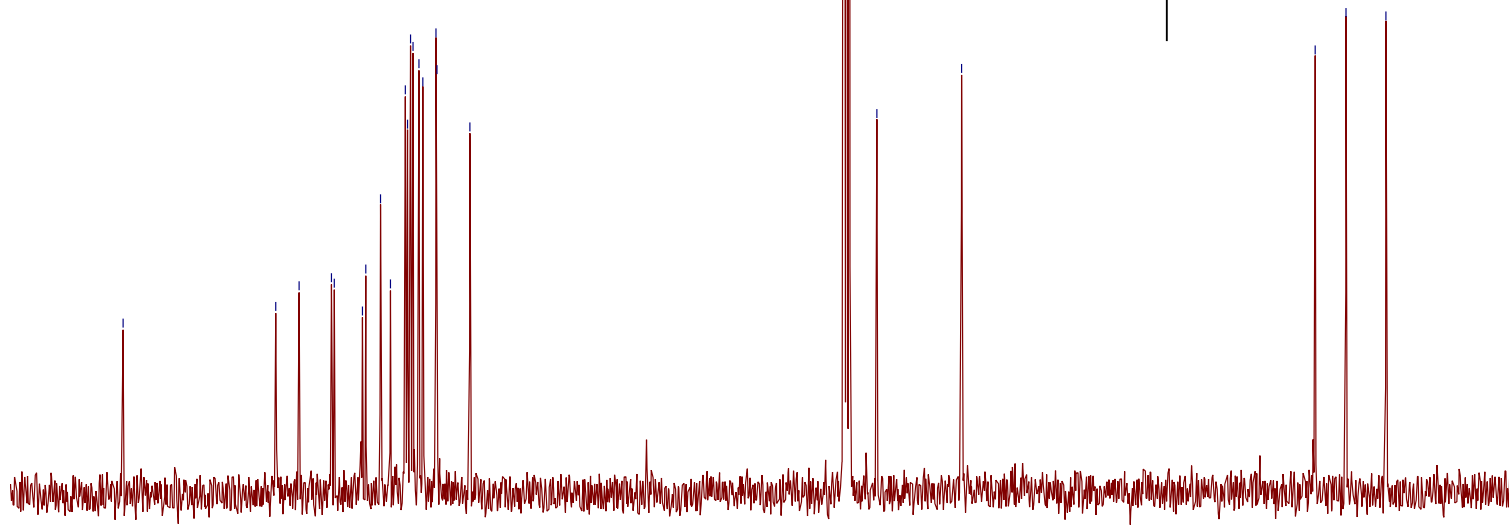


Racemic sample of $( \pm)$-8c: IA column, $n$-hexane:isopropanol 97:3, F= $1 \mathrm{~mL} / \mathrm{min}, \mathrm{T}=$ $30^{\circ} \mathrm{C}$.

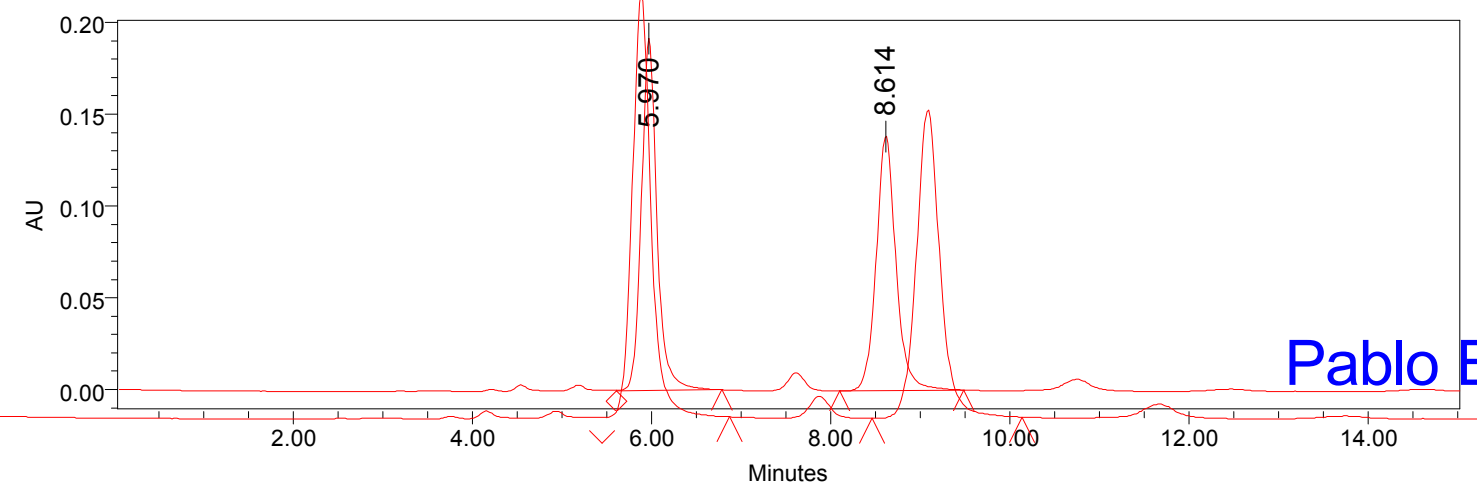

Processed Channel: PDA $220.0 \mathrm{~nm}$

\begin{tabular}{|l|l|r|c|r|r|}
\hline & Processed Channel & $\begin{array}{c}\text { Retention } \\
\text { Time (min) }\end{array}$ & Area & \% Area & Height \\
\hline 1 & PDA 220.0 nm & 5.970 & 2320059 & 52.03 & 191785 \\
\hline 2 & PDA 220.0 nm & 8.614 & 2138685 & 47.97 & 138859 \\
\hline
\end{tabular}

Enantioriched sample of $\left(S_{a}, R\right)-8 \mathrm{c}$ :

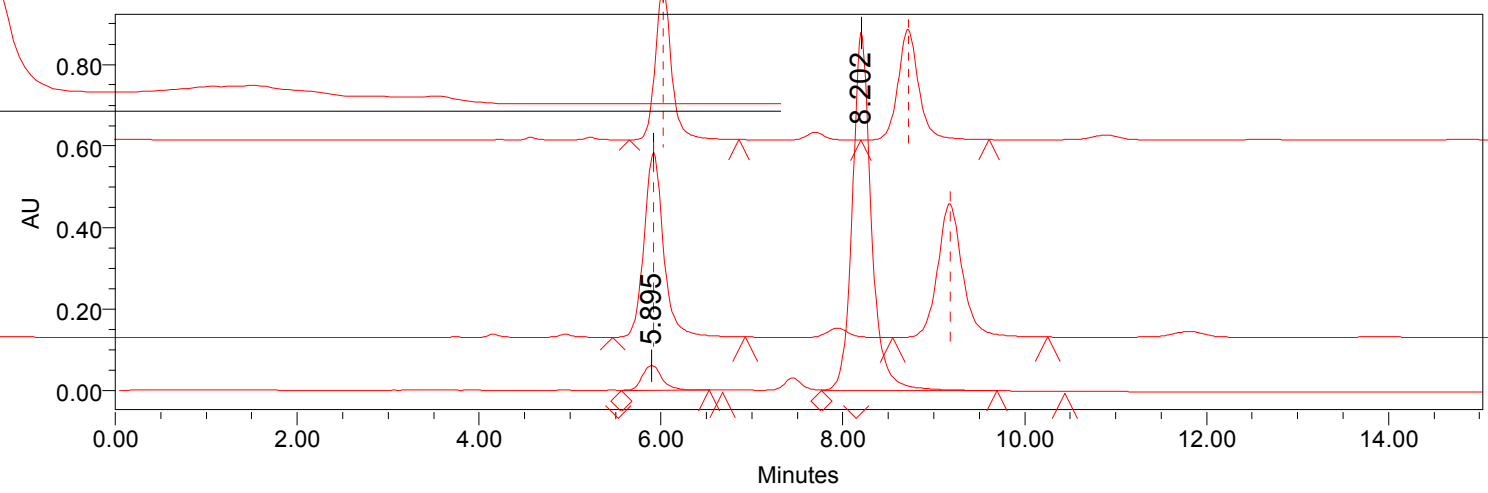

Processed Channel: PDA $220.0 \mathrm{~nm}$

\begin{tabular}{|r|l|r|r|r|r|}
\hline & Processed Channel & $\begin{array}{r}\text { Retention } \\
\text { Time (min) }\end{array}$ & Area & \% Area & Height \\
\hline 1 & PDA $220.0 \mathrm{~nm}$ & 5.895 & 923944 & 6.68 & 60591 \\
\hline 2 & PDA $220.0 \mathrm{~nm}$ & 8.202 & 12907615 & 93.32 & 879733 \\
\hline
\end{tabular}


${ }^{1} \mathrm{H}$ NMR $\left(400 \mathrm{MHz}, \mathrm{CDCl}_{3}\right)$ of $\left(S_{a}, R\right)-\mathbf{8 d}$ :

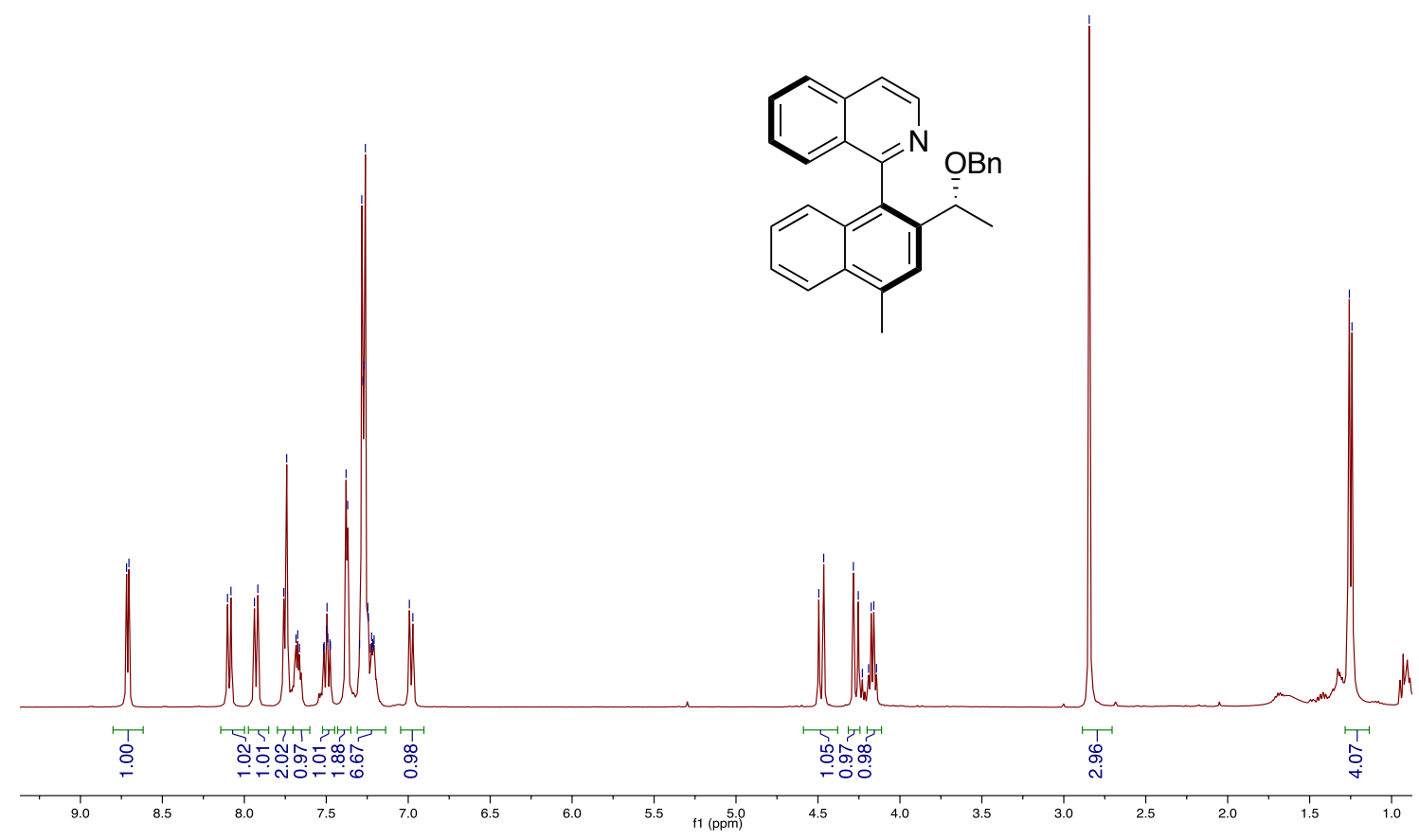

${ }^{13} \mathrm{C}$ NMR $\left(100 \mathrm{MHz}, \mathrm{CDCl}_{3}\right)$ of $\left(S_{a}, R\right)-\mathbf{8 d}$ :
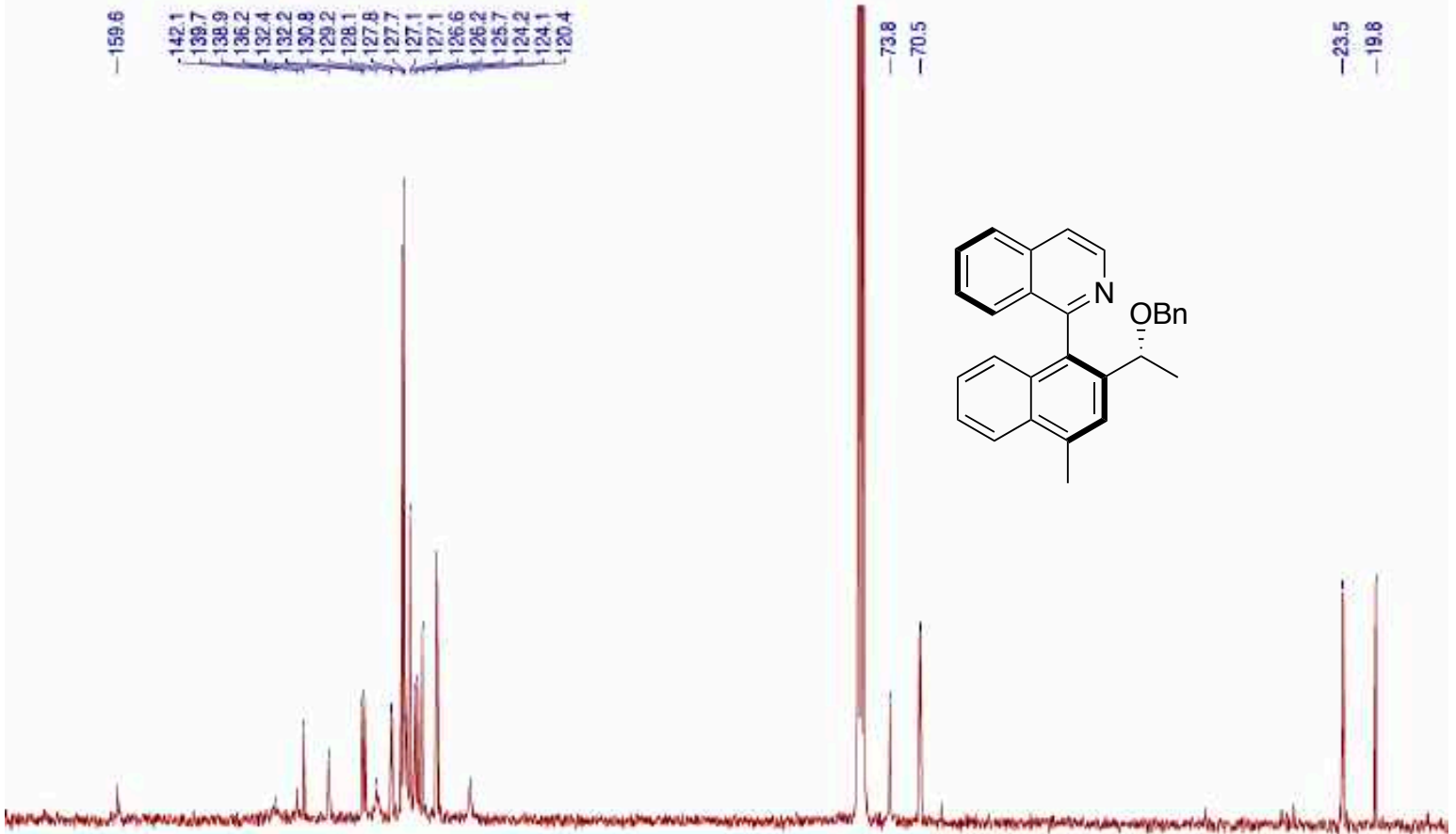
Racemic sample of $( \pm)$-8d: OD column, $n$-hexane:isopropanol 90:10, F= $1 \mathrm{~mL} / \mathrm{min}, \mathrm{T}=$ $30^{\circ} \mathrm{C}$.

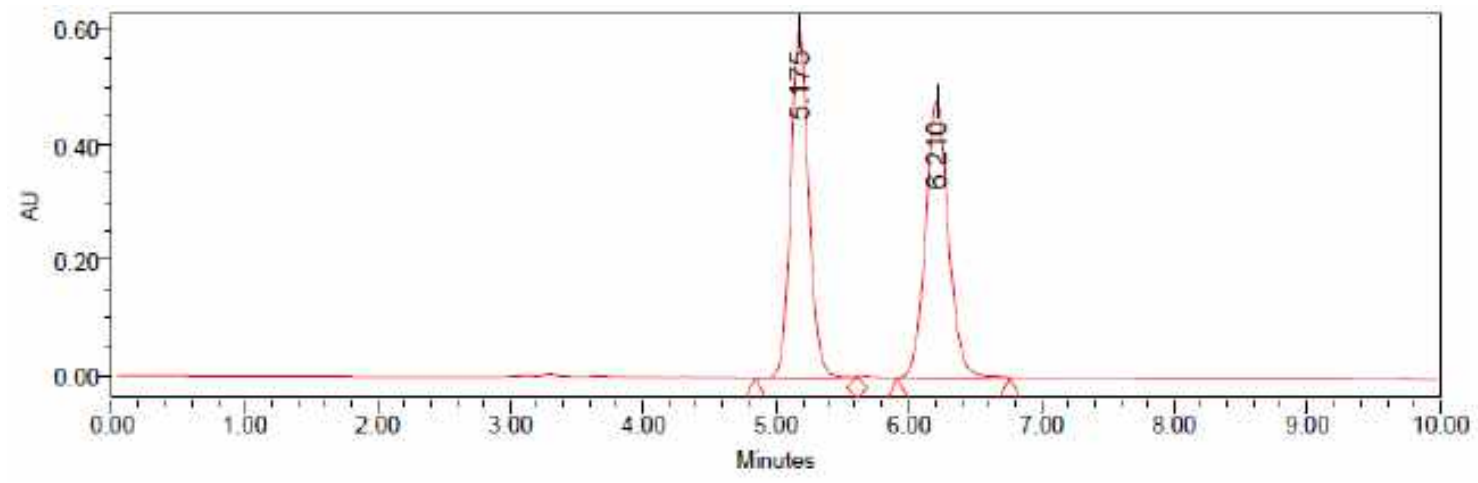

Processed Channel: PDA 220.0 nm
\begin{tabular}{|l|l|r|r|r|r|}
\hline & Processed Channel & $\begin{array}{l}\text { Retention } \\
\text { Time (min) }\end{array}$ & Area & $\%$ Area & Height \\
\hline 1 & PDA $220.0 \mathrm{~nm}$ & 5.175 & 5652378 & 49.56 & 603015 \\
\hline 2 & PDA $220.0 \mathrm{~nm}$ & 6.210 & 5753563 & 50.44 & 479523 \\
\hline
\end{tabular}

Enantioriched sample of $\left(S_{a}, R\right)-\mathbf{8 d}$ :

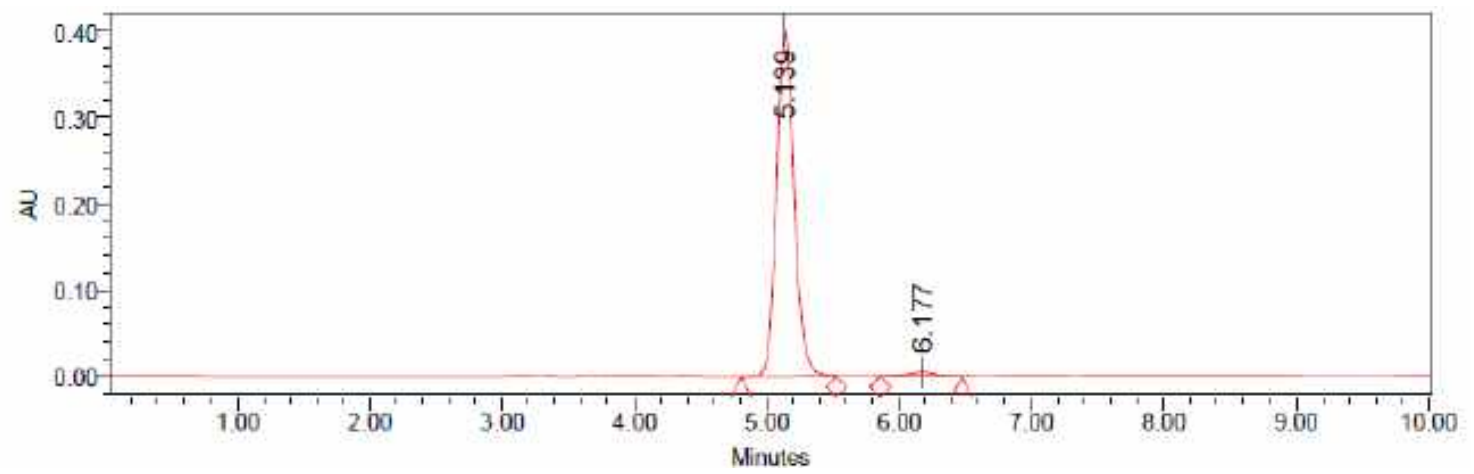

Processed Channel: PDA $220.0 \mathrm{~nm}$

\begin{tabular}{|l|l|r|r|r|r|}
\hline & Processed Channel & $\begin{array}{r}\text { Retention } \\
\text { Time (min) }\end{array}$ & Area & $\%$ Area & Height \\
\hline 1 & PDA 220.0 nm & 5.139 & 3738693 & 97.89 & 402160 \\
\hline 2 & PDA 220.0 nm & 6.177 & 80520 & 2.11 & 5373 \\
\hline
\end{tabular}


${ }^{1} \mathrm{H}$ NMR $\left(400 \mathrm{MHz}, \mathrm{CDCl}_{3}\right)$ of $\left(S_{a}, R\right)-\mathbf{8 e}$ :

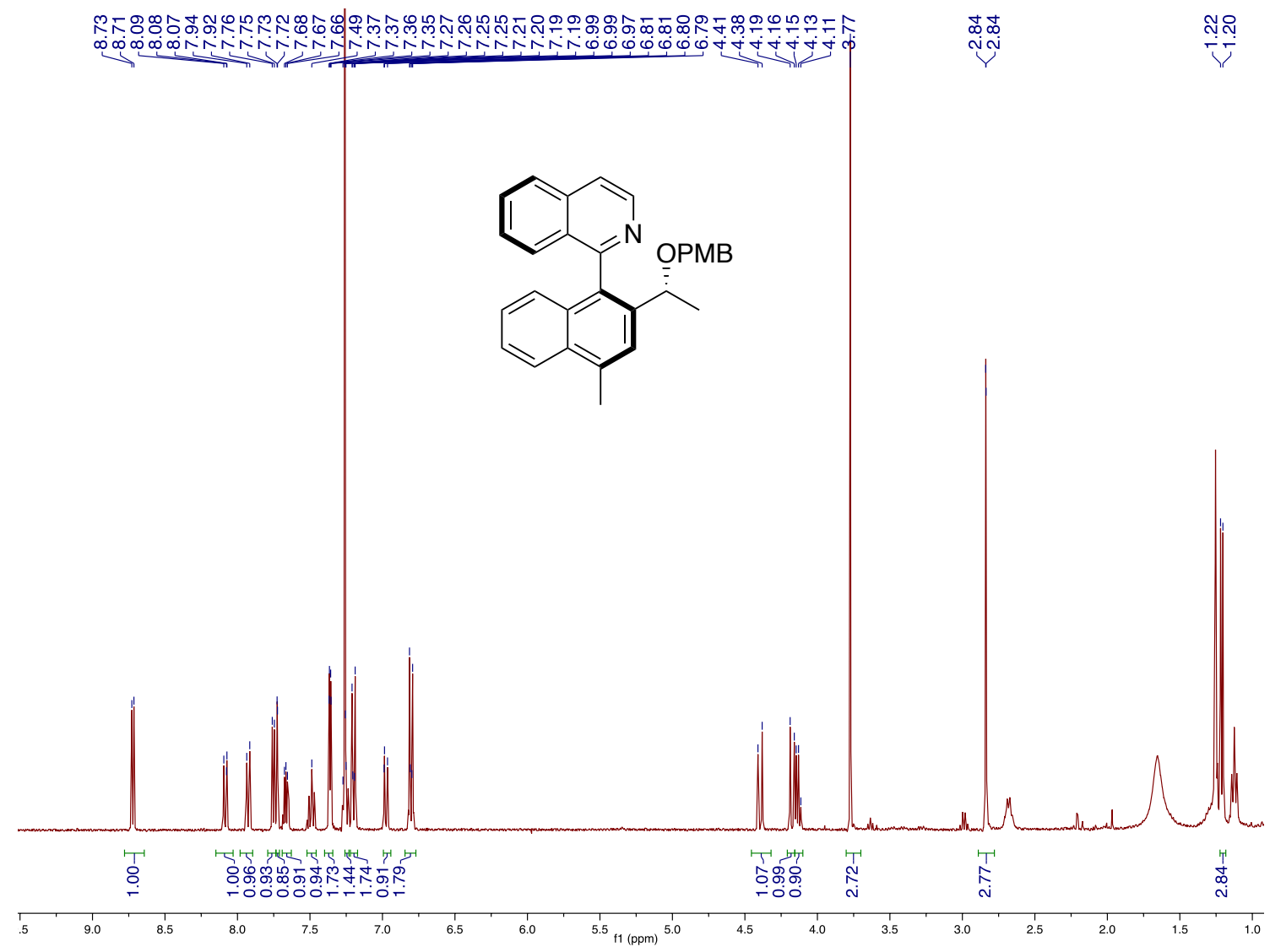

${ }^{13} \mathrm{C}$ NMR $\left(100 \mathrm{MHz}, \mathrm{CDCl}_{3}\right)$ of $\left(S_{a}, R\right)-\mathbf{8 e}$ :

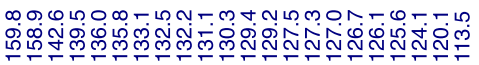

(1).....

迎 苍

$\stackrel{+}{\stackrel{\infty}{i} \stackrel{\infty}{\sigma}}$
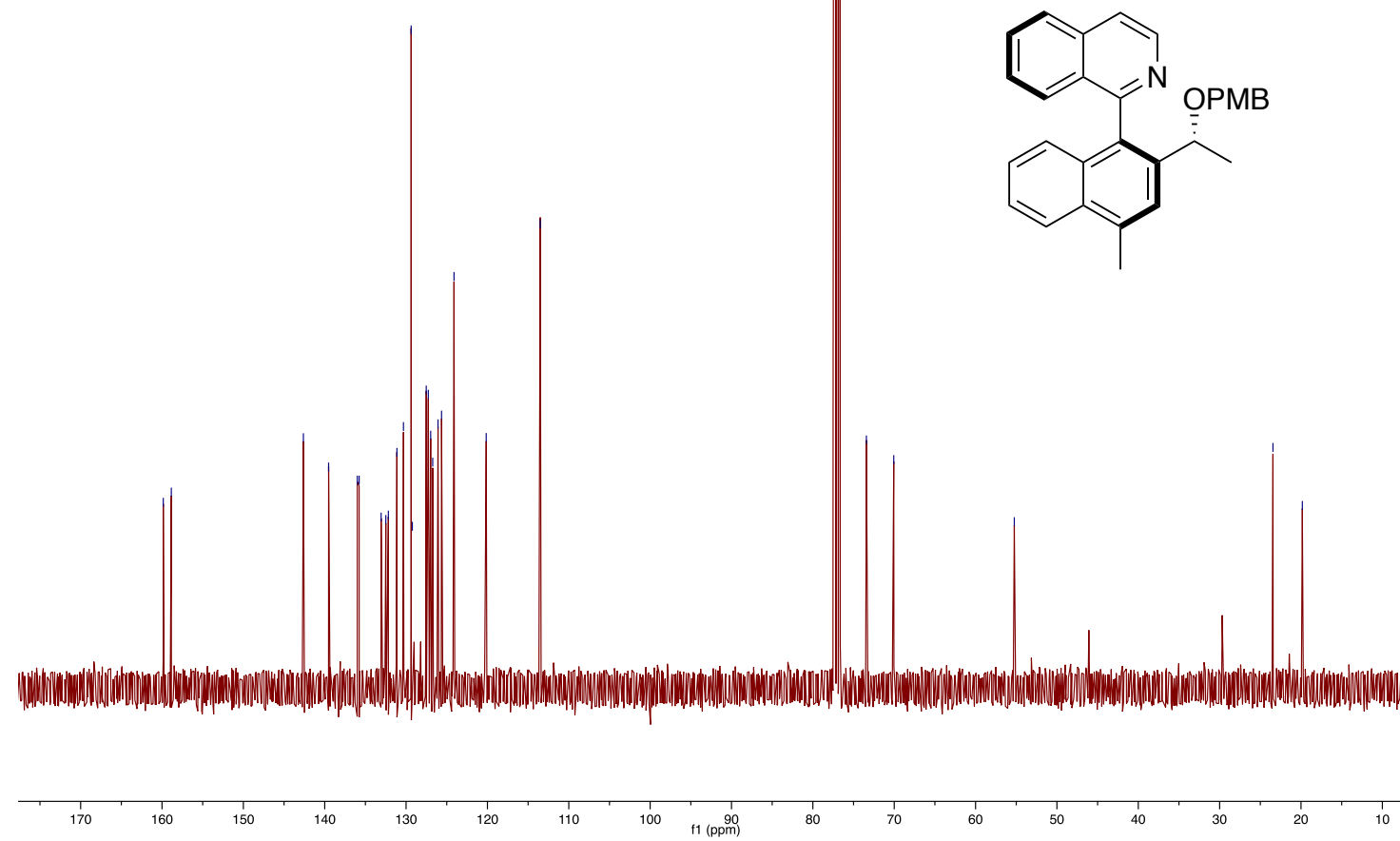
Racemic sample of $( \pm)-8 e$ : OD column, $n$-hexane:isopropanol 90:10, F= $1 \mathrm{~mL} / \mathrm{min}, \mathrm{T}=$ $30^{\circ} \mathrm{C}$.

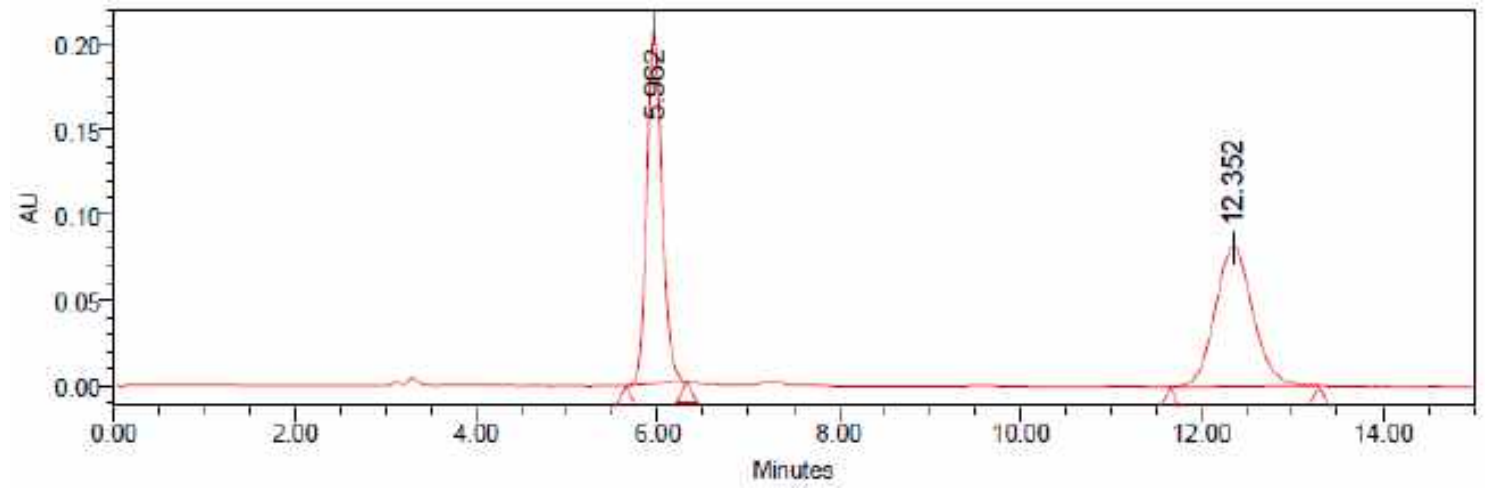

Processed Channel: PDA $221.5 \mathrm{~nm}$

\begin{tabular}{|l|l|r|r|r|r|}
\hline & Processed Channel & $\begin{array}{c}\text { Retention } \\
\text { Time (min) }\end{array}$ & Area & $\%$ Area & Height \\
\hline 1 & PDA $221.5 \mathrm{~nm}$ & 5.962 & 2385872 & 50.28 & 208679 \\
\hline 2 & PDA 221.5 nm & 12.352 & 2359745 & 49.72 & 81686 \\
\hline
\end{tabular}

Enantioriched sample of $\left(S_{a}, R\right)$ - 8e:

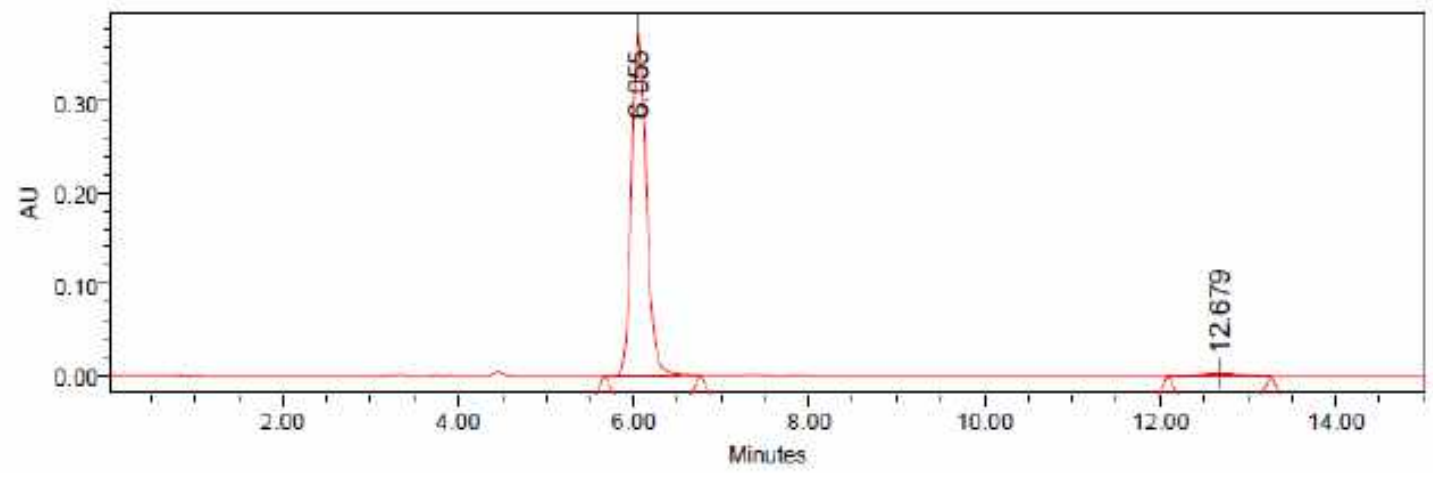

\section{Processed Channel: PDA $221.5 \mathrm{~nm}$}

\begin{tabular}{|l|l|r|r|r|r|}
\hline & Processed Channel & $\begin{array}{r}\text { Retention } \\
\text { Time }(\mathrm{min})\end{array}$ & Area & \% Area & Height \\
\hline 1 & PDA 221.5 nm & 6.055 & 4338842 & 98.57 & 378888 \\
\hline 2 & PDA 221.5 nm & 12.679 & 63071 & 1.43 & 2216 \\
\hline
\end{tabular}


${ }^{1} \mathrm{H}$ NMR $\left(400 \mathrm{MHz}, \mathrm{CDCl}_{3}\right)$ of $\left(S_{a}, R\right)-\mathbf{8 f}$ :

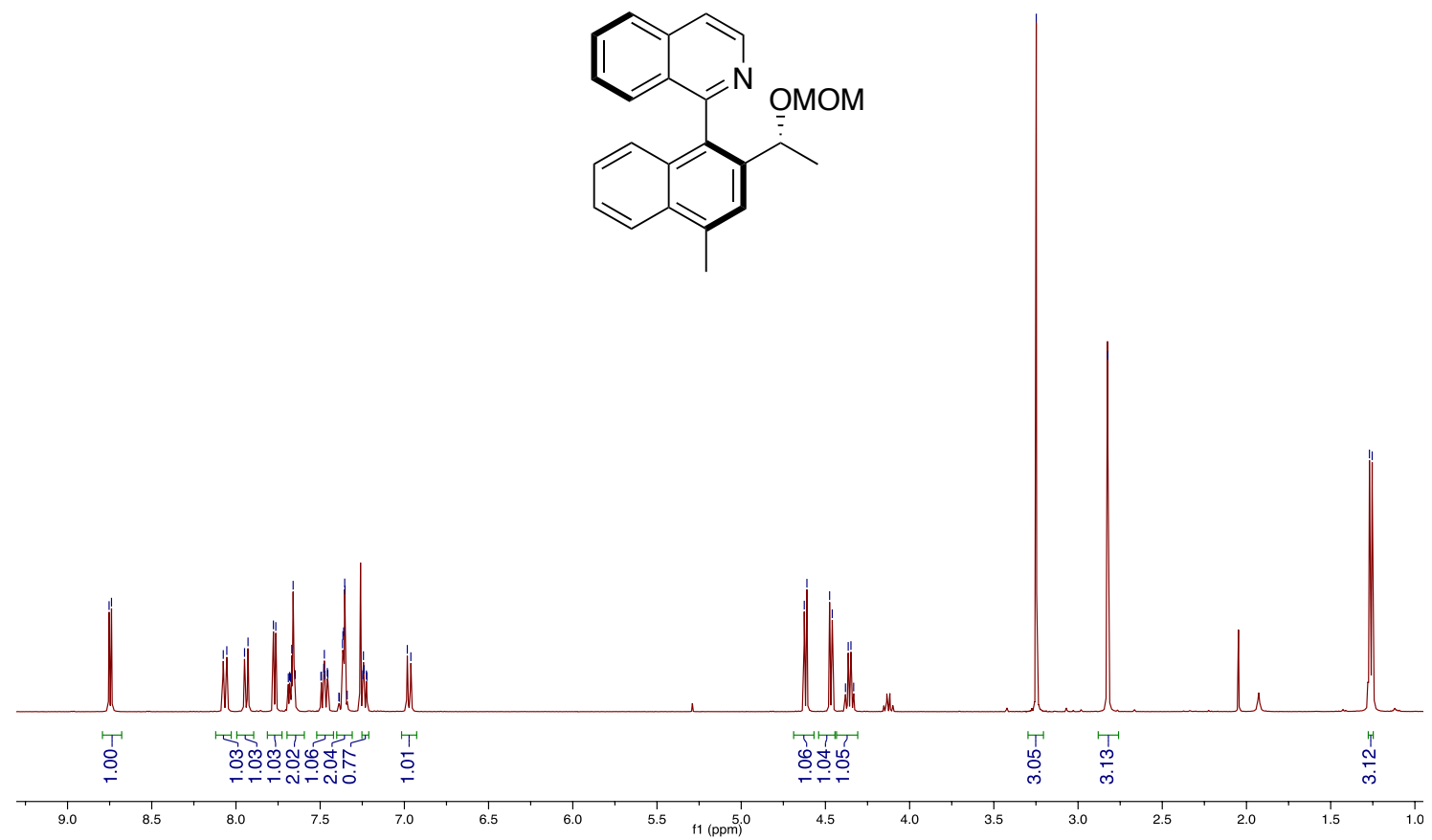

${ }^{13} \mathrm{C}$ NMR $\left(100 \mathrm{MHz}, \mathrm{CDCl}_{3}\right)$ of $\left(S_{a}, R\right)-\mathbf{8 f}$ :

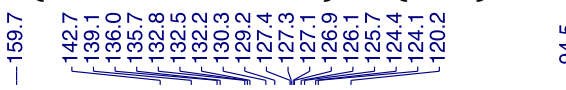

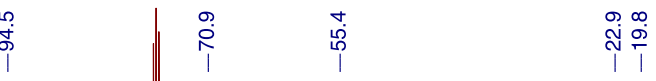

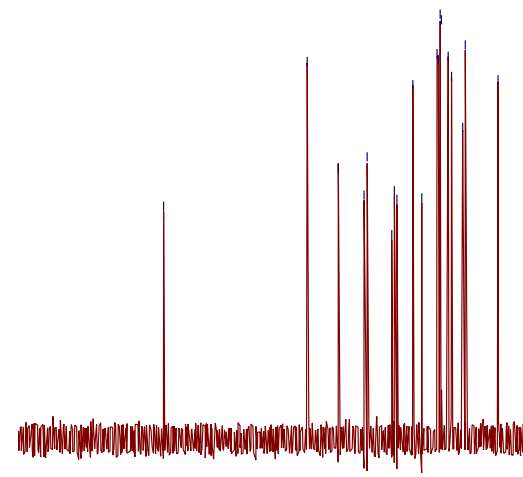<smiles>COC(C)c1cc(C)c2ccccc2c1-c1nccc2ccccc12</smiles> 
Racemic sample of $( \pm)$-8f: IA column, $n$-hexane:isopropanol 95:5, F= $1 \mathrm{~mL} / \mathrm{min}, \mathrm{T}=$ $30^{\circ} \mathrm{C}$.

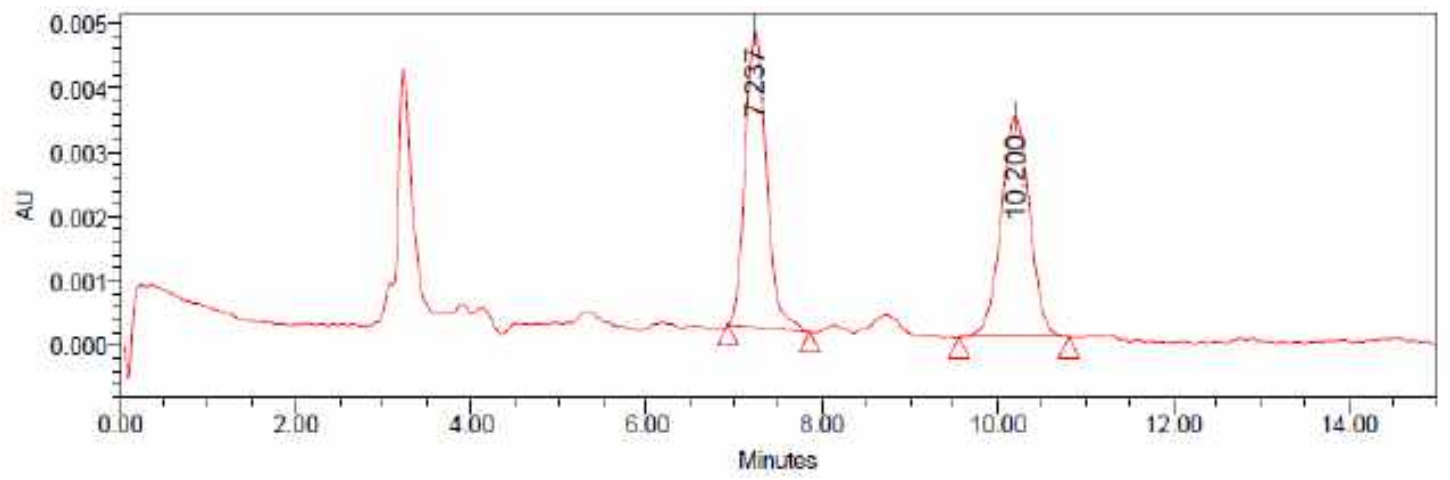

Processed Channel: PDA $220.0 \mathrm{~nm}$

\begin{tabular}{|l|l|r|r|r|r|}
\hline & Processed Channel & $\begin{array}{c}\text { Retention } \\
\text { Time }(\mathrm{min})\end{array}$ & Area & \% Area & Height \\
\hline 1 & PDA 220.0 nm & 7.237 & 77891 & 49.96 & 4623 \\
\hline 2 & PDA 220.0 nm & 10.200 & 78015 & 50.04 & 3444 \\
\hline
\end{tabular}

Enantioriched sample of $\left(S_{a}, R\right)$ - 8f:

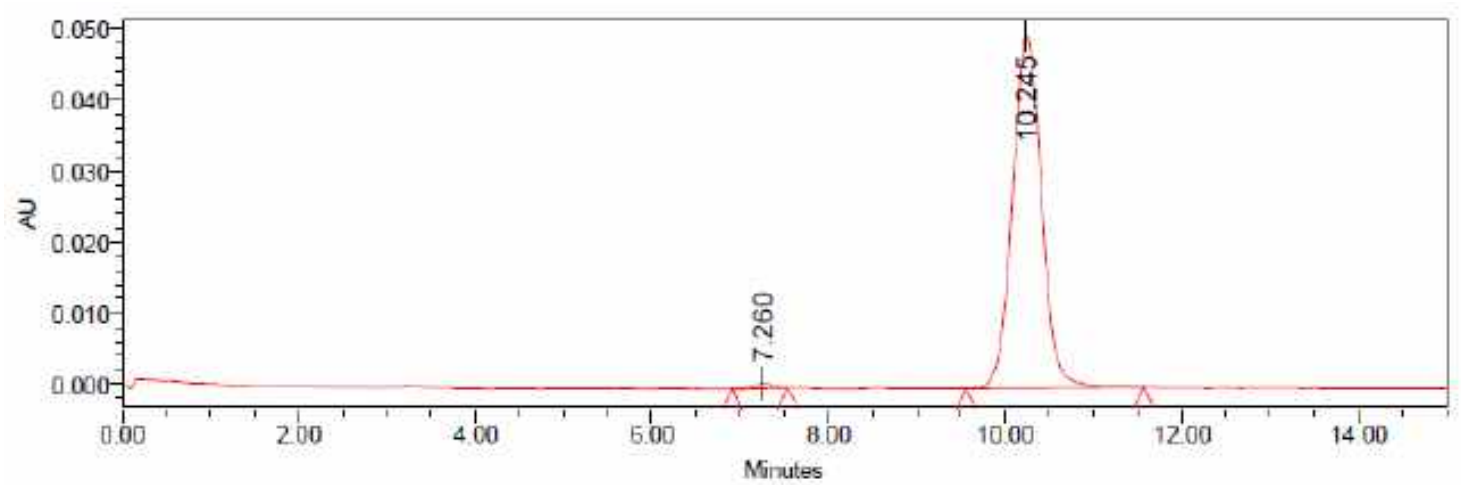

\section{Processed Channel: PDA $220.0 \mathrm{~nm}$}

\begin{tabular}{|r|l|r|r|r|r|}
\hline & Processed Channel & $\begin{array}{r}\text { Retention } \\
\text { Time }(\mathrm{min})\end{array}$ & Area & \% Area & Height \\
\hline 1 & PDA 220.0 nm & 7.260 & 10762 & 0.94 & 713 \\
\hline 2 & PDA 220.0 nm & 10.245 & 1132871 & 99.06 & 49400 \\
\hline
\end{tabular}


${ }^{1} \mathrm{H}$ NMR (400 MHz, $\mathrm{CDCl}_{3}$ ) of the minor diastereomer of $\mathbf{8 g}$ :

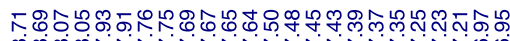

Y

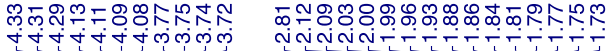<smiles>Cc1cc(C2CCCO2)c(-c2nccc3ccccc23)c2ccccc12</smiles>

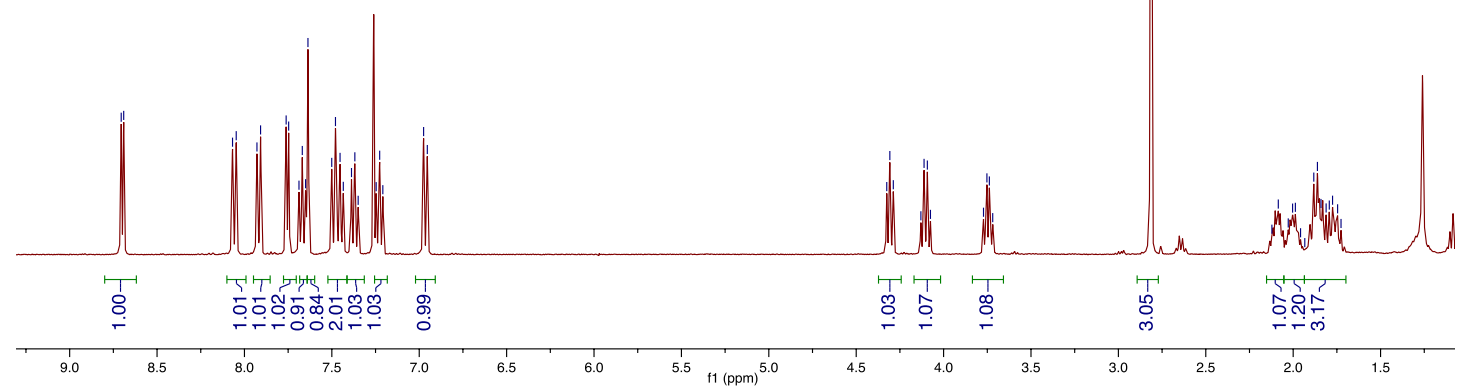

${ }^{13} \mathrm{C} \mathrm{NMR}\left(100 \mathrm{MHz}, \mathrm{CDCl}_{3}\right)$ of the minor diastereomer of $\mathbf{8 g}$ :

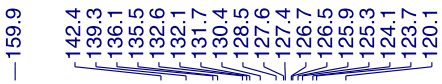

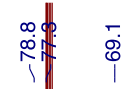

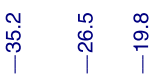<smiles>Cc1cc(C2CCCO2)c(-c2nccc3ccccc23)c2ccccc12</smiles>

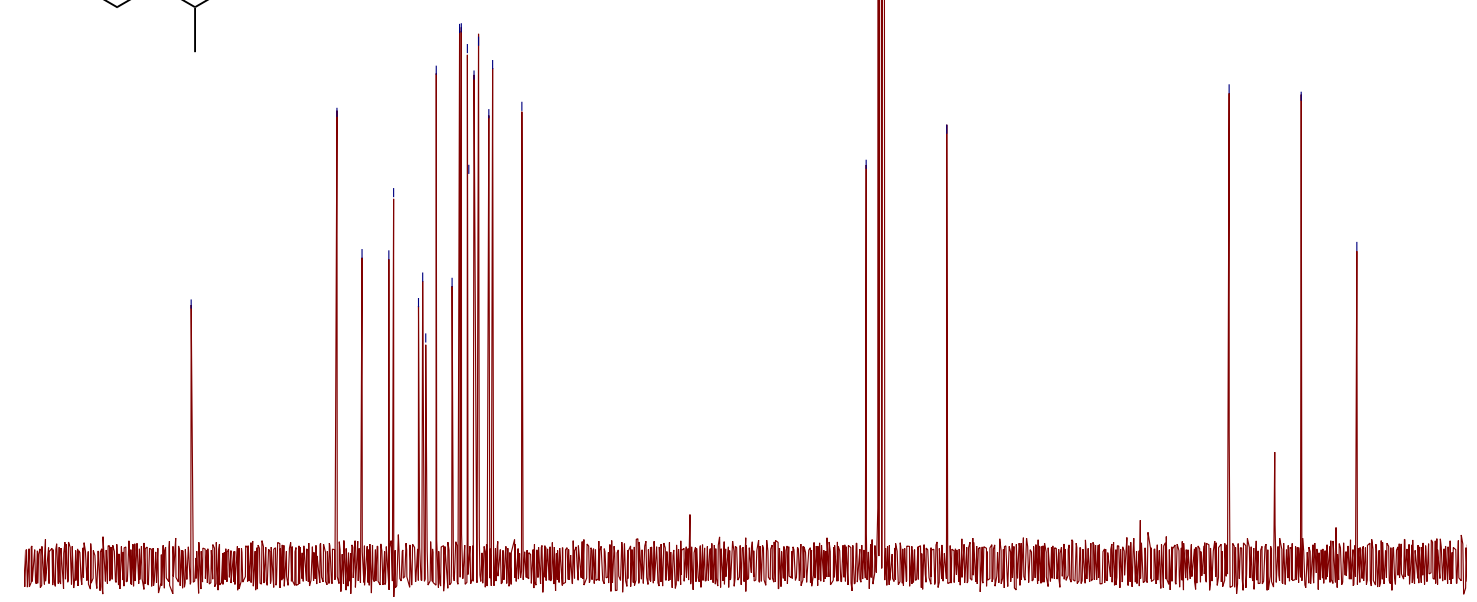

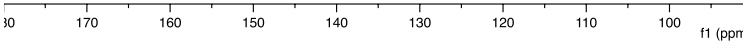


Racemic sample $( \pm$ )-minor diastereomer of 8g: OD-H column, $n$-hexane:isopropanol $90: 10, \mathrm{~F}=1 \mathrm{~mL} / \mathrm{min}, \mathrm{T}=30^{\circ} \mathrm{C}$.

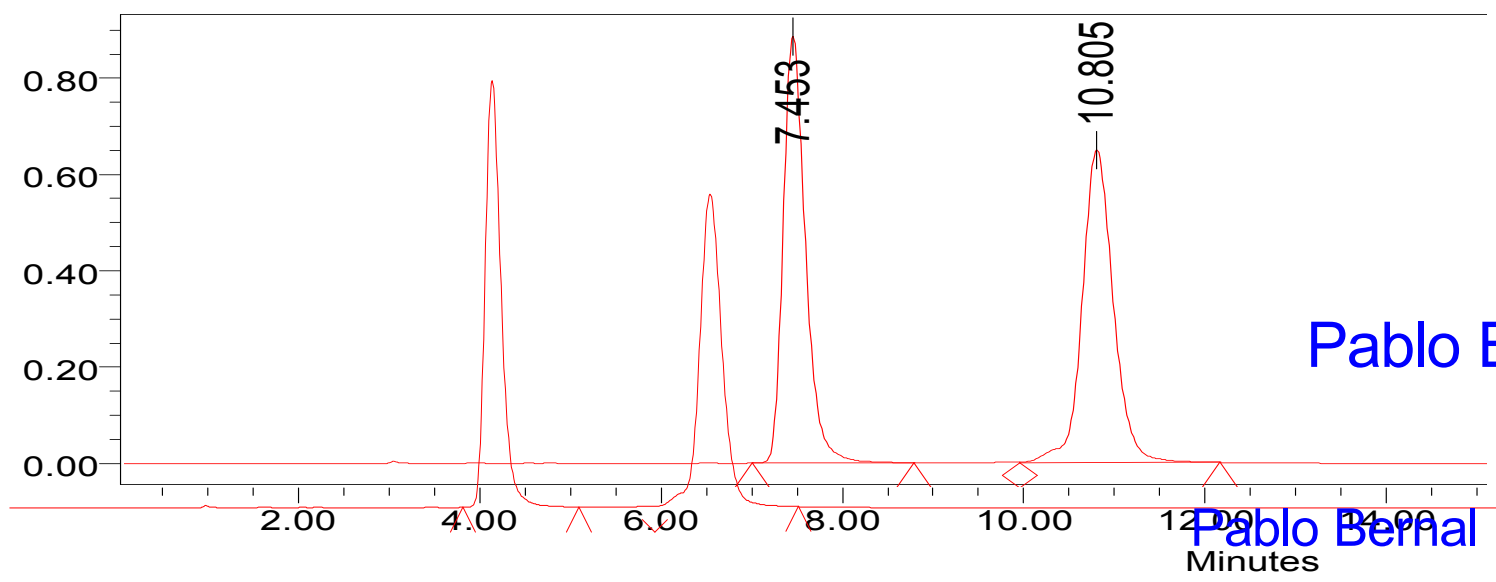

\section{Processed Channel: PDA $222.0 \mathrm{~nm}$}

\begin{tabular}{|r|l|r|r|r|r|}
\hline & Processed Channel & $\begin{array}{r}\text { Retention } \\
\text { Time }(\min )\end{array}$ & Area & \% Area & Height \\
\hline 1 & PDA 222.0 nm & 7.453 & 15132332 & 49.06 & 888107 \\
\hline 2 & PDA 222.0 nm & 10.805 & 15710510 & 50.94 & 650341 \\
\hline
\end{tabular}

\section{Enantioriched sample of the minor diastereomer of $8 \mathrm{~g}$ :}

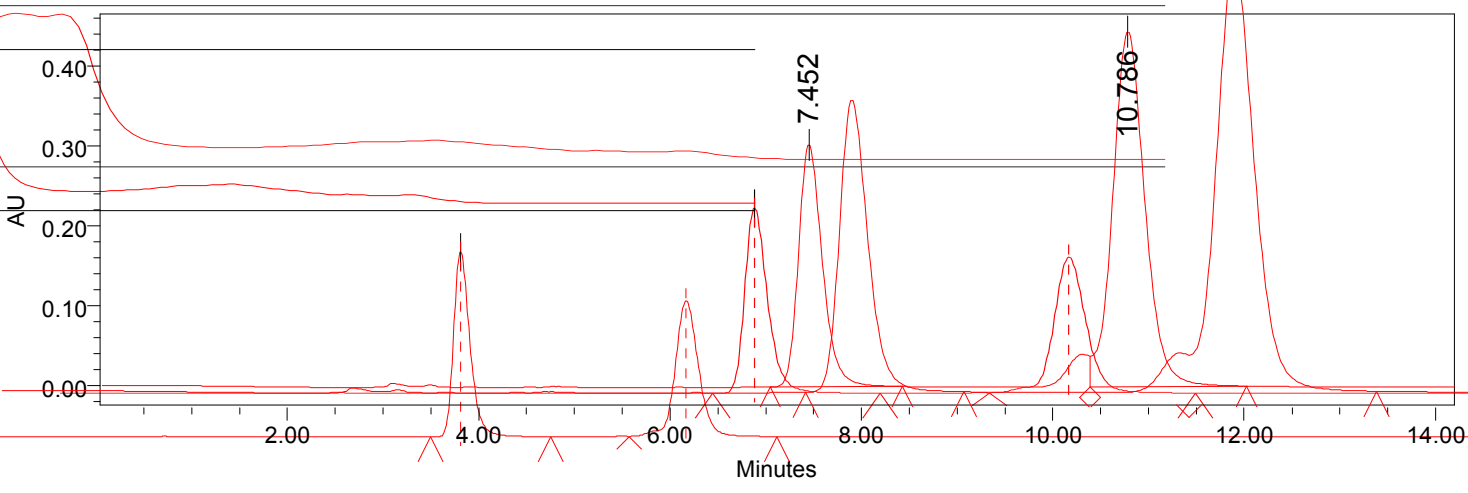

Processed Channel: PDA 225.7 nm

\begin{tabular}{|r|l|r|r|r|r|}
\hline & Processed Channel & $\begin{array}{r}\text { Retention } \\
\text { Time }(\mathrm{min})\end{array}$ & \multicolumn{1}{|c|}{ Area } & \% Area & Height \\
\hline 1 & PDA 225.7 nm & 7.452 & 5282319 & 33.71 & 303685 \\
\hline 2 & PDA 225.7 nm & 10.786 & 10389384 & 66.29 & 444977 \\
\hline
\end{tabular}


${ }^{1} \mathrm{H}$ NMR $\left(400 \mathrm{MHz}, \mathrm{CDCl}_{3}\right)$ of $\left(R_{a}, S\right)-\mathbf{8 g}$ :

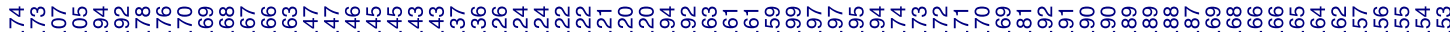

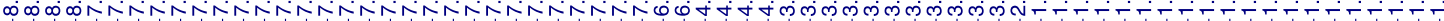<smiles>Cc1cc([C@H]2CCCO2)c(-c2nccc3ccccc23)c2ccccc12</smiles>

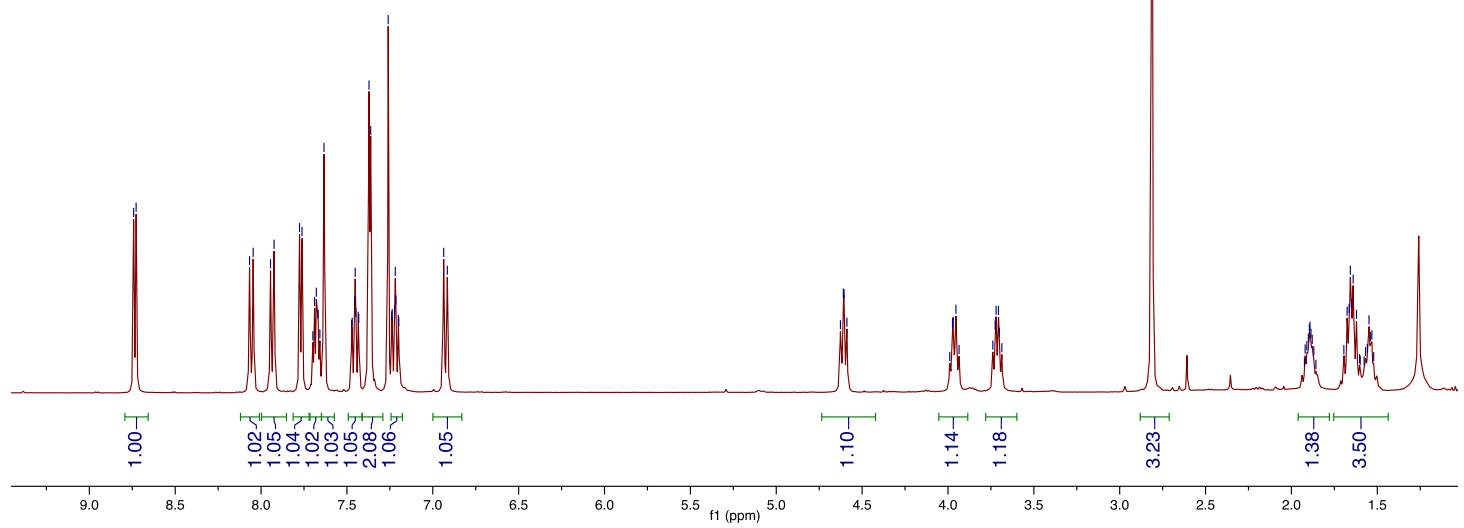

${ }^{13} \mathrm{C}$ NMR $\left(100 \mathrm{MHz} \mathrm{CDCl}_{3}\right)$ of $\left(R_{a}, S\right)-\mathbf{8 g}$ :

:

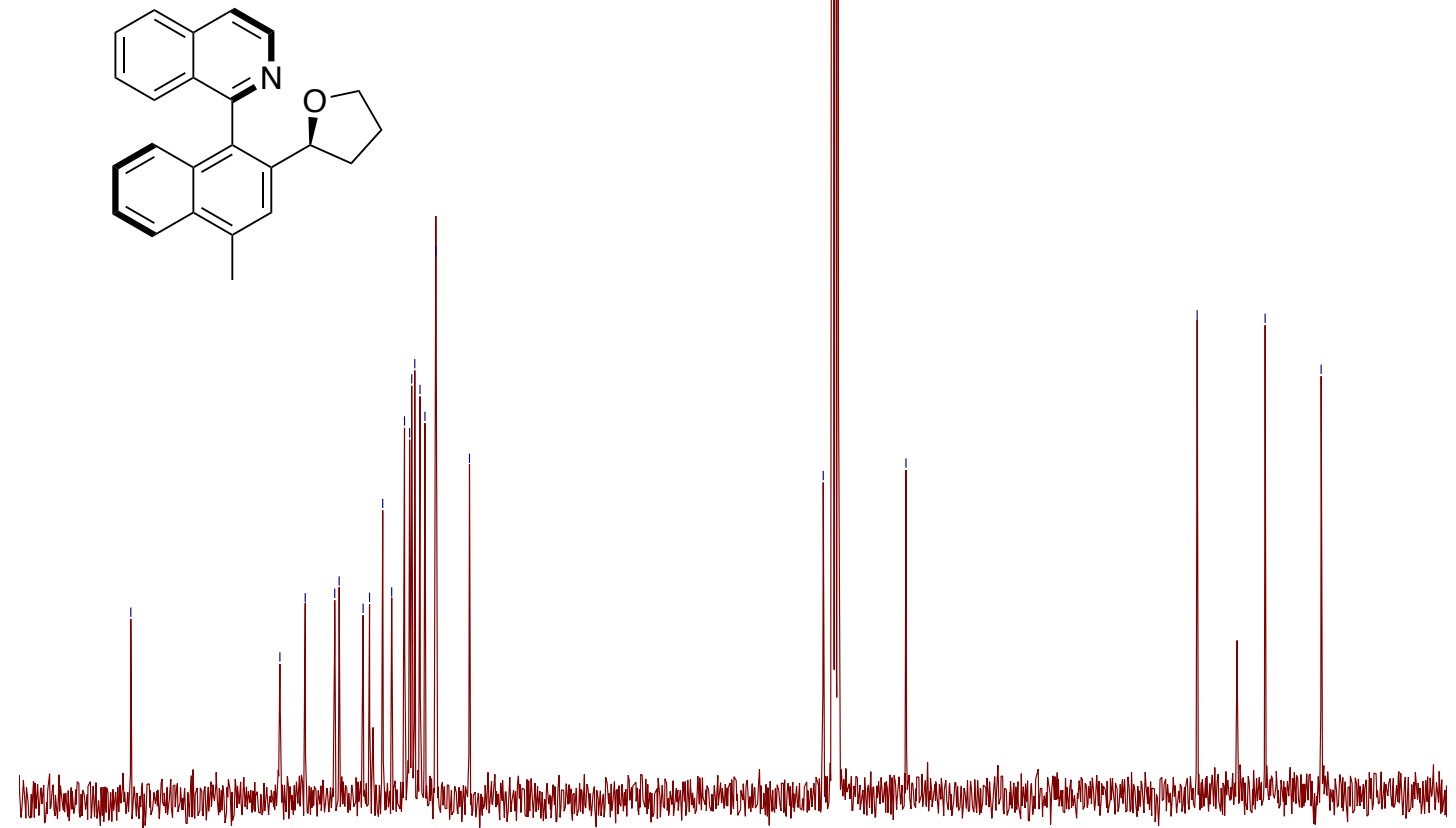

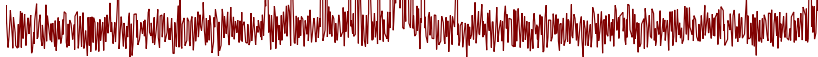
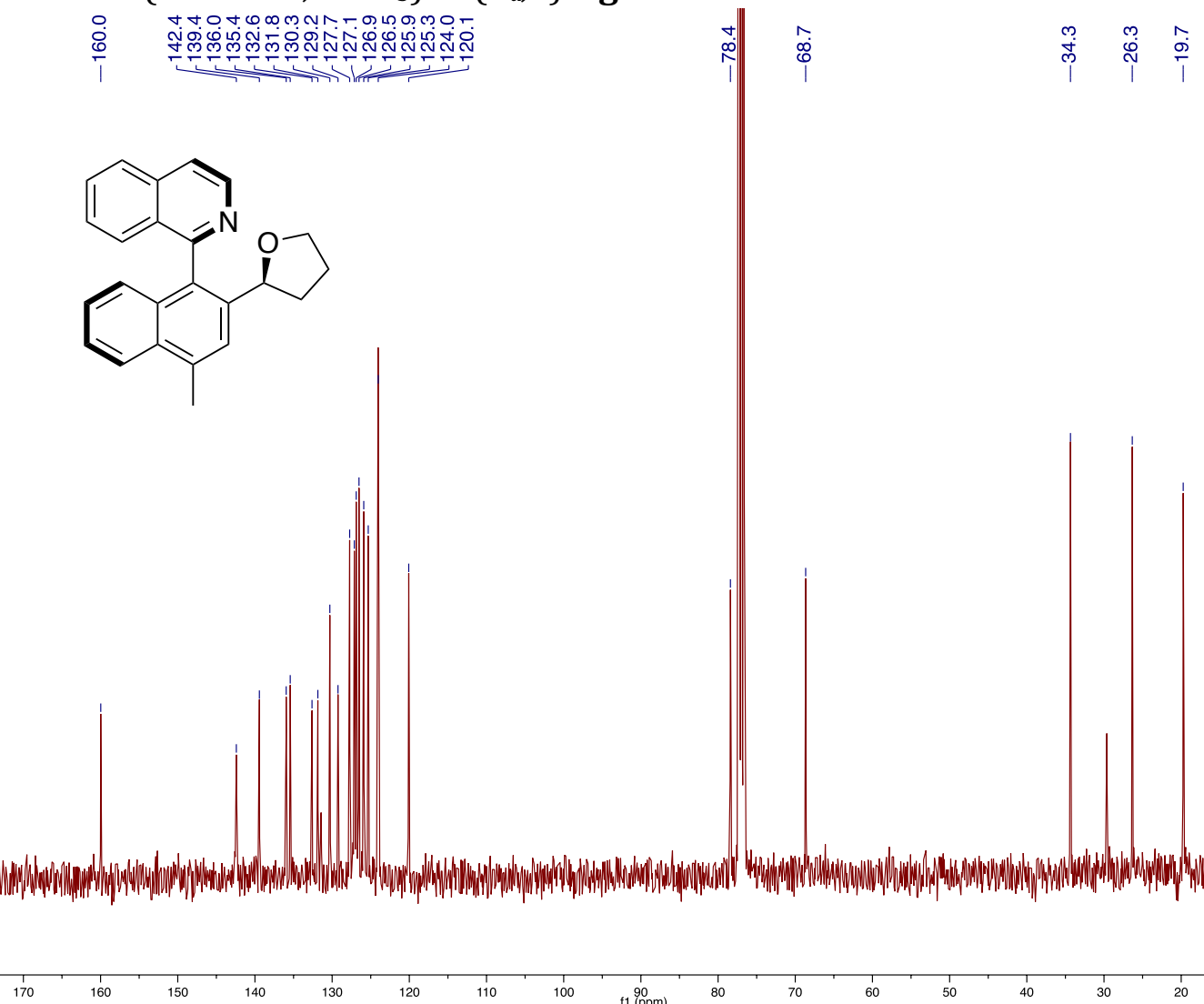
Racemic sample $( \pm)$-major diastereomer of 8g: IA column, $n$-hexane:isopropanol $90: 10, \mathrm{~F}=1 \mathrm{~mL} / \mathrm{min}, \mathrm{T}=30^{\circ} \mathrm{C}$.

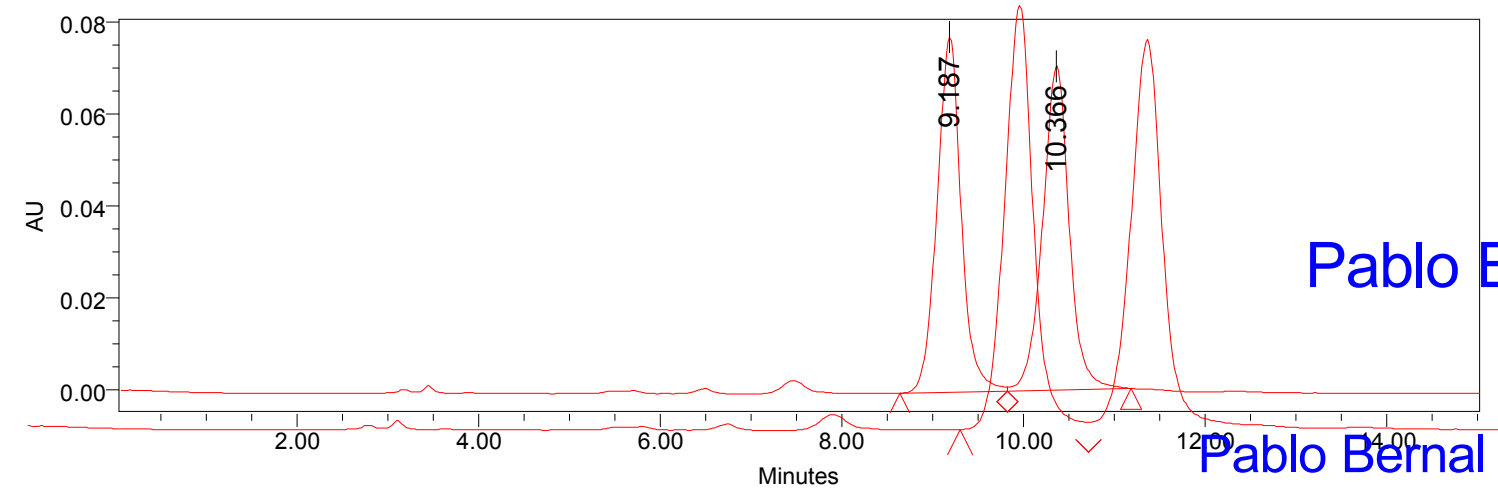

Processed Channel: PDA 220.0 nm
\begin{tabular}{|l|l|r|r|r|r|}
\hline & Processed Channel & $\begin{array}{c}\text { Retention } \\
\text { Time (min) }\end{array}$ & Area & $\%$ Area & Height \\
\hline 1 & PDA 220.0 nm & 9.187 & 1431770 & 49.97 & 77116 \\
\hline 2 & PDA 220.0 nm & 10.366 & 1433479 & 50.03 & 70414 \\
\hline
\end{tabular}

\section{Enantioriched sample of $\left(R_{a}, S\right)$-8g:}

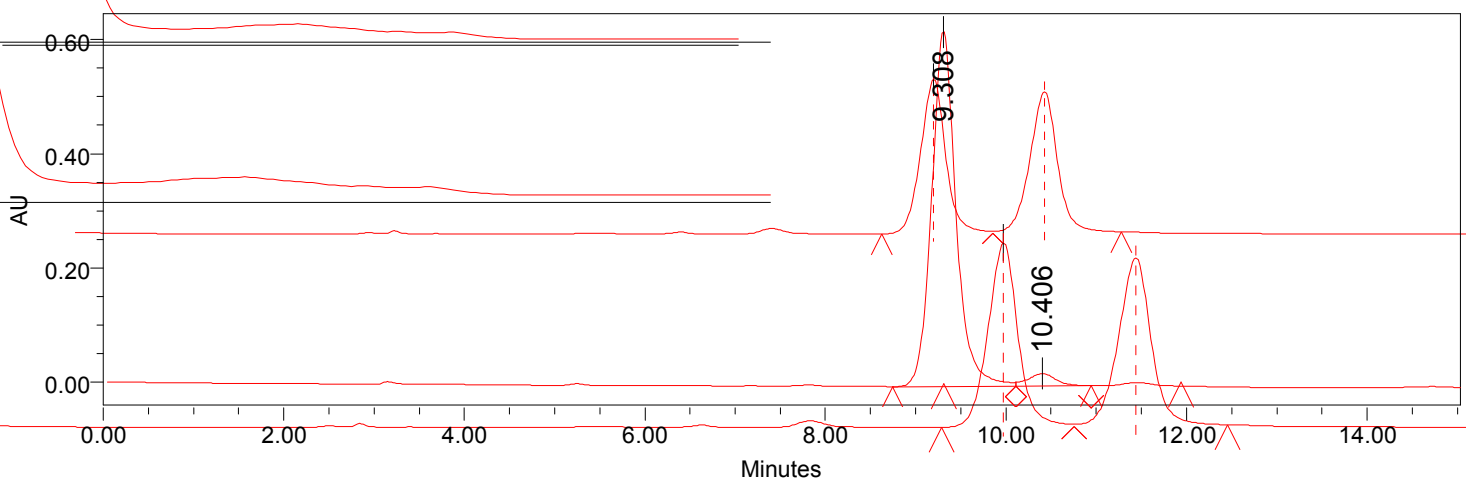

Processed Channel: PDA $231.9 \mathrm{~nm}$

\begin{tabular}{|r|l|r|r|r|r|}
\hline & Processed Channel & $\begin{array}{r}\text { Retention } \\
\text { Time (min) }\end{array}$ & Area & \% Area & Height \\
\hline 1 & PDA $231.9 \mathrm{~nm}$ & 9.308 & 11675192 & 96.24 & 621675 \\
\hline 2 & PDA $231.9 \mathrm{~nm}$ & 10.406 & 456396 & 3.76 & 21667 \\
\hline
\end{tabular}


${ }^{1} \mathrm{H}$ NMR $\left(400 \mathrm{MHz}, \mathrm{CDCl}_{3}\right)$ of $\left(S_{a}, R\right)-\mathbf{9 a}:$

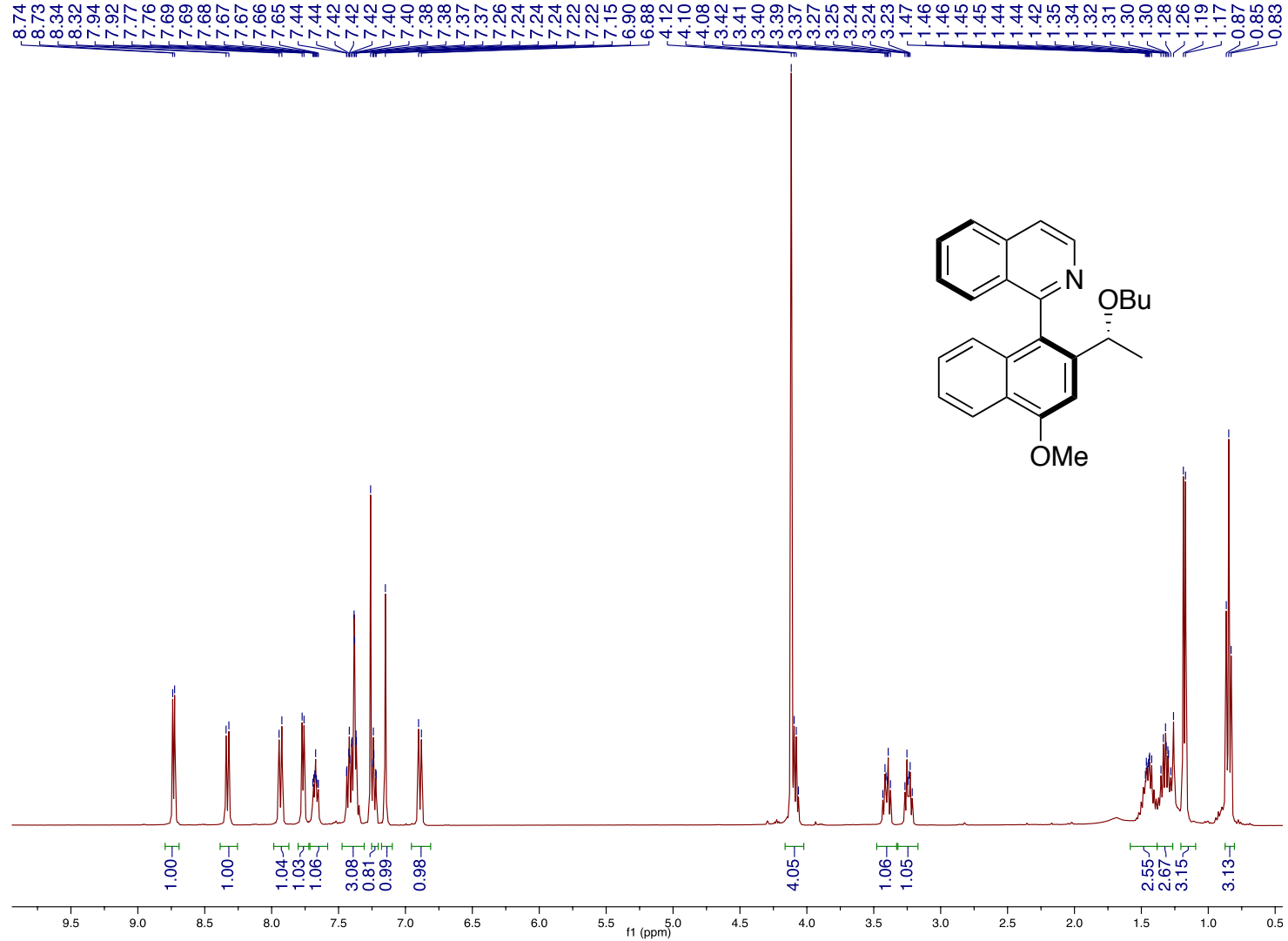

${ }^{13} \mathrm{C}$ NMR $\left(100 \mathrm{MHz}, \mathrm{CDCl}_{3}\right)$ of $\left(S_{a}, R\right)-\mathbf{9 a}$ :

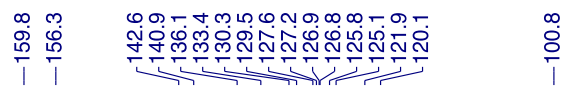

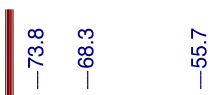

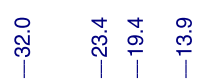

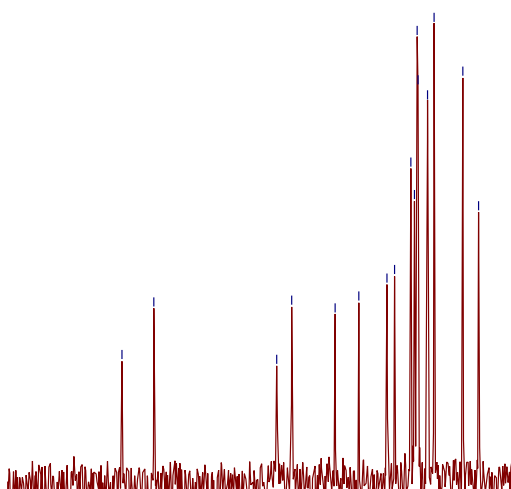<smiles>COc1cc(C(C)C)c(-c2nccc3ccccc23)c2ccccc12</smiles>

14.

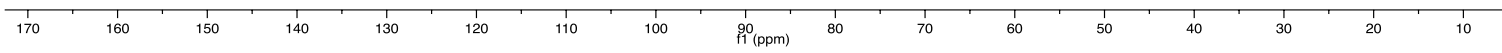


Racemic sample of $( \pm)$-9a: IA column, $n$-hexane:isopropanol 99:1, F= $1 \mathrm{~mL} / \mathrm{min}, \mathrm{T}=$ $30^{\circ} \mathrm{C}$.

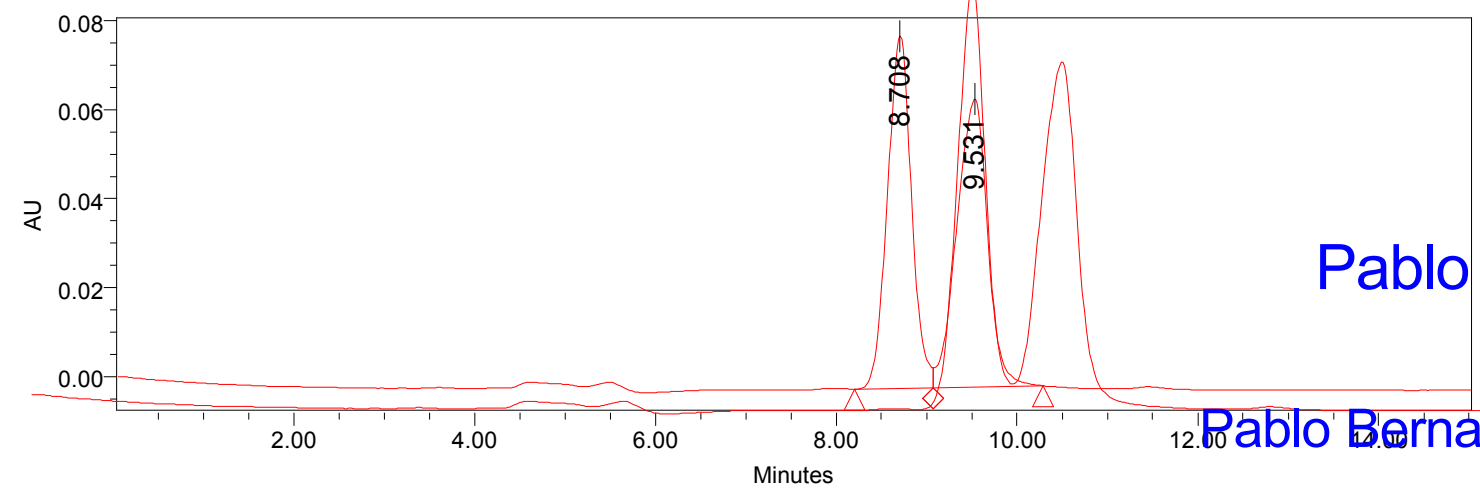

Processed Channel: PDA $220.8 \mathrm{~nm}$

\begin{tabular}{|l|l|r|r|r|r|}
\hline & Processed Channel & $\begin{array}{r}\text { Retention } \\
\text { Time }(\min )\end{array}$ & Area & \% Area & Height \\
\hline 1 & PDA $220.8 \mathrm{~nm}$ & 8.708 & 1470417 & 48.94 & 79268 \\
\hline 2 & PDA $220.8 \mathrm{~nm}$ & 9.531 & 1534009 & 51.06 & 64799 \\
\hline
\end{tabular}

Enantioriched sample of $\left(S_{a}, R\right)-9 a$ :

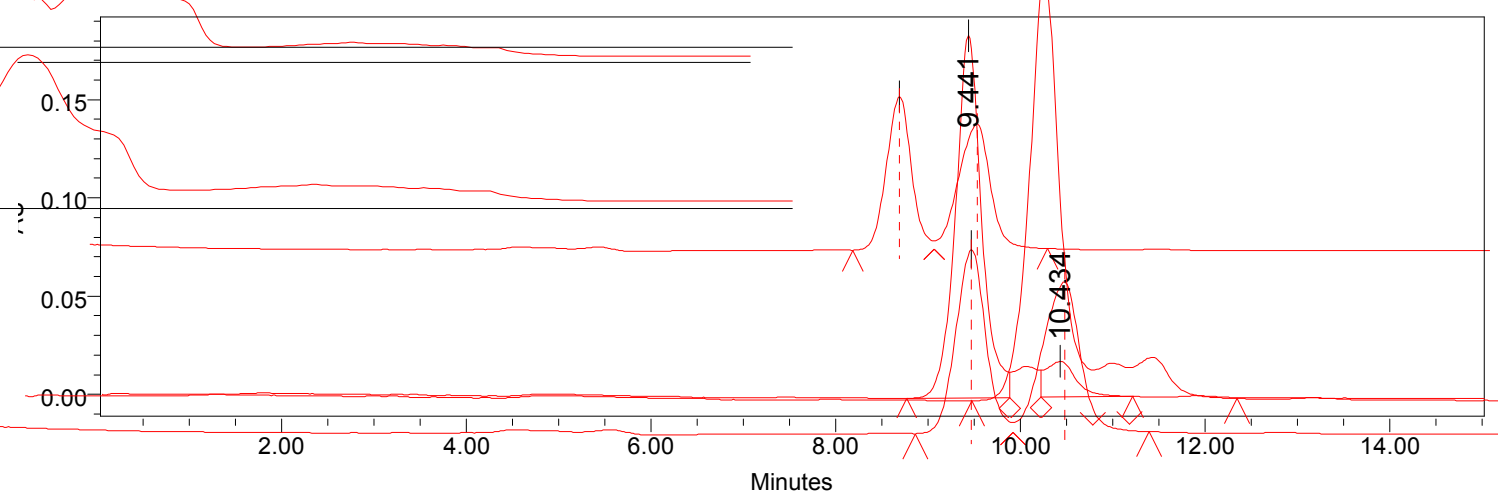

Processed Channel: PDA 212.4 nm

\begin{tabular}{|r|l|r|r|r|r|}
\hline & Processed Channel & $\begin{array}{r}\text { Retention } \\
\text { Time (min) }\end{array}$ & Area & \% Area & Height \\
\hline 1 & PDA 212.4 nm & 9.441 & 3631236 & 89.57 & 184493 \\
\hline 2 & PDA 212.4 nm & 10.434 & 422977 & 10.43 & 18205 \\
\hline
\end{tabular}


${ }^{1} \mathrm{H}$ NMR $\left(400 \mathrm{MHz}, \mathrm{CDCl}_{3}\right)$ of $\left(S_{a}, R\right)-9 \mathbf{b}:$

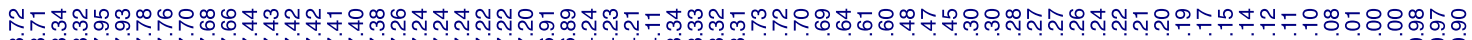

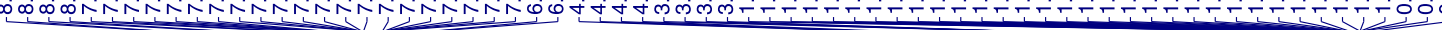

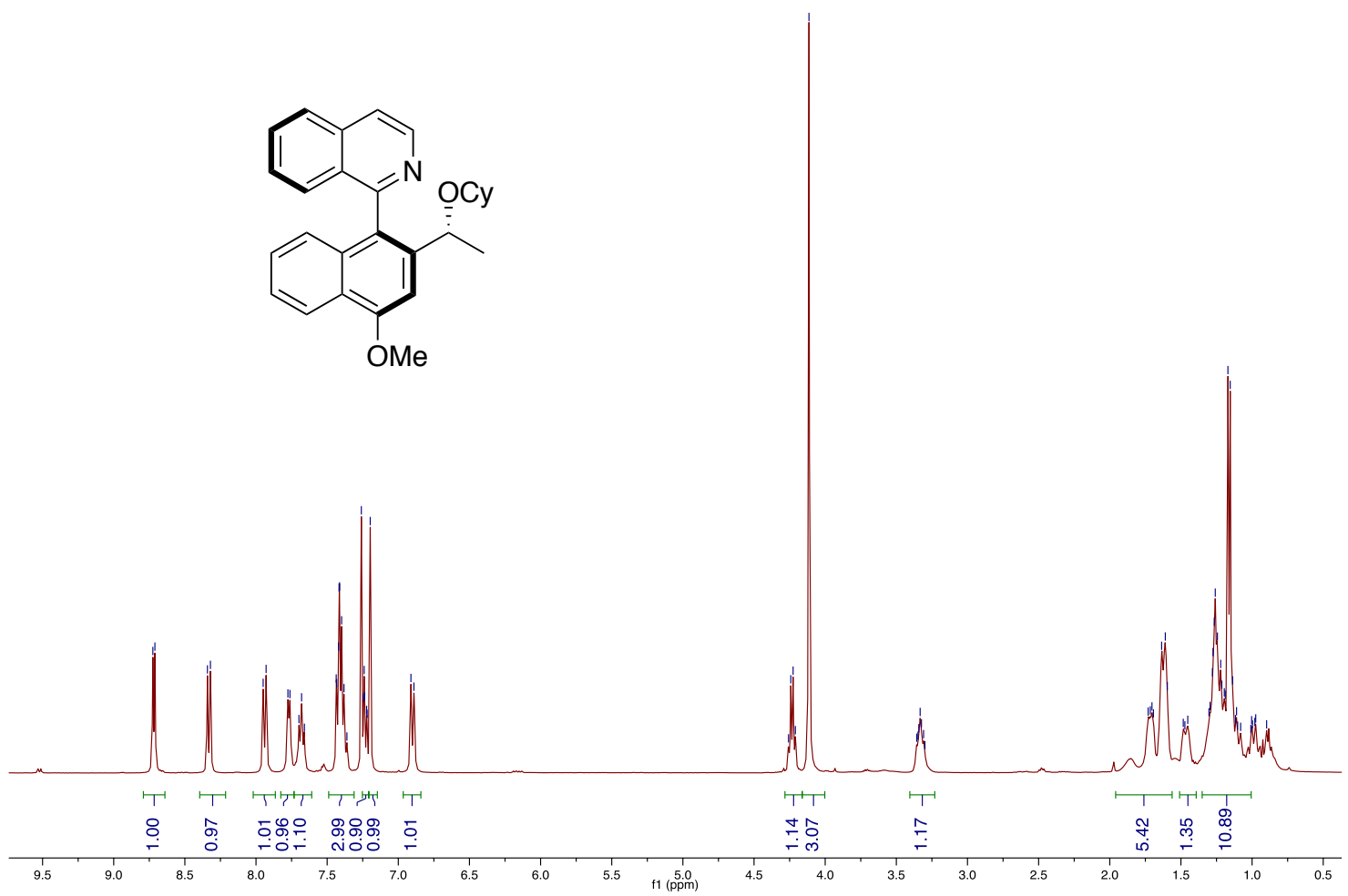

${ }^{13} \mathrm{C}$ NMR $\left(100 \mathrm{MHz}, \mathrm{CDCl}_{3}\right)$ of $\left(S_{a}, R\right)-\mathbf{9 b}$ :

은 금

$\frac{1}{i}$

驾

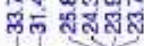<smiles>COc1cc(C(C)C)c(-c2nccc3ccccc23)c2ccccc12</smiles>

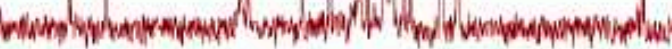

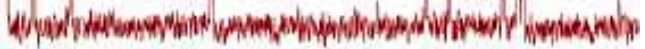
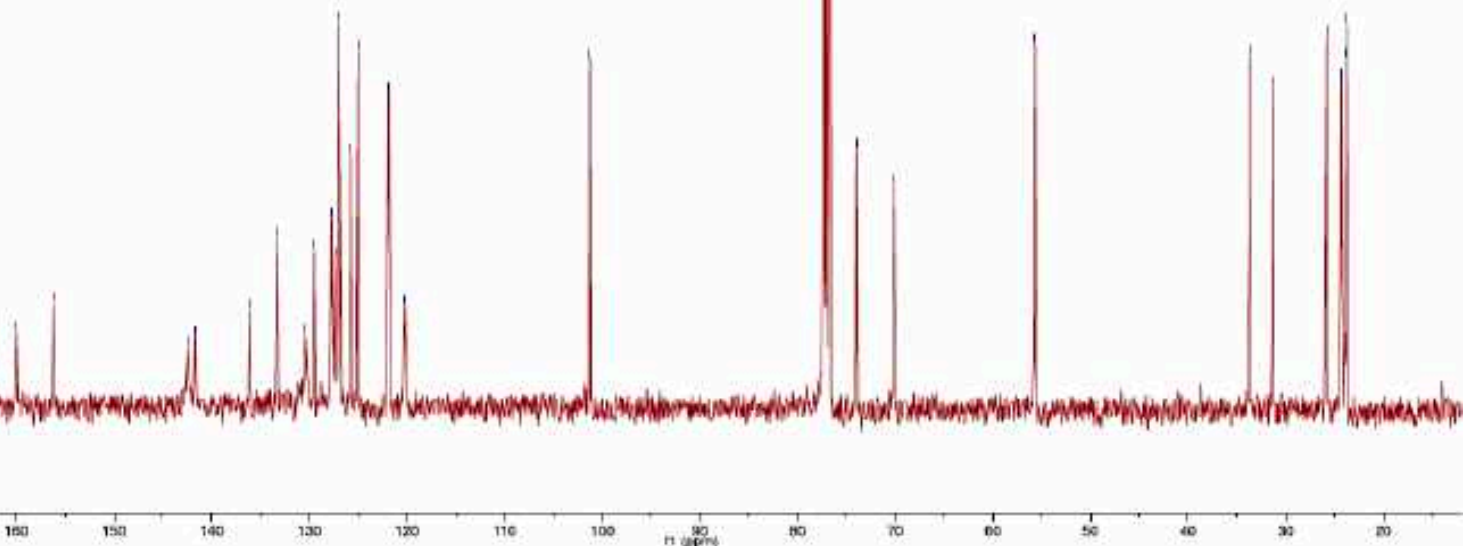
Racemic sample of $( \pm)$-9b: IA column, $n$-hexane:isopropanol 99:1, F= $1 \mathrm{~mL} / \mathrm{min}, \mathrm{T}=$ $30^{\circ} \mathrm{C}$.

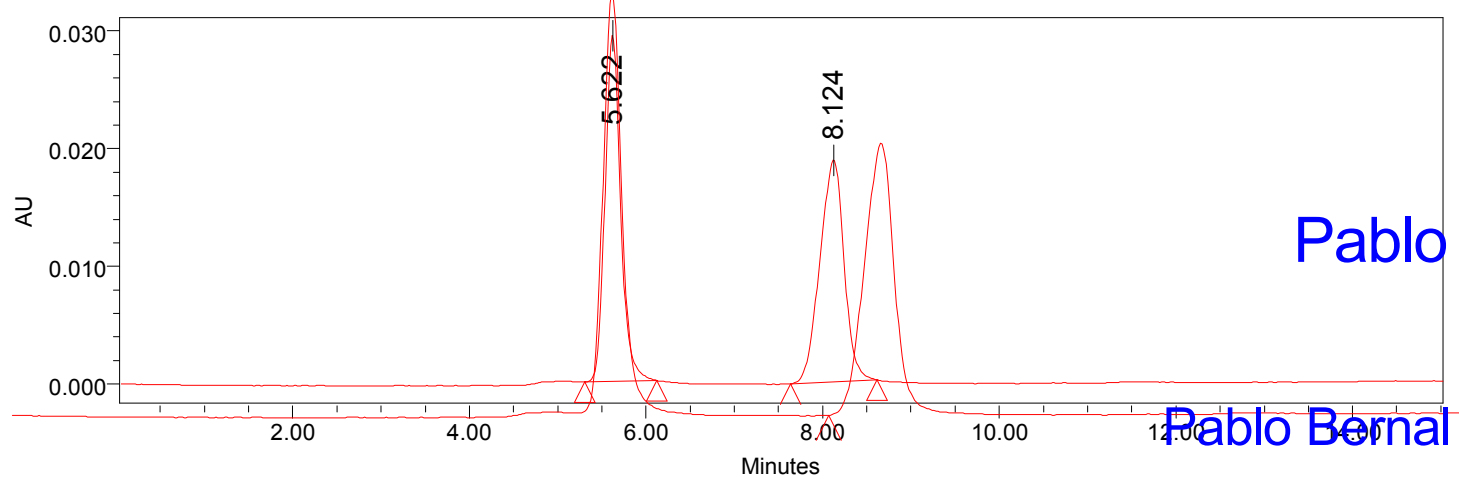

\section{Processed Channel: PDA 241.4 nm}

\begin{tabular}{|r|l|r|r|r|r|}
\hline & Processed Channel & $\begin{array}{r}\text { Retention } \\
\text { Time }(\min )\end{array}$ & Area & \% Area & Height \\
\hline 1 & PDA 241.4 nm & 5.622 & 362267 & 50.18 & 29407 \\
\hline 2 & PDA 241.4 nm & 8.124 & 359621 & 49.82 & 18878 \\
\hline
\end{tabular}

\section{Enantioriched sample of $\left(S_{a}, R\right)-9 \mathbf{b}$ :}

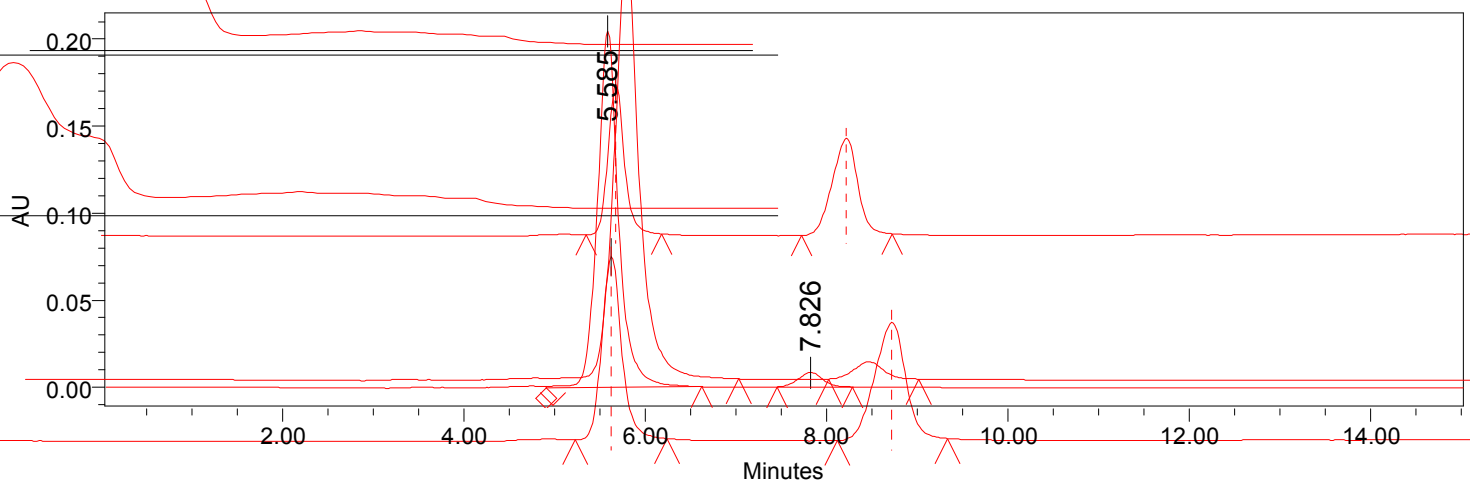

Processed Channel: PDA $239.2 \mathrm{~nm}$

\begin{tabular}{|r|l|r|r|r|r|}
\hline & Processed Channel & $\begin{array}{c}\text { Retention } \\
\text { Time }(\mathrm{min})\end{array}$ & Area & $\%$ Area & Height \\
\hline 1 & PDA 239.2 nm & 5.585 & 3344504 & 95.52 & 204640 \\
\hline 2 & PDA 239.2 nm & 7.826 & 156842 & 4.48 & 8339 \\
\hline
\end{tabular}


${ }^{1} \mathrm{H}$ NMR $\left(400 \mathrm{MHz}, \mathrm{CDCl}_{3}\right)$ of $\left(S_{a}, R\right)-\mathbf{9 c}$ :

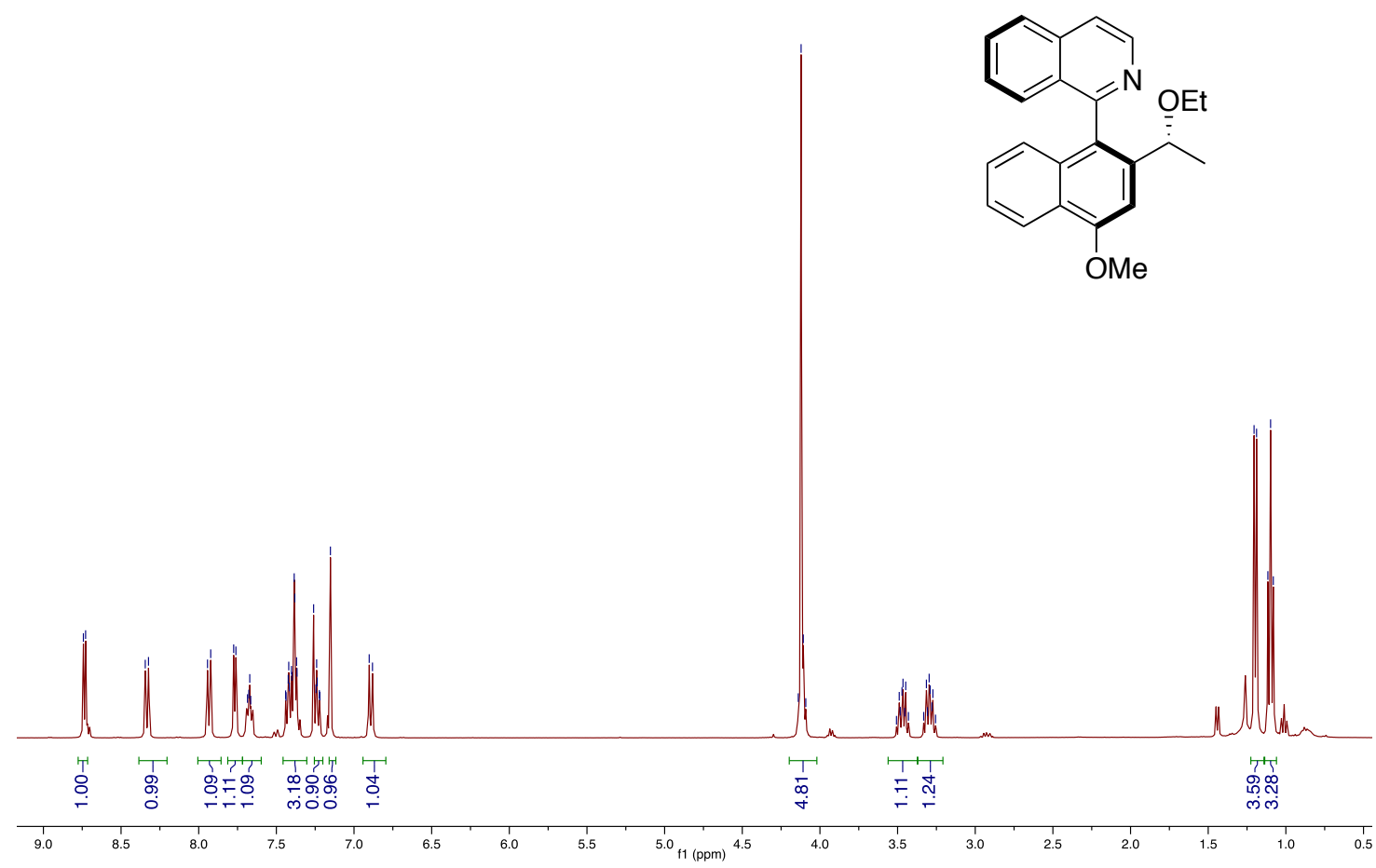

${ }^{13} \mathrm{C}$ NMR $\left(100 \mathrm{MHz}, \mathrm{CDCl}_{3}\right)$ of $\left(S_{a}, R\right)-\mathbf{9 c}$ :

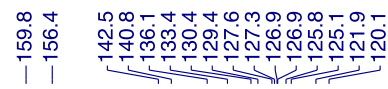

옹

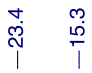

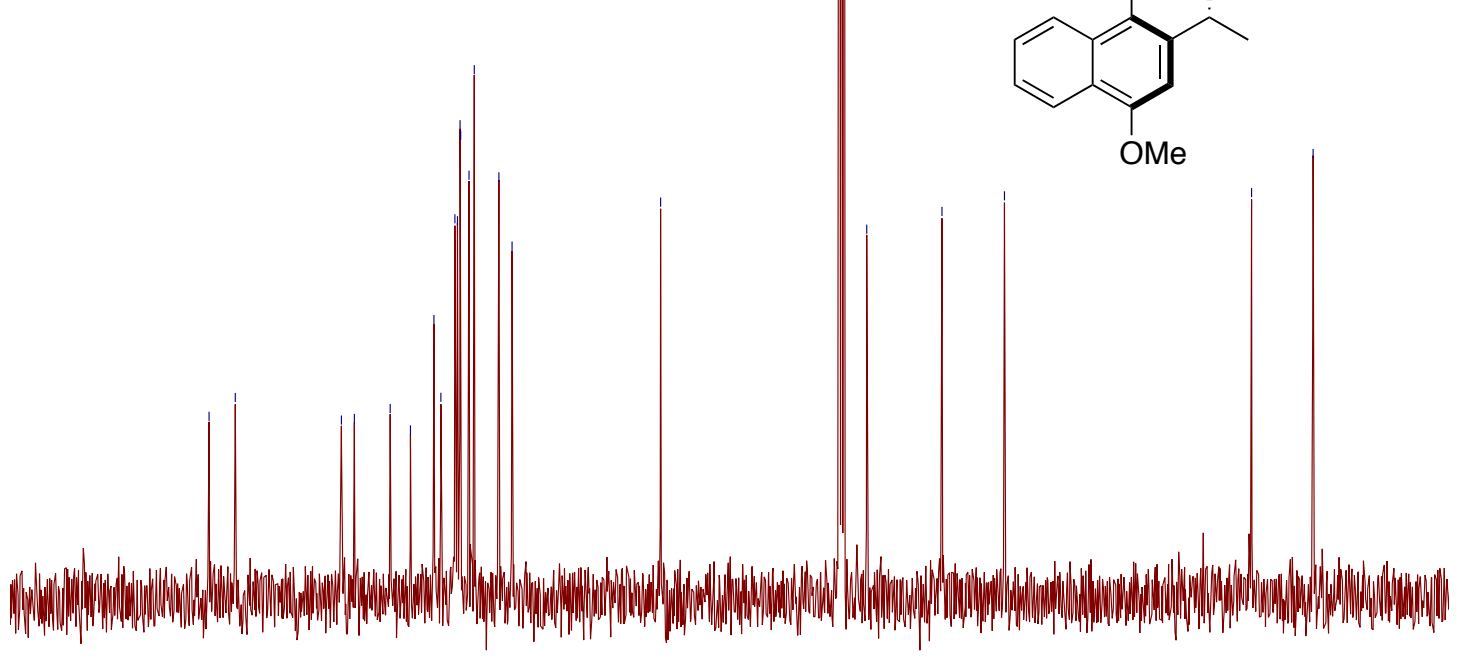<smiles>CCOC(C)c1cc(OC)c2ccccc2c1-c1nccc2ccccc12</smiles>

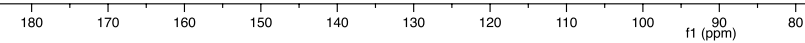


Racemic sample of $( \pm)$-9c: IA column, $n$-hexane:isopropanol 97:3, F= $1 \mathrm{~mL} / \mathrm{min}, \mathrm{T}=$ $30^{\circ} \mathrm{C}$.

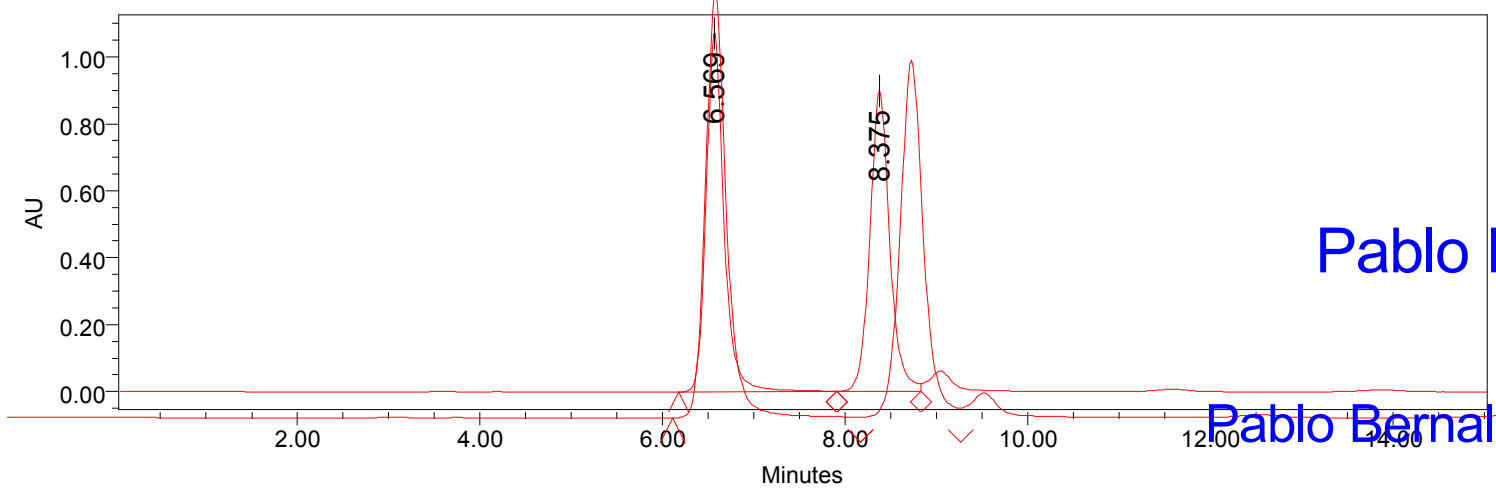

\section{Processed Channel: PDA $209.7 \mathrm{~nm}$}

\begin{tabular}{|r|l|r|r|r|r|}
\hline & Processed Channel & $\begin{array}{r}\text { Retention } \\
\text { Time }(\mathrm{min})\end{array}$ & Area & \% Area & Height \\
\hline 1 & PDA 209.7 nm & 6.569 & 14625710 & 51.90 & 1076402 \\
\hline 2 & PDA 209.7 nm & 8.375 & 13554438 & 48.10 & 899793 \\
\hline
\end{tabular}

Enantioriched sample of $\left(S_{a}, R\right)-9 \mathrm{c}$ :

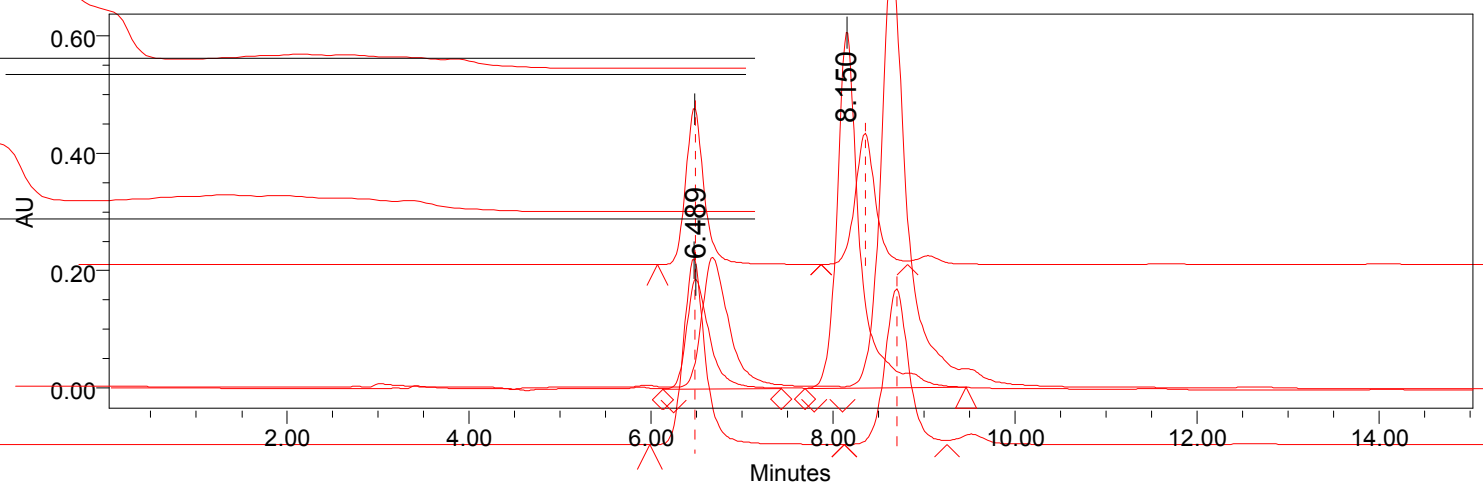

Processed Channel: PDA $217.8 \mathrm{~nm}$

\begin{tabular}{|r|l|r|r|r|r|}
\hline & Processed Channel & $\begin{array}{r}\text { Retention } \\
\text { Time }(\mathrm{min})\end{array}$ & Area & \% Area & Height \\
\hline 1 & PDA 217.8 nm & 6.489 & 3316224 & 23.60 & 187200 \\
\hline 2 & PDA 217.8 nm & 8.150 & 10736898 & 76.40 & 607884 \\
\hline
\end{tabular}


${ }^{1} \mathrm{H}$ NMR $\left(400 \mathrm{MHz}, \mathrm{CDCl}_{3}\right)$ of $\left(S_{a}, R\right)-\mathbf{9 d}$ :

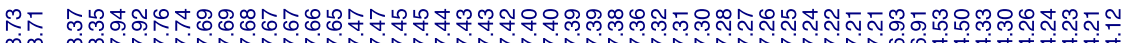

$\infty 0^{\infty}$

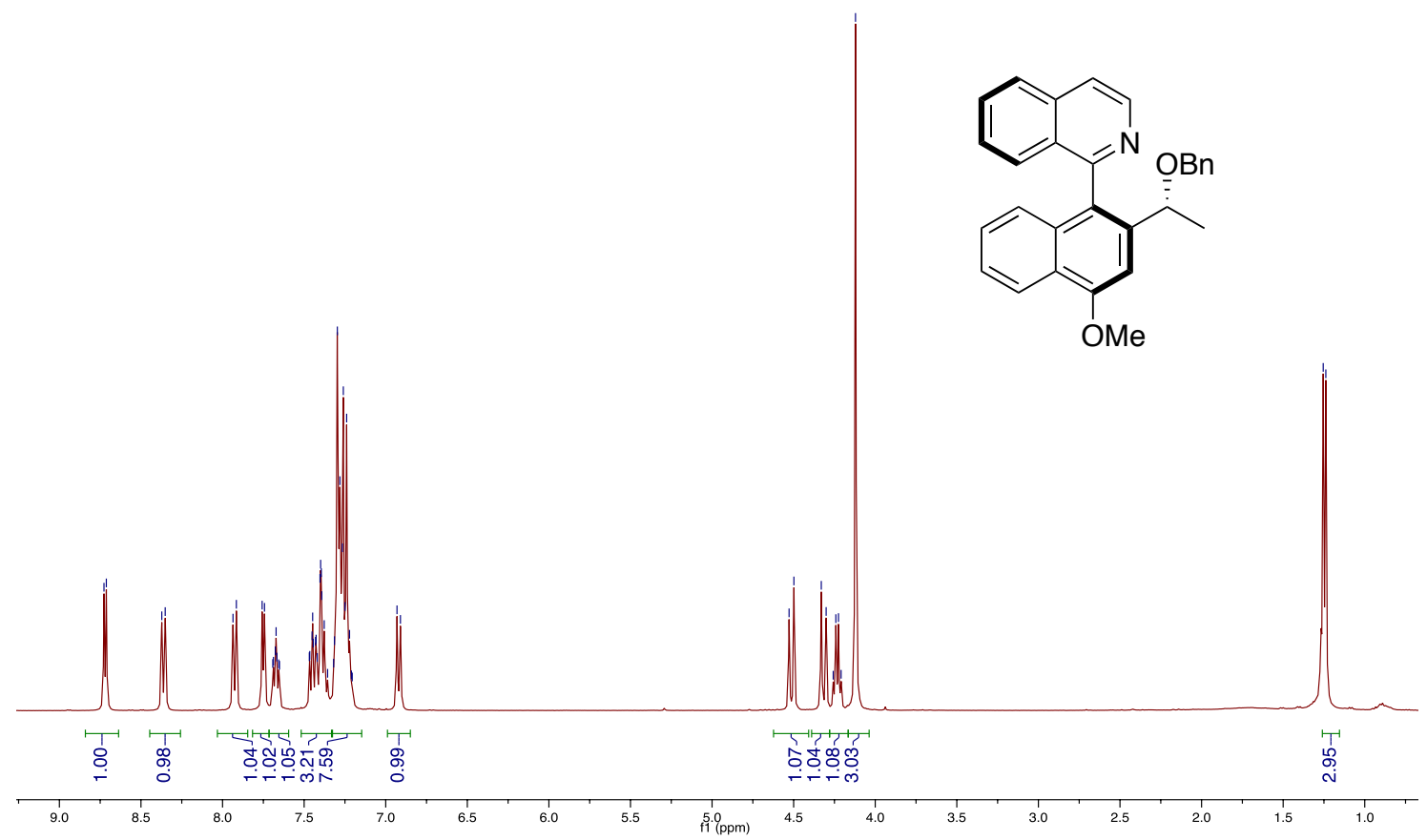

${ }^{13} \mathrm{C}$ NMR $\left(100 \mathrm{MHz}, \mathrm{CDCl}_{3}\right)$ of $\left(S_{a}, R\right)-\mathbf{9 d}$ :

人 إ

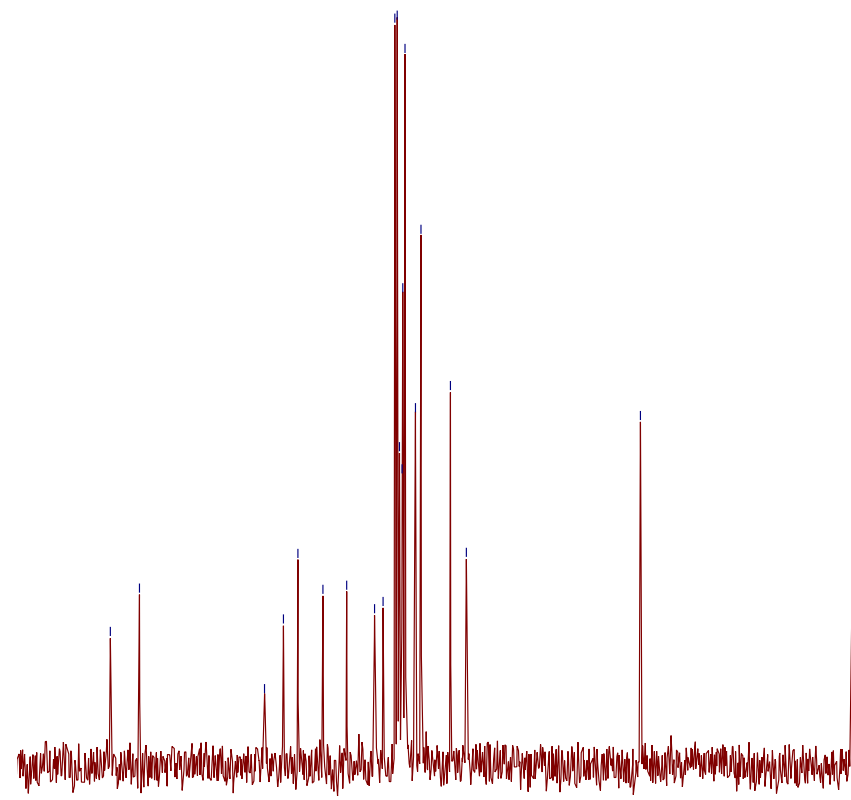<smiles>COc1cc(C(C)OCc2ccccc2)c(-c2nccc3ccccc23)c2ccccc12</smiles>

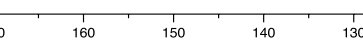


Racemic sample of $( \pm)$-9d: OD column, $n$-hexane:isopropanol 99:1, F= $1 \mathrm{~mL} / \mathrm{min}, \mathrm{T}=$ $30^{\circ} \mathrm{C}$.

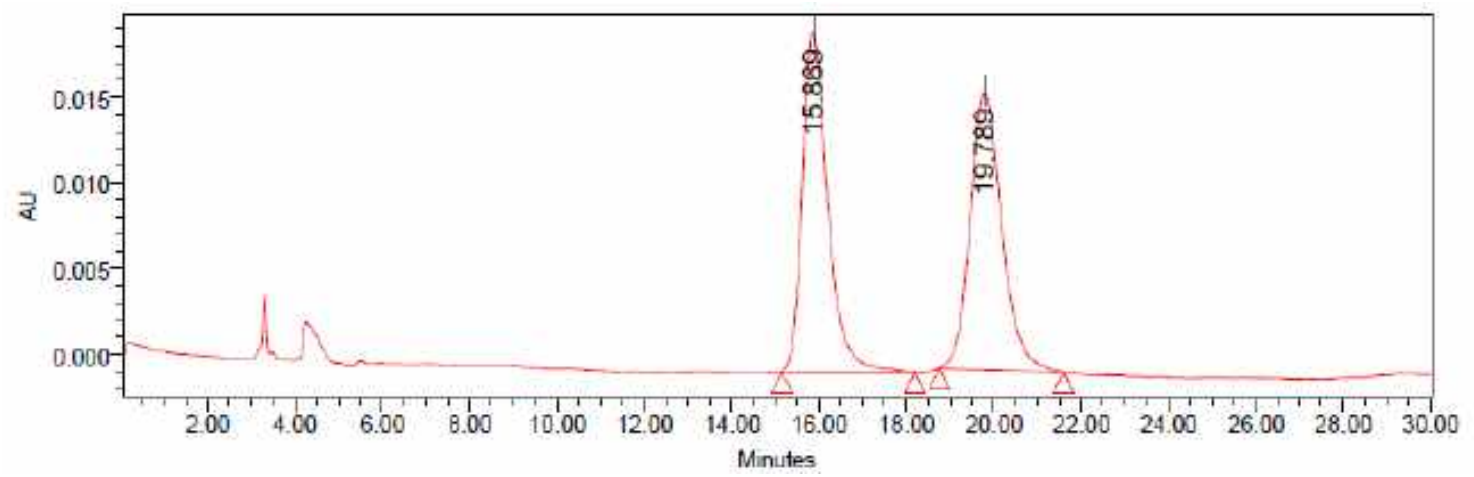

Processed Channel: PDA $220.0 \mathrm{~nm}$
\begin{tabular}{|l|l|r|r|r|r|}
\hline & Processed Channel & $\begin{array}{c}\text { Retention } \\
\text { Time (min) }\end{array}$ & Area & $\%$ Area & Height \\
\hline 1 & PDA $220.0 \mathrm{~nm}$ & 15.869 & 801413 & 49.62 & 19891 \\
\hline 2 & PDA $220.0 \mathrm{~nm}$ & 19.789 & 813646 & 50.38 & 16231 \\
\hline
\end{tabular}

Enantioriched sample of $\left(S_{a}, R\right)$ - 9d:

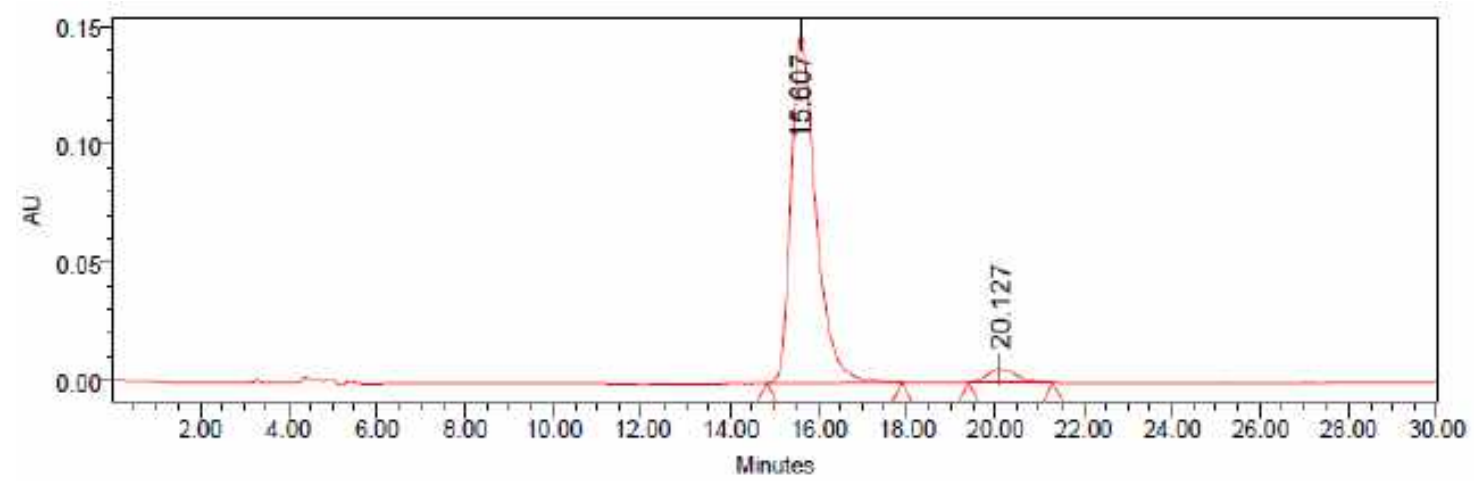

Processed Channel: PDA $220.0 \mathrm{~nm}$

\begin{tabular}{|r|l|r|r|r|r|}
\hline & Processed Channel & $\begin{array}{l}\text { Retention } \\
\text { Time }(\mathrm{min})\end{array}$ & Area & $\%$ Area & Height \\
\hline 1 & PDA $220.0 \mathrm{~nm}$ & 15.607 & 5702235 & 95.53 & 147390 \\
\hline 2 & PDA $220.0 \mathrm{~nm}$ & 20.127 & 266676 & 4.47 & 5528 \\
\hline
\end{tabular}


${ }^{1} \mathrm{H}$ NMR $\left(400 \mathrm{MHz}, \mathrm{CDCl}_{3}\right)$ of $\left(S_{a}, R\right)-\mathbf{9 e}:$

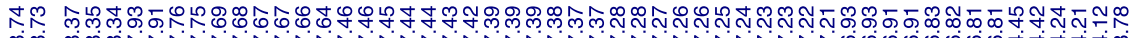

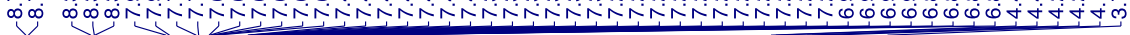

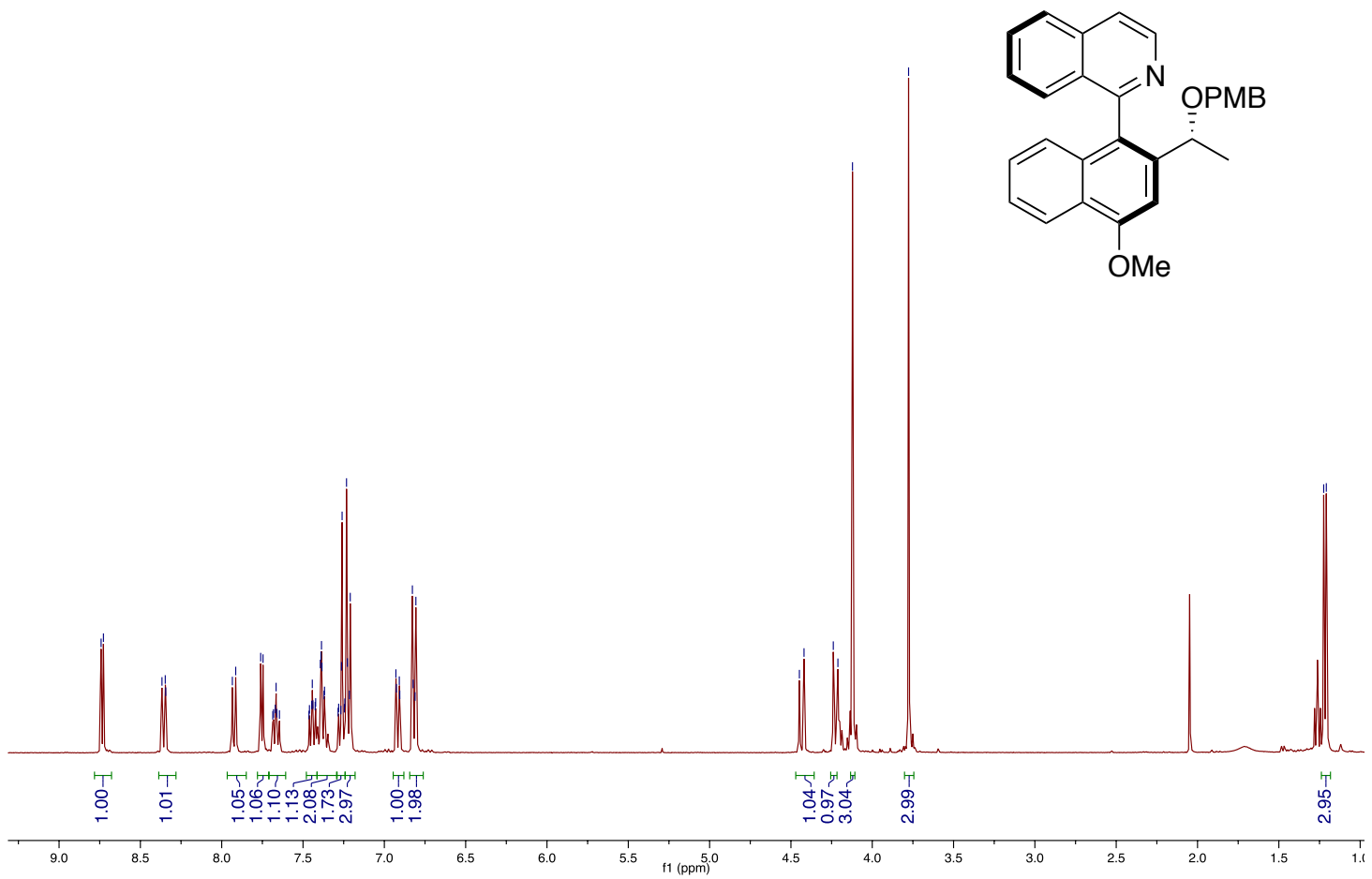

${ }^{13} \mathrm{C}$ NMR $\left(100 \mathrm{MHz}, \mathrm{CDCl}_{3}\right)$ of $\left(S_{a}, R\right)-\mathbf{9 e}$ :

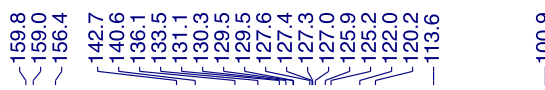

$\stackrel{9}{\circ}$

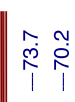

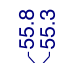

$\stackrel{\llcorner}{\stackrel{\infty}{j}}$

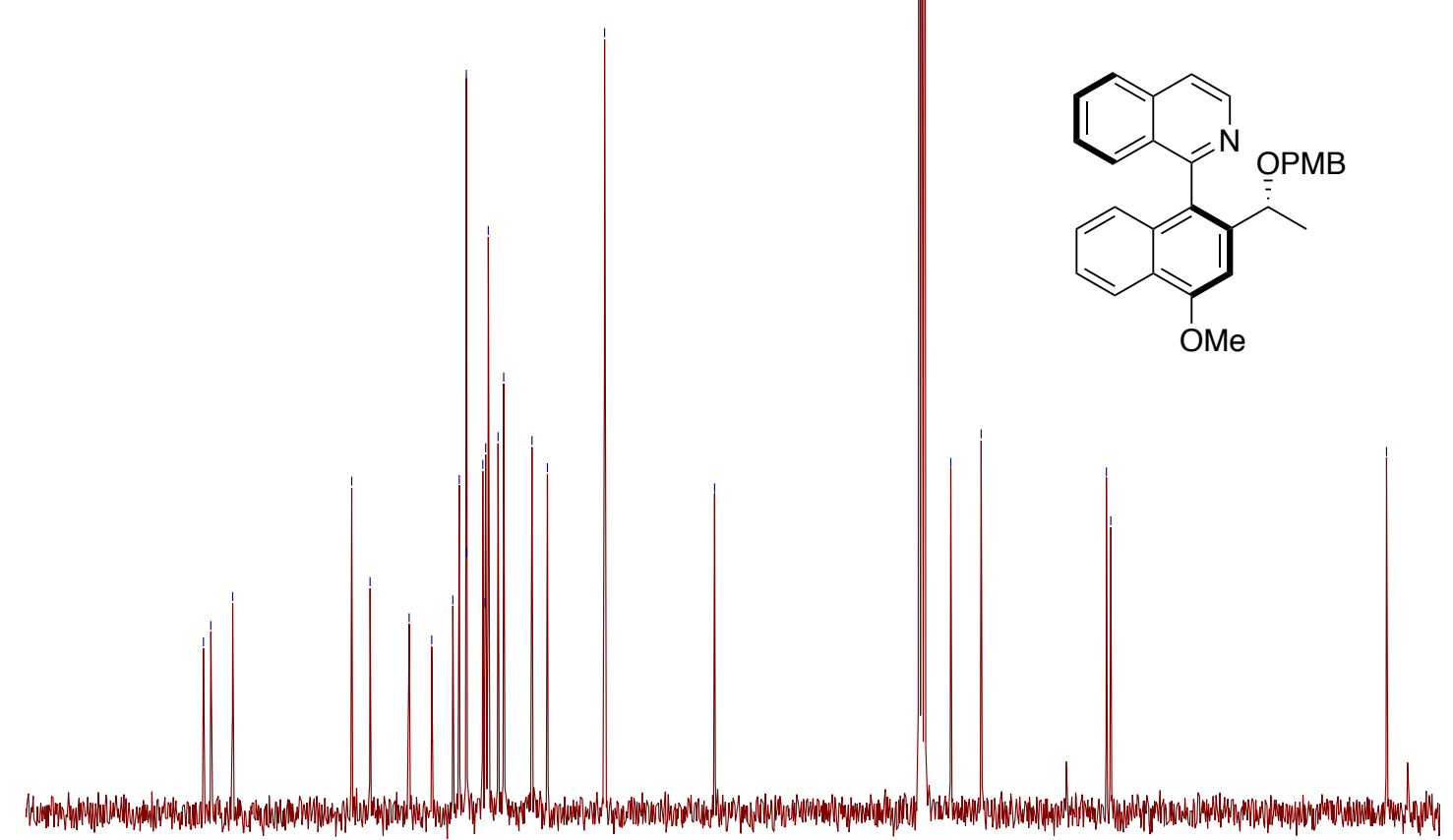

${ }_{80}$

160

140 
Racemic sample of $( \pm)$-9e: OD column, $n$-hexane:isopropanol 95:5, F= $1 \mathrm{~mL} / \mathrm{min}, \mathrm{T}=$ $30^{\circ} \mathrm{C}$.

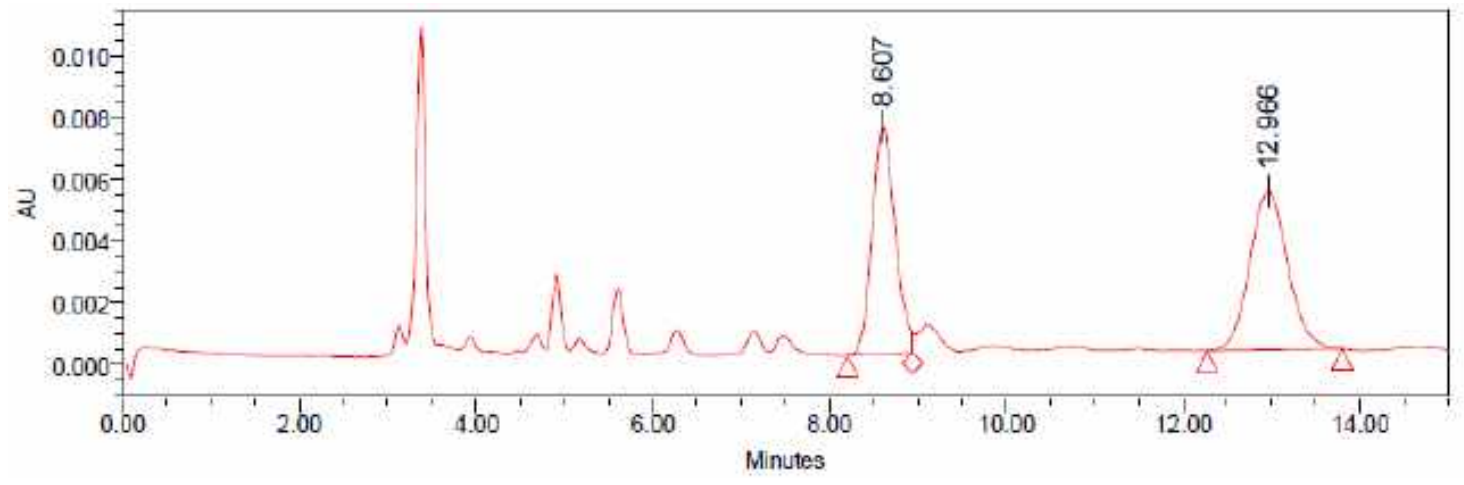

Processed Channel: PDA $232.3 \mathrm{~nm}$
\begin{tabular}{|l|l|r|r|r|r|}
\hline & Processed Channel & $\begin{array}{l}\text { Retention } \\
\text { Time (min) }\end{array}$ & Area & $\%$ Area & Height \\
\hline 1 & PDA $232.3 \mathrm{~nm}$ & 8.607 & 133627 & 46.65 & 7374 \\
\hline 2 & PDA $232.3 \mathrm{~nm}$ & 12.966 & 152808 & 53.35 & 5161 \\
\hline
\end{tabular}

Enantioriched sample of $\left(S_{a}, R\right)$ - 9e:

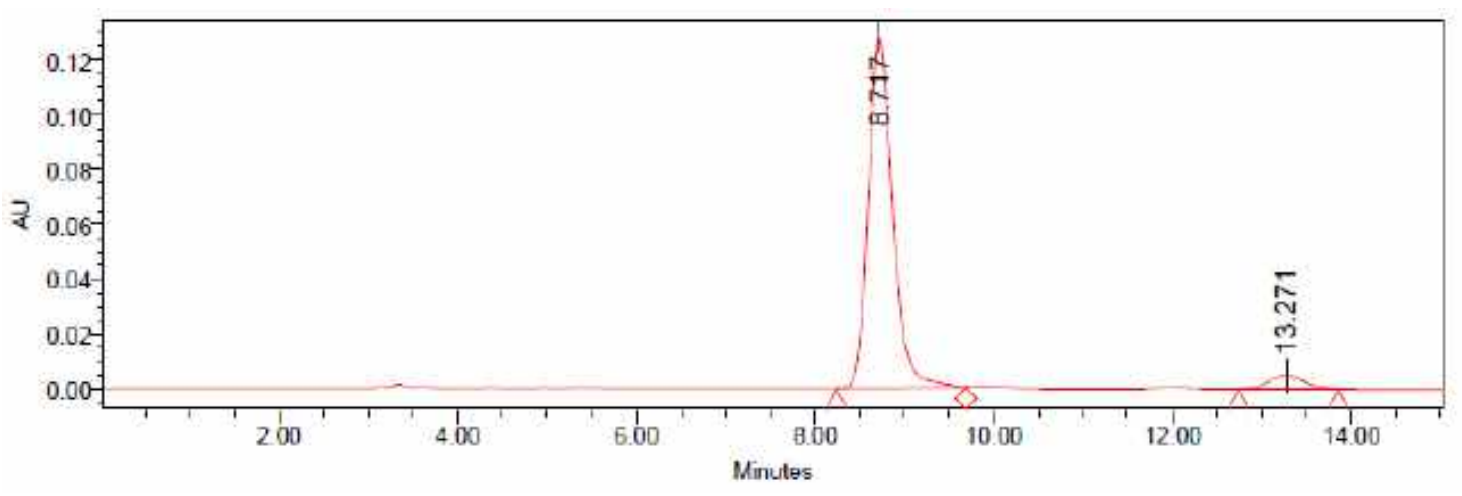

Processed Channel: PDA $232.3 \mathrm{~nm}$

\begin{tabular}{|l|l|r|r|r|r|}
\hline & Processed Channel & $\begin{array}{c}\text { Retention } \\
\text { Time }(\mathrm{min})\end{array}$ & Area & $\%$ Area & Height \\
\hline 1 & PDA $232.3 \mathrm{~nm}$ & 8.717 & 2424997 & 94.38 & 128378 \\
\hline 2 & PDA $232.3 \mathrm{~nm}$ & 13.271 & 144300 & 5.62 & 5082 \\
\hline
\end{tabular}


${ }^{1} \mathrm{H}$ NMR $\left(400 \mathrm{MHz}, \mathrm{CDCl}_{3}\right)$ of $\left(S_{a}, R\right)-9 f:$

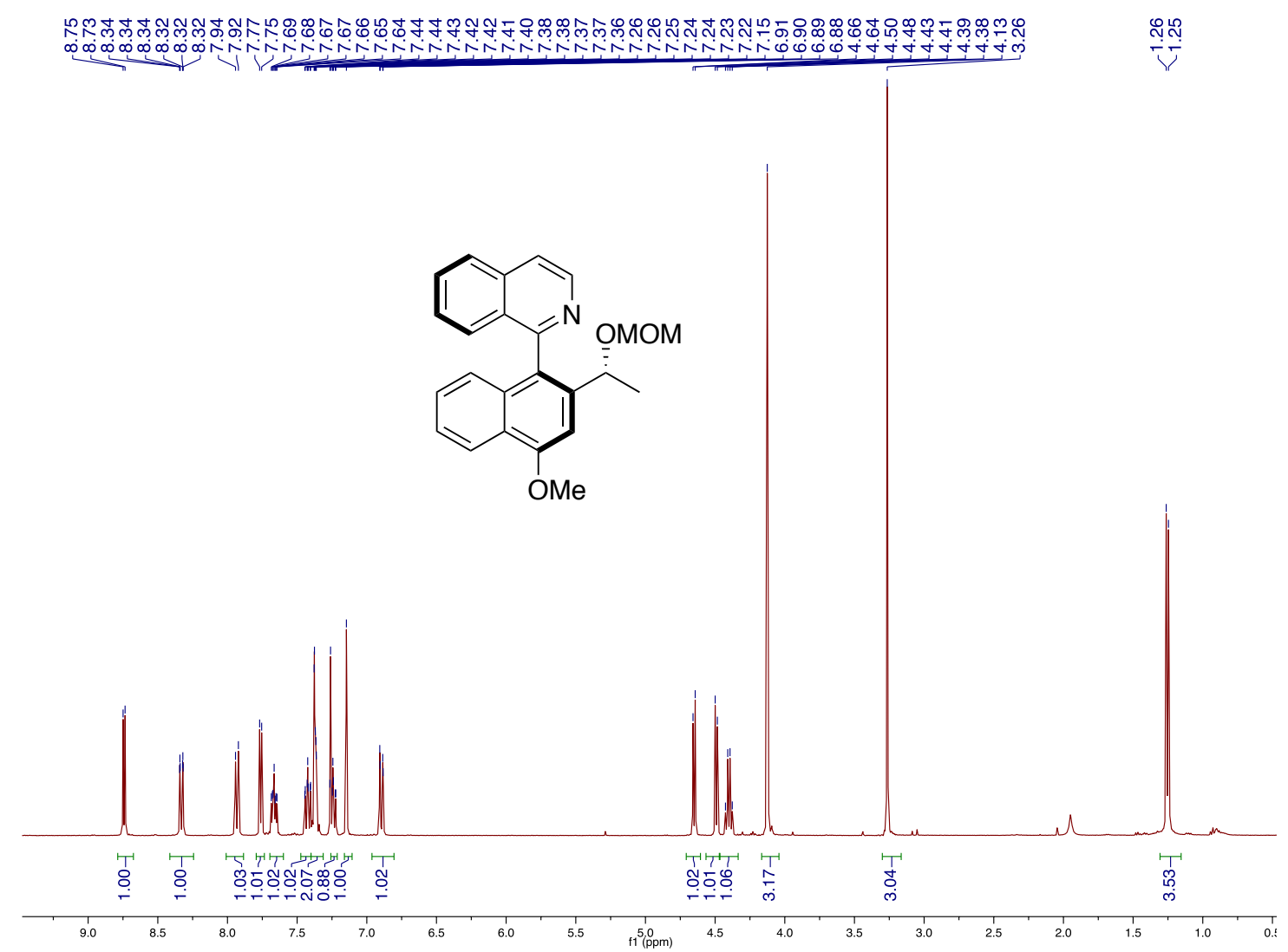

${ }^{13} \mathrm{C}$ NMR $\left(100 \mathrm{MHz}, \mathrm{CDCl}_{3}\right)$ of $\left(S_{a}, R\right)$-9f:

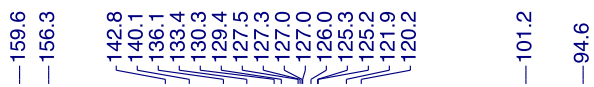

ז

$\stackrel{\circ}{\text { N }}$
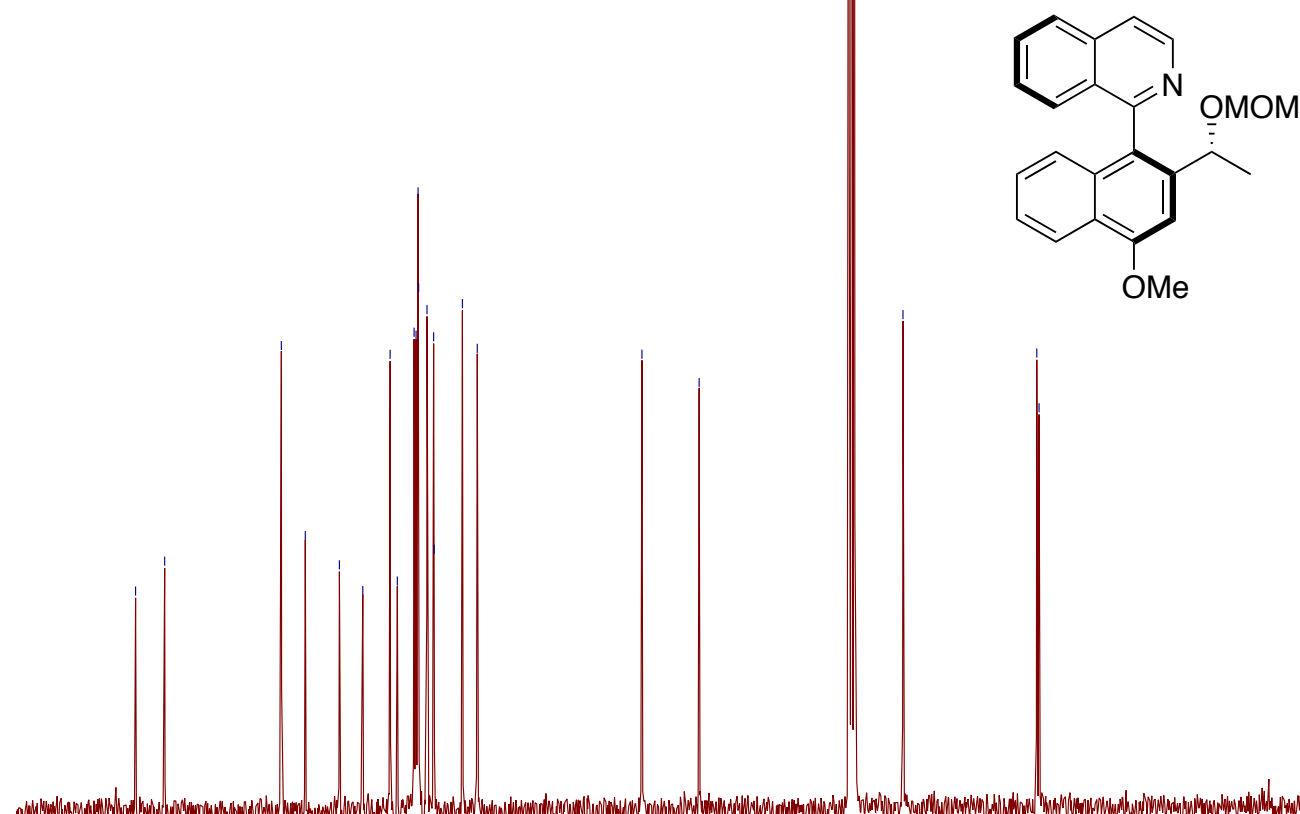

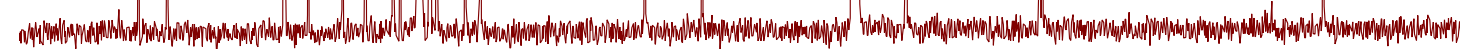

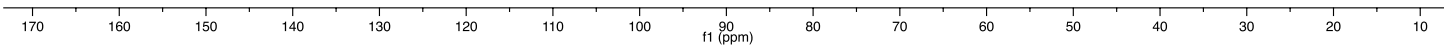


Racemic sample of $( \pm)$-9f: IA column, $n$-hexane:isopropanol 95:5, F= $1 \mathrm{~mL} / \mathrm{min}, \mathrm{T}=$ $30^{\circ} \mathrm{C}$.

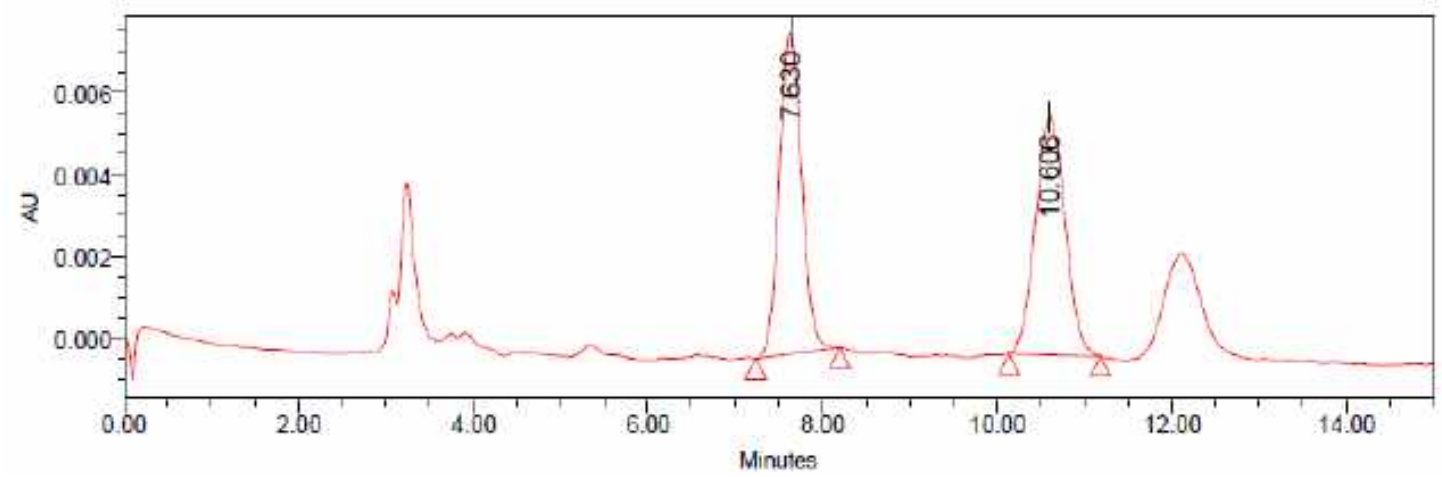

\begin{tabular}{|c|c|c|c|c|c|}
\hline & Processed Channel & $\begin{array}{l}\text { Retention } \\
\text { Time (min) }\end{array}$ & Area & $\%$ Area & Height \\
\hline 1 & PDA $220.0 \mathrm{~nm}$ & 7.630 & 138758 & 50.26 & 7826 \\
\hline 2 & PDA $220.0 \mathrm{~nm}$ & 10.606 & 137306 & 49.74 & 5808 \\
\hline
\end{tabular}

Enantioriched sample of $\left(S_{a}, R\right)-9 f$ :

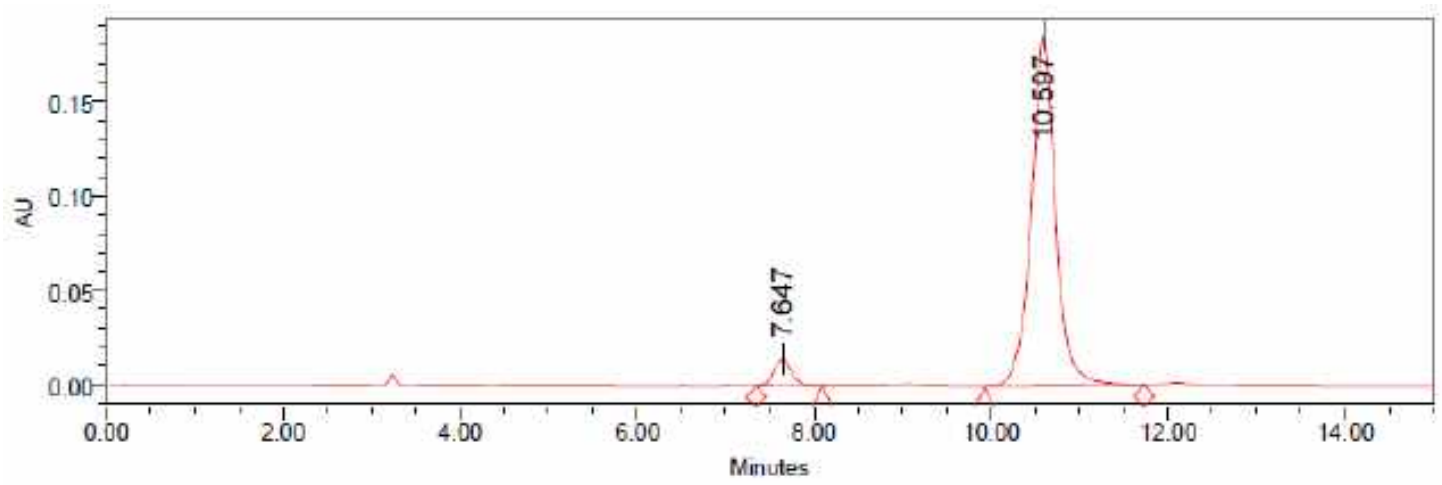

\begin{tabular}{c|} 
Processed Channel: PDA 220.0 nm \\
\hline
\end{tabular}


${ }^{1} \mathrm{H}$ NMR (400 MHz, $\mathrm{CDCl}_{3}$ ) of the minor diastereomer of $\mathbf{9 g}$ :

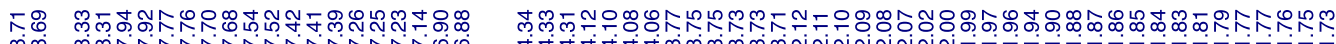

i)<smiles>COc1cc(C2CCCO2)c(-c2nccc3ccccc23)c2ccccc12</smiles>

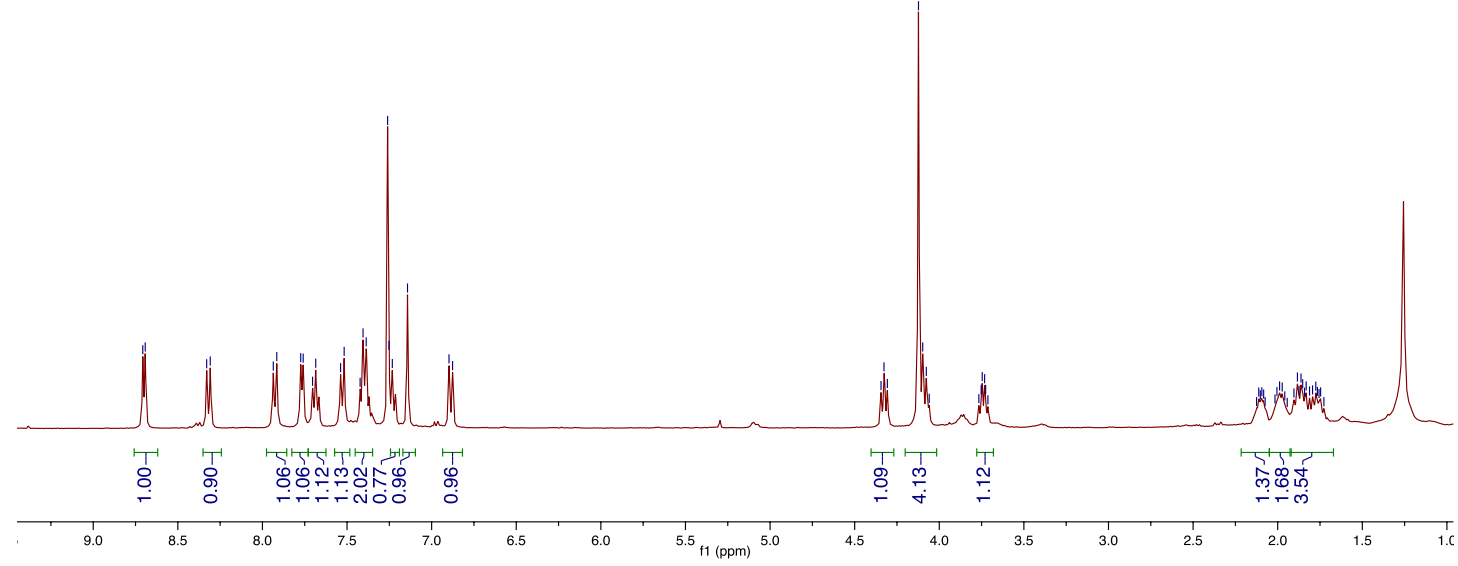

${ }^{13} \mathrm{C}$ NMR $\left(100 \mathrm{MHz}, \mathrm{CDCl}_{3}\right.$ ) of the minor diastereomer of $\mathbf{8 g}$ :

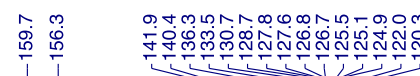

웅우

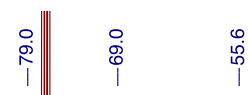

অ্户<smiles>COc1cc(C2CCCO2)c(-c2nccc3ccccc23)c2ccccc12</smiles>

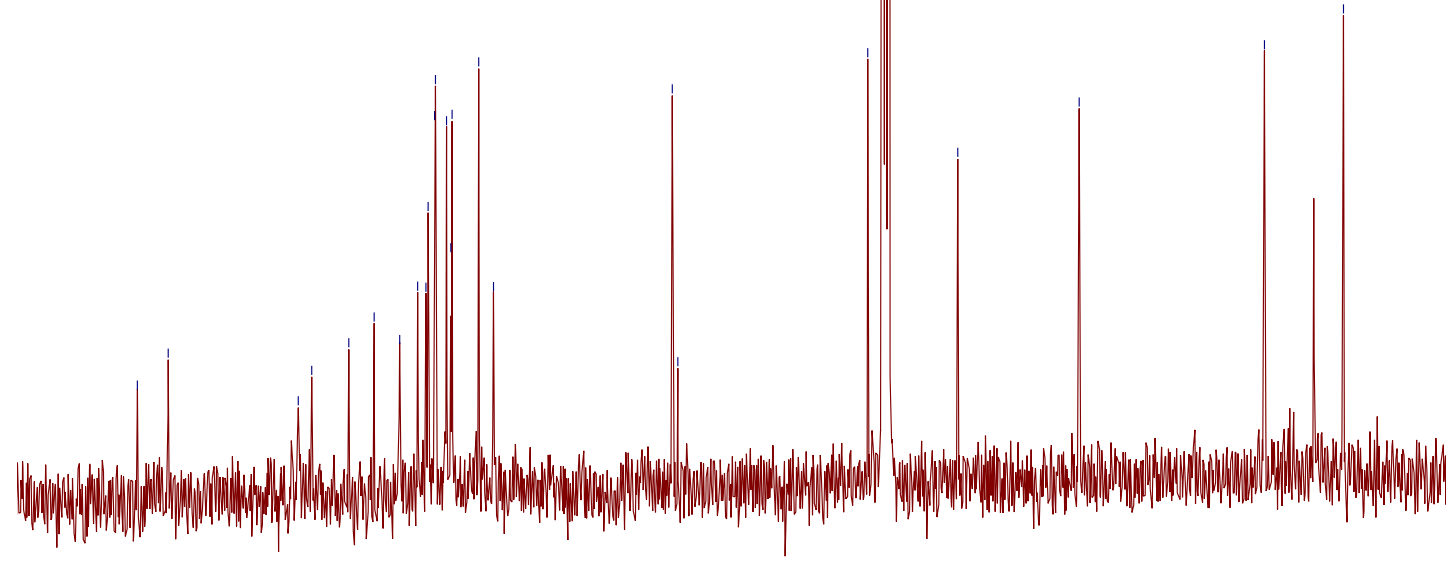


Racemic sample of the minor diastereomer of $9 \mathrm{~g}$ : IA column, $n$-hexane:isopropanol 97:, $\mathrm{F}=1 \mathrm{~mL} / \mathrm{min}, \mathrm{T}=30^{\circ} \mathrm{C}$.

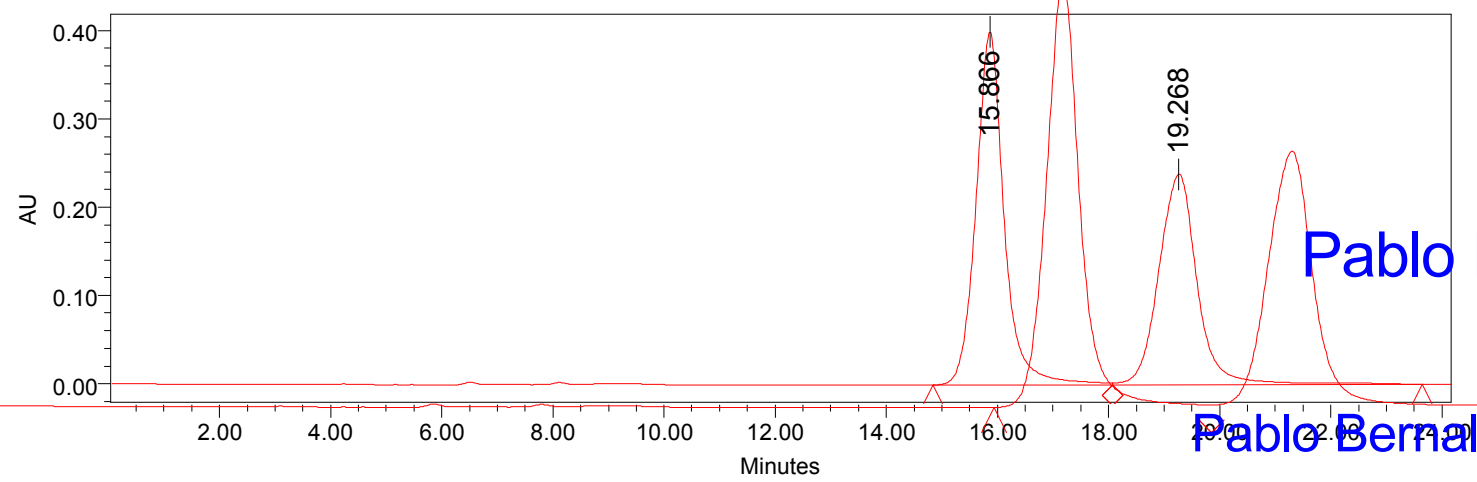

Processed Channel: PDA $216.0 \mathrm{~nm}$

\begin{tabular}{|r|l|r|r|r|r|}
\hline & Processed Channel & $\begin{array}{c}\text { Retention } \\
\text { Time (min) }\end{array}$ & Area & $\%$ Area & Height \\
\hline 1 & PDA $216.0 \mathrm{~nm}$ & 15.866 & 14053975 & 55.16 & 400016 \\
\hline 2 & PDA $216.0 \mathrm{~nm}$ & 19.268 & 11424879 & 44.84 & 238265 \\
\hline
\end{tabular}

\section{Enantioriched sample of the minor diastereomer of $9 \mathrm{~g}$ :}

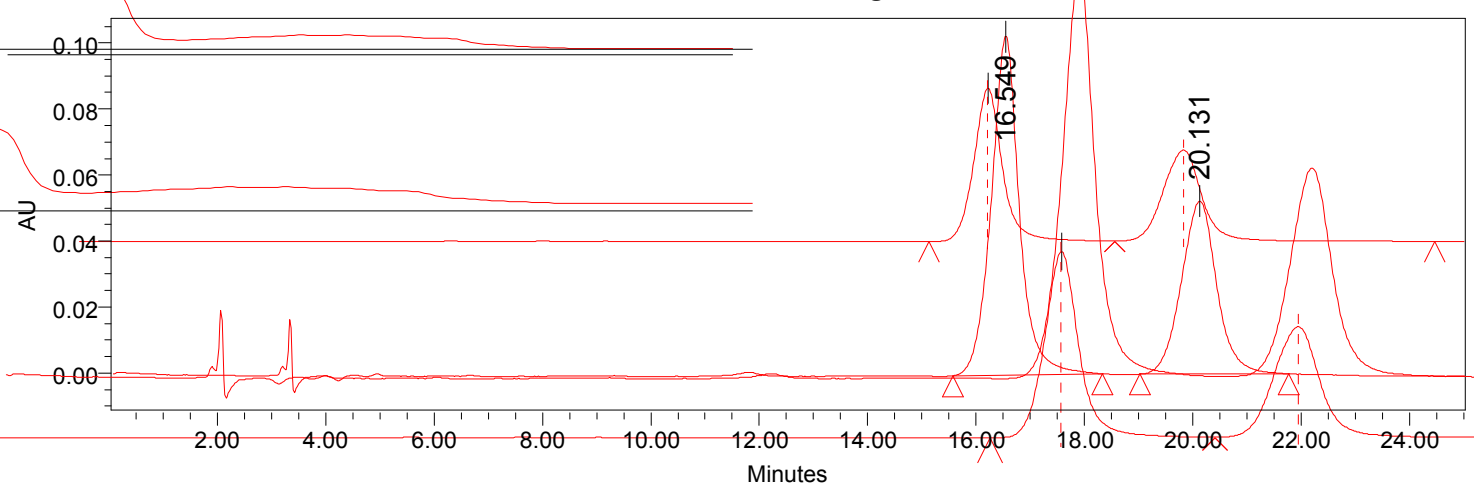

Processed Channel: PDA 214.3 nm

\begin{tabular}{|r|l|r|c|r|r|}
\hline & Processed Channel & $\begin{array}{c}\text { Retention } \\
\text { Time (min) }\end{array}$ & Area & \% Area & Height \\
\hline 1 & PDA 214.3 nm & 16.549 & 3726313 & 61.07 & 102673 \\
\hline 2 & PDA 214.3 nm & 20.131 & 2375264 & 38.93 & 52373 \\
\hline
\end{tabular}


${ }^{1} \mathrm{H} \mathrm{NMR}\left(400 \mathrm{MHz}, \mathrm{CDCl}_{3}\right)$ of $\left(R_{a}, S\right)-9 \mathrm{~g}$ :

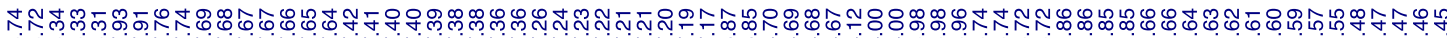

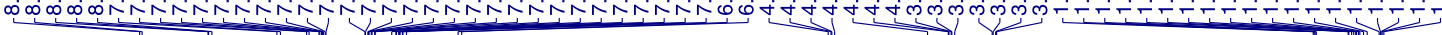<smiles>COc1cc([C@H]2CCCO2)c(-c2nccc3ccccc23)c2ccccc12</smiles>

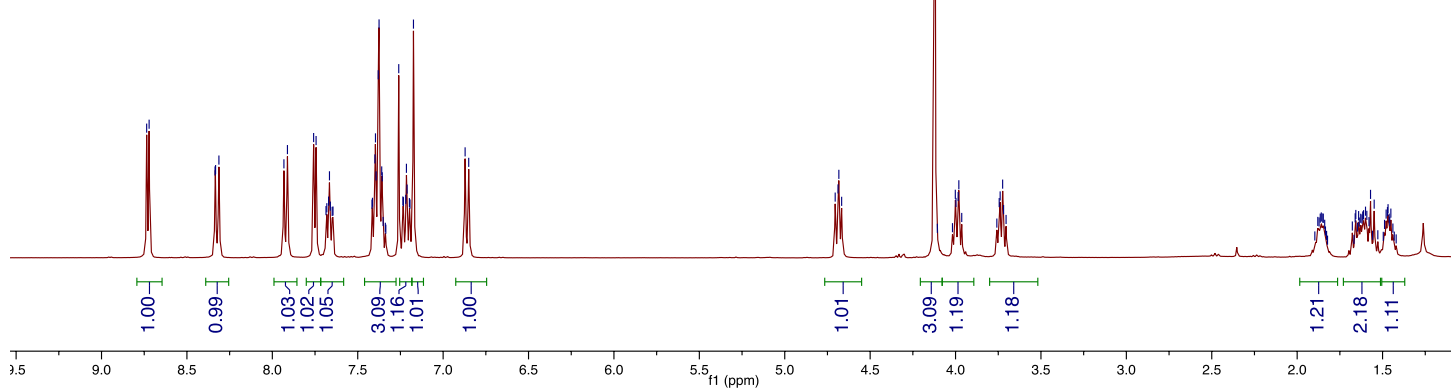

${ }^{13} \mathrm{C}$ NMR $\left(100 \mathrm{MHz}, \mathrm{CDCl}_{3}\right)$ of $\left(R_{a}, S\right)-\mathbf{9 g}$ :

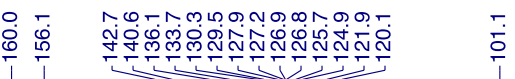

高<smiles>COc1cc([C@H]2CCCO2)c(-c2nccc3ccccc23)c2ccccc12</smiles>

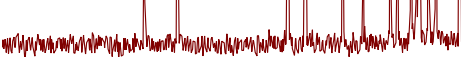
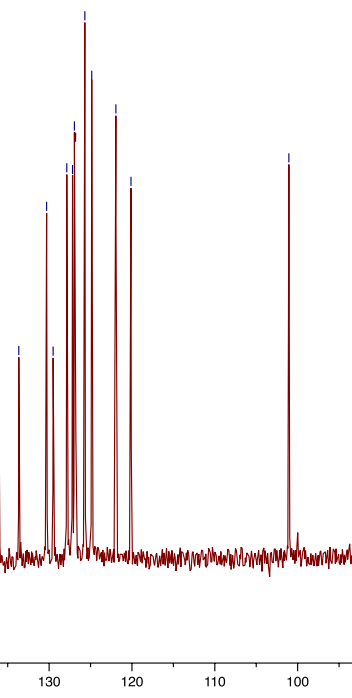
Racemic sample of the $( \pm)$-major diastereomer of $9 \mathrm{~g}$ : IA column, $n$ hexane:isopropanol 90:10, $\mathrm{F}=1 \mathrm{~mL} / \mathrm{min}, \mathrm{T}=30^{\circ} \mathrm{C}$.

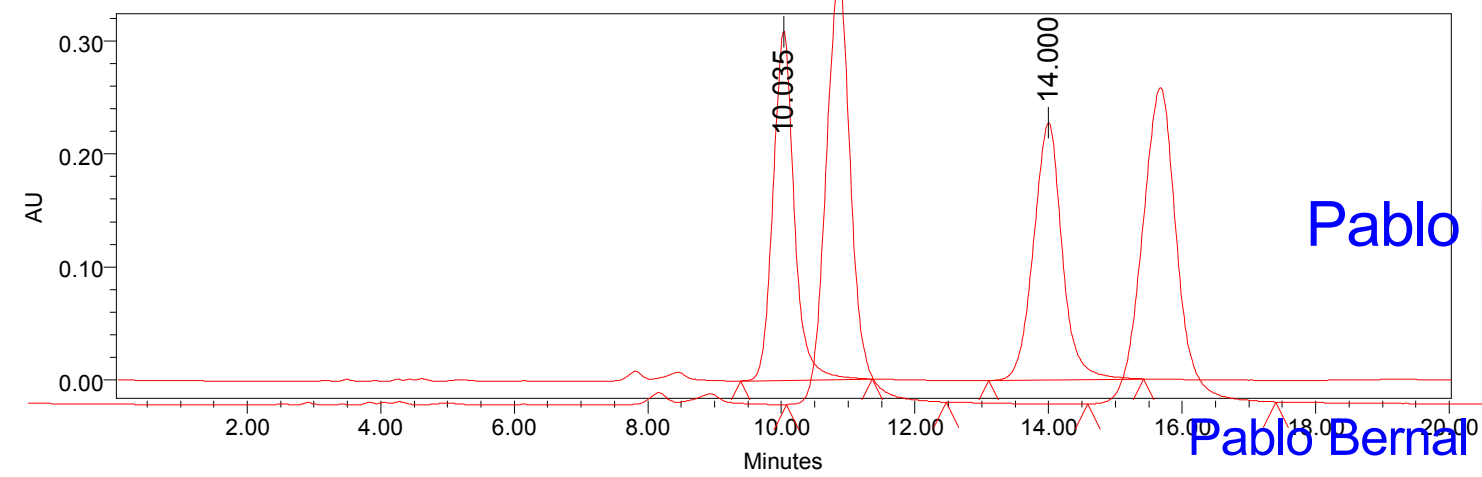

Processed Channel: PDA 218.0 nm

\begin{tabular}{|r|l|r|c|r|r|}
\hline & Processed Channel & $\begin{array}{c}\text { Retention } \\
\text { Time }(\mathrm{min})\end{array}$ & Area & $\%$ Area & Height \\
\hline 1 & PDA $218.0 \mathrm{~nm}$ & 10.035 & 6781278 & 50.07 & 309270 \\
\hline 2 & PDA $218.0 \mathrm{~nm}$ & 14.000 & 6762490 & 49.93 & 227595 \\
\hline
\end{tabular}

\section{Enantioriched sample of $\left(R_{a}, S\right)-9 \mathrm{~g}$ :}

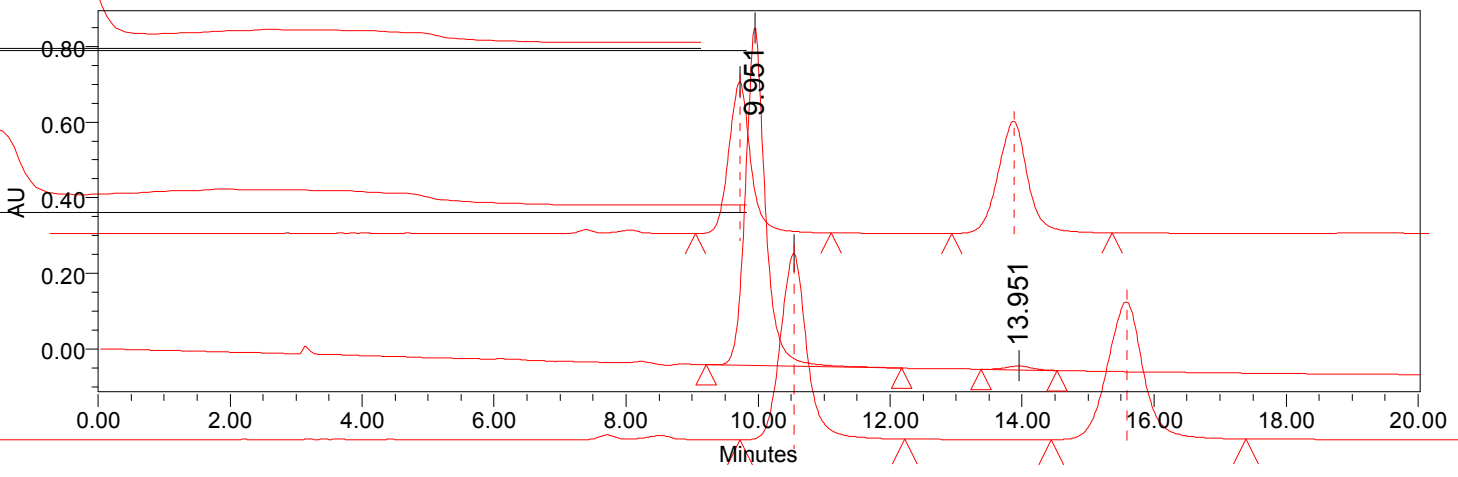

Processed Channel: PDA $232.5 \mathrm{~nm}$

\begin{tabular}{|r|l|r|r|r|r|}
\hline & Processed Channel & $\begin{array}{r}\text { Retention } \\
\text { Time }(\mathrm{min})\end{array}$ & Area & \% Area & Height \\
\hline 1 & PDA $232.5 \mathrm{~nm}$ & 9.951 & 18580208 & 98.56 & 893988 \\
\hline 2 & PDA $232.5 \mathrm{~nm}$ & 13.951 & 270997 & 1.44 & 10285 \\
\hline
\end{tabular}


${ }^{1} \mathrm{H}$ NMR $\left(400 \mathrm{MHz}, \mathrm{CDCl}_{3}\right)$ of $\left(S_{a}, R\right)-\mathbf{1 0 a}$ :

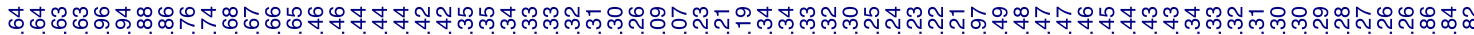

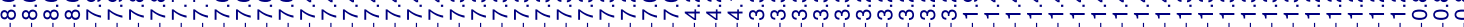<smiles>CCCCOC(C)c1ccc2ccccc2c1-c1ncccc1C</smiles>

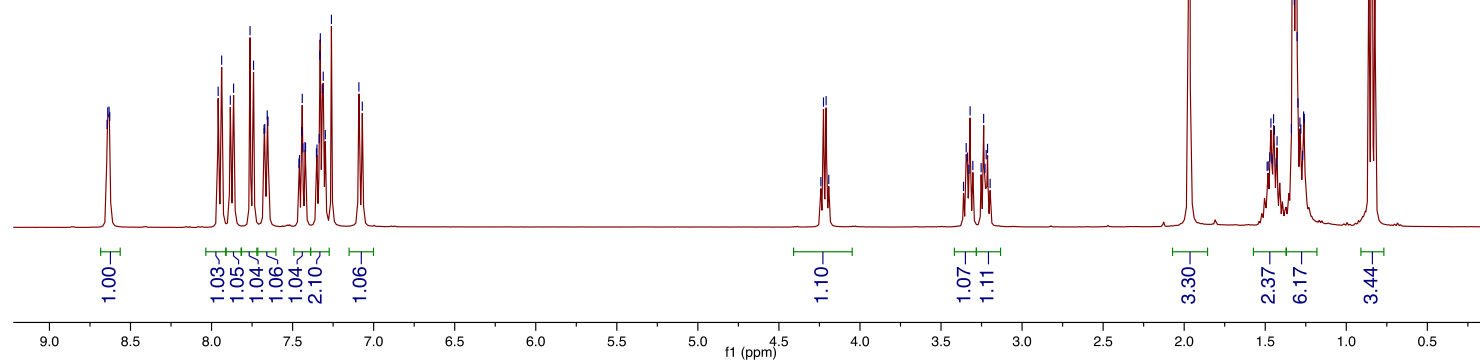

${ }^{13} \mathrm{C}$ NMR $\left(100 \mathrm{MHz}, \mathrm{CDCl}_{3}\right)$ of $\left(S_{a} R\right)-\mathbf{1 0 a}$ :

؟ุ.

芦

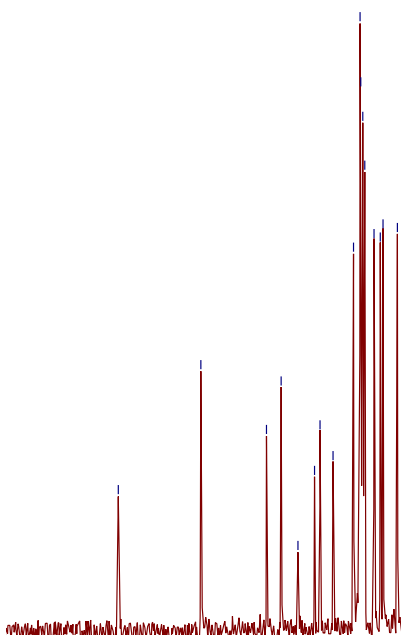<smiles>CCCCOC(C)c1ccc2ccccc2c1-c1ncccc1C</smiles>

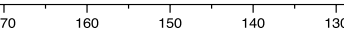

120

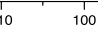


Racemic sample of $( \pm)$-10a: IA column, $n$-hexane:isopropanol 97:3, F= $1 \mathrm{~mL} / \mathrm{min}, \mathrm{T}=$ $30^{\circ} \mathrm{C}$.

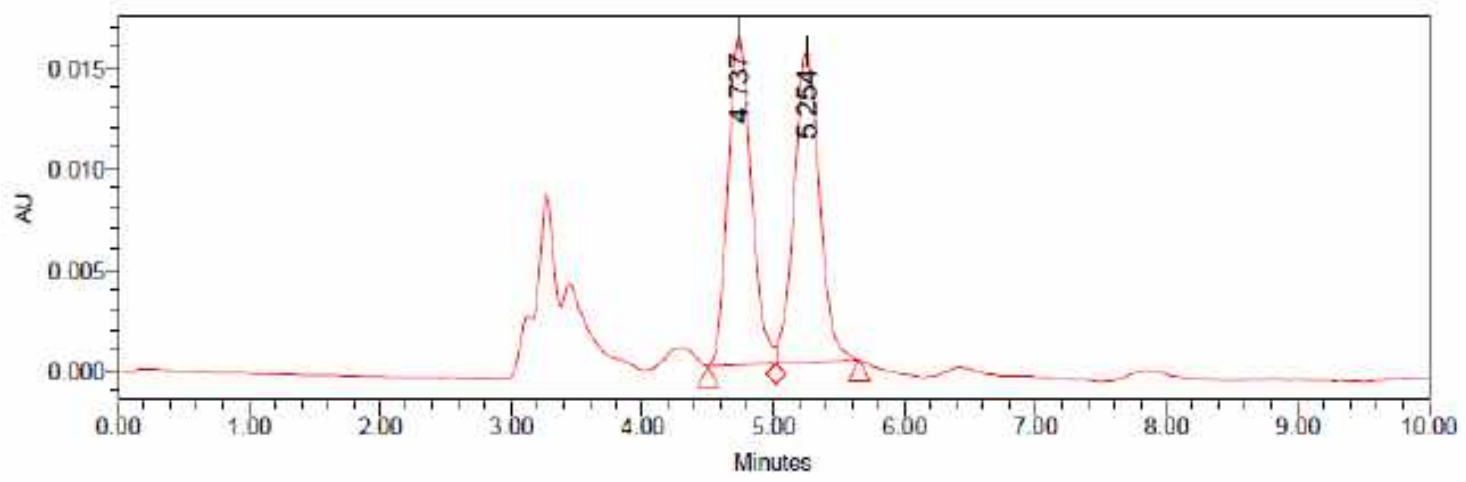

Processed Channel: PDA $225.0 \mathrm{~nm}$

\begin{tabular}{|l|l|r|r|r|r|}
\hline & Processed Channel & $\begin{array}{r}\text { Retention } \\
\text { Time (min) }\end{array}$ & Area & \% Area & Height \\
\hline 1 & PDA 225.0 nm & 4.737 & 204842 & 49.81 & 16399 \\
\hline 2 & PDA 225.0 nm & 5.254 & 206383 & 50.19 & 15416 \\
\hline
\end{tabular}

Enantioriched sample of $\left(S_{a}, R\right)-10 a:$

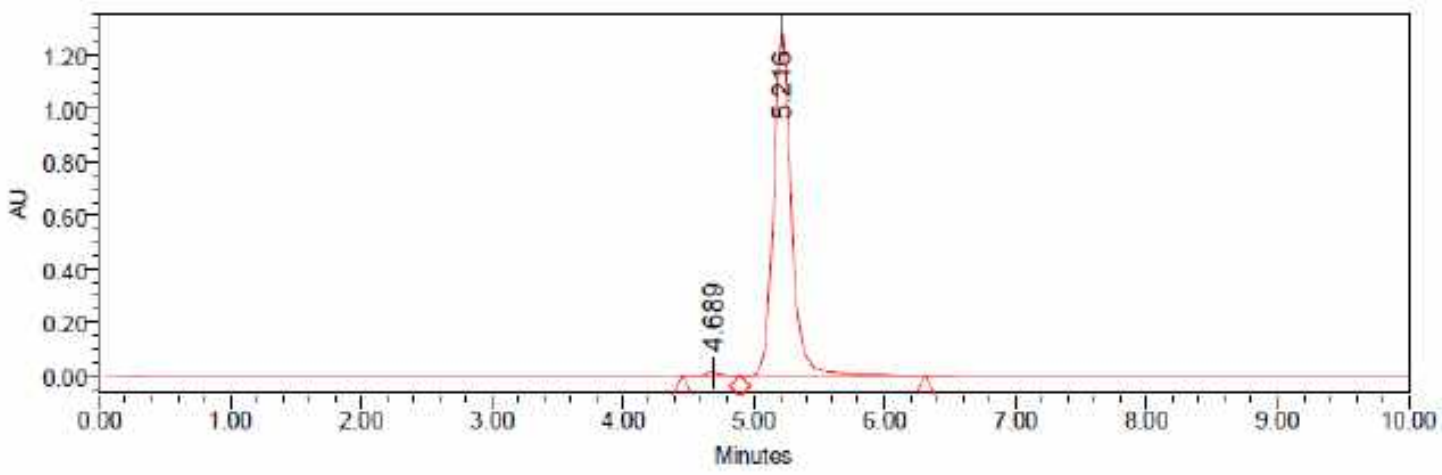

Processed Channel: PDA $225.0 \mathrm{~nm}$

\begin{tabular}{|l|l|r|r|r|r|}
\hline & Processed Channel & $\begin{array}{r}\text { Retention } \\
\text { Time (min) }\end{array}$ & Area & \% Area & Height \\
\hline 1 & PDA 225.0 nm & 4.689 & 110306 & 0.89 & 13260 \\
\hline 2 & PDA 225.0 nm & 5.216 & 12296619 & 99.11 & 1294549 \\
\hline
\end{tabular}


${ }^{1} \mathrm{H}$ NMR $\left(400 \mathrm{MHz}, \mathrm{CDCl}_{3}\right)$ of $\left(S_{a}, R\right)-\mathbf{1 0 b}$ :

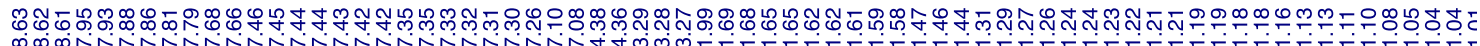

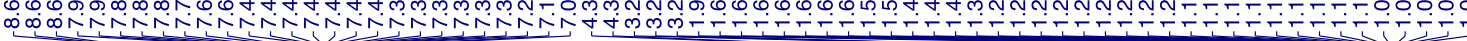

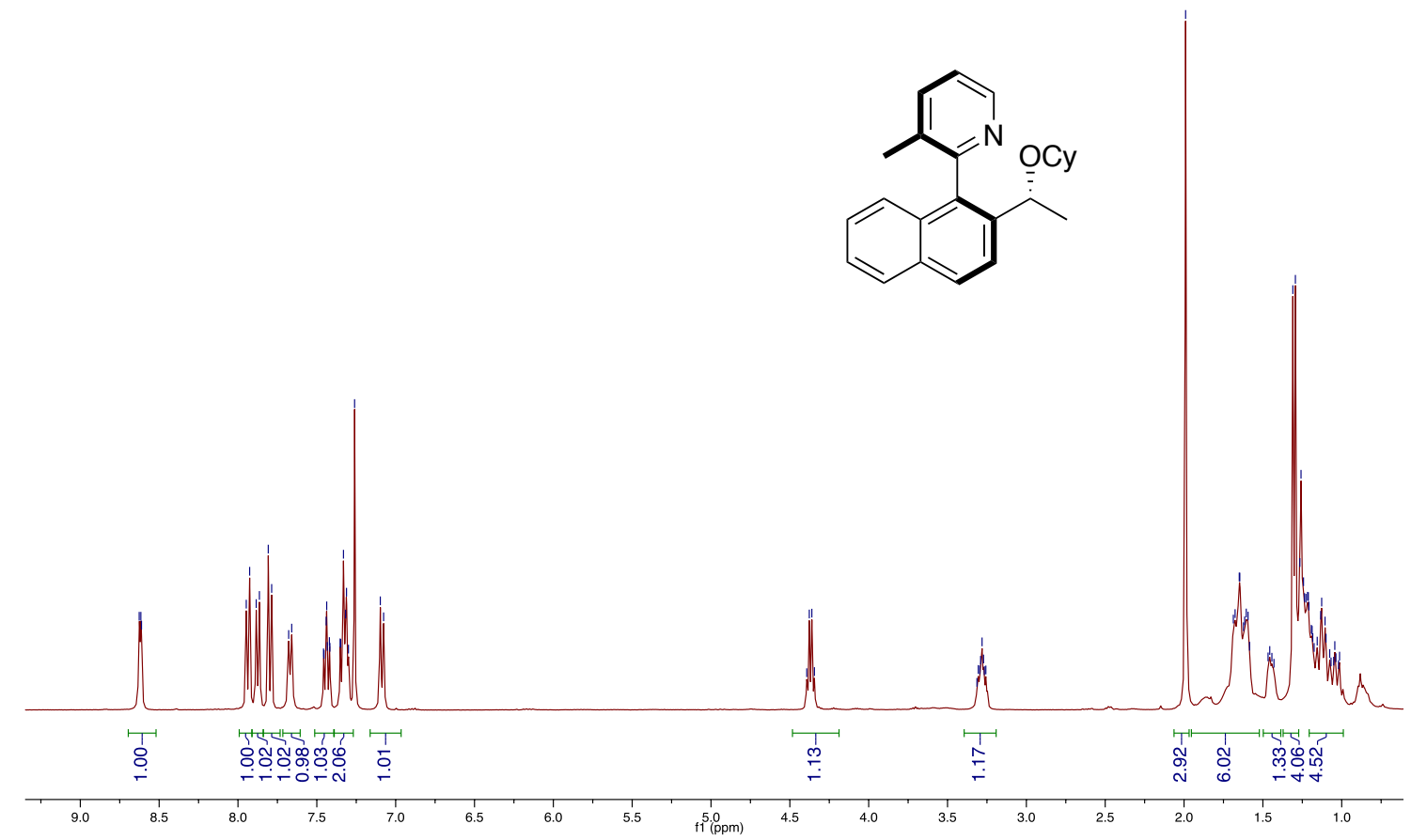

${ }^{13} \mathrm{C}$ NMR $\left(100 \mathrm{MHz}, \mathrm{CDCl}_{3}\right)$ of $\left(S_{a}, R\right)-\mathbf{1 0 b}$ :

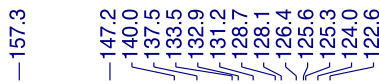

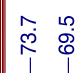

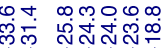

mo niv

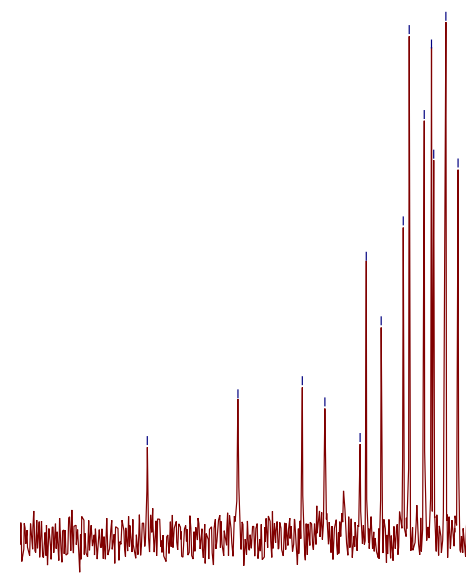<smiles>Cc1cccnc1-c1c(C(C)C)ccc2ccccc12</smiles>

770

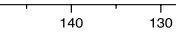

120 
Racemic sample of $( \pm)$-10b: AS-H column, $n$-hexane:isopropanol 99:1, F= $0.5 \mathrm{~mL} / \mathrm{min}$, $\mathrm{T}=30^{\circ} \mathrm{C}$.

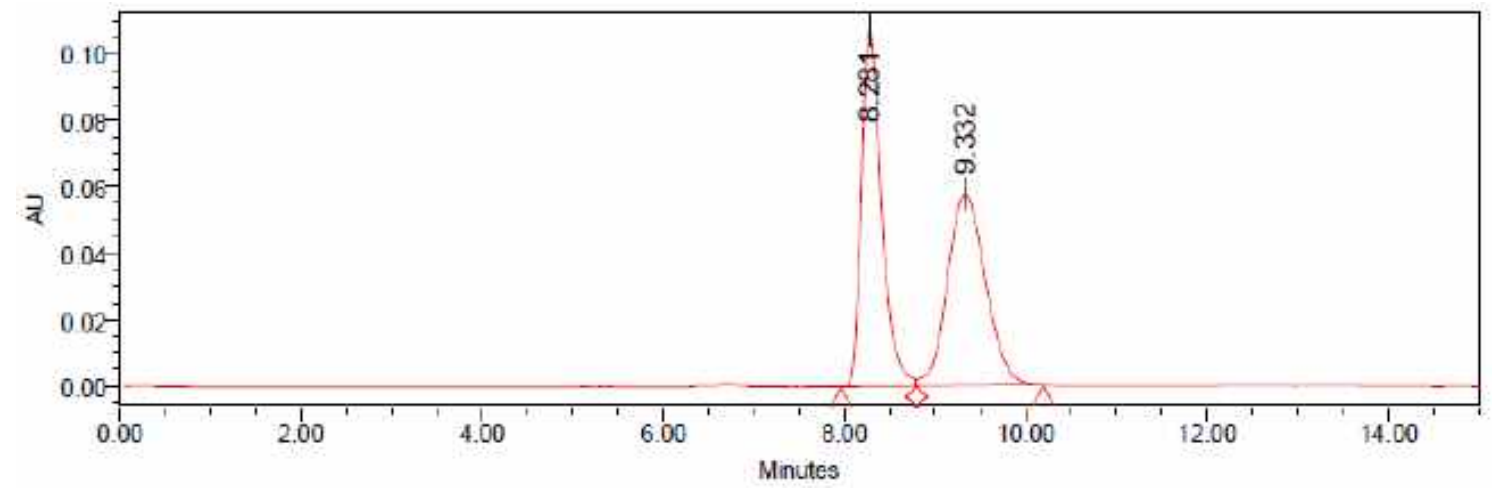

\section{Processed Channel: PDA $224.0 \mathrm{~nm}$}

\begin{tabular}{|l|l|r|r|r|r|}
\hline & Processed Channel & $\begin{array}{r}\text { Retention } \\
\text { Time }(\mathrm{min})\end{array}$ & Area & $\%$ Area & Height \\
\hline 1 & PDA $224.0 \mathrm{~nm}$ & 8.281 & 1637136 & 49.24 & 107363 \\
\hline 2 & PDA $224.0 \mathrm{~nm}$ & 9.332 & 1687778 & 50.76 & 57557 \\
\hline
\end{tabular}

Enantioriched sample of $\left(S_{a}, R\right)-10 \mathrm{~b}$ :

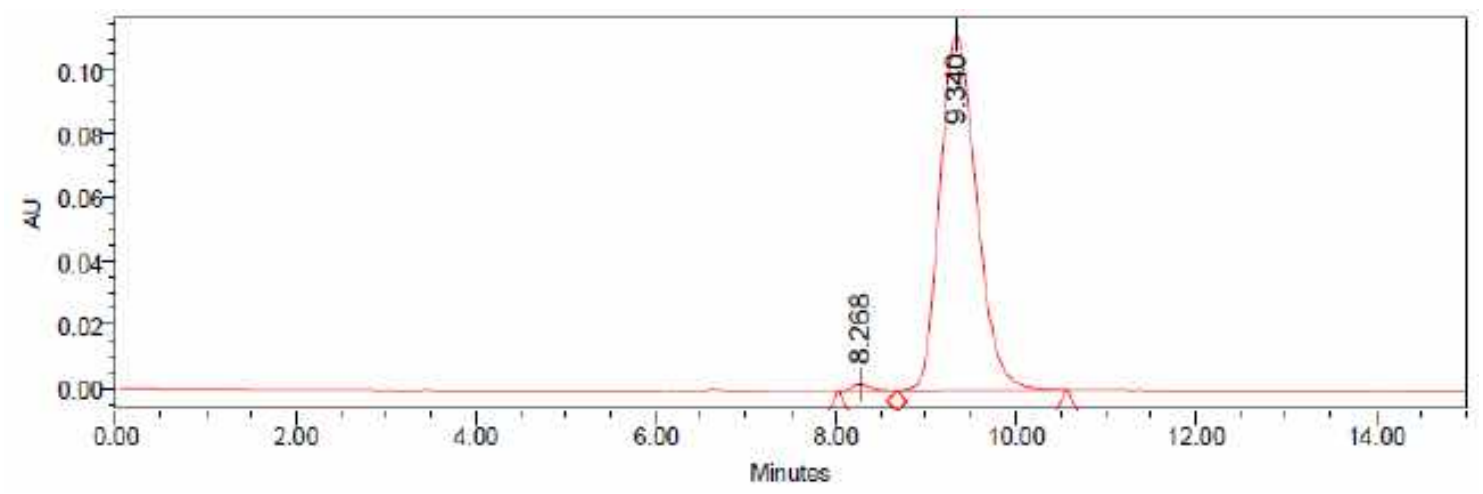

\section{Processed Channel: PDA $224.0 \mathrm{~nm}$}

\begin{tabular}{|l|l|r|r|r|r|}
\hline & Processed Channel & $\begin{array}{r}\text { Retention } \\
\text { Time }(\min )\end{array}$ & Area & \% Area & Height \\
\hline 1 & PDA 224.0 nm & 8.268 & 28297 & 0.89 & 1845 \\
\hline 2 & PDA $224.0 \mathrm{~nm}$ & 9.340 & 3154533 & 99.11 & 111829 \\
\hline
\end{tabular}


${ }^{1} \mathrm{H}$ NMR $\left(400 \mathrm{MHz}, \mathrm{CDCl}_{3}\right)$ of $\left(S_{a}, R\right)-\mathbf{1 0 c}$ :
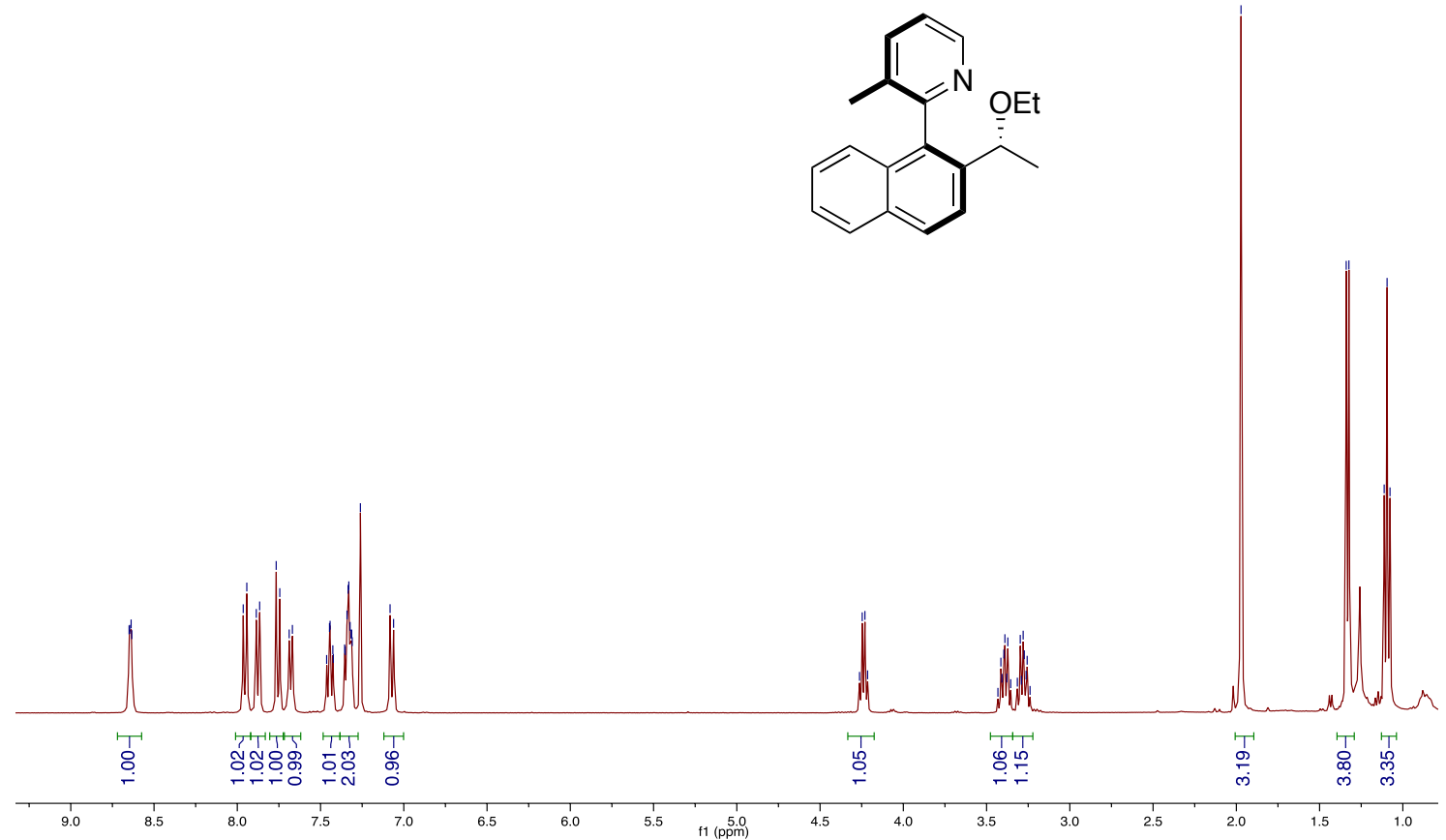

${ }^{13} \mathrm{C}$ NMR $\left(100 \mathrm{MHz}, \mathrm{CDCl}_{3}\right)$ of $\left(S_{a}, R\right)-\mathbf{1 0 c}$ :

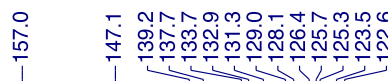

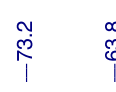

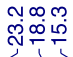
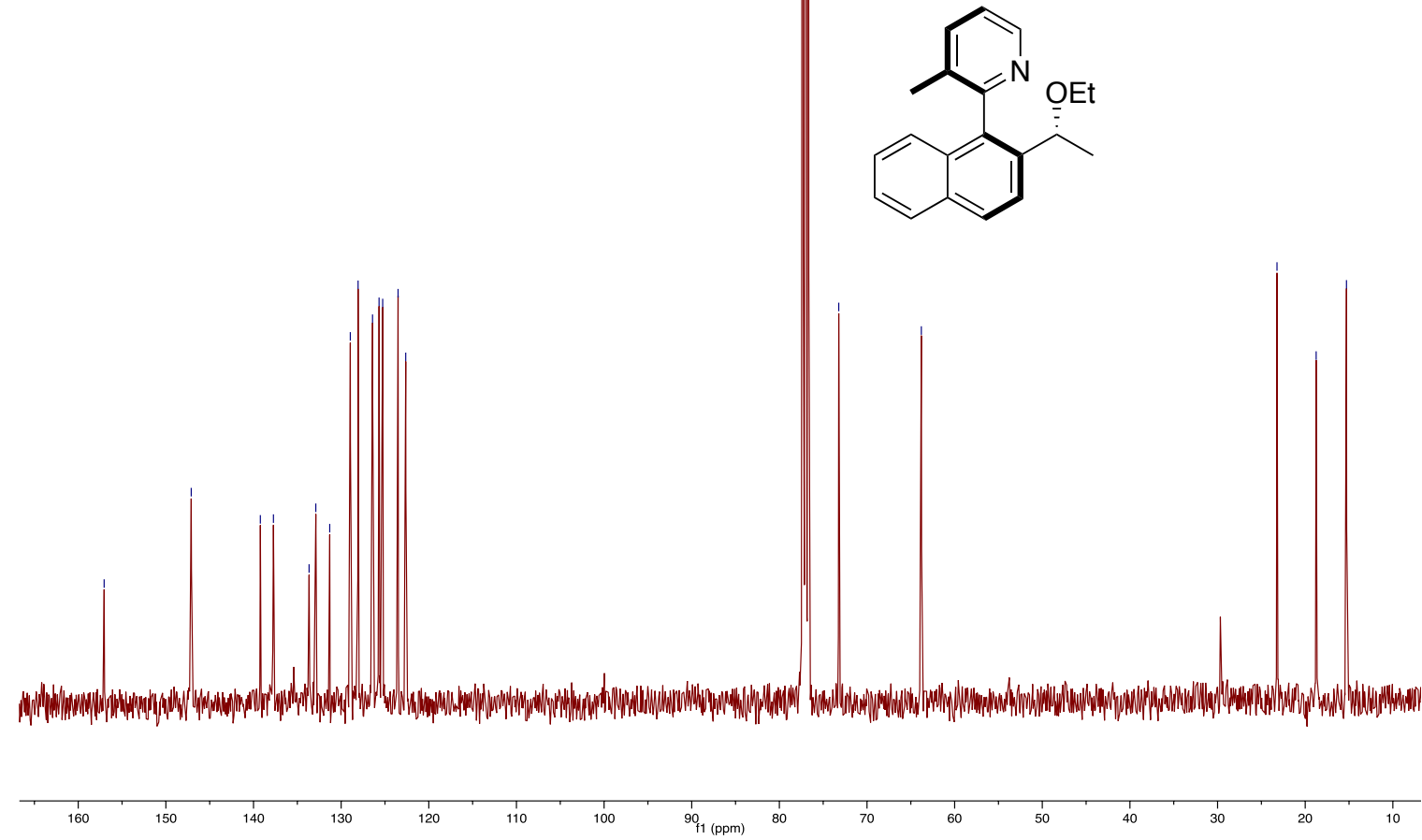
Racemic sample of $( \pm)$-10c: IA column, $n$-hexane:isopropanol 97:3, F= $1 \mathrm{~mL} / \mathrm{min}, \mathrm{T}=$ $30^{\circ} \mathrm{C}$.

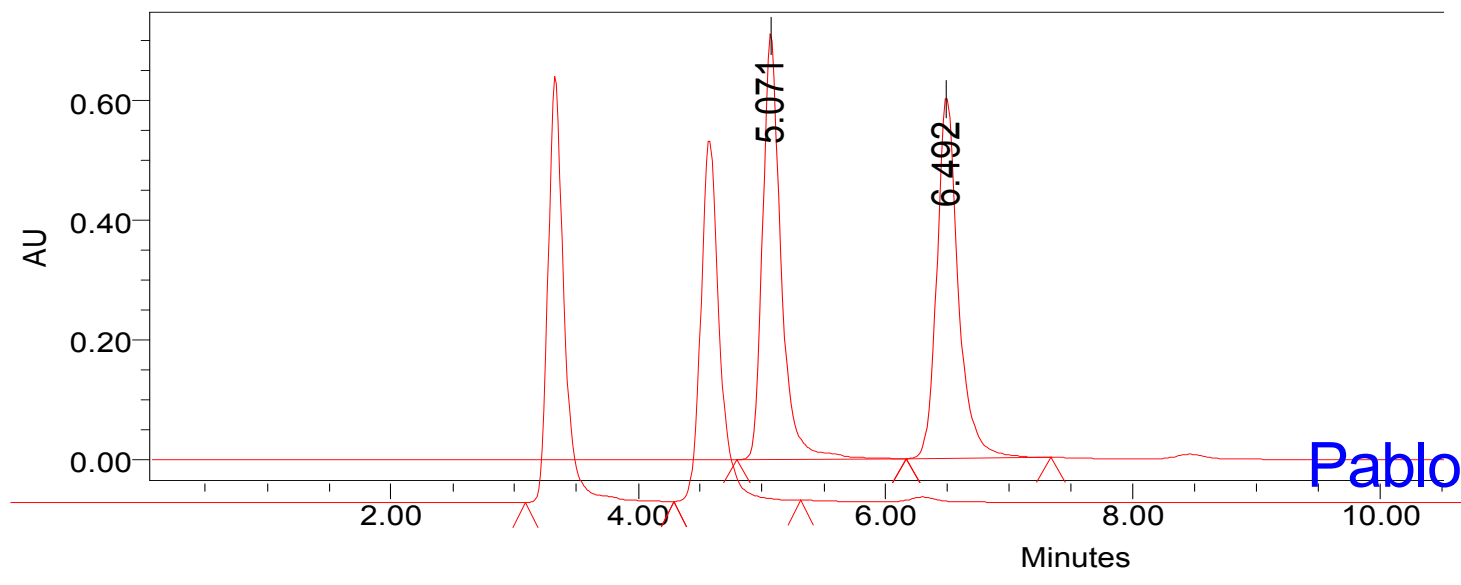

Processed Channel: PDA $224.0 \mathrm{~nm}$

\begin{tabular}{|r|l|r|r|r|r|}
\hline & Processed Channel & $\begin{array}{c}\text { Retention } \\
\text { Time }(\min )\end{array}$ & Area & \% Area & Height \\
\hline 1 & PDA 224.0 nm & 5.071 & 7392982 & 50.39 & 712139 \\
\hline 2 & PDA $224.0 \mathrm{~nm}$ & 6.492 & 7278313 & 49.61 & 603393 \\
\hline
\end{tabular}

Enantioriched sample of $\left(S_{a}, R\right)-10 \mathrm{c}$ :

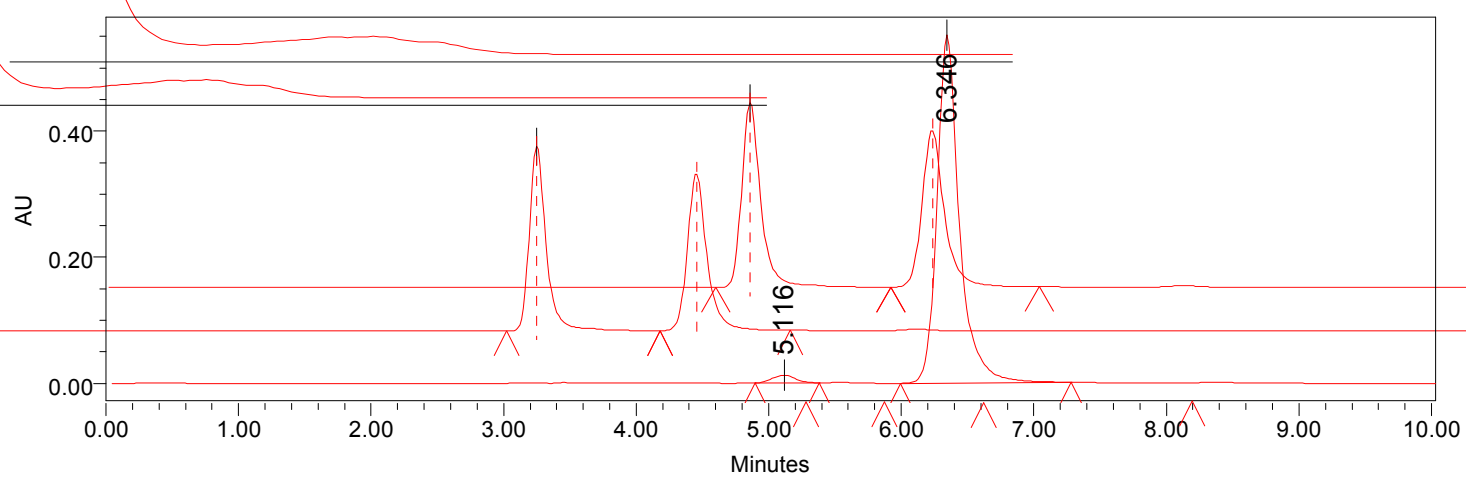

\section{Processed Channel: PDA 222.2 nm}

\begin{tabular}{|r|l|r|r|r|r|}
\hline & Processed Channel & $\begin{array}{r}\text { Retention } \\
\text { Time }(\mathrm{min})\end{array}$ & Area & \% Area & Height \\
\hline 1 & PDA 222.2 nm & 5.116 & 144826 & 2.19 & 11931 \\
\hline 2 & PDA 222.2 nm & 6.346 & 6481744 & 97.81 & 551557 \\
\hline
\end{tabular}


${ }^{1} \mathrm{H}$ NMR $\left(400 \mathrm{MHz}, \mathrm{CDCl}_{3}\right)$ of $\left(S_{a}, R\right)-\mathbf{1 0 d}$ :

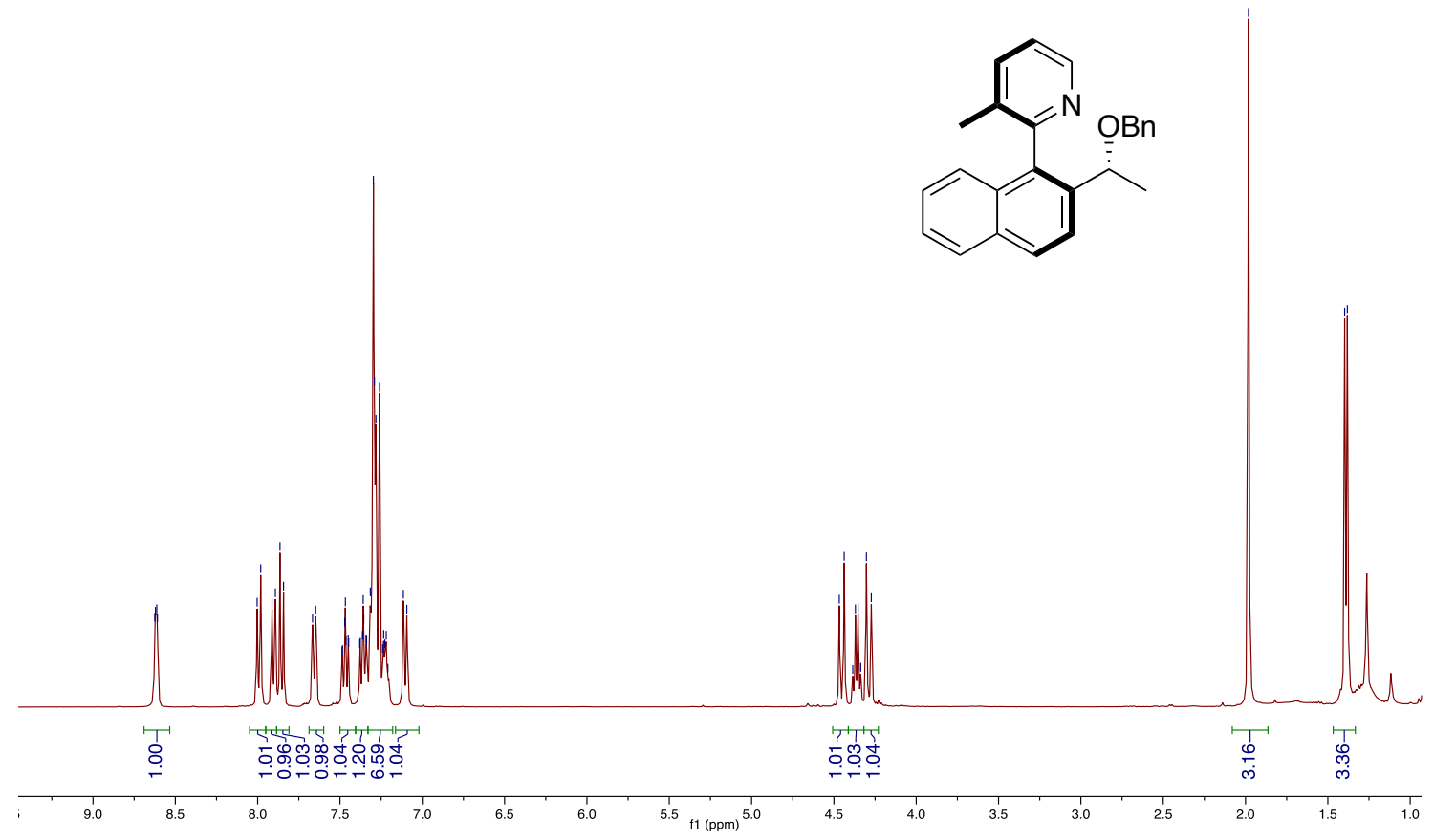

${ }^{13} \mathrm{C}$ NMR $\left(100 \mathrm{MHz}, \mathrm{CDCl}_{3}\right)$ of $\left(S_{a} R\right)$-10d:

蕰

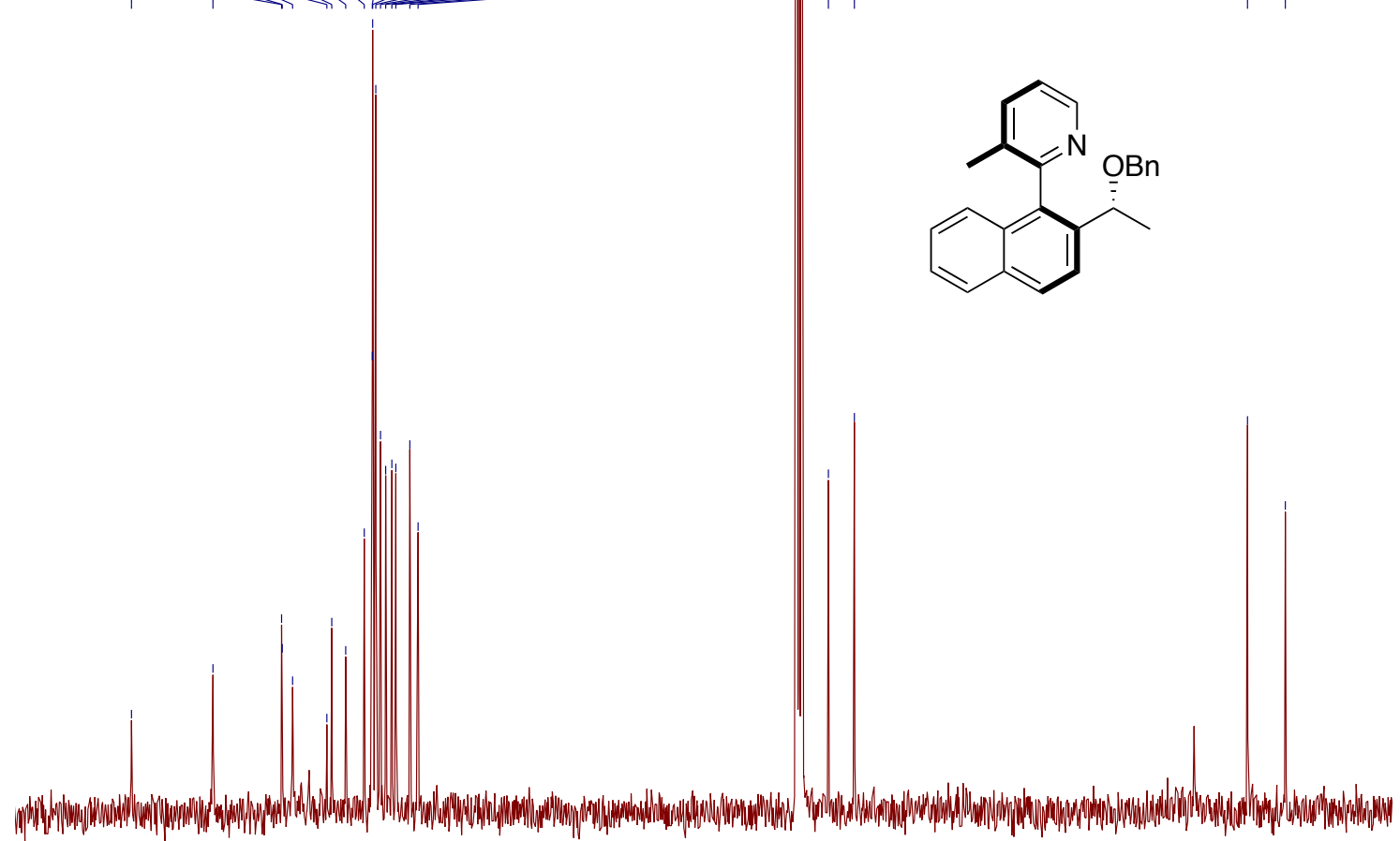

170

150

140

|ึ่

$\stackrel{m}{\stackrel{m}{*}} \hat{\infty}$ 
Racemic sample of $( \pm$ )-10d: OJ-H column, $n$-hexane:isopropanol 95:5, F= $1 \mathrm{~mL} / \mathrm{min}$, $\mathrm{T}=30^{\circ} \mathrm{C}$.

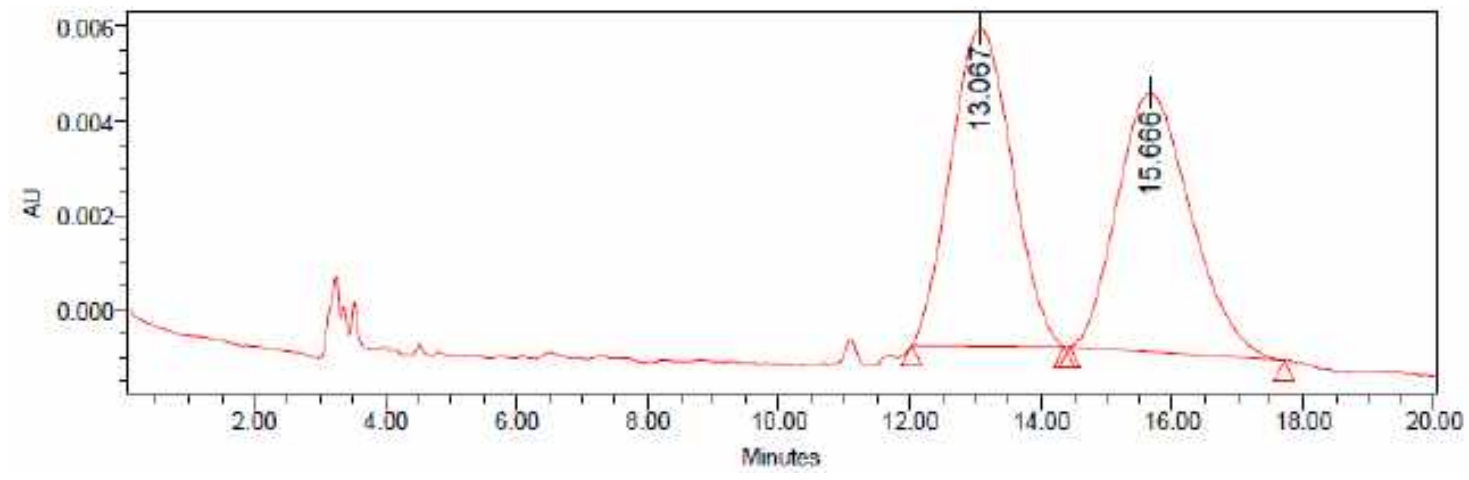

Processed Channel: PDA 225.0 nm
\begin{tabular}{|l|l|r|r|r|r|}
\hline & Processed Channel & $\begin{array}{l}\text { Retention } \\
\text { Time (min) }\end{array}$ & Area & $\%$ Area & Height \\
\hline 1 & PDA $225.0 \mathrm{~nm}$ & 13.067 & 435641 & 50.48 & 6714 \\
\hline 2 & PDA $225.0 \mathrm{~nm}$ & 15.666 & 427336 & 49.52 & 5457 \\
\hline
\end{tabular}

\section{Enantioriched sample of $\left(S_{a}, R\right)$ - 10d:}

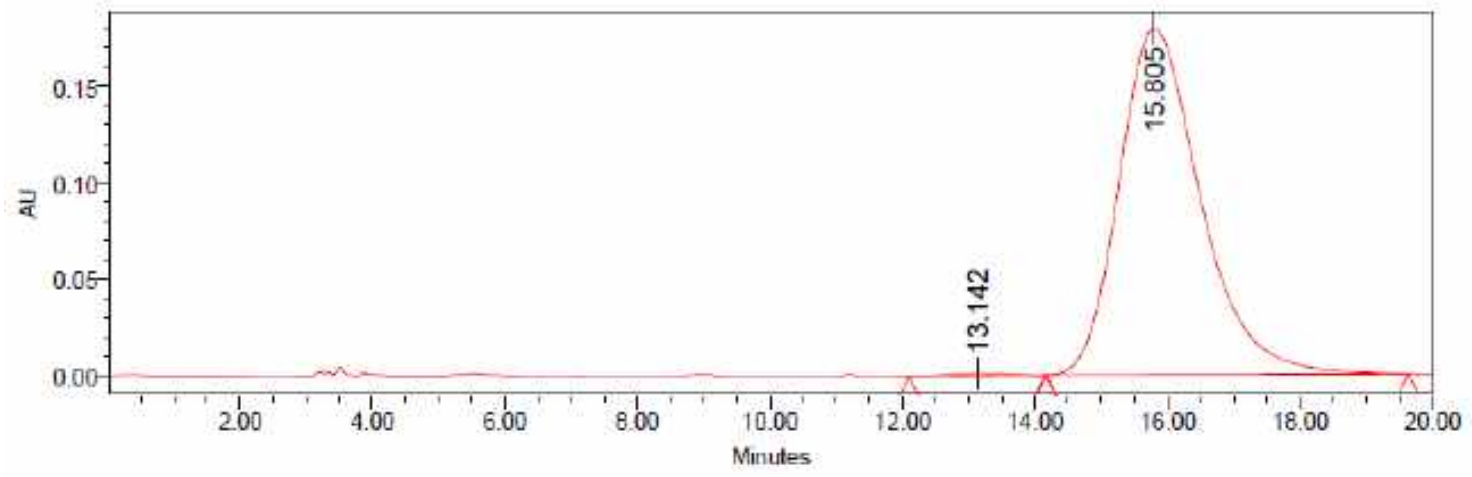

Processed Channel: PDA $225.0 \mathrm{~nm}$

\begin{tabular}{|r|l|r|r|r|r|}
\hline & Processed Channel & $\begin{array}{r}\text { Retention } \\
\text { Time }(\mathrm{min})\end{array}$ & Area & \% Area & Height \\
\hline 1 & PDA 225.0 nm & 13.142 & 71412 & 0.46 & 1223 \\
\hline 2 & PDA 225.0 nm & 15.805 & 15368312 & 99.54 & 179674 \\
\hline
\end{tabular}


${ }^{1} \mathrm{H}$ NMR $\left(400 \mathrm{MHz}, \mathrm{CDCl}_{3}\right)$ of $\left(S_{a}, R\right)-\mathbf{1 0 e}:$

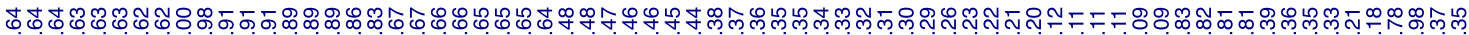

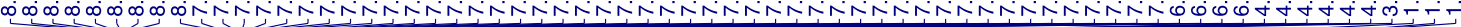

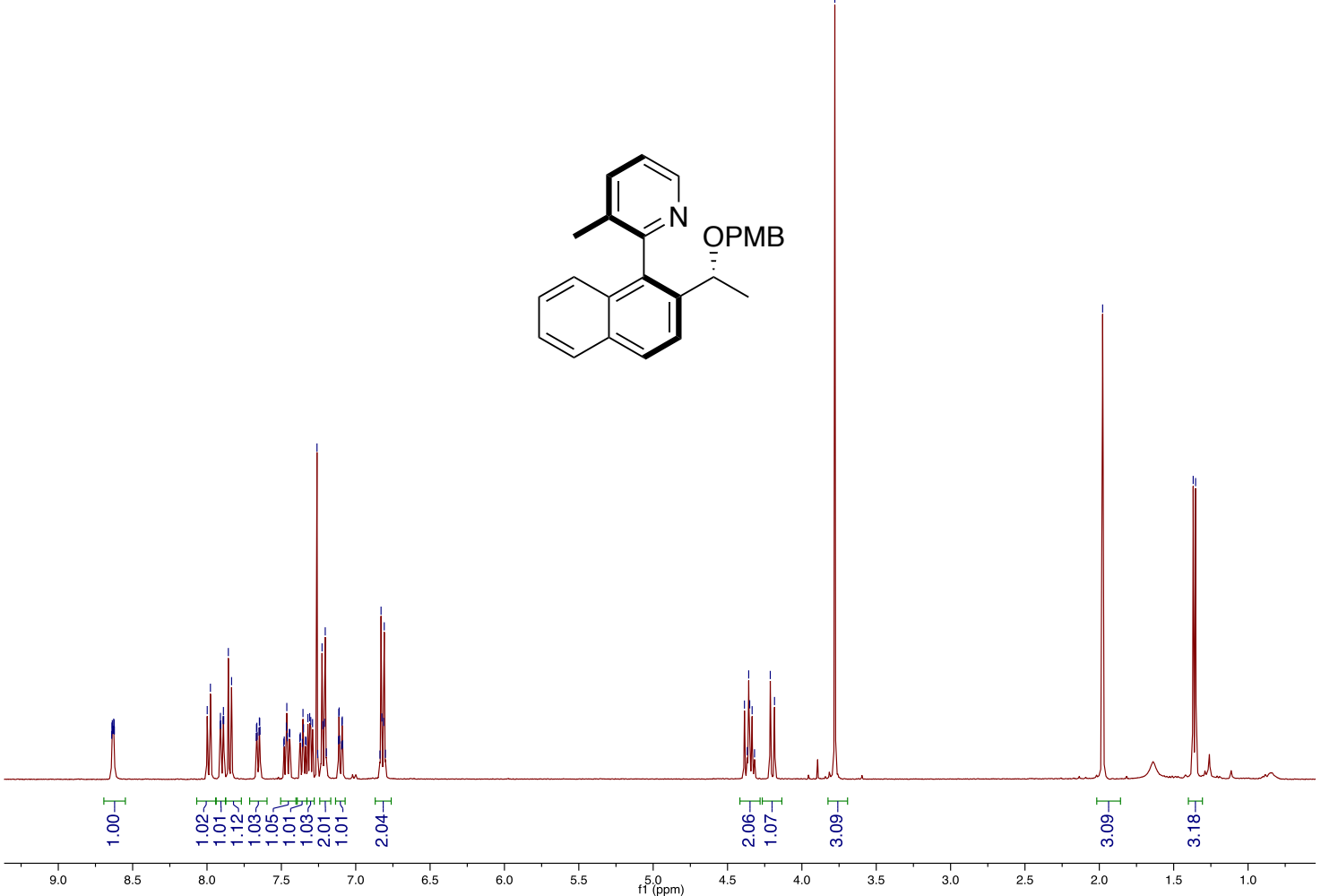

${ }^{13} \mathrm{C}$ NMR $\left(100 \mathrm{MHz}, \mathrm{CDCl}_{3}\right)$ of $\left(S_{a}, R\right)-\mathbf{1 0 e}:$

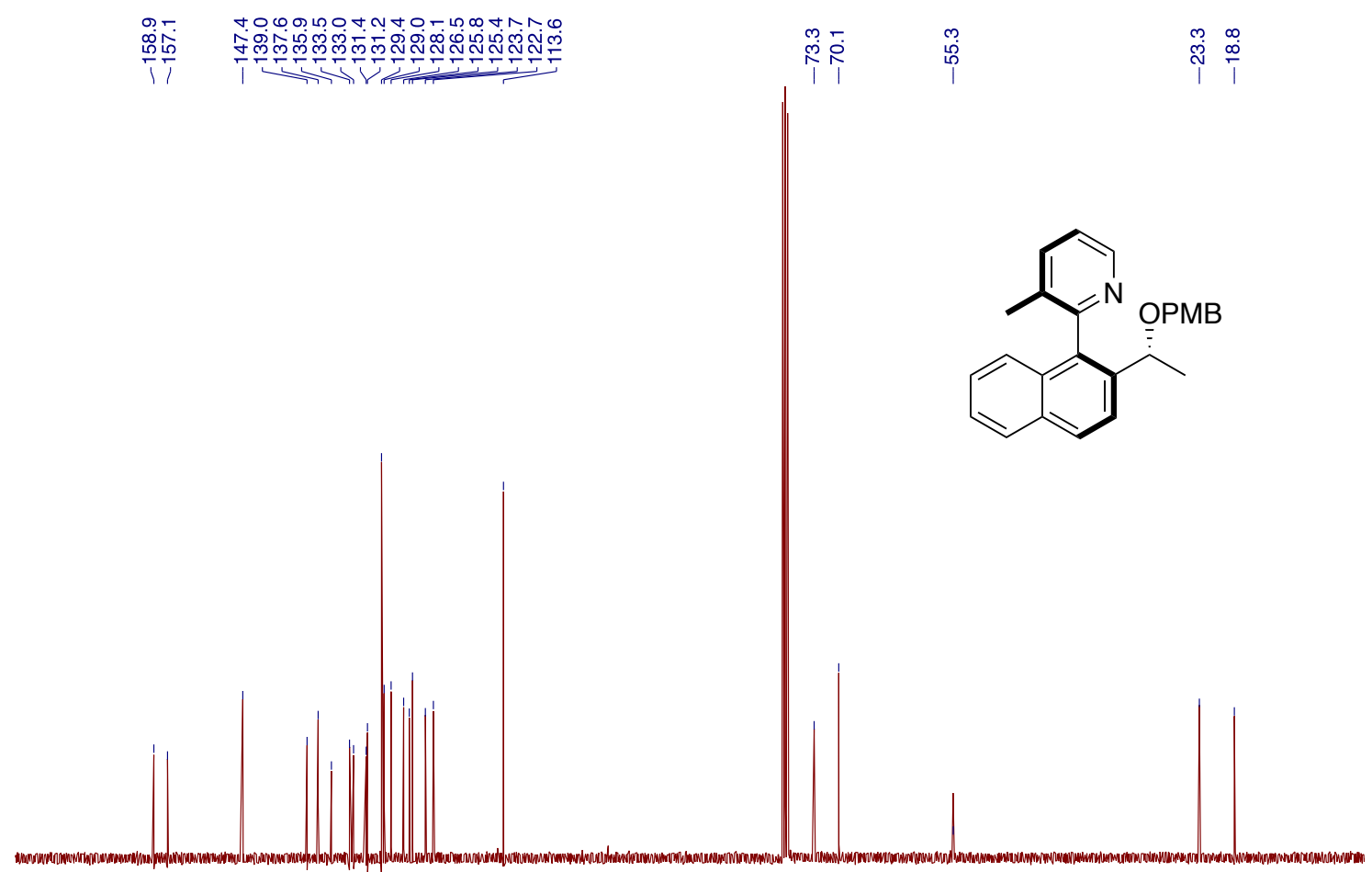


Racemic sample of $( \pm)$-10e: OD column, $n$-hexane:isopropanol 90:10, $\mathrm{F}=1 \mathrm{~mL} / \mathrm{min}$, $\mathrm{T}=30^{\circ} \mathrm{C}$.

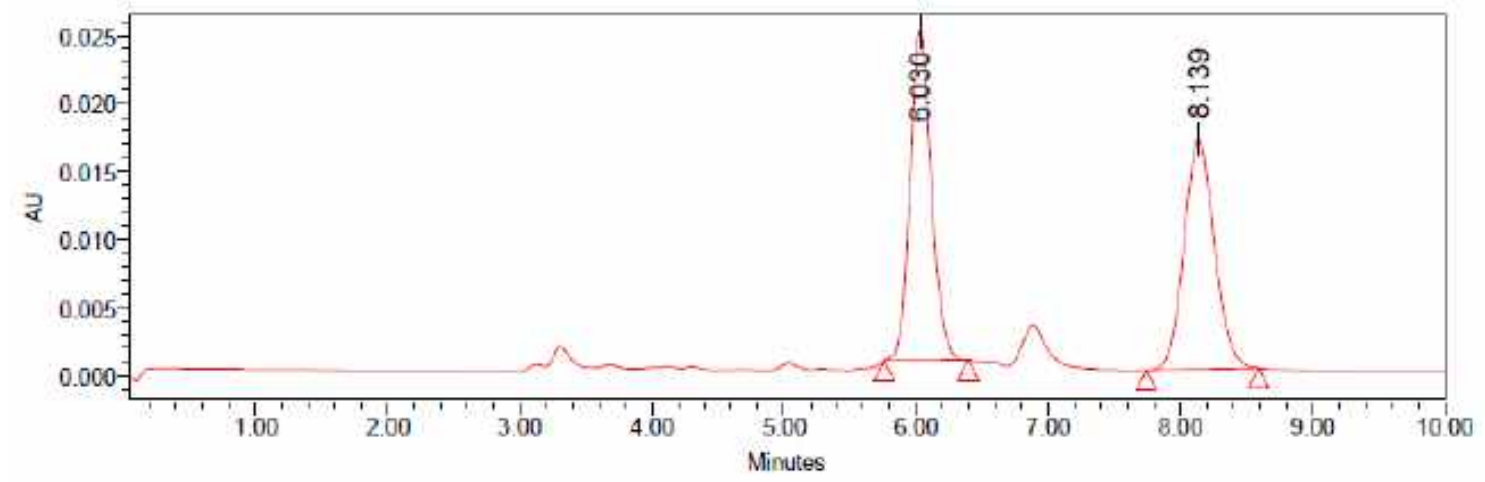

Processed Channel: PDA $236.0 \mathrm{~nm}$

\begin{tabular}{|l|l|r|r|r|r|}
\hline & Processed Channel & $\begin{array}{r}\text { Retention } \\
\text { Time (min) }\end{array}$ & Area & \% Area & Height \\
\hline 1 & PDA $236.0 \mathrm{~nm}$ & 6.030 & 270712 & 50.04 & 24207 \\
\hline 2 & PDA $236.0 \mathrm{~nm}$ & 8.139 & 270310 & 49.96 & 16969 \\
\hline
\end{tabular}

Enantioriched sample of $\left(S_{a}, R\right)-10 \mathrm{e}:$

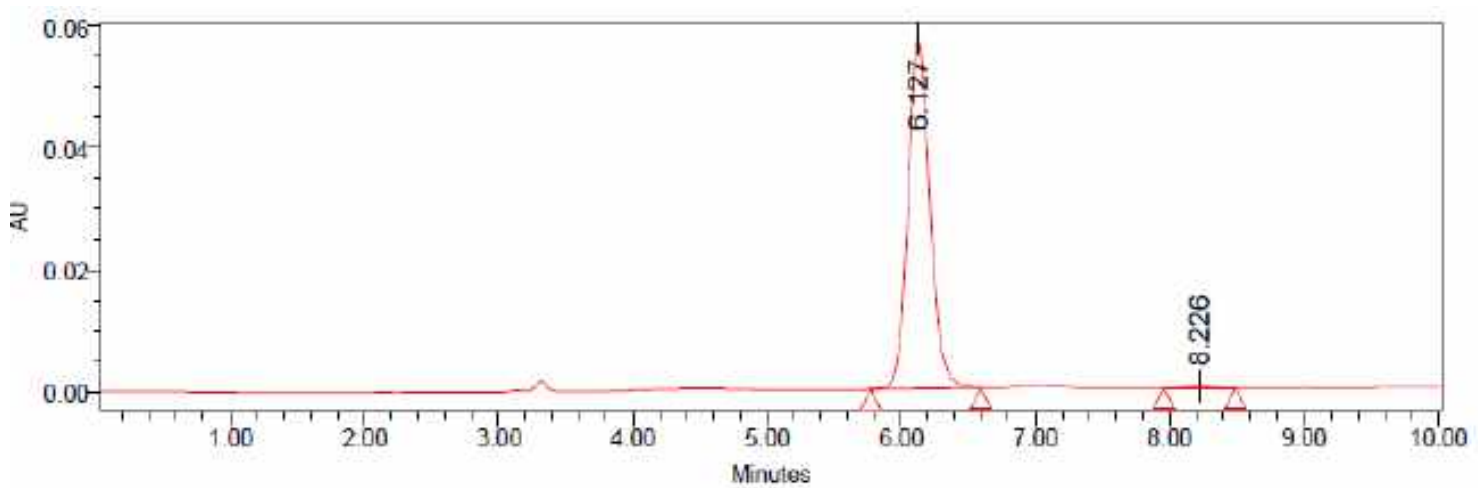

Processed Channel: PDA $236.0 \mathrm{~nm}$

\begin{tabular}{|r|l|r|r|r|r|}
\hline & Processed Channel & $\begin{array}{r}\text { Retention } \\
\text { Time }(\mathrm{min})\end{array}$ & Area & \% Area & Height \\
\hline 1 & PDA $236.0 \mathrm{~nm}$ & 6.127 & 650263 & 99.46 & 57410 \\
\hline 2 & PDA $236.0 \mathrm{~nm}$ & 8.226 & 3555 & 0.54 & 245 \\
\hline
\end{tabular}


${ }^{1} \mathrm{H}$ NMR $\left(400 \mathrm{MHz}, \mathrm{CDCl}_{3}\right)$ of $\left(S_{a}, R\right)-\mathbf{1 0 f}$ :

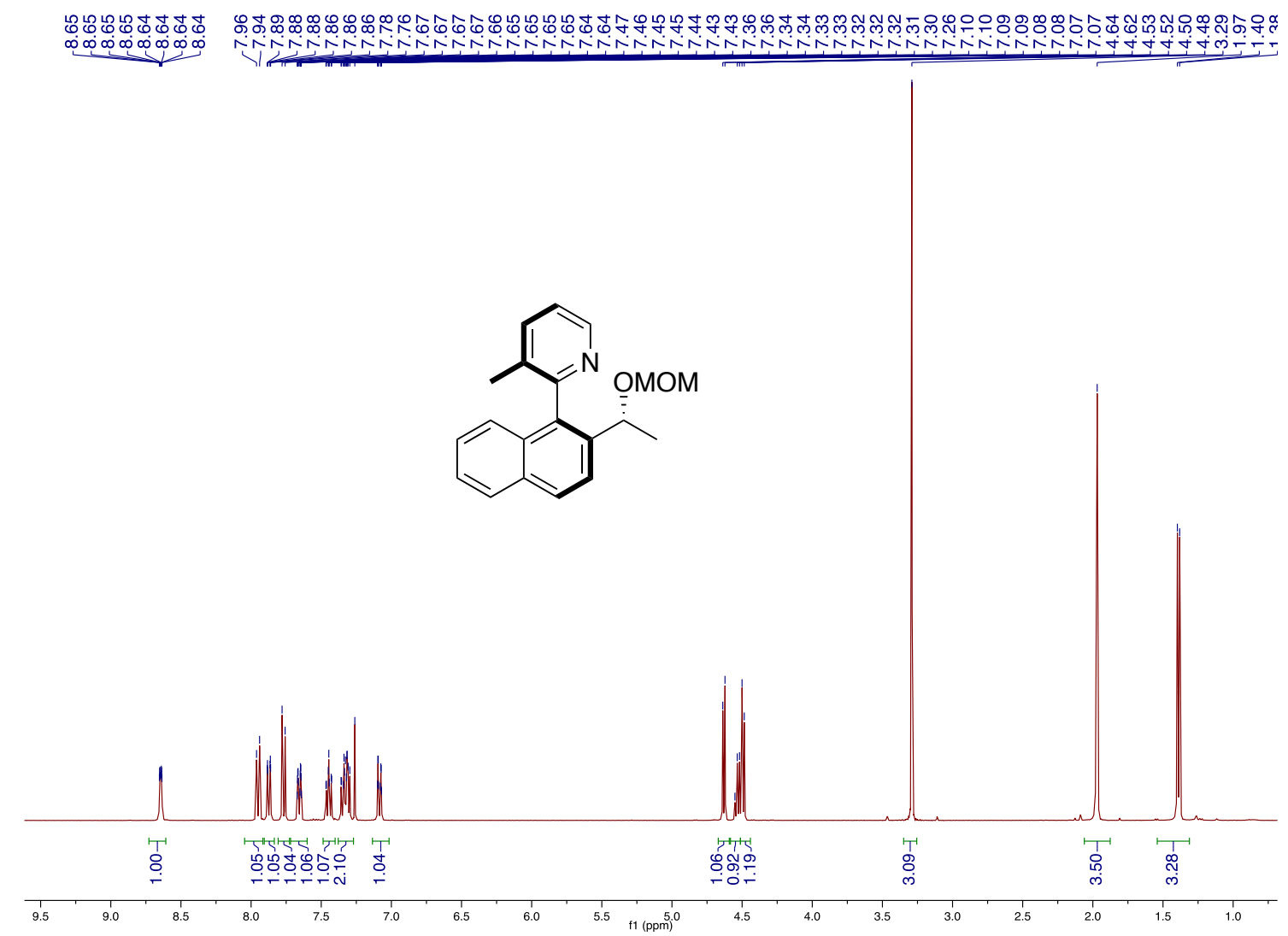

${ }^{13} \mathrm{C}$ NMR $\left(100 \mathrm{MHz}, \mathrm{CDCl}_{3}\right)$ of $\left(S_{a}, R\right)-\mathbf{1 0 f}$ :

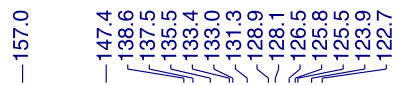

官

余苫

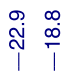
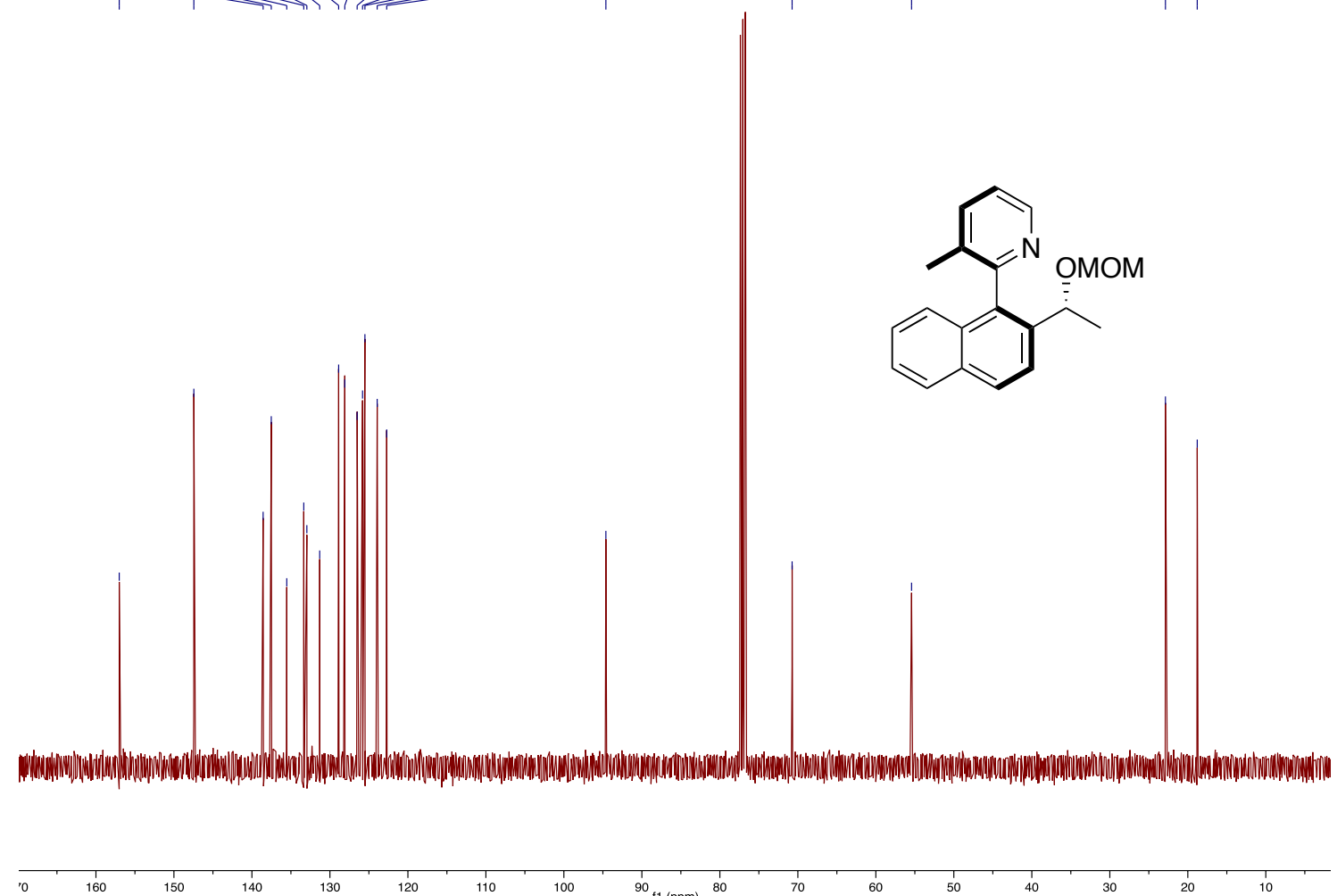

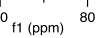


Racemic sample of $( \pm)$-10f: IA column, $n$-hexane:isopropanol 95:5, F= $1 \mathrm{~mL} / \mathrm{min}, \mathrm{T}=$ $30^{\circ} \mathrm{C}$.

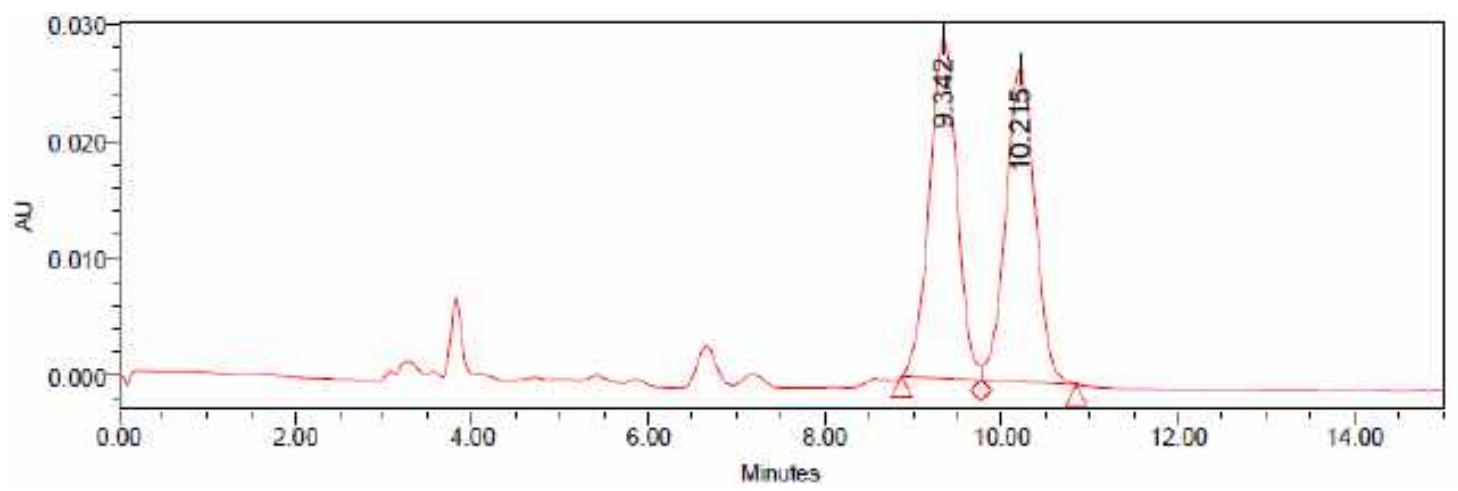

\section{Processed Channel: PDA $231.0 \mathrm{~nm}$}

\begin{tabular}{|l|l|r|c|r|r|}
\hline & Processed Channel & $\begin{array}{c}\text { Retention } \\
\text { Time }(\text { min) }\end{array}$ & Area & \% Area & Height \\
\hline 1 & PDA $231.0 \mathrm{~nm}$ & 9.342 & 634407 & 50.44 & 28936 \\
\hline 2 & PDA $231.0 \mathrm{~nm}$ & 10.215 & 623456 & 49.56 & 26760 \\
\hline
\end{tabular}

Enantioriched sample $\left(S_{a}, R\right)-\mathbf{1 0 f :}$

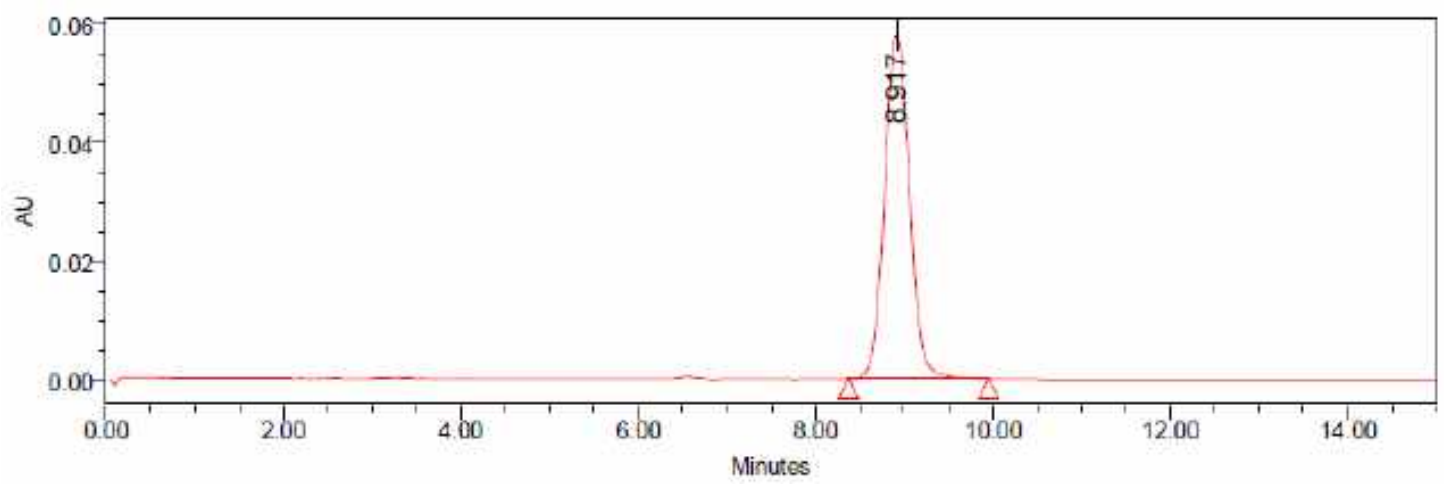

Processed Channel: PDA 231.0 nm
\begin{tabular}{|l|r|r|r|r|r|}
\hline & Processed Channel & $\begin{array}{r}\text { Retention } \\
\text { Time (min) }\end{array}$ & Area & $\%$ Area & Height \\
\hline 1 & PDA $231.0 \mathrm{~nm}$ & 8.917 & 1130507 & 100.00 & 57799 \\
\hline
\end{tabular}


${ }^{1} \mathrm{H} \mathrm{NMR}\left(400 \mathrm{MHz}, \mathrm{CDCl}_{3}\right)$ of the minor diastereomer of $\mathbf{1 0 g}$ :

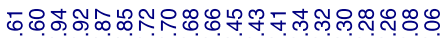

onn

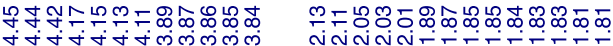<smiles>Cc1cccnc1-c1c(C2CCCO2)ccc2ccccc12</smiles>

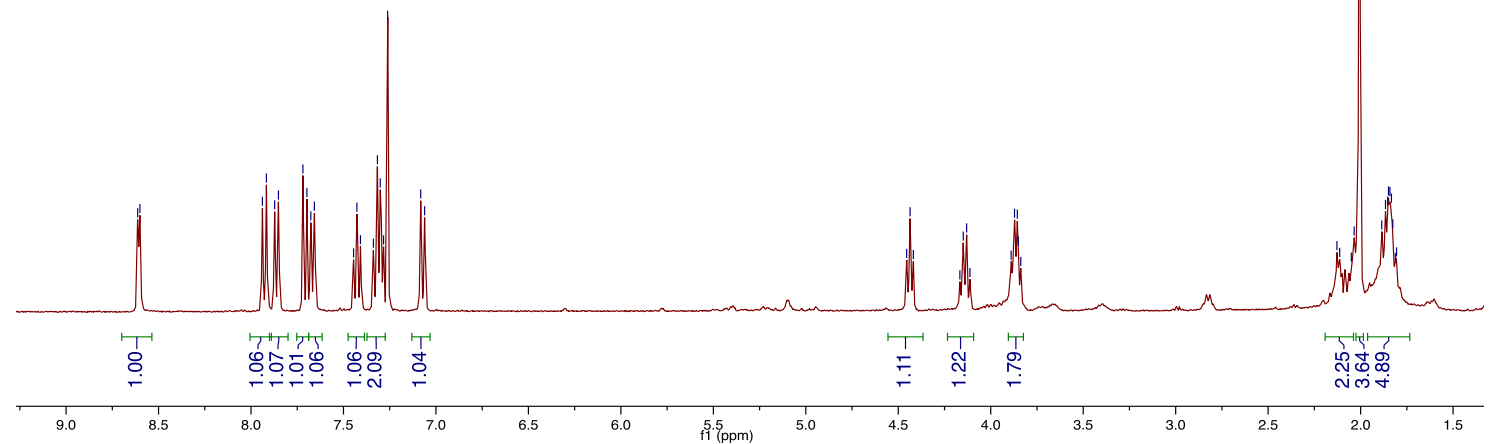

${ }^{13} \mathrm{C} \mathrm{NMR}\left(100 \mathrm{MHz}, \mathrm{CDCl}_{3}\right)$ of the minor diastereomer of $\mathbf{1 0 g}$ :
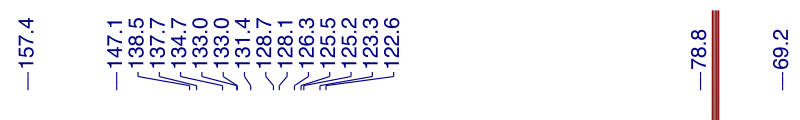

$\underset{\dot{m}}{\bar{m}} \stackrel{0}{\stackrel{\infty}{i}} \stackrel{\infty}{\infty}$<smiles>Cc1cccnc1-c1c(C2CCCO2)ccc2ccccc12</smiles>
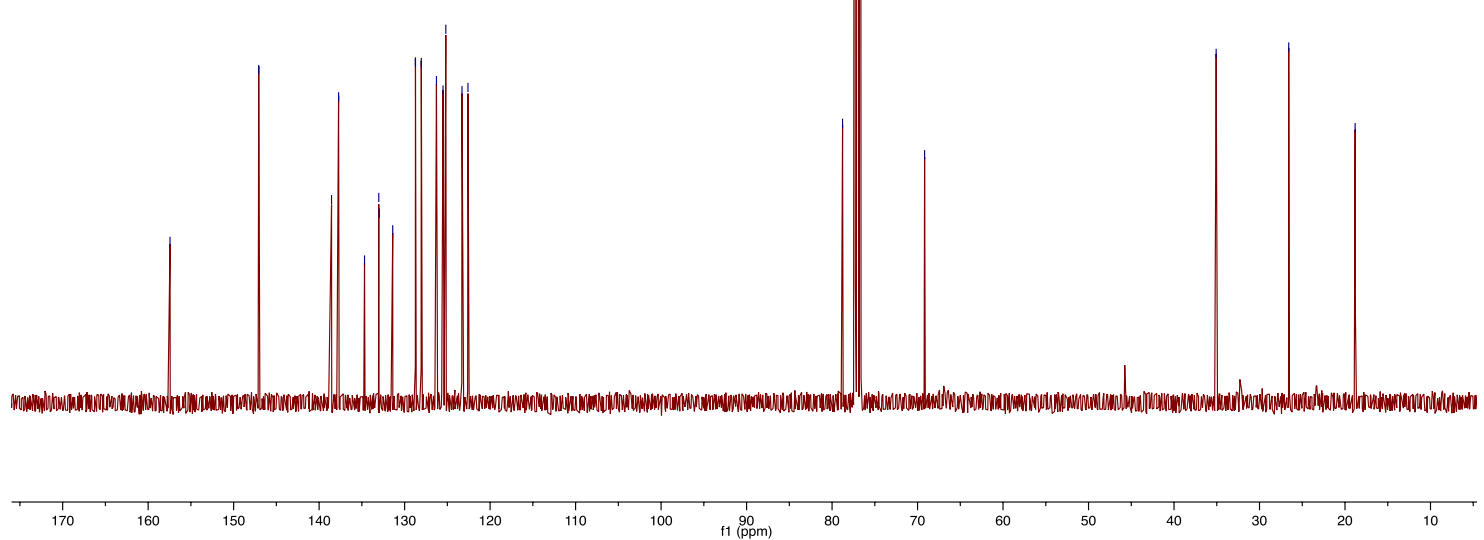
Racemic sample of the minor diastereomer of $( \pm)-10 g$ : IB column, $n$ hexane:isopropanol 99:1, $\mathrm{F}=1 \mathrm{~mL} / \mathrm{min}, \mathrm{T}=30^{\circ} \mathrm{C}$.

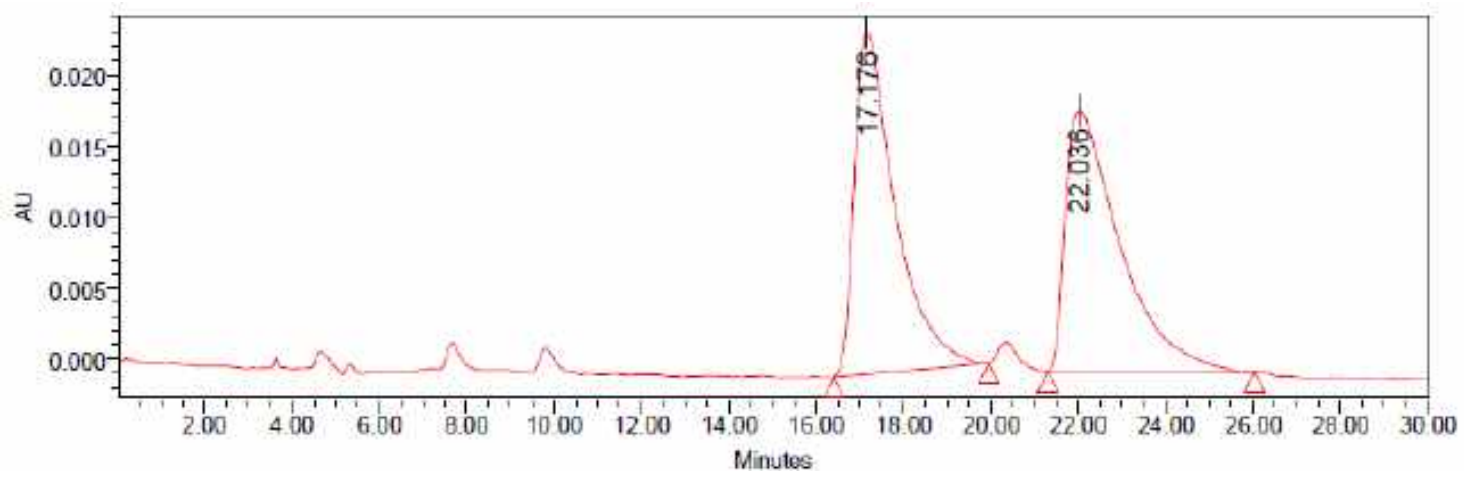

Processed Channel: PDA $225.0 \mathrm{~nm}$

\begin{tabular}{|l|l|r|r|r|r|}
\hline & Processed Channel & $\begin{array}{r}\text { Retention } \\
\text { Time (min) }\end{array}$ & Area & \% Area & Height \\
\hline 1 & PDA 225.0 nm & 17.176 & 1560765 & 49.56 & 24015 \\
\hline 2 & PDA 225.0 nm & 22.036 & 1588399 & 50.44 & 18342 \\
\hline
\end{tabular}

\section{Enantioriched sample of the minor diastereomer of $10 \mathrm{~g}$ :}

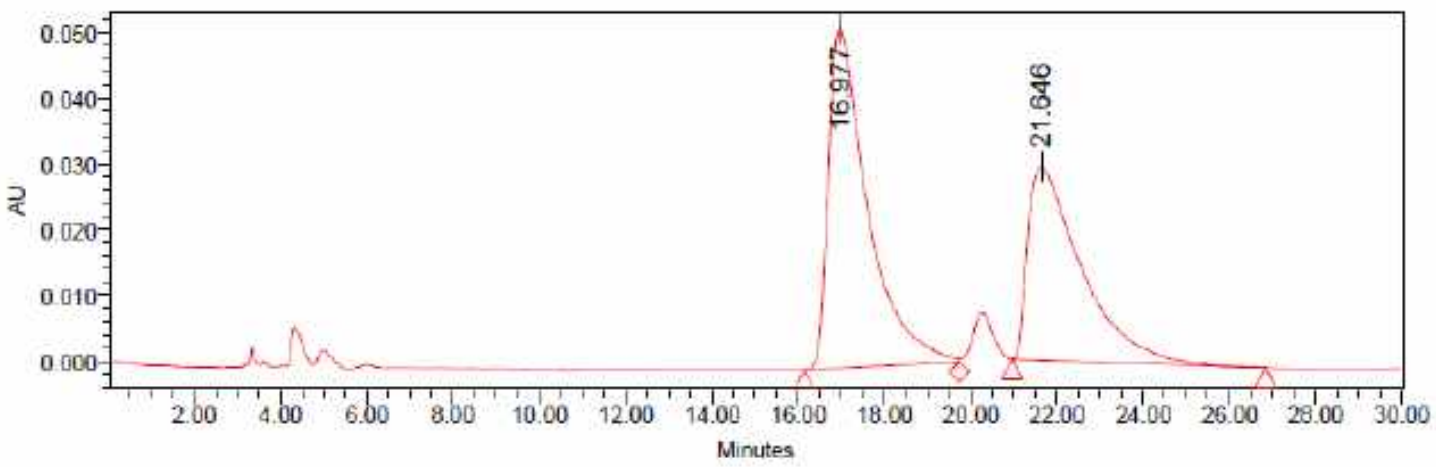

Processed Channel: PDA $225.0 \mathrm{~nm}$

\begin{tabular}{|l|l|r|r|r|r|}
\hline & Processed Channel & $\begin{array}{r}\text { Retention } \\
\text { Time }(\min )\end{array}$ & Area & \% Area & Height \\
\hline 1 & PDA $225.0 \mathrm{~nm}$ & 16.977 & 3261490 & 56.31 & 51620 \\
\hline 2 & PDA $225.0 \mathrm{~nm}$ & 21.646 & 2530855 & 43.69 & 29272 \\
\hline
\end{tabular}


${ }^{1} \mathrm{H}$ NMR $\left(400 \mathrm{MHz}, \mathrm{CDCl}_{3}\right)$ of $\left(R_{a}, S\right) \mathbf{- 1 0 g}$ :<smiles>Cc1cccnc1-c1c(C2CCCO2)ccc2ccccc12</smiles>
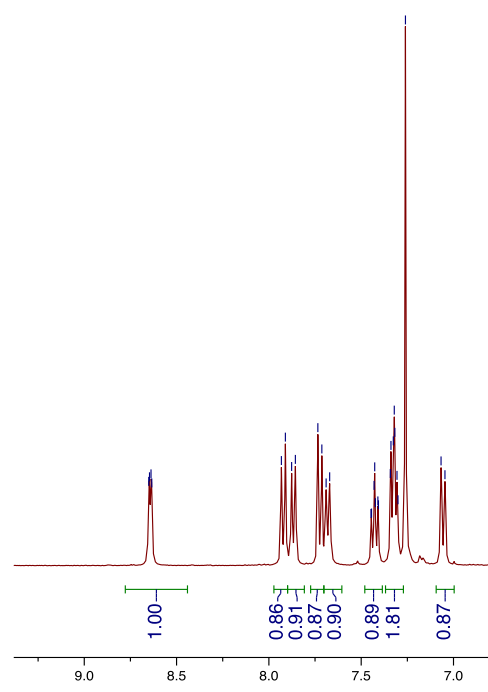

${ }^{13} \mathrm{C}$ NMR $\left(100 \mathrm{MHz}, \mathrm{CDCl}_{3}\right)$ of $\left(R_{a}, S\right)-\mathbf{1 0 g}$ :

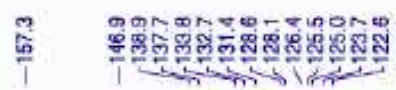<smiles>Cc1cccnc1-c1c(C2CCCO2)ccc2ccccc12</smiles>
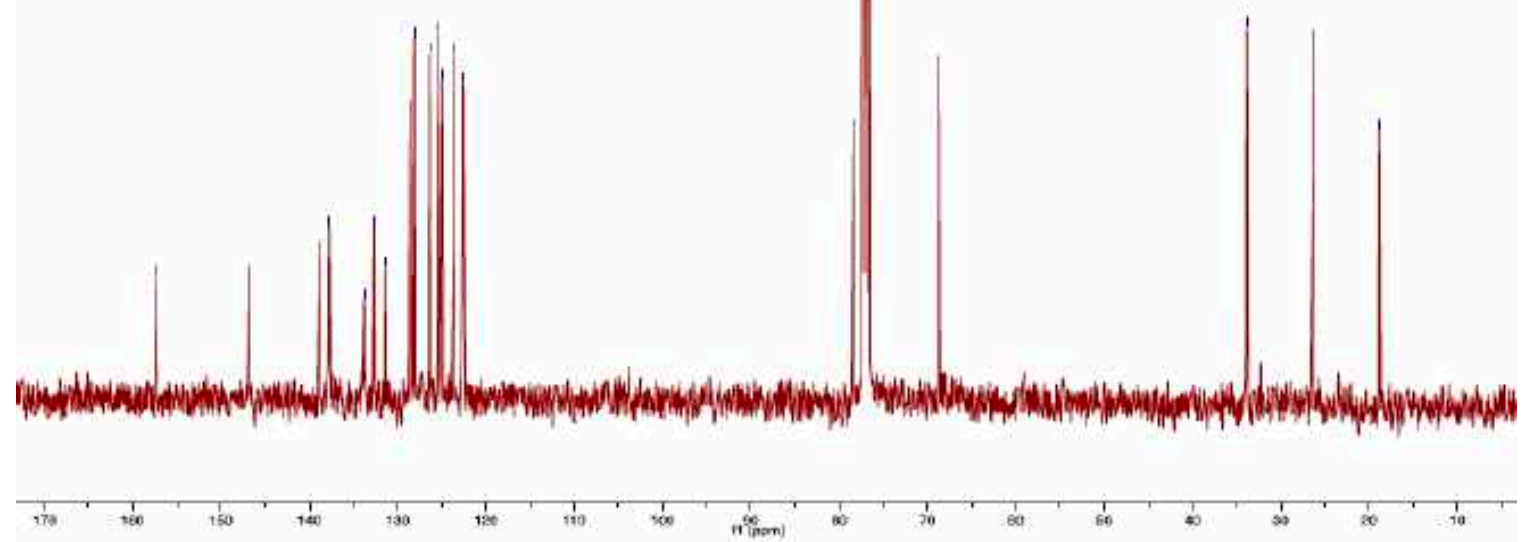
Racemic sample of the major diastereomer of $( \pm)-10 g$ : IA column, $n$ hexane:isopropanol 90:10, $\mathrm{F}=1 \mathrm{~mL} / \mathrm{min}, \mathrm{T}=30^{\circ} \mathrm{C}$.

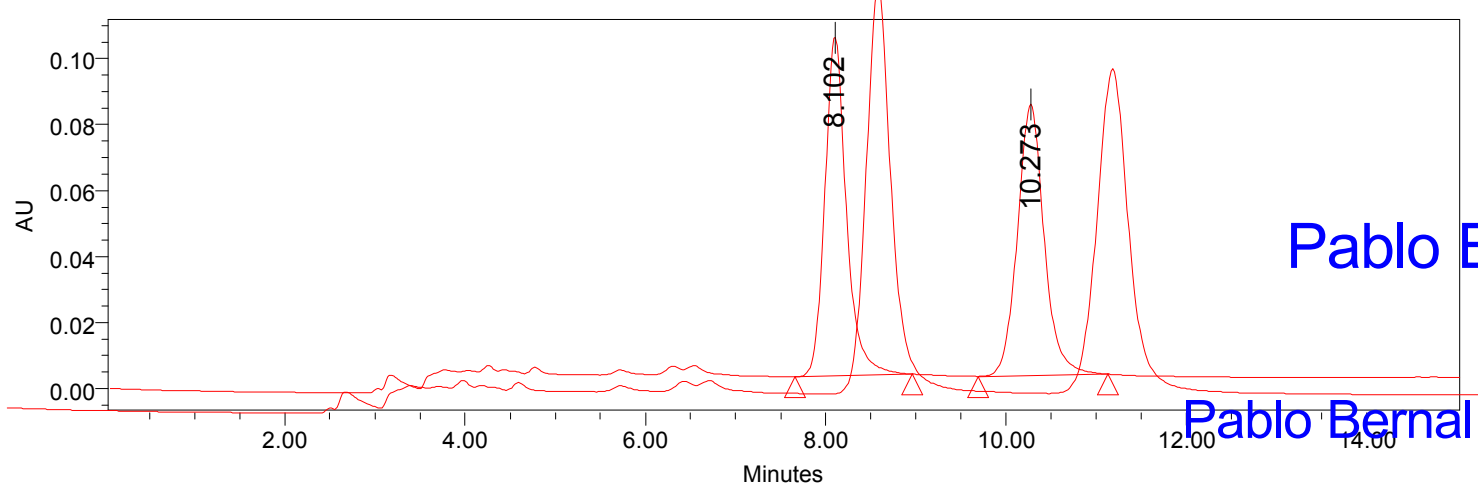

Processed Channel: PDA 226.0 nm

\begin{tabular}{|r|l|r|c|r|r|}
\hline & Processed Channel & $\begin{array}{c}\text { Retention } \\
\text { Time (min) }\end{array}$ & Area & \% Area & Height \\
\hline 1 & PDA 226.0 nm & 8.102 & 1712924 & 50.24 & 102785 \\
\hline 2 & PDA 226.0 nm & 10.273 & 1696555 & 49.76 & 82254 \\
\hline
\end{tabular}

Enantioriched sample of the major diastereomer of $\left(R_{a}, S\right)-10 \mathrm{~g}$ :

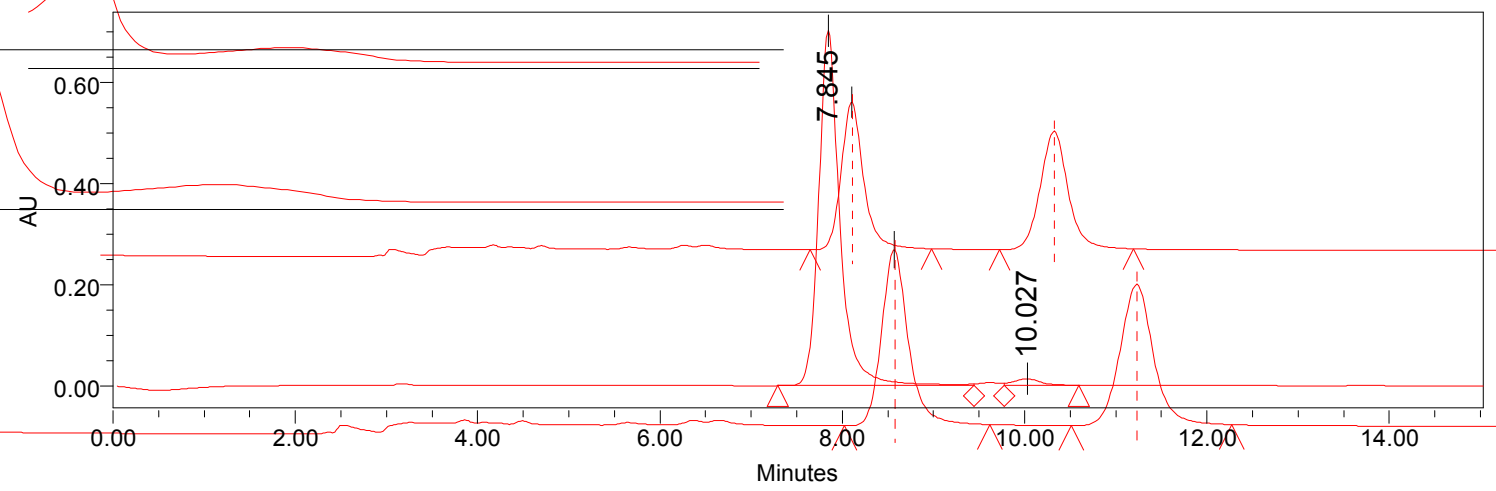

Processed Channel: PDA 226.0 nm

\begin{tabular}{|r|l|r|r|r|r|}
\hline & Processed Channel & $\begin{array}{r}\text { Retention } \\
\text { Time }(\mathrm{min})\end{array}$ & Area & \% Area & Height \\
\hline 1 & PDA 226.0 nm & 7.845 & 11309489 & 97.76 & 701140 \\
\hline 2 & PDA 226.0 nm & 10.027 & 259389 & 2.24 & 12909 \\
\hline
\end{tabular}


${ }^{1} \mathrm{H}$ NMR $\left(400 \mathrm{MHz}, \mathrm{CDCl}_{3}\right)$ of $\left(S_{a}, R\right)-\mathbf{1 1 a}$ :

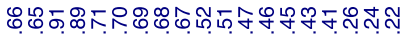

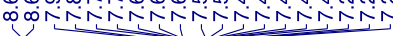

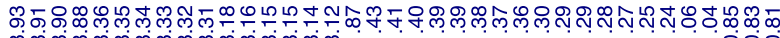

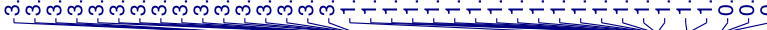
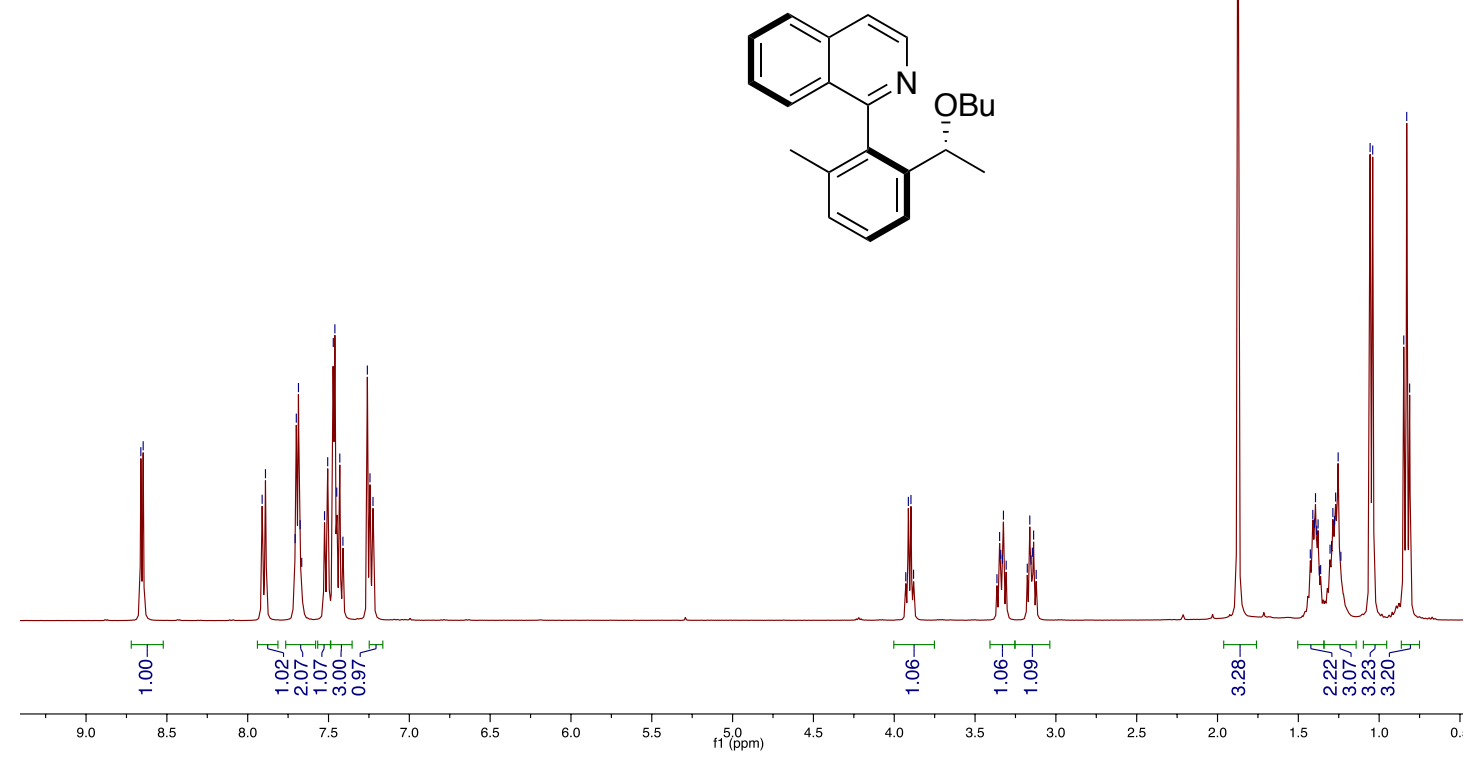

${ }^{13} \mathrm{C}$ NMR $\left(100 \mathrm{MHz}, \mathrm{CDCl}_{3}\right)$ of $\left(S_{a}, R\right)$-11a:

: إن

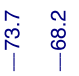

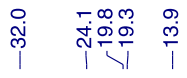<smiles>CCCC(C)c1cccc(C)c1-c1nccc2ccccc12</smiles>

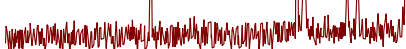
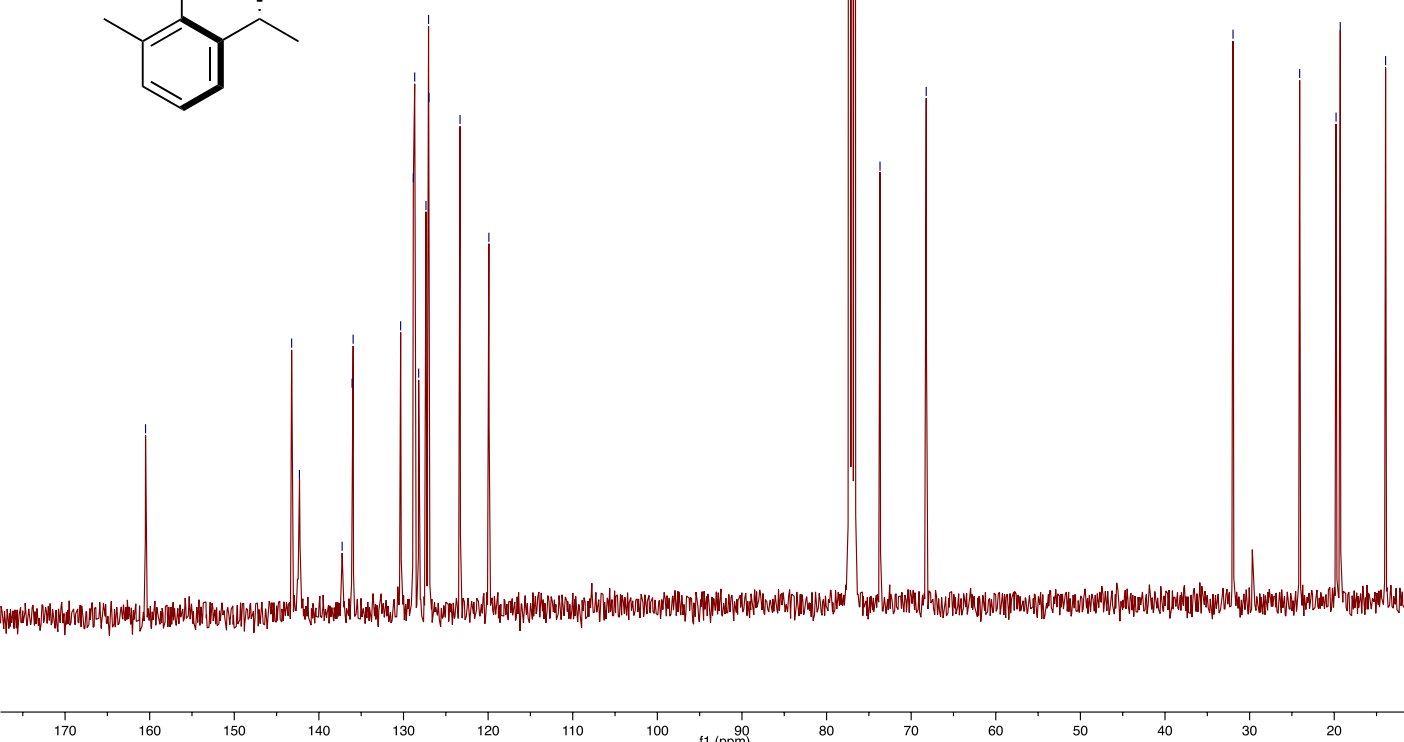

110

$100 \quad \mathrm{f1}(\mathrm{pp}$ 
Racemic sample of $( \pm)$-11a: OD-H column, $n$-hexane:isopropanol 99:1, F= $1 \mathrm{~mL} / \mathrm{min}$, $\mathrm{T}=30^{\circ} \mathrm{C}$.

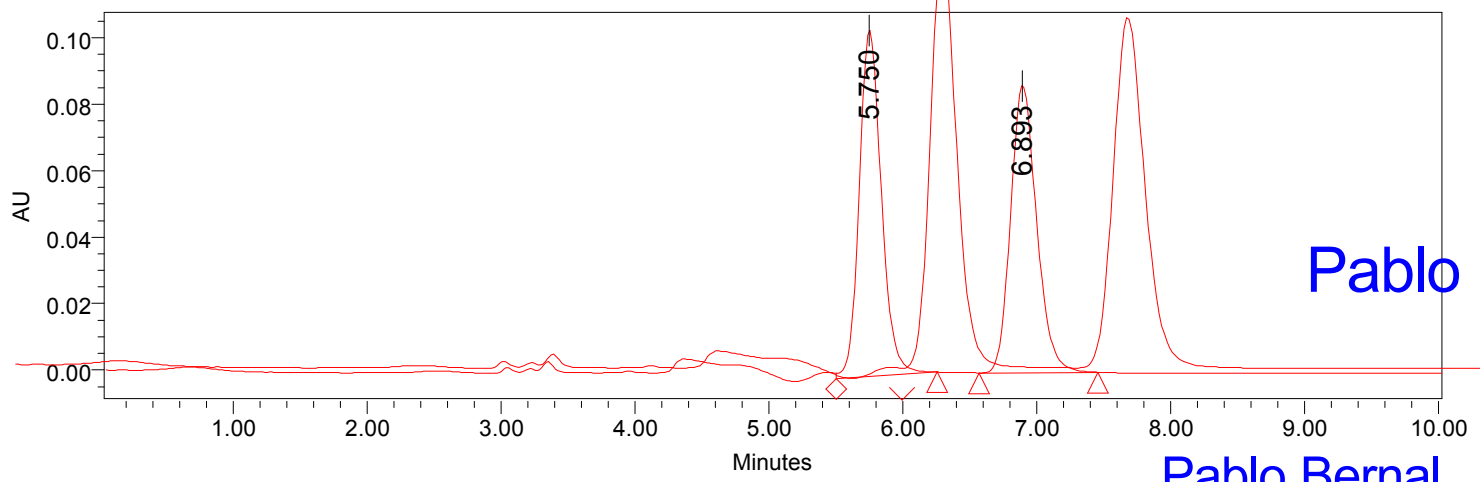

\section{Processed Channel: PDA 215.5 nm}

\begin{tabular}{|r|l|r|r|r|r|}
\hline & Processed Channel & $\begin{array}{r}\text { Retention } \\
\text { Time }(\min )\end{array}$ & Area & \% Area & Height \\
\hline 1 & PDA 215.5 nm & 5.750 & 1196305 & 50.63 & 104332 \\
\hline 2 & PDA 215.5 nm & 6.893 & 1166393 & 49.37 & 86567 \\
\hline
\end{tabular}

Enantioriched sample of $\left(S_{a}, R\right)-11 a$ :

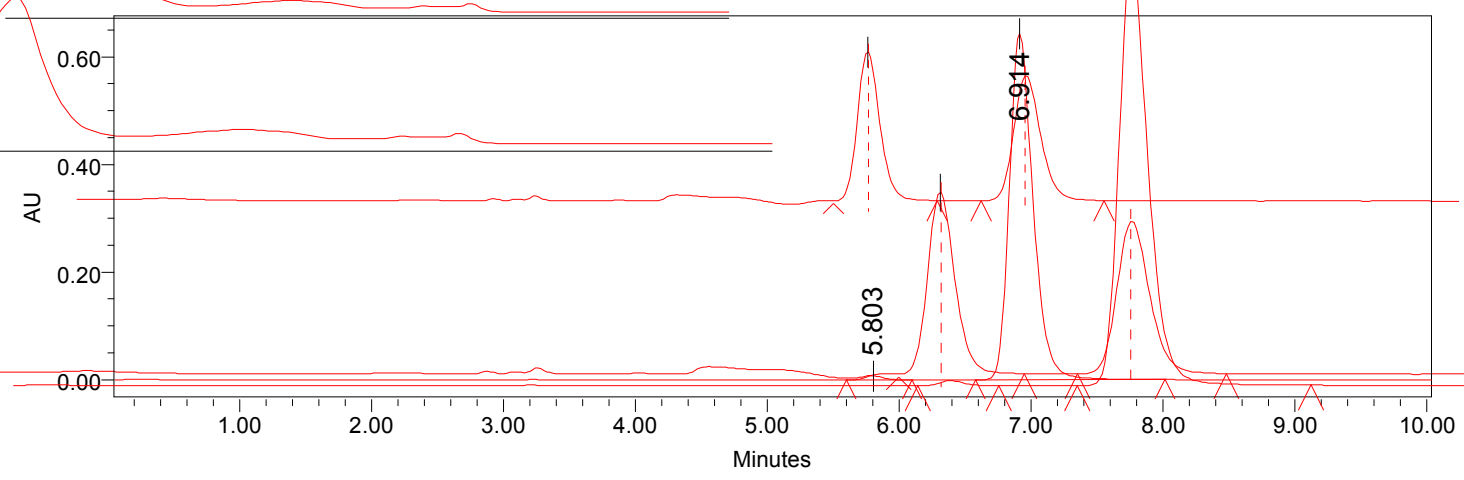

\section{Processed Channel: PDA 218.0 nm}

\begin{tabular}{|r|l|r|r|r|r|}
\hline & Processed Channel & $\begin{array}{r}\text { Retention } \\
\text { Time }(\mathrm{min})\end{array}$ & Area & \% Area & Height \\
\hline 1 & PDA 218.0 nm & 5.803 & 66384 & 0.82 & 7036 \\
\hline 2 & PDA 218.0 nm & 6.914 & 8055295 & 99.18 & 643823 \\
\hline
\end{tabular}


$\left.{ }^{1} \mathrm{H} \mathrm{NMR} \mathrm{(400} \mathrm{MHz,} \mathrm{CDCl}_{3}\right)$ of $\left(S_{a}, R\right)-\mathbf{1 1} \mathbf{b}$ :

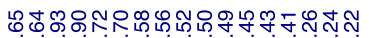

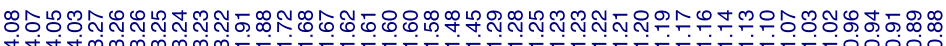

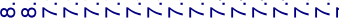

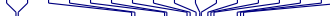

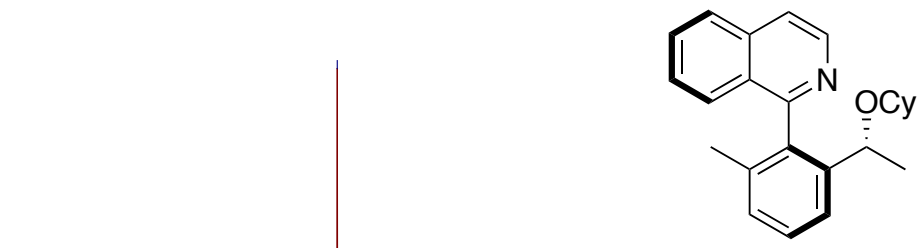

${ }^{13} \mathrm{NMR}\left(100 \mathrm{MHz}, \mathrm{CDCl}_{3}\right)$ of $\left(S_{a}, R\right)-\mathbf{1 1 b}$

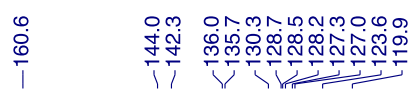

$\mid \begin{array}{lll}\infty & & 0 \\ & 0 \\ 1 & 0 \\ 1 & 1\end{array}$

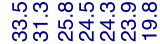

1)

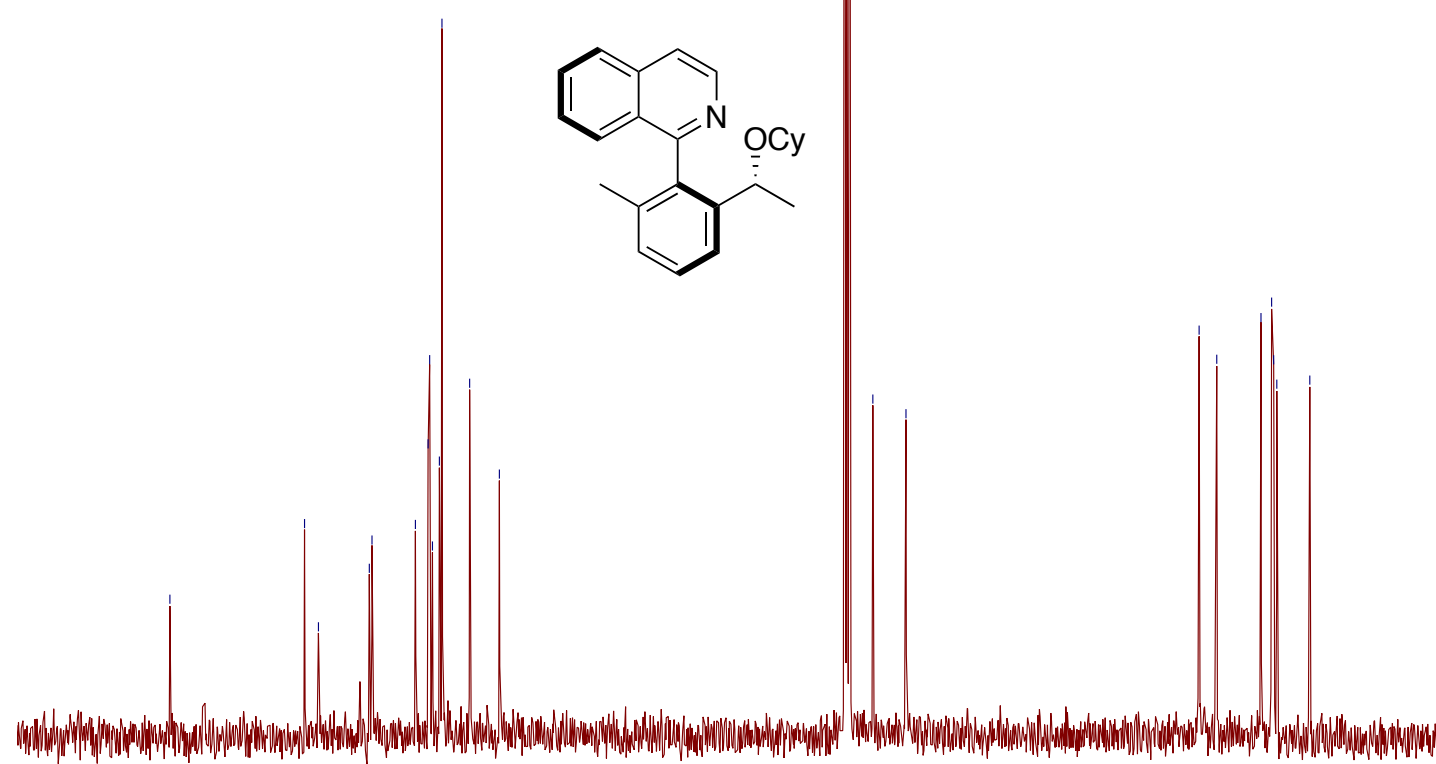


Racemic sample of $( \pm)$-11b: IA column, $n$-hexane:isopropanol 99:1, F= $0.5 \mathrm{~mL} / \mathrm{min}, \mathrm{T}=$ $30^{\circ} \mathrm{C}$.

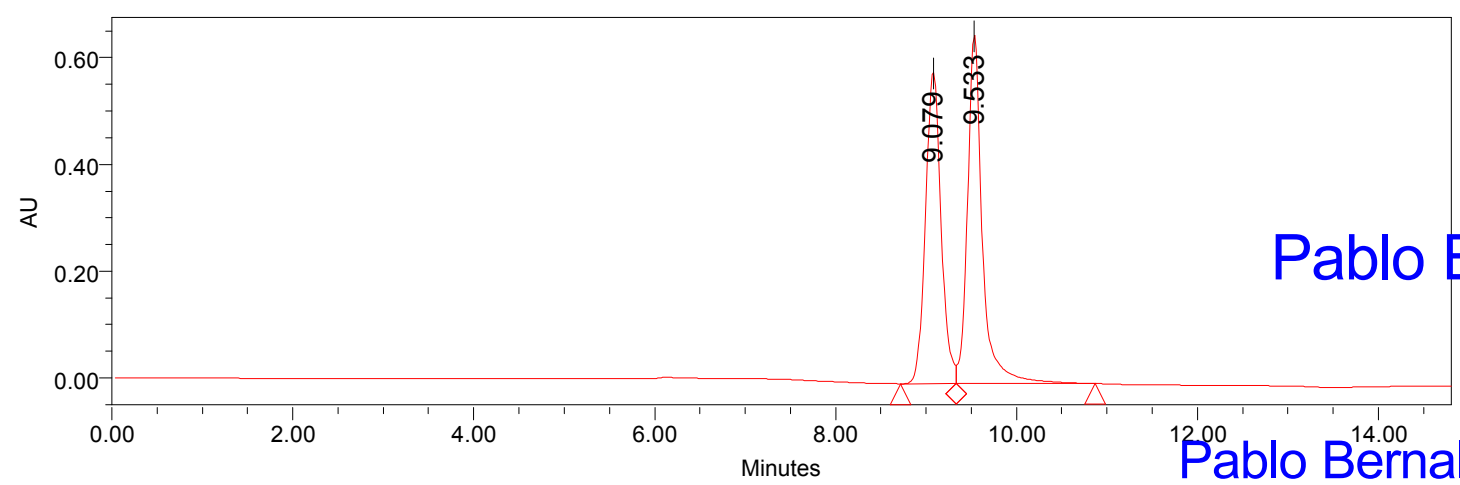

Processed Channel: PDA $220.0 \mathrm{~nm}$

\begin{tabular}{|r|l|r|r|r|r|}
\hline & Processed Channel & $\begin{array}{r}\text { Retention } \\
\text { Time }(\min )\end{array}$ & Area & $\%$ Area & Height \\
\hline 1 & PDA $220.0 \mathrm{~nm}$ & 9.079 & 6965826 & 48.31 & 584765 \\
\hline 2 & PDA $220.0 \mathrm{~nm}$ & 9.533 & 7453279 & 51.69 & 652822 \\
\hline
\end{tabular}

Enantioriched sample of $\left(S_{a}, R\right)-\mathbf{1 1} \mathbf{b}$ :

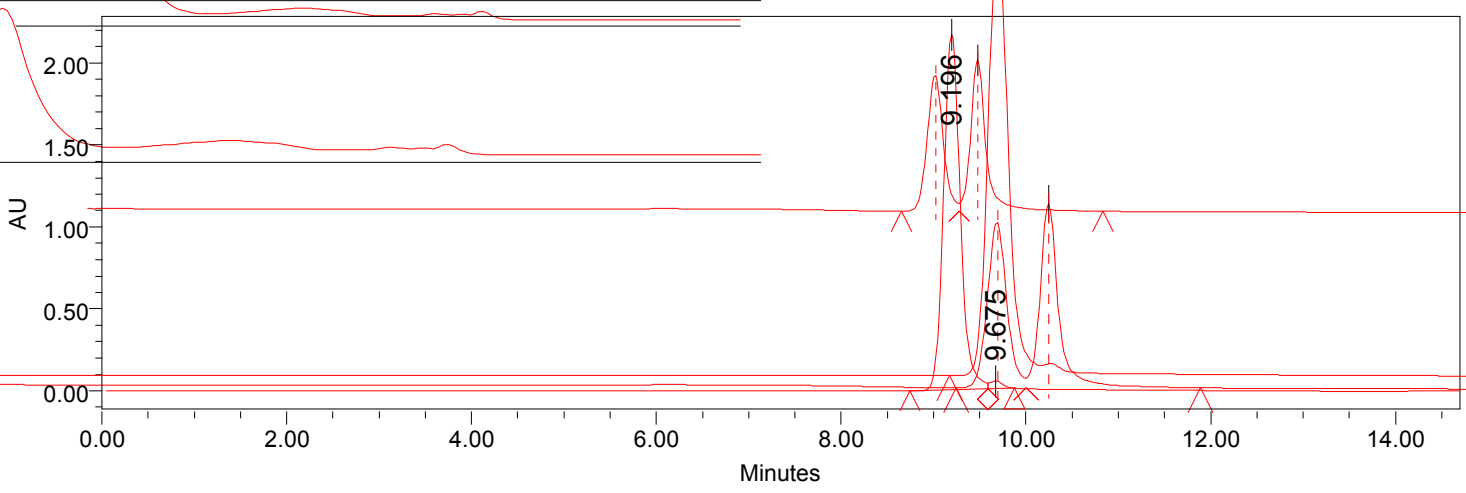

Processed Channel: PDA $218.0 \mathrm{~nm}$

\begin{tabular}{|r|l|r|r|r|r|}
\hline & Processed Channel & $\begin{array}{r}\text { Retention } \\
\text { Time }(\min )\end{array}$ & Area & \% Area & Height \\
\hline 1 & PDA 218.0 nm & 9.196 & 28846844 & 99.14 & 2252438 \\
\hline 2 & PDA 218.0 nm & 9.677 & 251306 & 0.86 & 45601 \\
\hline
\end{tabular}


${ }^{1} \mathrm{H}$ NMR $\left(400 \mathrm{MHz}, \mathrm{CDCl}_{3}\right)$ of $\left(S_{a}, R\right)-\mathbf{1 1 c}$ :

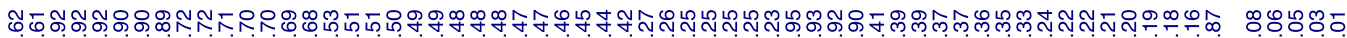

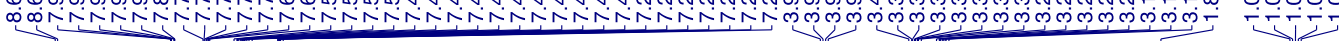<smiles>CCOC(C)c1cccc(C)c1-c1nccc2ccccc12</smiles>

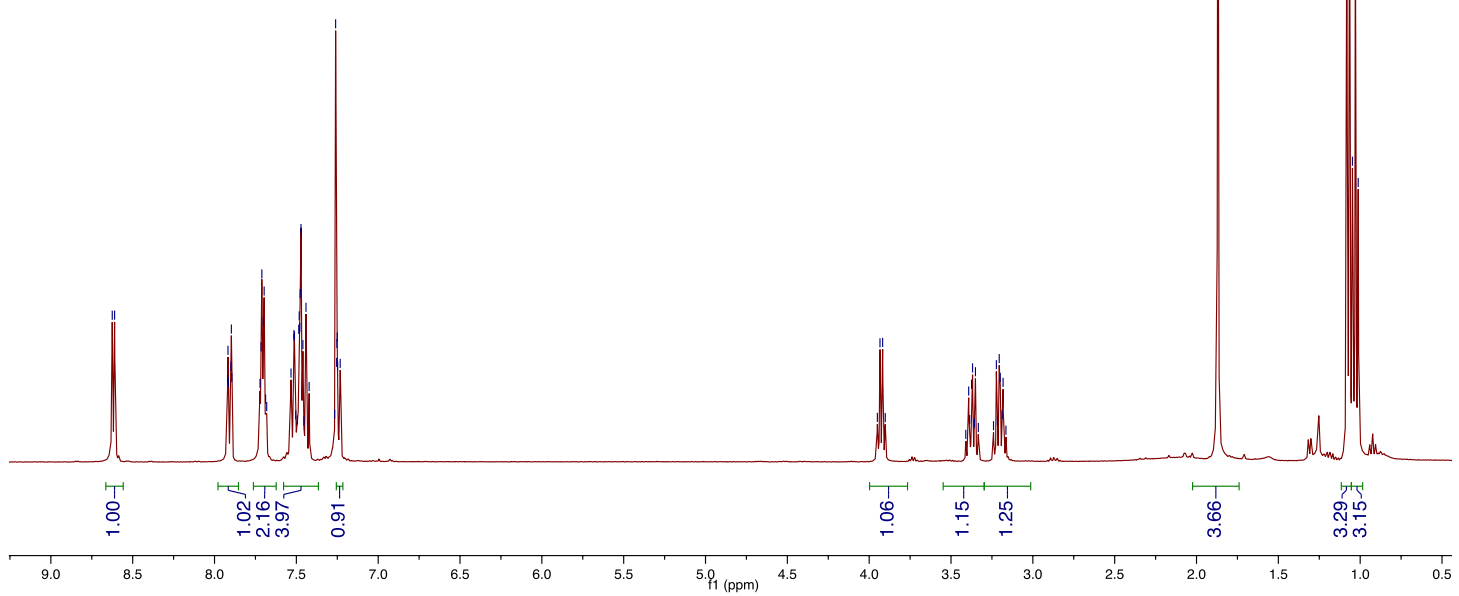

${ }^{13} \mathrm{C}$ NMR $\left(100 \mathrm{MHz}, \mathrm{CDCl}_{3}\right)$ of $\left(S_{a}, R\right)-11 \mathrm{c}$ :

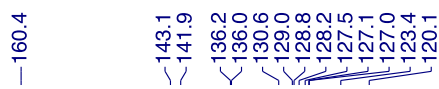

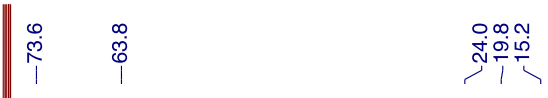

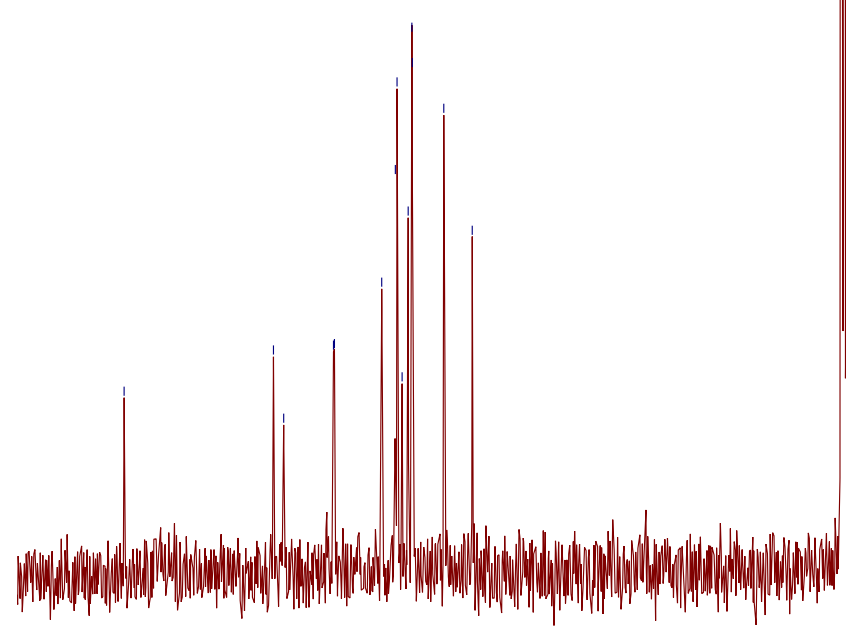<smiles>CCOC(C)c1cccc(C)c1-c1nccc2ccccc12</smiles> 
Racemic sample of $( \pm)$-11c: OD column, $n$-hexane:isopropanol 99:1, F= $1 \mathrm{~mL} / \mathrm{min}, \mathrm{T}=$ $30^{\circ} \mathrm{C}$.

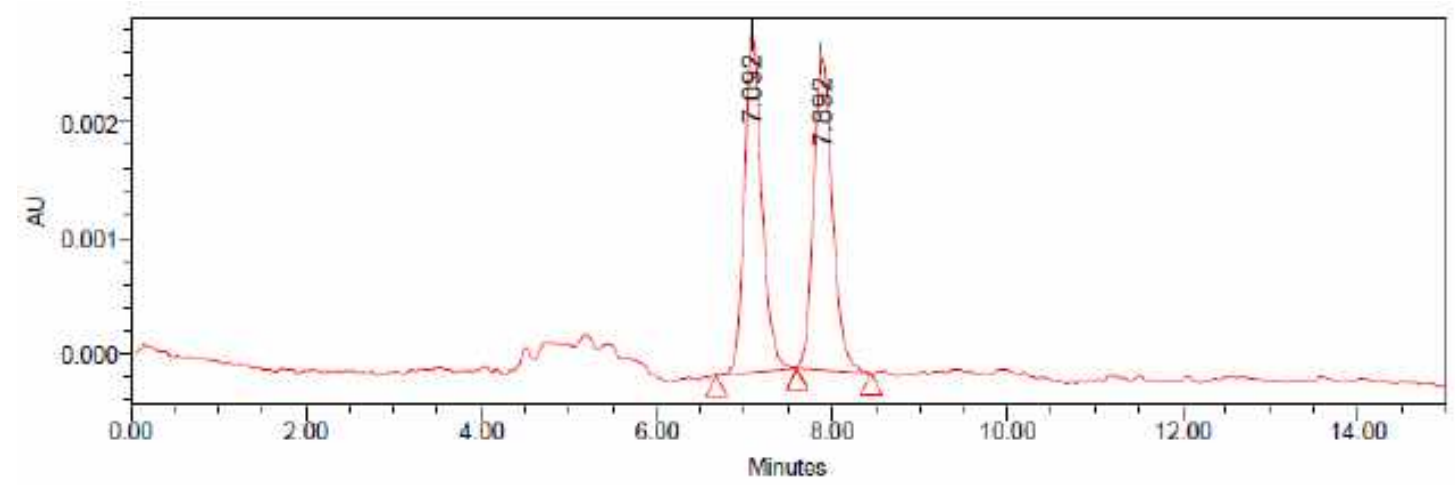

Processed Channel: PDA $269.0 \mathrm{~nm}$
\begin{tabular}{|l|l|r|r|r|r|}
\hline & Processed Channel & $\begin{array}{c}\text { Retention } \\
\text { Time }(\mathrm{min})\end{array}$ & Area & $\%$ Area & Height \\
\hline 1 & PDA $269.0 \mathrm{~nm}$ & 7.092 & 40305 & 49.72 & 2917 \\
\hline 2 & PDA $269.0 \mathrm{~nm}$ & 7.892 & 40751 & 50.28 & 2694 \\
\hline
\end{tabular}

Enantioriched sample of $\left(S_{a}, R\right)-11 \mathrm{c}$ :

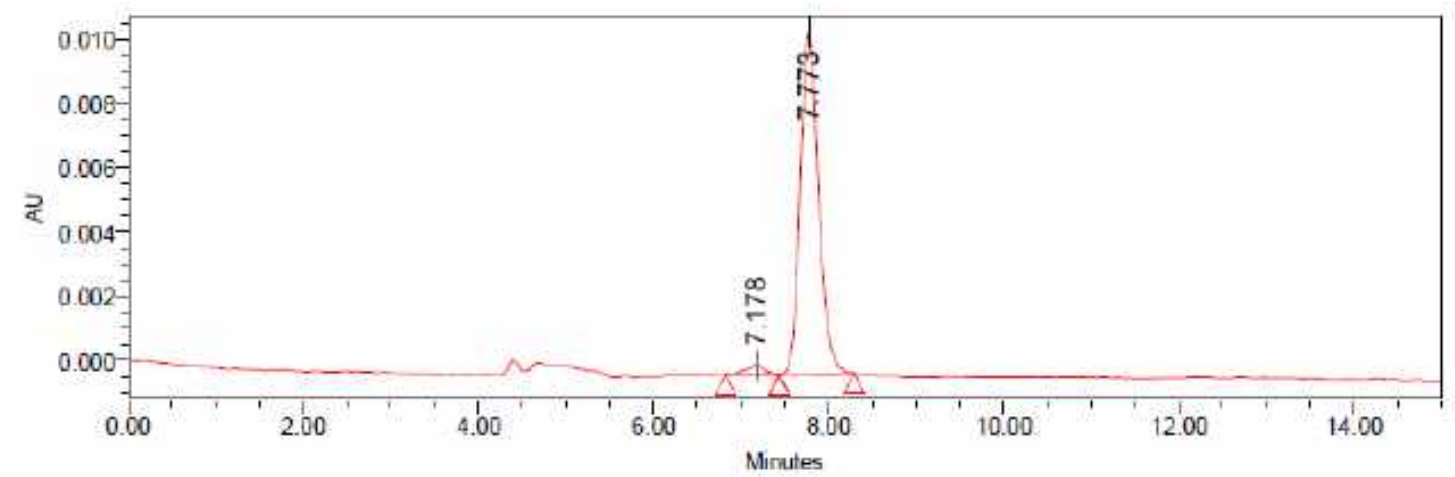

Processed Channel: PDA $269.0 \mathrm{~nm}$

\begin{tabular}{|r|l|r|r|r|r|}
\hline & Processed Channel & $\begin{array}{c}\text { Retention } \\
\text { Time }(\text { min) }\end{array}$ & Area & $\%$ Area & Height \\
\hline 1 & PDA 269.0 nm & 7.178 & 3925 & 2.50 & 256 \\
\hline 2 & PDA 269.0 nm & 7.773 & 153096 & 97.50 & 10600 \\
\hline
\end{tabular}


${ }^{1} \mathrm{H} \mathrm{NMR}\left(400 \mathrm{MHz}, \mathrm{CDCl}_{3}\right)$ of the minor diastereomer of $\mathbf{1 1 g}$ :

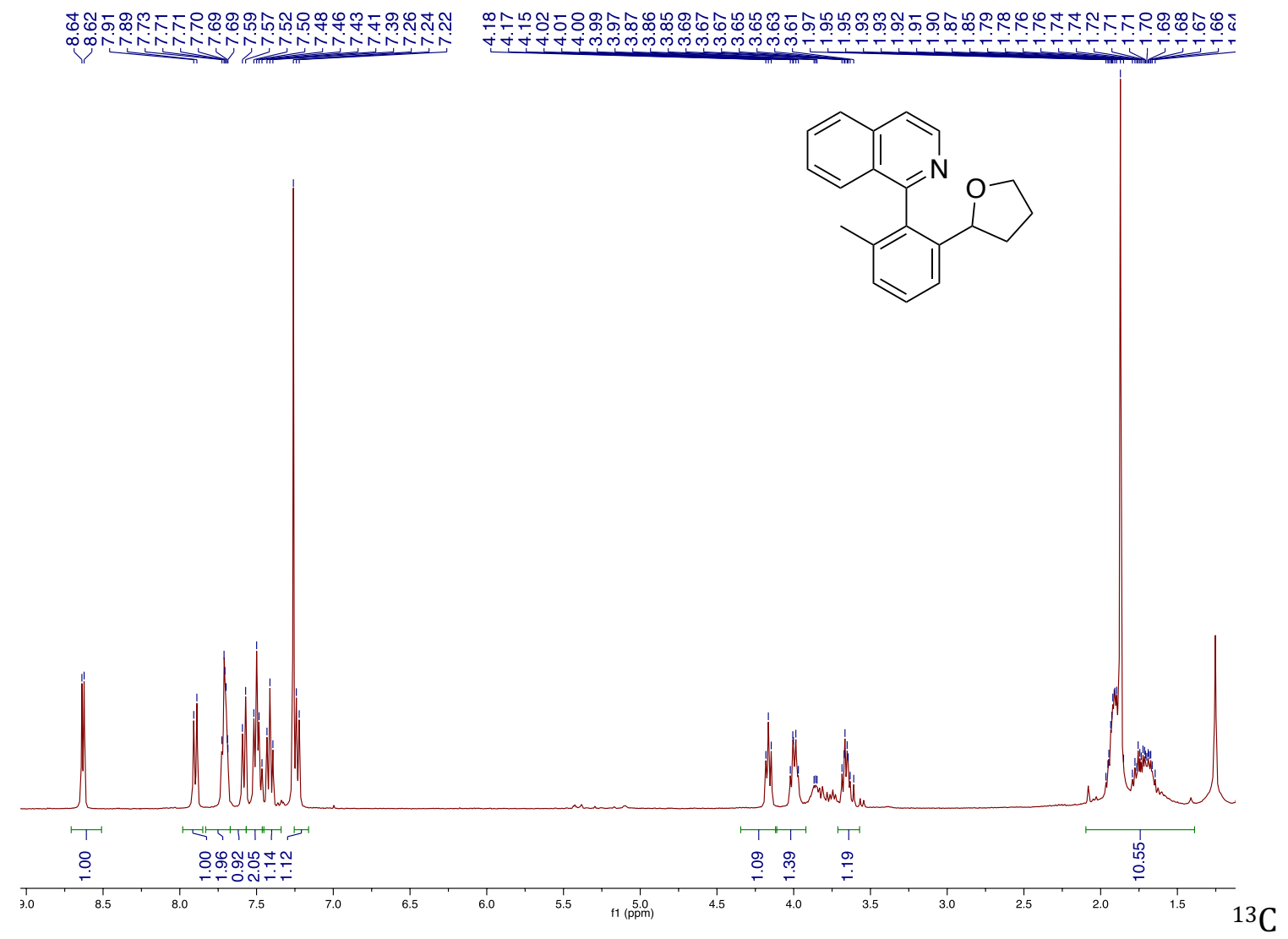

${ }^{13} \mathrm{C}-\mathrm{NMR}\left(100 \mathrm{MHz}, \mathrm{CDCl}_{3}\right)$ of the minor diastereomer of $\mathbf{1 1 g}$ :

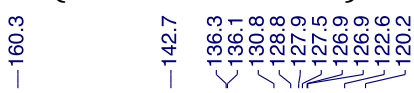

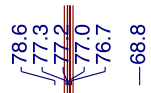

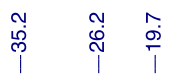<smiles>Cc1cccc(C2CCCO2)c1-c1nccc2ccccc12</smiles>

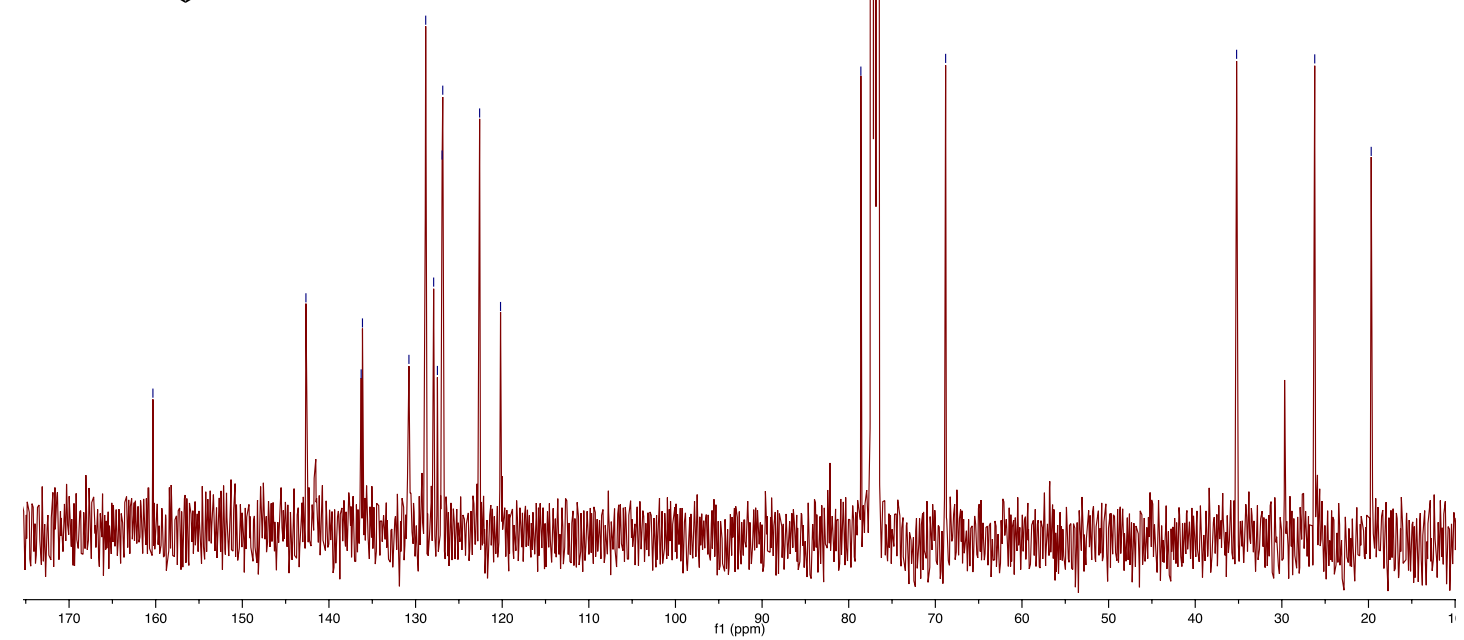


Racemic sample of the minor diastereomer of 11g: OD column, $n$ hexane:isopropanol 90:10, $\mathrm{F}=1 \mathrm{~mL} / \mathrm{min}, \mathrm{T}=30^{\circ} \mathrm{C}$.

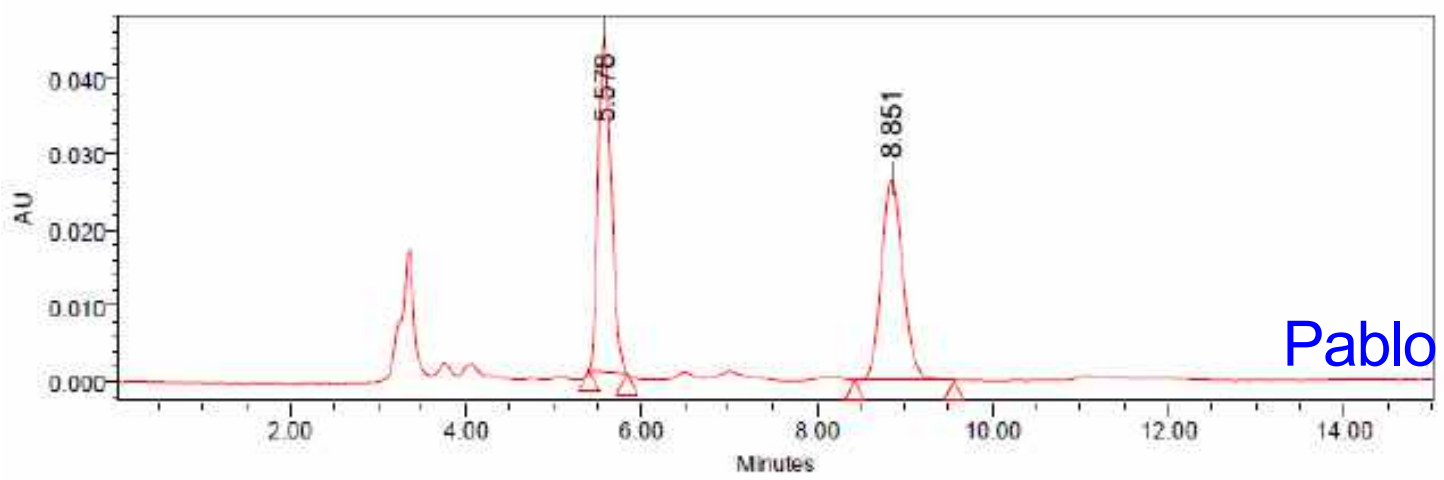

Processed Channel: PDA 217.9 nm
\begin{tabular}{|l|l|r|r|r|r|}
\hline & Processed Channel & $\begin{array}{l}\text { Retention } \\
\text { Time }(\mathrm{min})\end{array}$ & Area & $\%$ Area & Height \\
\hline 1 & PDA $217.9 \mathrm{~nm}$ & 5.578 & 458094 & 50.43 & 44638 \\
\hline 2 & PDA $217.9 \mathrm{~nm}$ & 8.851 & 450339 & 49.57 & 26567 \\
\hline
\end{tabular}

\section{Enantioriched sample of the minor diastereomer of 11g:}

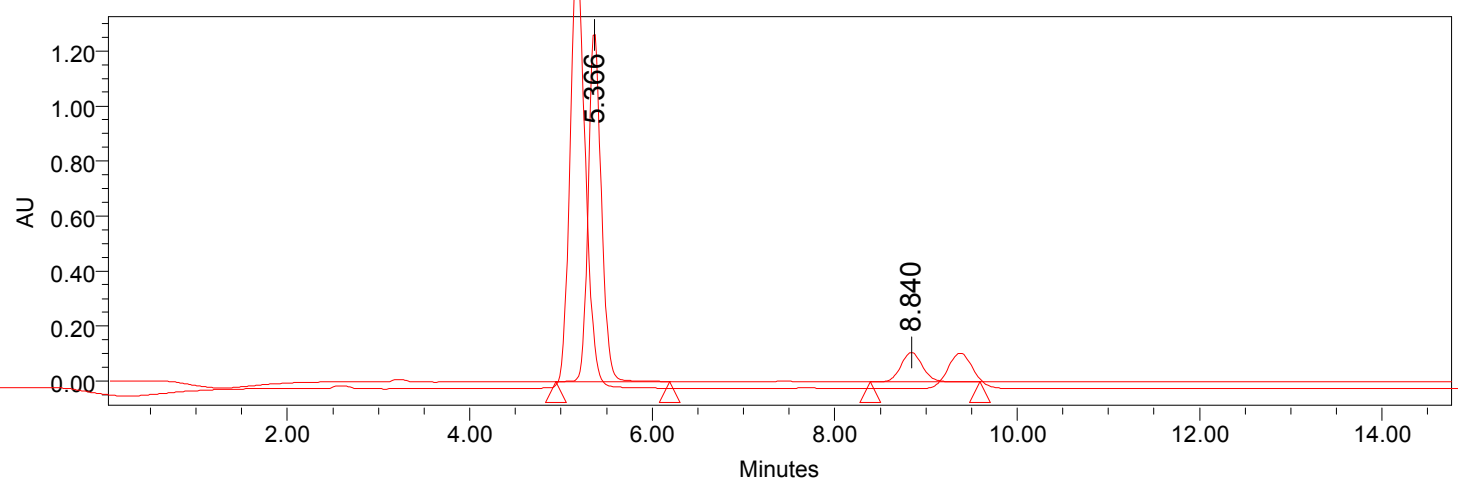

\section{Processed Channel: PDA 217.9 nm}

\begin{tabular}{|r|l|r|r|r|r|}
\hline & Processed Channel & $\begin{array}{r}\text { Retention } \\
\text { Time }(\min )\end{array}$ & Area & \% Area & Height \\
\hline 1 & PDA 217.9 nm & 5.366 & 12573286 & 87.79 & 1267600 \\
\hline 2 & PDA 217.9 nm & 8.840 & 1749095 & 12.21 & 107183 \\
\hline
\end{tabular}


${ }^{1} \mathrm{H}$ NMR $\left(400 \mathrm{MHz}, \mathrm{CDCl}_{3}\right)$ of $\left(R_{a}, S\right) \mathbf{- 1 1 g}$ :

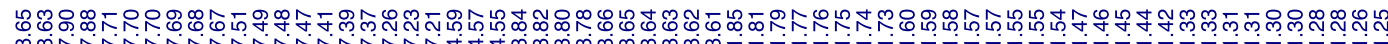

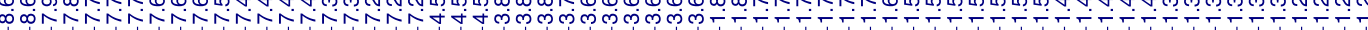<smiles>Cc1cccc(C2CCCO2)c1-c1nccc2ccccc12</smiles>

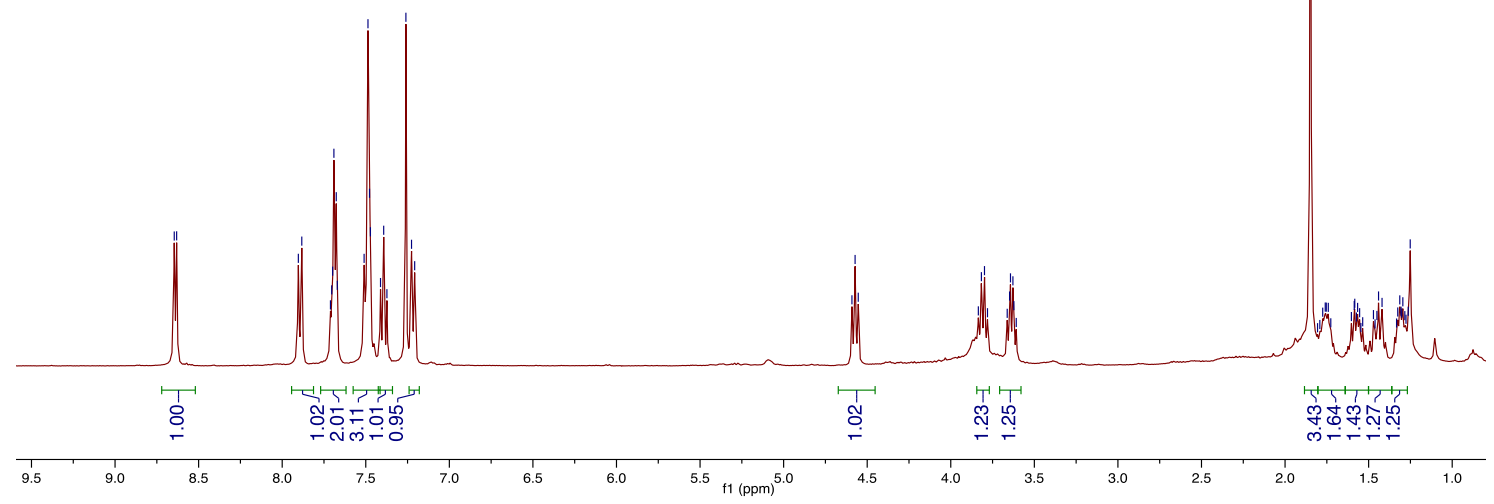

${ }^{13} \mathrm{C}$ NMR $\left(100 \mathrm{MHz}, \mathrm{CDCl}_{3}\right)$ of $\left(R_{a}, S\right)-\mathbf{1 1 g}$ :
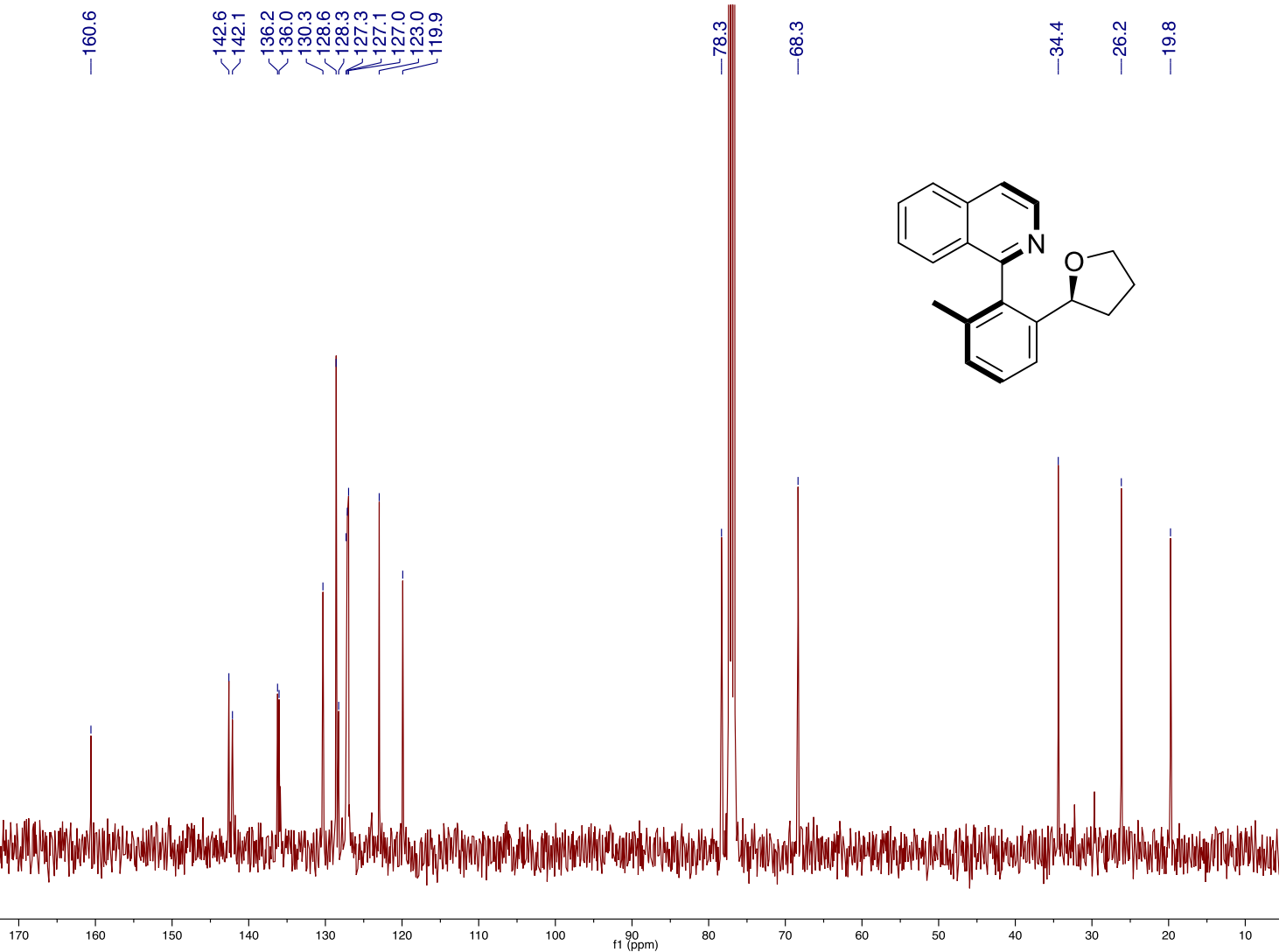
Racemic sample of the major diastereomer of $( \pm)-11 g:$ IA column, $n$ hexane:isopropanol $90: 10, \mathrm{~F}=1 \mathrm{~mL} / \mathrm{min}, \mathrm{T}=30^{\circ} \mathrm{C}$

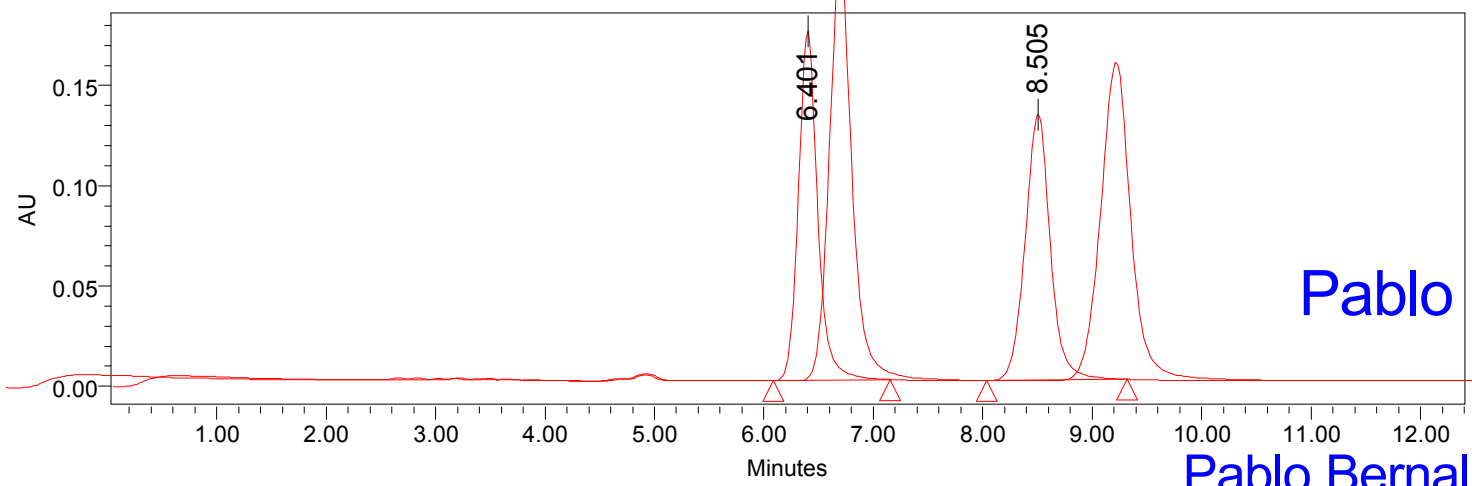

\section{Processed Channel: PDA $218.0 \mathrm{~nm}$}

\begin{tabular}{|r|l|r|c|r|r|}
\hline & Processed Channel & $\begin{array}{c}\text { Retention } \\
\text { Time }(\mathrm{min})\end{array}$ & \multicolumn{1}{|c|}{ Area } & \% Area & Height \\
\hline 1 & PDA 218.0 nm & 6.401 & 2128395 & 50.02 & 174232 \\
\hline 2 & PDA 218.0 nm & 8.505 & 2126695 & 49.98 & 132803 \\
\hline
\end{tabular}

Enantioriched sample of the major diatereomer of $\left(R_{a}, S\right)-11 g$ :

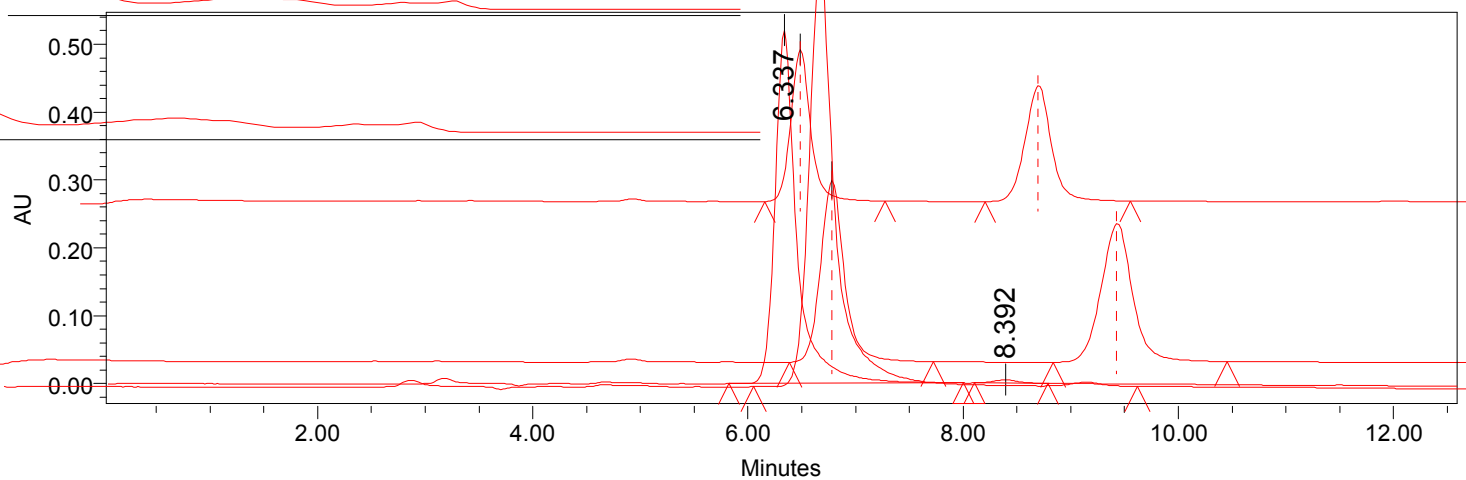

Processed Channel: PDA 217.9 nm

\begin{tabular}{|r|l|r|r|r|r|}
\hline & Processed Channel & $\begin{array}{r}\text { Retention } \\
\text { Time (min) }\end{array}$ & Area & \% Area & Height \\
\hline 1 & PDA 217.9 nm & 6.337 & 7411813 & 99.00 & 520011 \\
\hline 2 & PDA 217.9 nm & 8.392 & 74976 & 1.00 & 4880 \\
\hline
\end{tabular}


${ }^{1} \mathrm{H}$ NMR $\left(400 \mathrm{MHz}, \mathrm{CDCl}_{3}\right)$ of $\left(S_{a}, R\right)-\mathbf{1 9}$ :

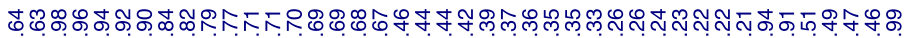

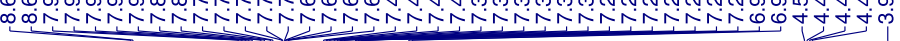<smiles>CC(O)c1ccc2ccccc2c1-c1nccc2ccccc12</smiles>

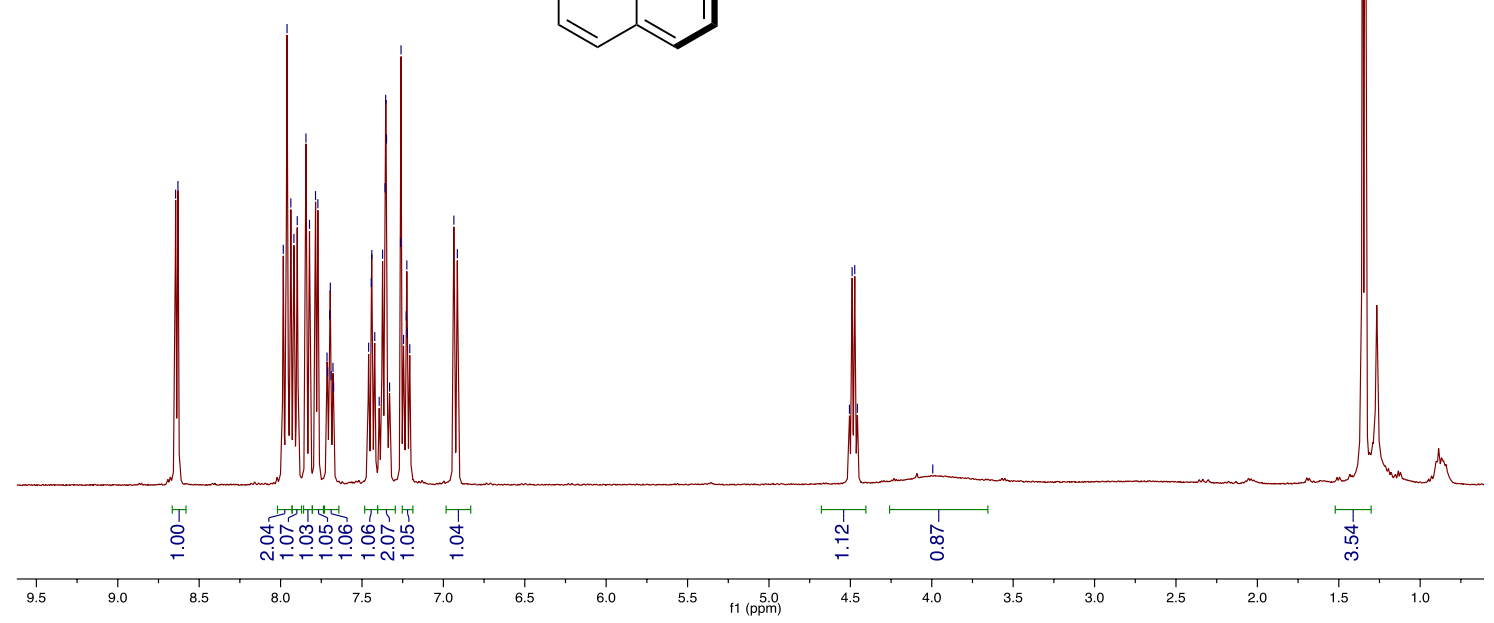

${ }^{13} \mathrm{C}$ NMR $\left(100 \mathrm{MHz}, \mathrm{CDCl}_{3}\right)$ of $\left(S_{a}, R\right)-\mathbf{1 9}$ :

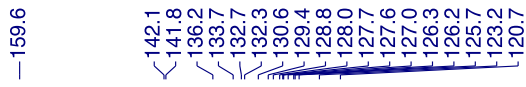

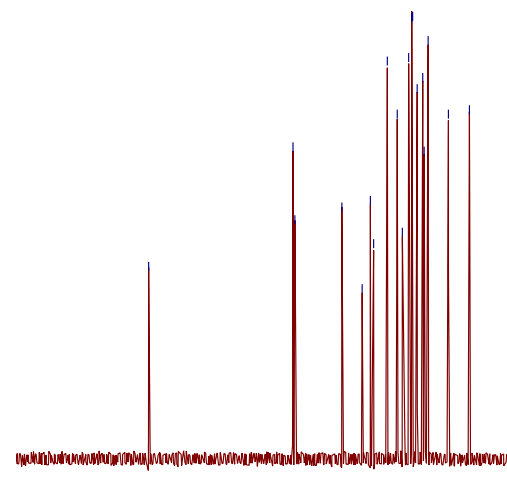<smiles>C[C@H](O)c1ccc2ccccc2c1-c1nccc2ccccc12</smiles>

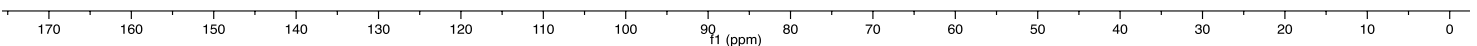


Racemic sample of $( \pm)$-19: AS-H column, $n$-hexane:isopropanol 85:15, $\mathrm{F}=1 \mathrm{~mL} / \mathrm{min}$, $\mathrm{T}=30^{\circ} \mathrm{C}$.

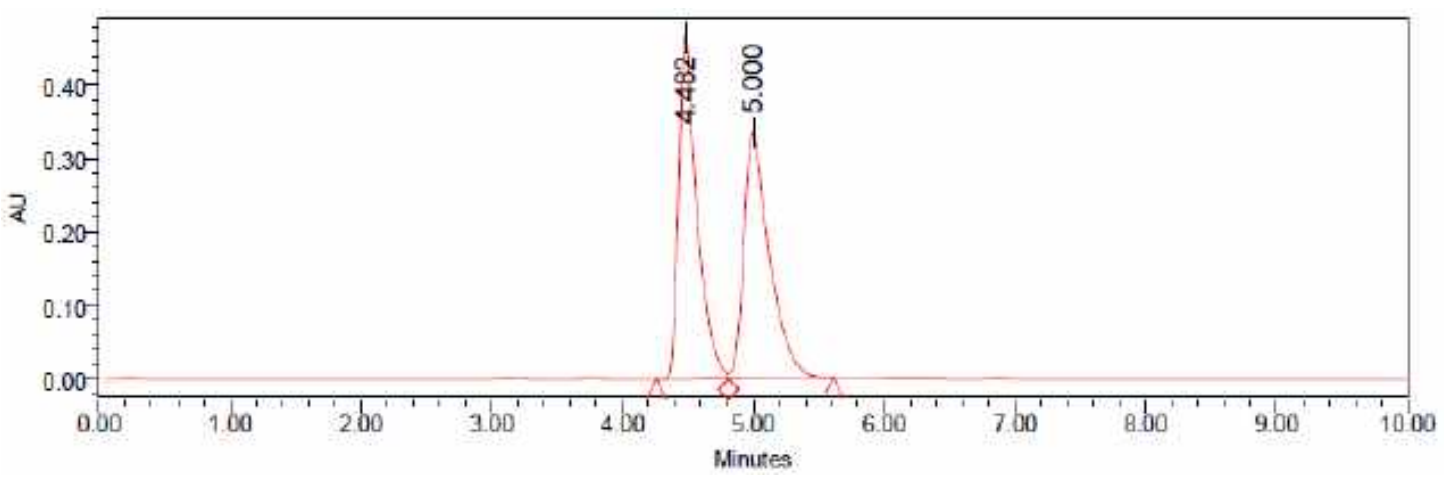

Processed Channel: PDA $230.1 \mathrm{~nm}$

\begin{tabular}{|l|l|r|r|r|r|}
\hline & Processed Channel & $\begin{array}{r}\text { Retention } \\
\text { Time }(\mathrm{min})\end{array}$ & Area & \% Area & Height \\
\hline 1 & PDA 230.1 nm & 4.482 & 4528417 & 49.73 & 467120 \\
\hline 2 & PDA $230.1 \mathrm{~nm}$ & 5.000 & 4578131 & 50.27 & 335309 \\
\hline
\end{tabular}

\section{Enantioriched sample of $\left(S_{a}, R\right)-19$ :}

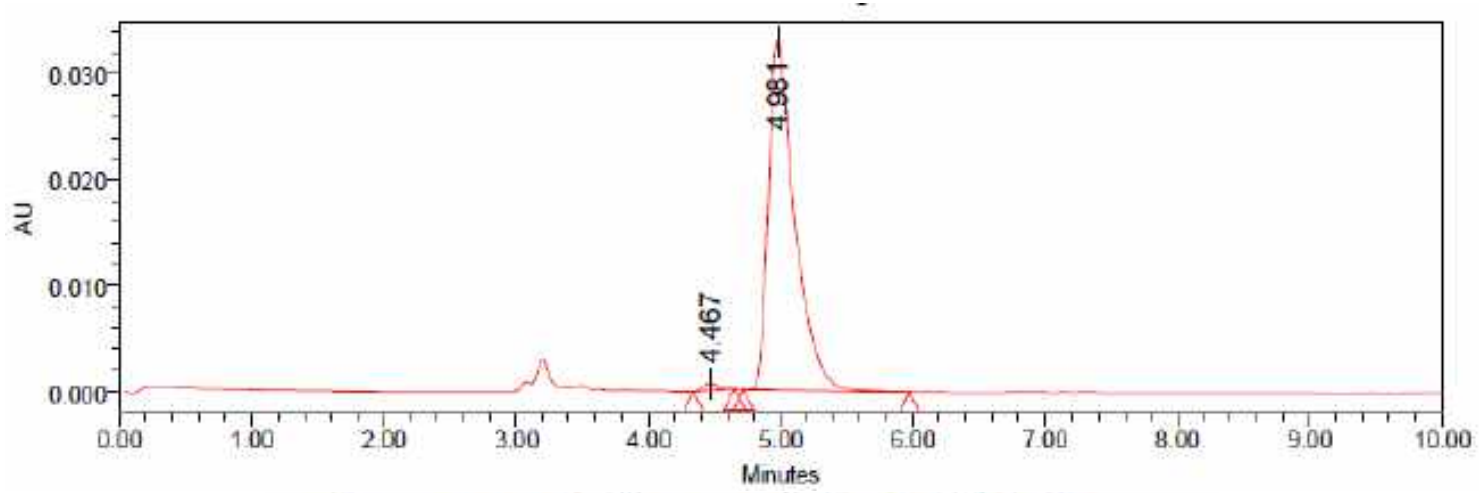

\section{Processed Channel: PDA $230.1 \mathrm{~nm}$}

\begin{tabular}{|l|l|r|r|r|r|}
\hline & Processed Channel & $\begin{array}{r}\text { Retention } \\
\text { Time (min) }\end{array}$ & Area & \% Area & Height \\
\hline 1 & PDA 230.1 nm & 4.467 & 5545 & 1.20 & 671 \\
\hline 2 & PDA 230.1 nm & 4.981 & 455155 & 98.80 & 33161 \\
\hline
\end{tabular}


${ }^{1} \mathrm{H}$ NMR $\left(400 \mathrm{MHz}, \mathrm{CDCl}_{3}\right)$ of $\left(S_{a}, R\right)-\mathbf{2 0}$ :

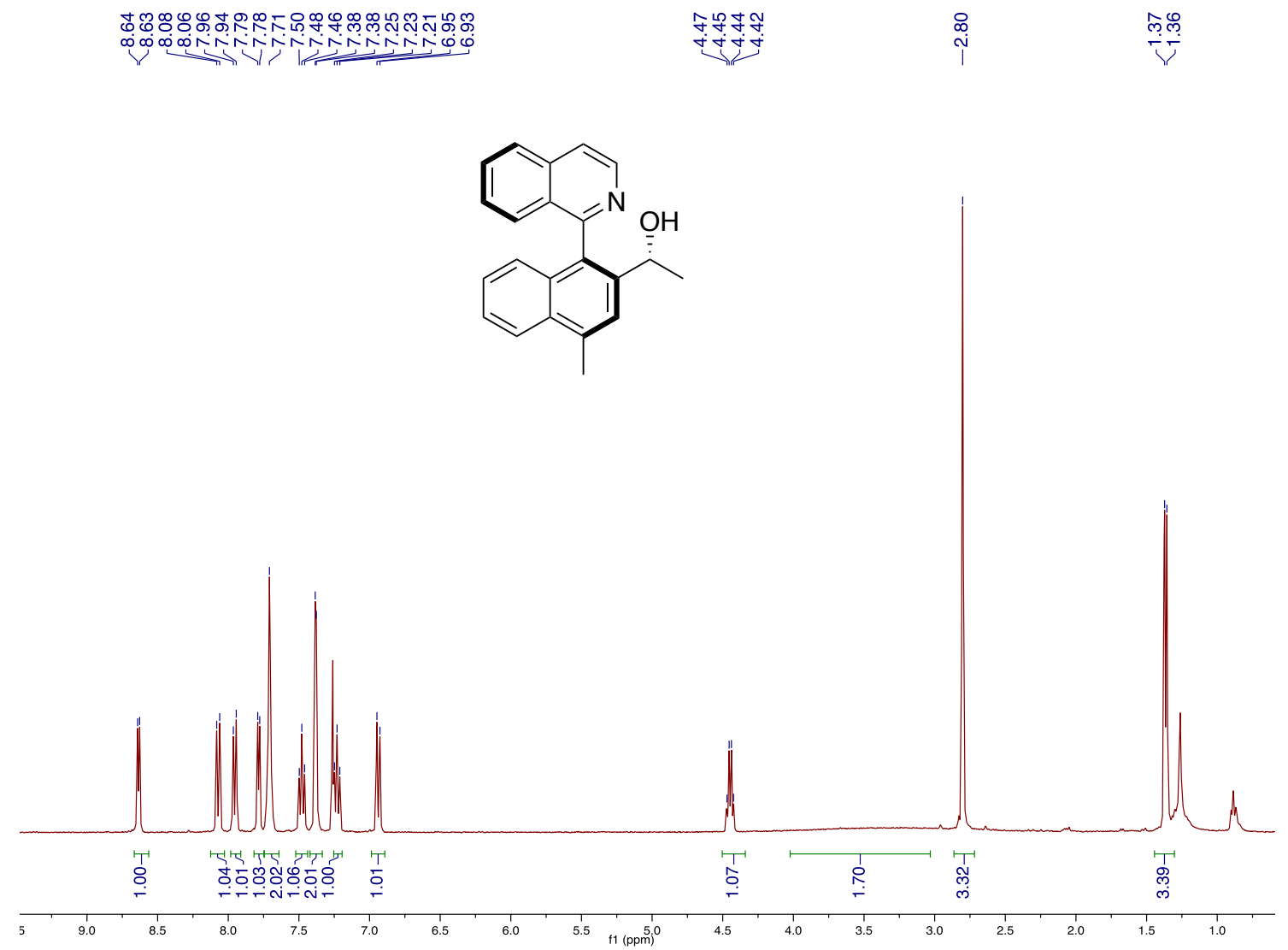

${ }^{13} \mathrm{C}$ NMR $\left(100 \mathrm{MHz}, \mathrm{CDCl}_{3}\right)$ of $\left(S_{a} R\right)-\mathbf{2 0}$ :
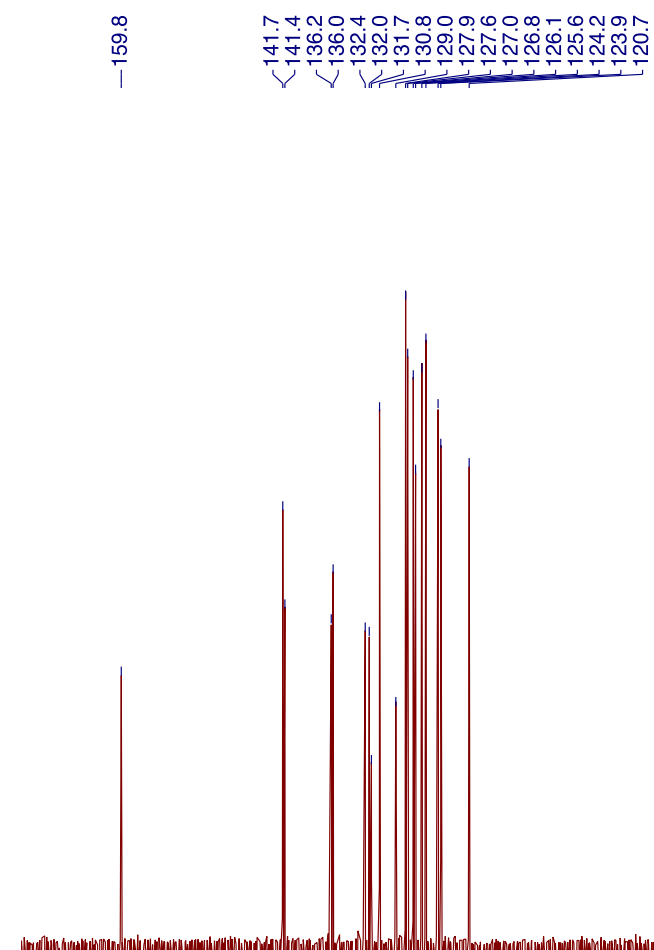

@̊

$\stackrel{\infty}{i} \underset{i}{\infty}$<smiles>Cc1cc([C@H](C)O)c(-c2nccc3ccccc23)c2ccccc12</smiles>

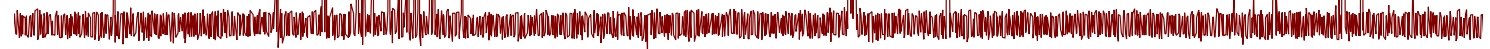

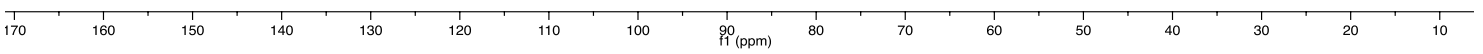


Racemic sample of $( \pm)$-20: IA column, $n$-hexane:isopropanol 90:10, F= $1 \mathrm{~mL} / \mathrm{min}, \mathrm{T}=$ $30^{\circ} \mathrm{C}$.

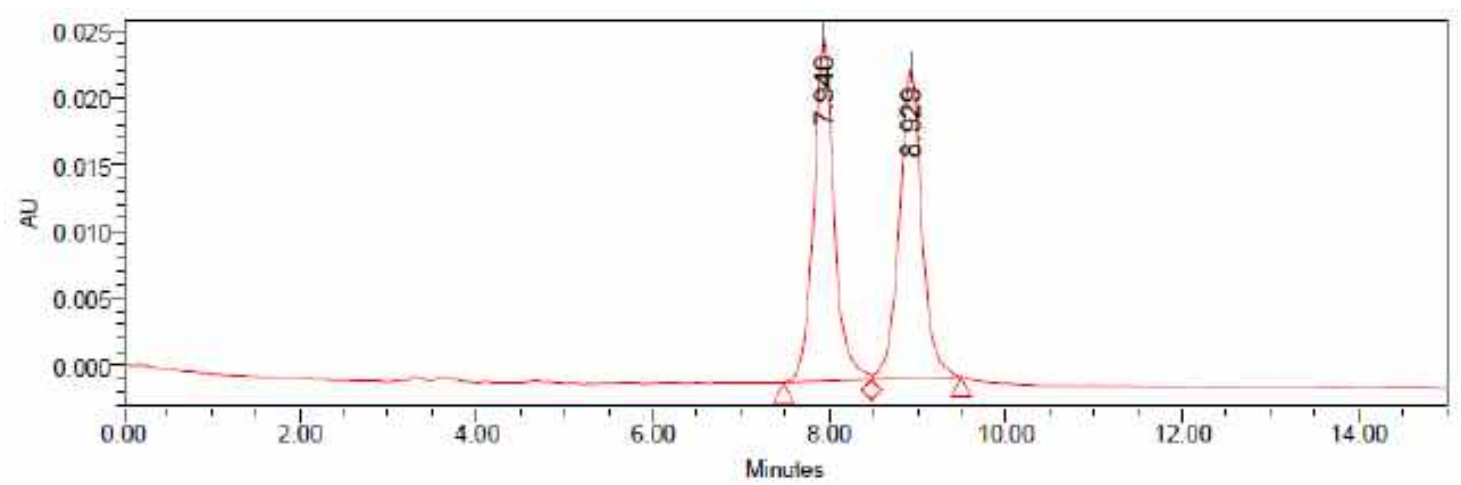

Processed Channel: PDA 221.0 nm
\begin{tabular}{|l|l|r|r|r|r|}
\hline & Processed Channel & $\begin{array}{c}\text { Retention } \\
\text { Time }(\mathrm{min})\end{array}$ & Area & $\%$ Area & Height \\
\hline 1 & PDA $221.0 \mathrm{~nm}$ & 7.940 & 415251 & 50.43 & 25735 \\
\hline 2 & PDA $221.0 \mathrm{~nm}$ & 8.929 & 408152 & 49.57 & 23233 \\
\hline
\end{tabular}

Enantioriched sample of $\left(S_{a}, R\right)$-20:

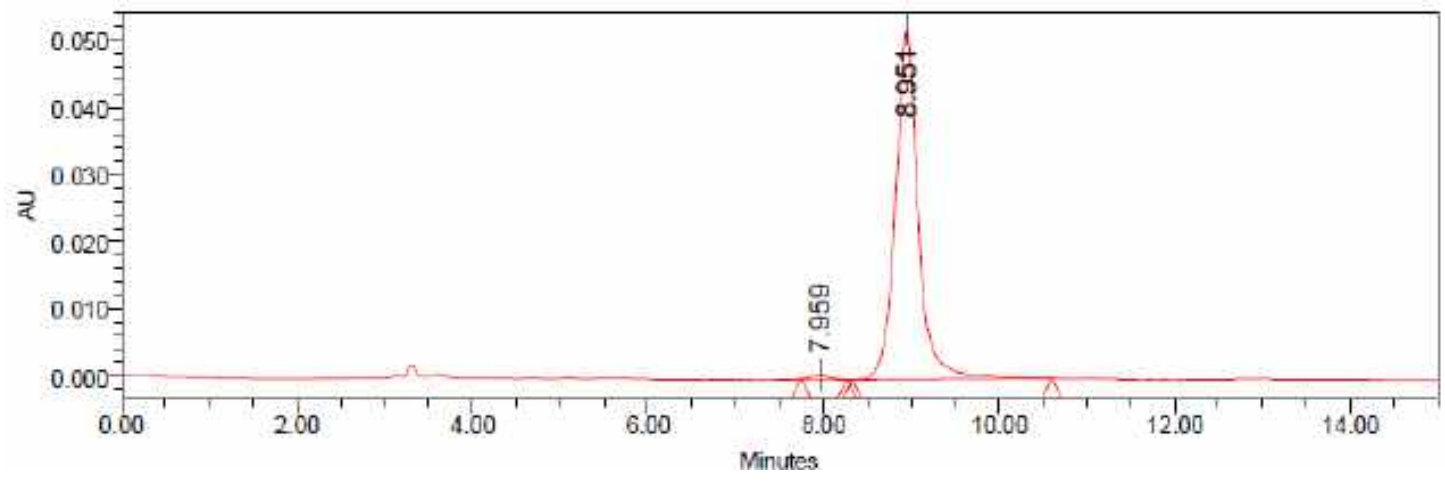

Processed Channel: PDA $221.0 \mathrm{~nm}$

\begin{tabular}{|r|l|r|r|r|r|}
\hline & Processed Channel & $\begin{array}{r}\text { Retention } \\
\text { Time }(\mathrm{min})\end{array}$ & Area & \% Area & Height \\
\hline 1 & PDA 221.0 nm & 7.959 & 9110 & 0.96 & 690 \\
\hline 2 & PDA $221.0 \mathrm{~nm}$ & 8.951 & 937485 & 99.04 & 52094 \\
\hline
\end{tabular}


${ }^{1} \mathrm{H}$ NMR $\left(400 \mathrm{MHz}, \mathrm{CDCl}_{3}\right)$ of $\left(S_{a}, R\right)-\mathbf{2 1}$ :

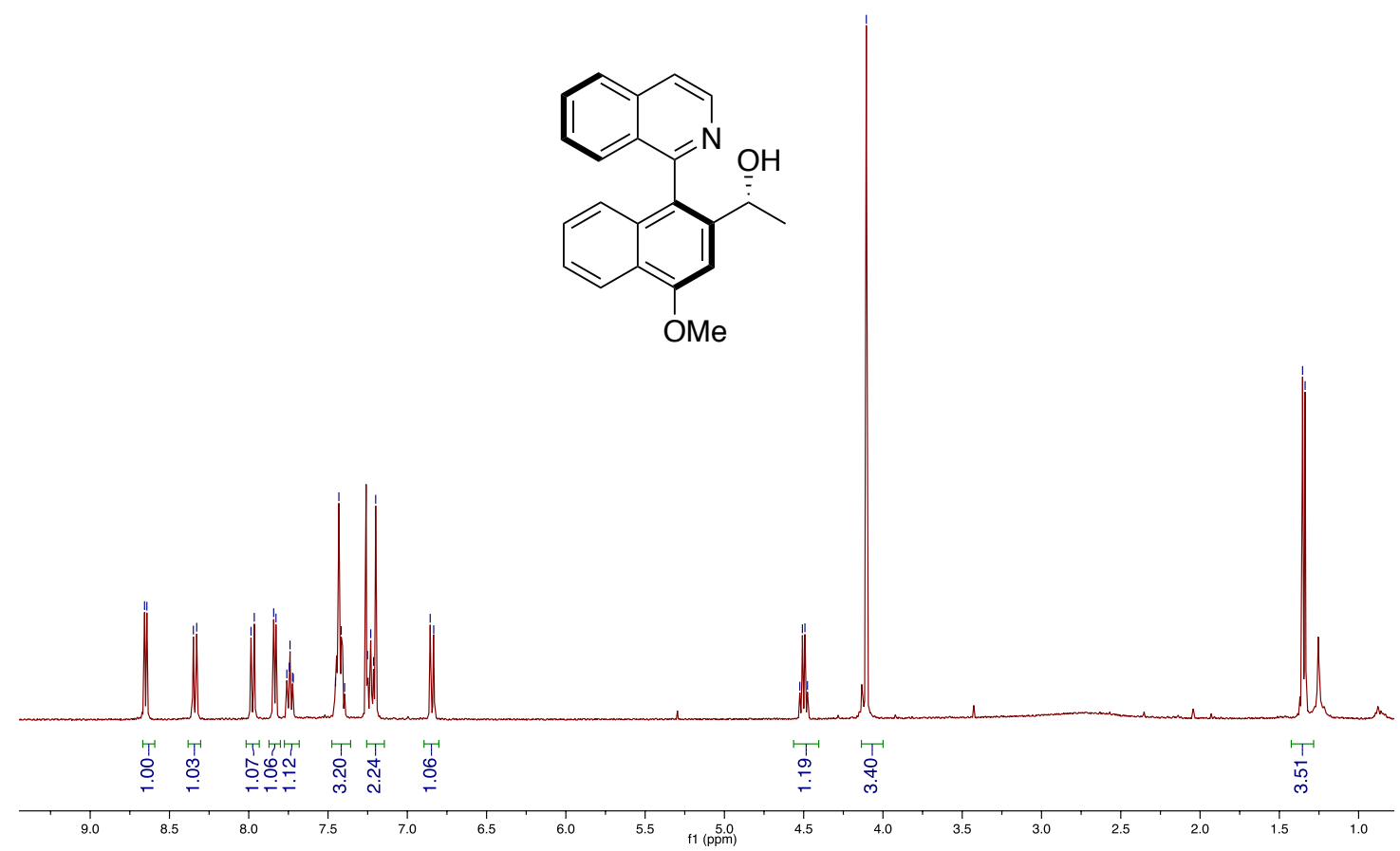

${ }^{13} \mathrm{C}$ NMR (100 MHz, $\left.\mathrm{CDCl}_{3}\right)$ of $\left(S_{a}, R\right)-\mathbf{2 1}$ :

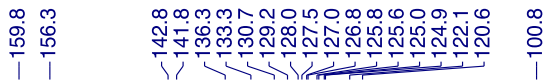

\section{@̊}<smiles>COc1cc([C@H](C)O)c(-c2nccc3ccccc23)c2ccccc12</smiles>

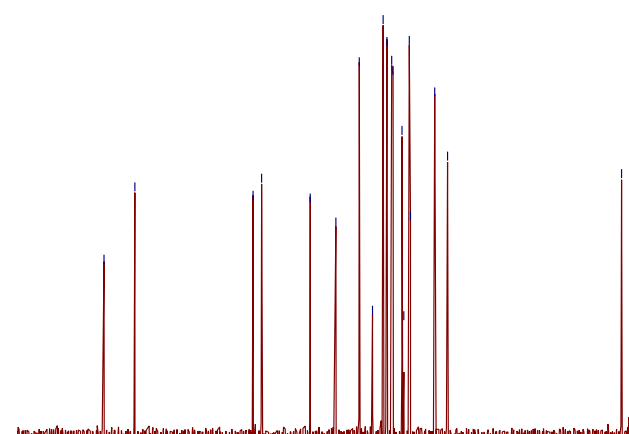


Racemic sample of $( \pm)$-21: IA column, $n$-hexane:isopropanol 90:10, F= $1 \mathrm{~mL} / \mathrm{min}, \mathrm{T}=$ $30^{\circ} \mathrm{C}$.

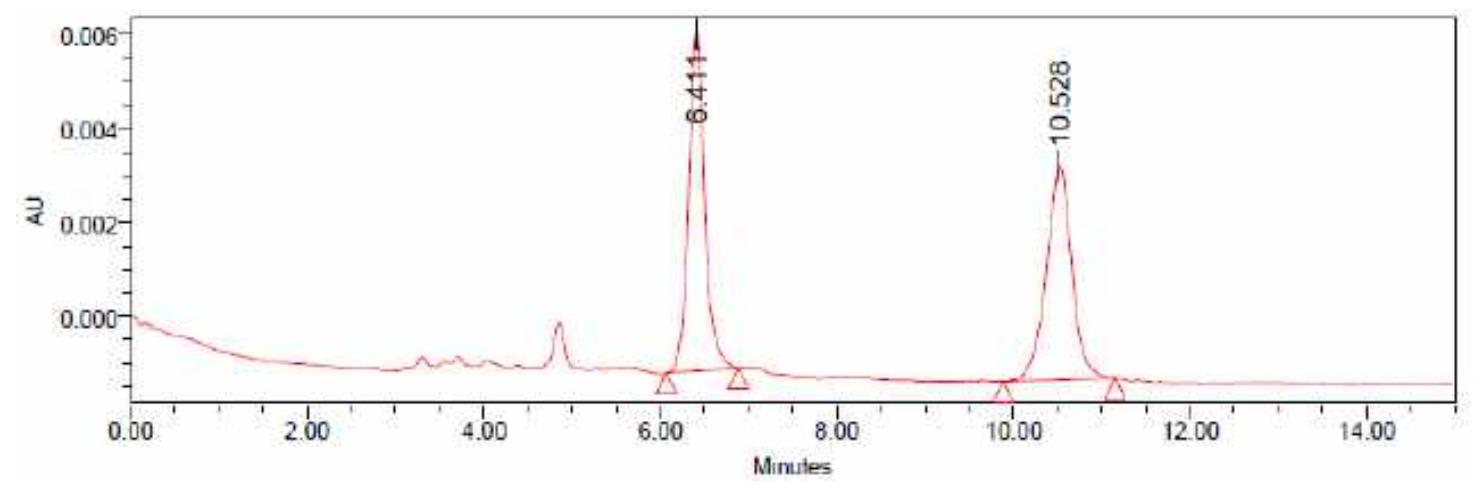

\section{Processed Channel: PDA $240.0 \mathrm{~nm}$}

\begin{tabular}{|r|l|r|r|r|r|}
\hline & Processed Channel & $\begin{array}{r}\text { Retention } \\
\text { Time (min) }\end{array}$ & Area & \% Area & Height \\
\hline 1 & PDA 240.0 nm & 6.411 & 91278 & 50.00 & 7179 \\
\hline 2 & PDA $240.0 \mathrm{~nm}$ & 10.528 & 91291 & 50.00 & 4552 \\
\hline
\end{tabular}

Enantioriched sample of $\left(S_{a}, R\right)-21$ :

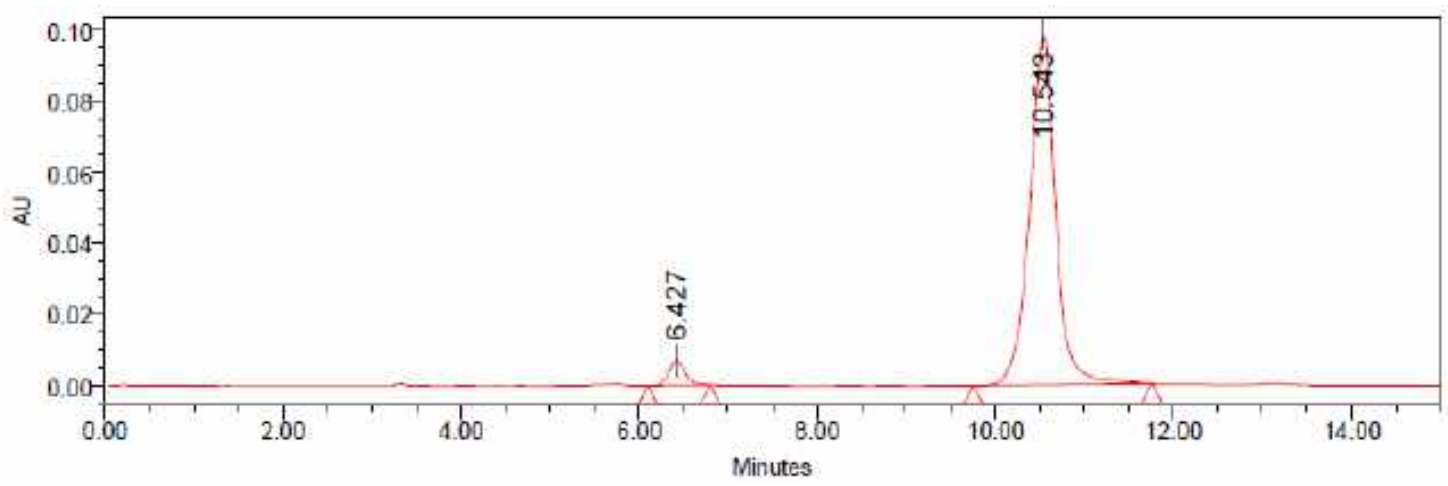

\section{Processed Channel: PDA $240.0 \mathrm{~nm}$}

\begin{tabular}{|r|l|r|r|r|r|}
\hline & Processed Channel & $\begin{array}{c}\text { Retention } \\
\text { Time (min) }\end{array}$ & Area & \% Area & Height \\
\hline 1 & PDA 240.0 nm & 6.427 & 85961 & 4.11 & 7007 \\
\hline 2 & PDA 240.0 nm & 10.543 & 2005005 & 95.89 & 98523 \\
\hline
\end{tabular}


${ }^{1} \mathrm{H}$ NMR $\left(400 \mathrm{MHz}, \mathrm{CDCl}_{3}\right)$ of $\left(S_{a}, R\right)-22$ :

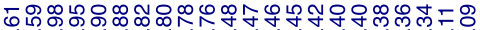

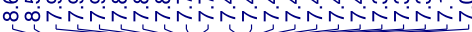

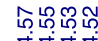

ن்

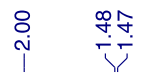

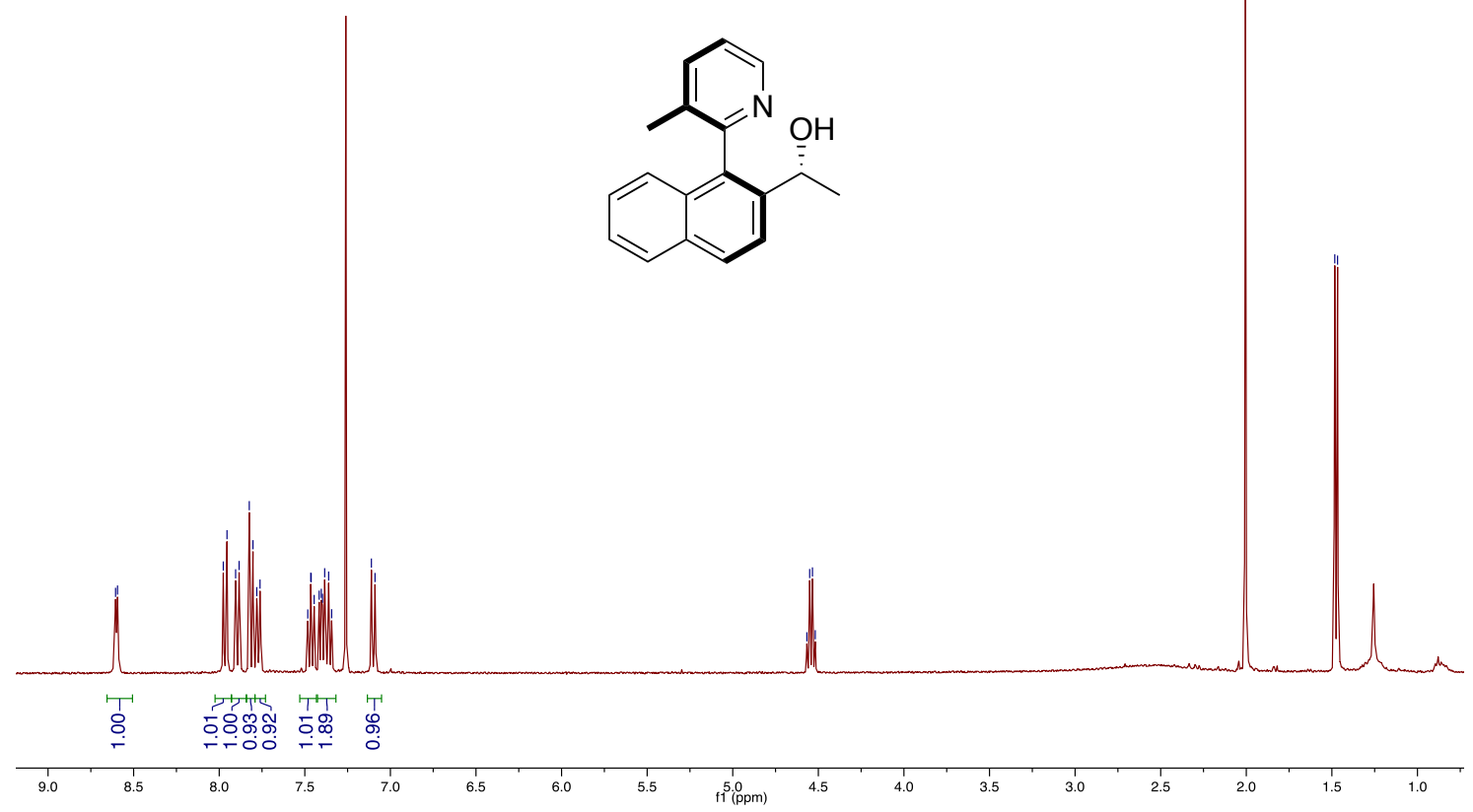

${ }^{13} \mathrm{C}$ NMR $\left(100 \mathrm{MHz}, \mathrm{CDCl}_{3}\right)$ of $\left(S_{a}, R\right)-22$ :

г.

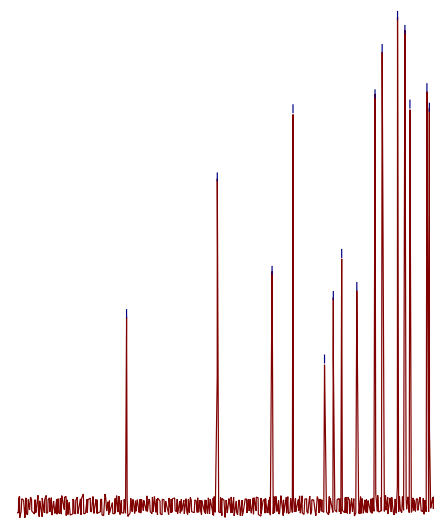

$m$
0
0
1

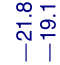

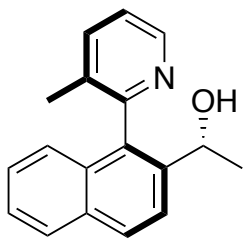

$1_{100}+1,150$

10

${ }^{90} 0_{11(\mathrm{ppm})} 80$ 
Racemic sample of $( \pm)$-15: AS-H column, $n$-hexane:isopropanol 90:10, $\mathrm{F}=1 \mathrm{~mL} / \mathrm{min}$, $\mathrm{T}=30^{\circ} \mathrm{C}$.

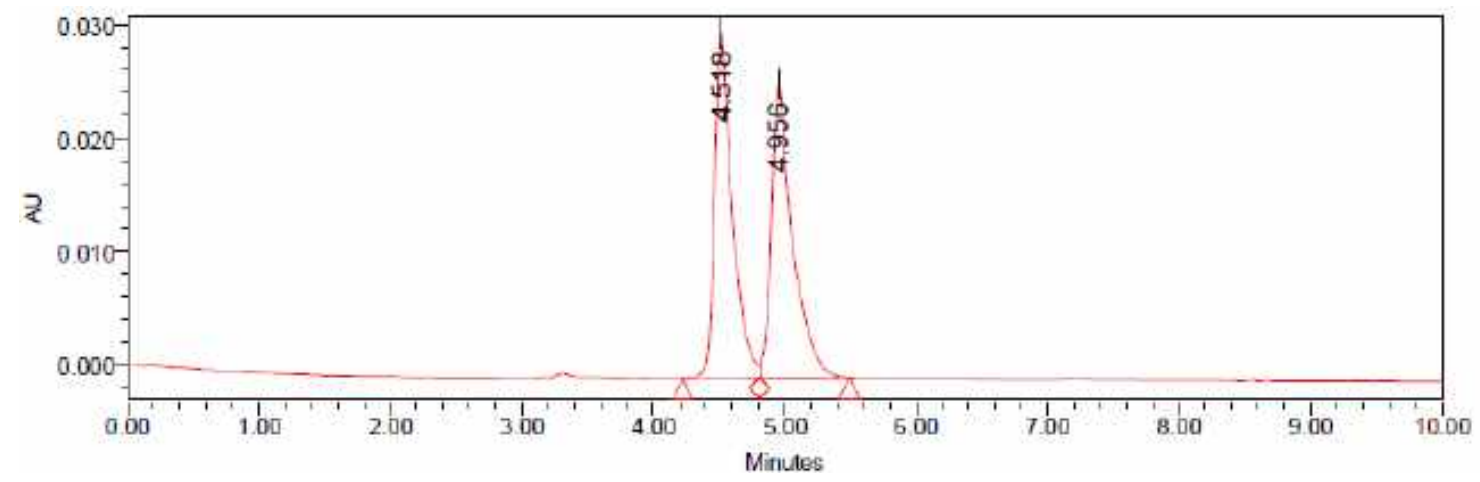

\section{Processed Channel: PDA $225.0 \mathrm{~nm}$}

\begin{tabular}{|l|l|r|r|r|r|}
\hline & Processed Channel & $\begin{array}{c}\text { Retention } \\
\text { Time }(\mathrm{min})\end{array}$ & Area & \% Area & Height \\
\hline 1 & PDA 225.0 nm & 4.518 & 286164 & 49.69 & 30563 \\
\hline 2 & PDA 225.0 nm & 4.956 & 289782 & 50.31 & 25915 \\
\hline
\end{tabular}

Enantioriched sample of $\left(S_{a}, R\right)-15$ :

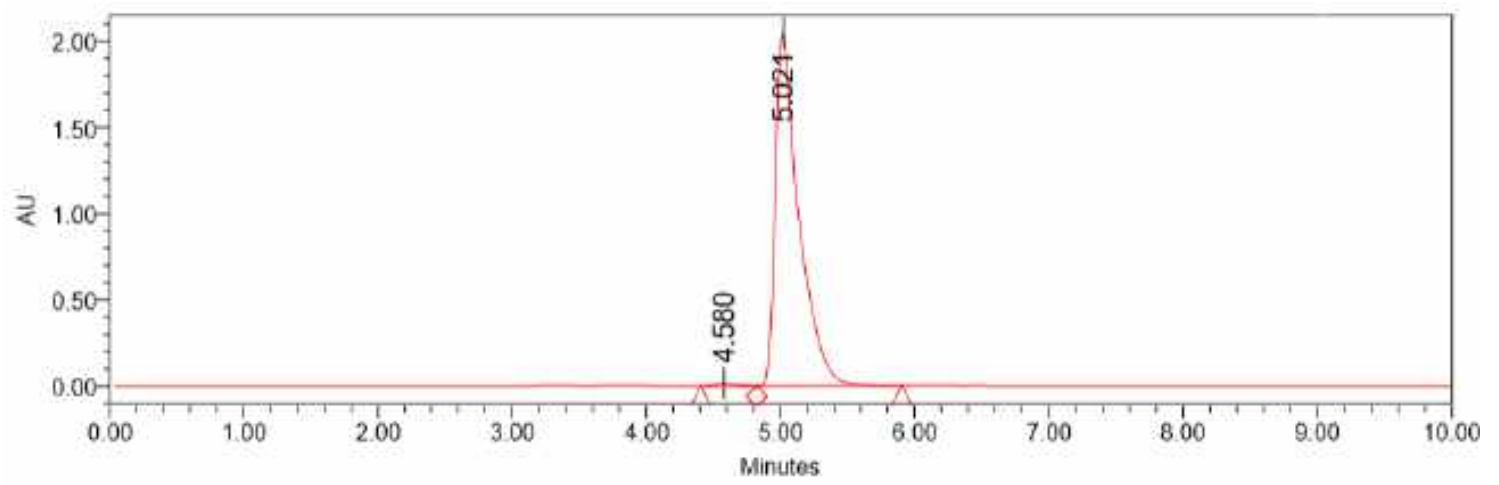

Processed Channel: PDA $225.0 \mathrm{~nm}$

\begin{tabular}{|c|c|c|c|c|c|}
\hline & Processed Channel & $\begin{array}{l}\text { Retention } \\
\text { Time (min) }\end{array}$ & Area & $\%$ Area & Height \\
\hline 1 & PDA $225.0 \mathrm{~nm}$ & 4.580 & 149742 & 0.60 & 15607 \\
\hline 2 & PDA $225.0 \mathrm{~nm}$ & 5.021 & 24743670 & 99.40 & 2046059 \\
\hline
\end{tabular}


${ }^{1} \mathrm{H}$ NMR $\left(400 \mathrm{MHz}, \mathrm{CDCl}_{3}\right)$ of $\left(S_{a,} S\right)-23$ :

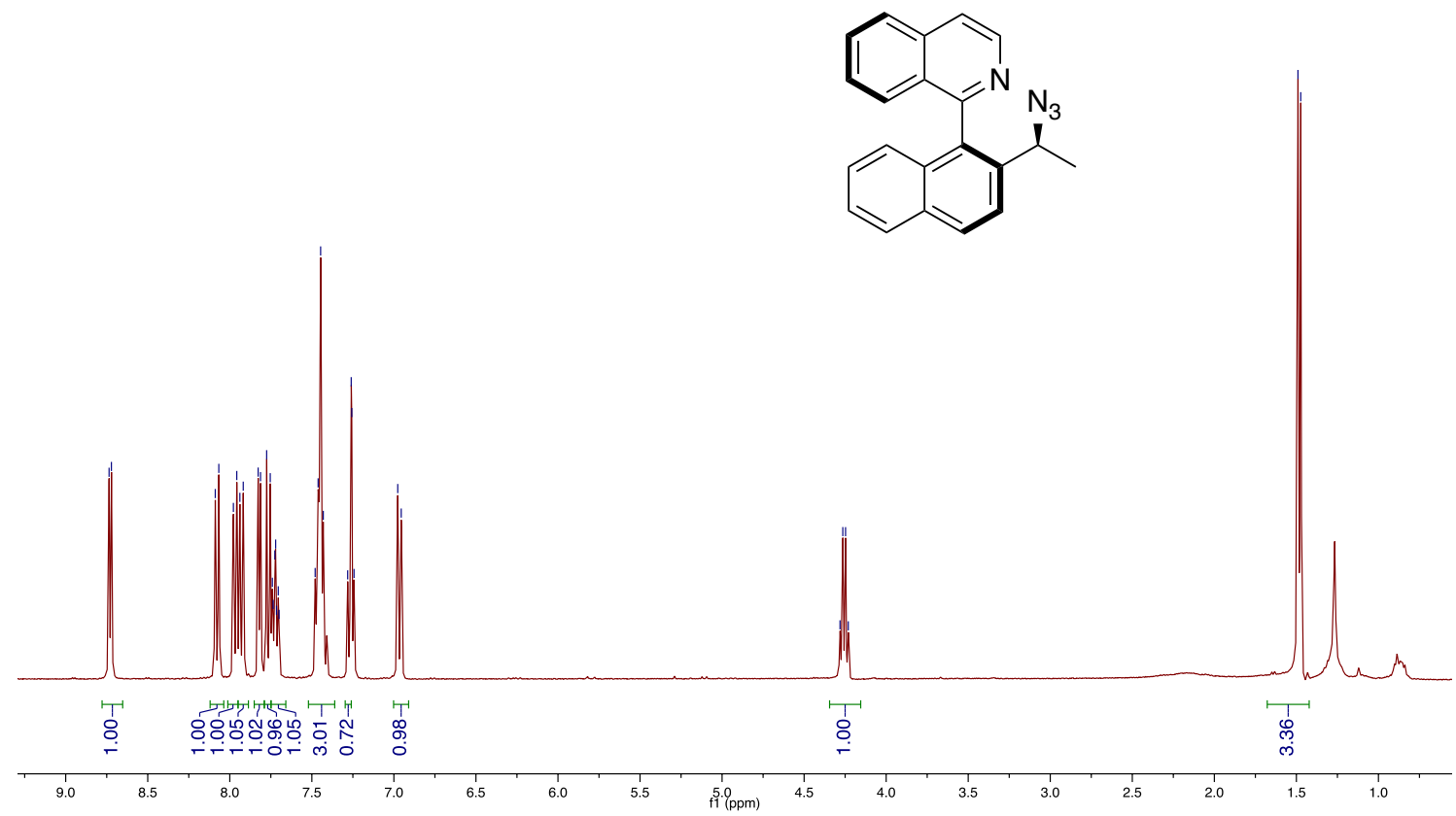

${ }^{13} \mathrm{C}$ NMR $\left(100 \mathrm{MHz}, \mathrm{CDCl}_{3}\right)$ of $\left(S_{a} S\right)-23$ :

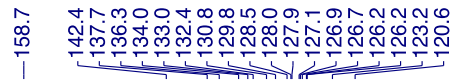

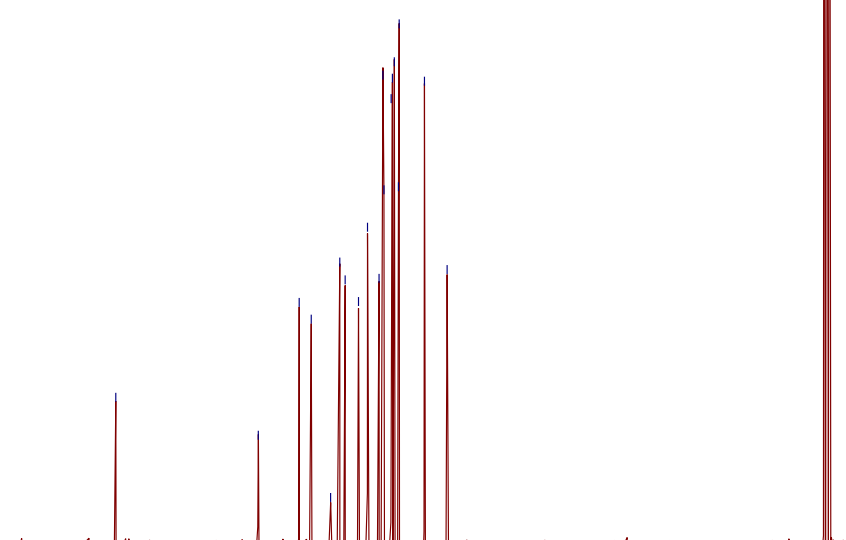<smiles>C[C@H](N)c1ccc2ccccc2c1-c1nccc2ccccc12</smiles> 
Racemic sample of $( \pm)$-23: IA column, $n$-hexane:isopropanol 95:5, F= $1 \mathrm{~mL} / \mathrm{min}, \mathrm{T}=$ $30^{\circ} \mathrm{C}$.

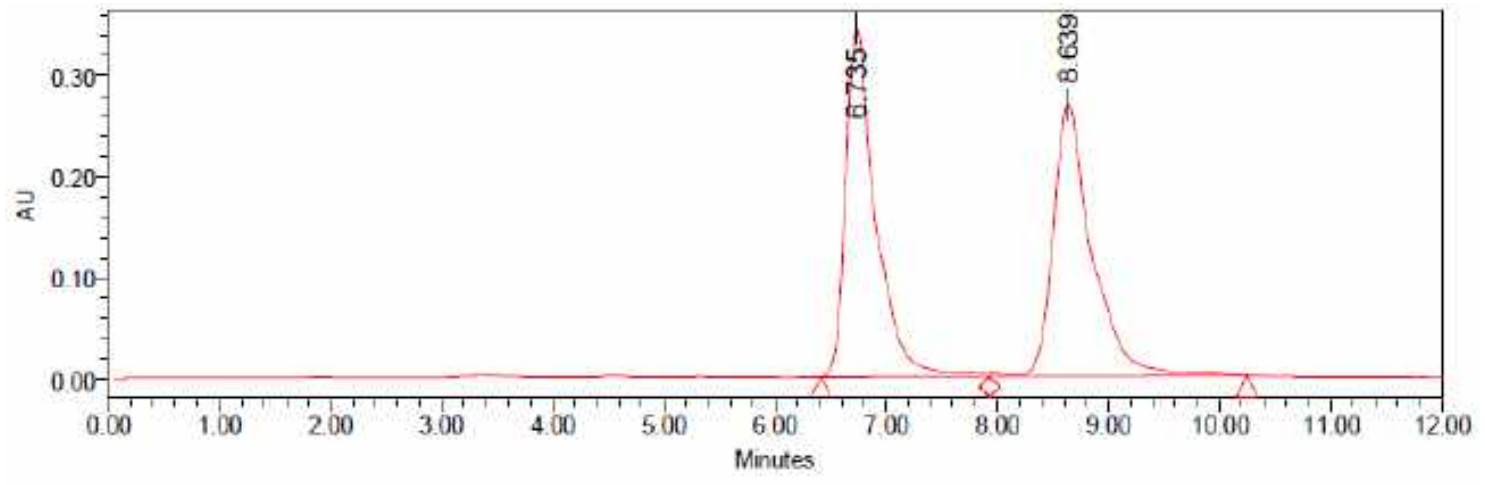

\section{Processed Channel: PDA $223.0 \mathrm{~nm}$}

\begin{tabular}{|l|l|r|c|r|r|}
\hline & Processed Channel & $\begin{array}{r}\text { Retention } \\
\text { Time }(\mathrm{min})\end{array}$ & Area & \% Area & Height \\
\hline 1 & PDA $223.0 \mathrm{~nm}$ & 6.735 & 6435762 & 49.82 & 344495 \\
\hline 2 & PDA $223.0 \mathrm{~nm}$ & 8.639 & 6482891 & 50.18 & 269891 \\
\hline
\end{tabular}

\section{Enantioriched sample of $\left(S_{a}, S\right)$-23:}

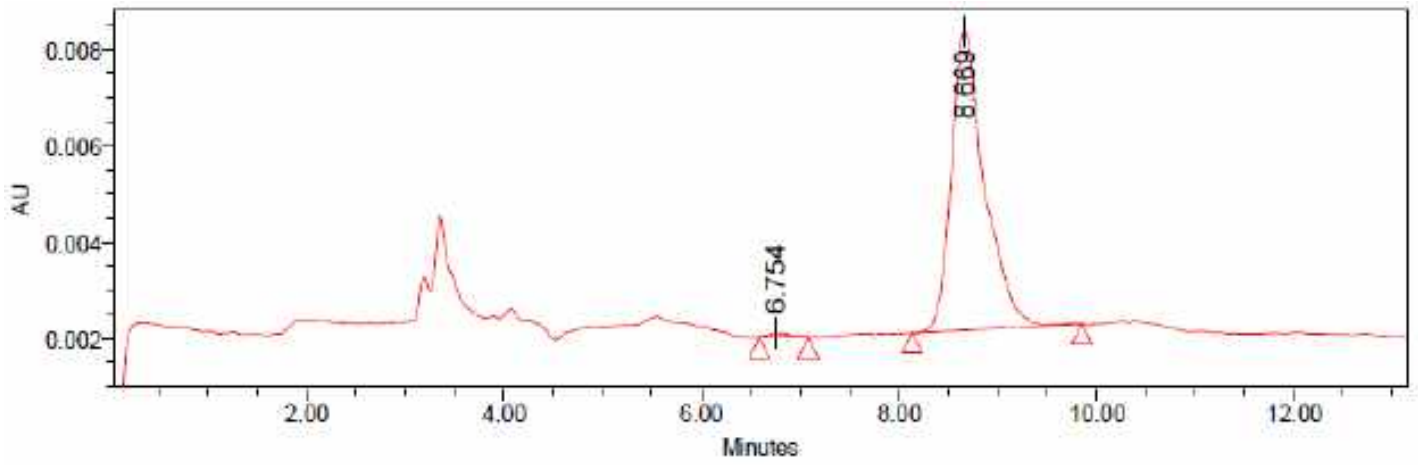

\section{Processed Channel: PDA $223.0 \mathrm{~nm}$}

\begin{tabular}{|r|l|r|r|r|r|}
\hline & Processed Channel & $\begin{array}{r}\text { Retention } \\
\text { Time }(\mathrm{min})\end{array}$ & Area & $\%$ Area & Height \\
\hline 1 & PDA 223.0 nm & 6.754 & 1479 & 1.01 & 103 \\
\hline 2 & PDA 223.0 nm & 8.669 & 144863 & 98.99 & 6197 \\
\hline
\end{tabular}


${ }^{1} \mathrm{H}$ NMR $\left(400 \mathrm{MHz}, \mathrm{CDCl}_{3}\right)$ of $\left(S_{a}, S\right)-\mathbf{2 4}$ :

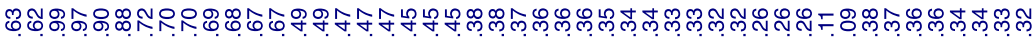

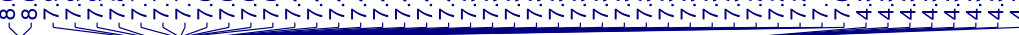

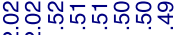

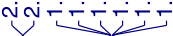<smiles>Cc1cccnc1-c1c(C(C)N)ccc2ccccc12</smiles>

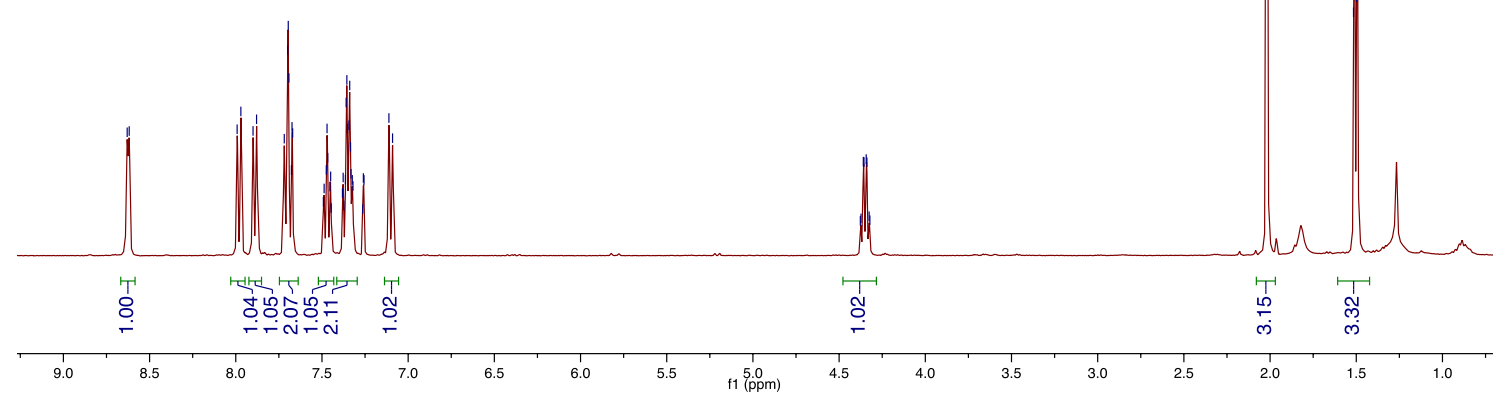

${ }^{13} \mathrm{C}$ NMR $\left(100 \mathrm{MHz}, \mathrm{CDCl}_{3}\right)$ of $\left(S_{a}, S\right)-24$ :

它
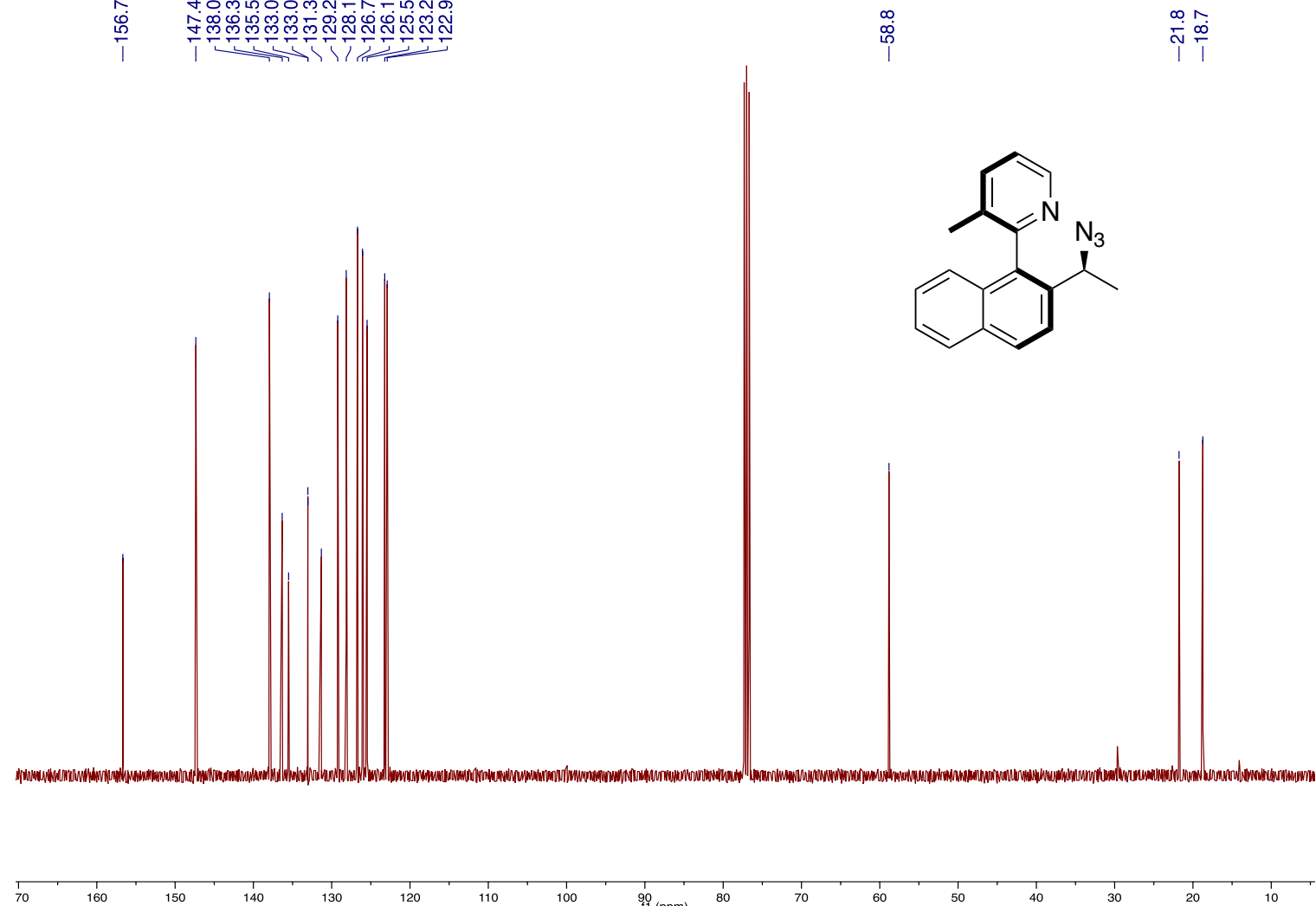

140

130 
Racemic sample of $( \pm)$-24: OD column, $n$-hexane:isopropanol 99:1, F= $1 \mathrm{~mL} / \mathrm{min}, \mathrm{T}=$ $30^{\circ} \mathrm{C}$.

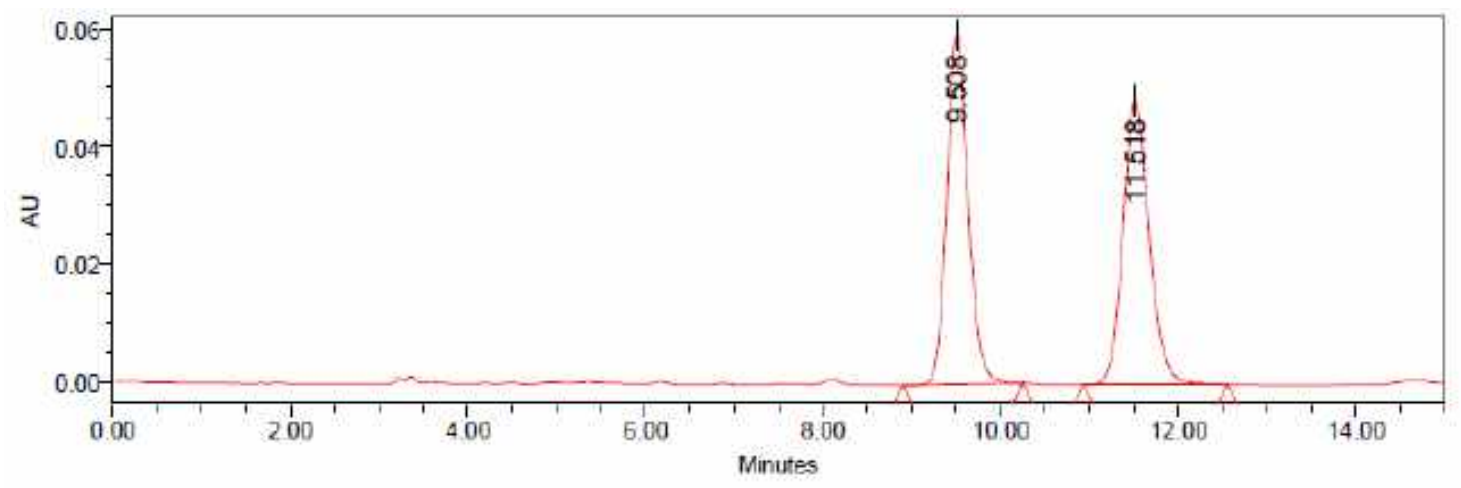

\section{Processed Channel: PDA $226.0 \mathrm{~nm}$}

\begin{tabular}{|l|l|r|r|r|r|}
\hline & Processed Channel & $\begin{array}{c}\text { Retention } \\
\text { Time (min) }\end{array}$ & Area & \% Area & Height \\
\hline 1 & PDA 226.0 nm & 9.508 & 989771 & 49.86 & 59435 \\
\hline 2 & PDA 226.0 nm & 11.518 & 995255 & 50.14 & 48305 \\
\hline
\end{tabular}

Enantioriched sample of $\left(S_{a}, S\right)-24$ :

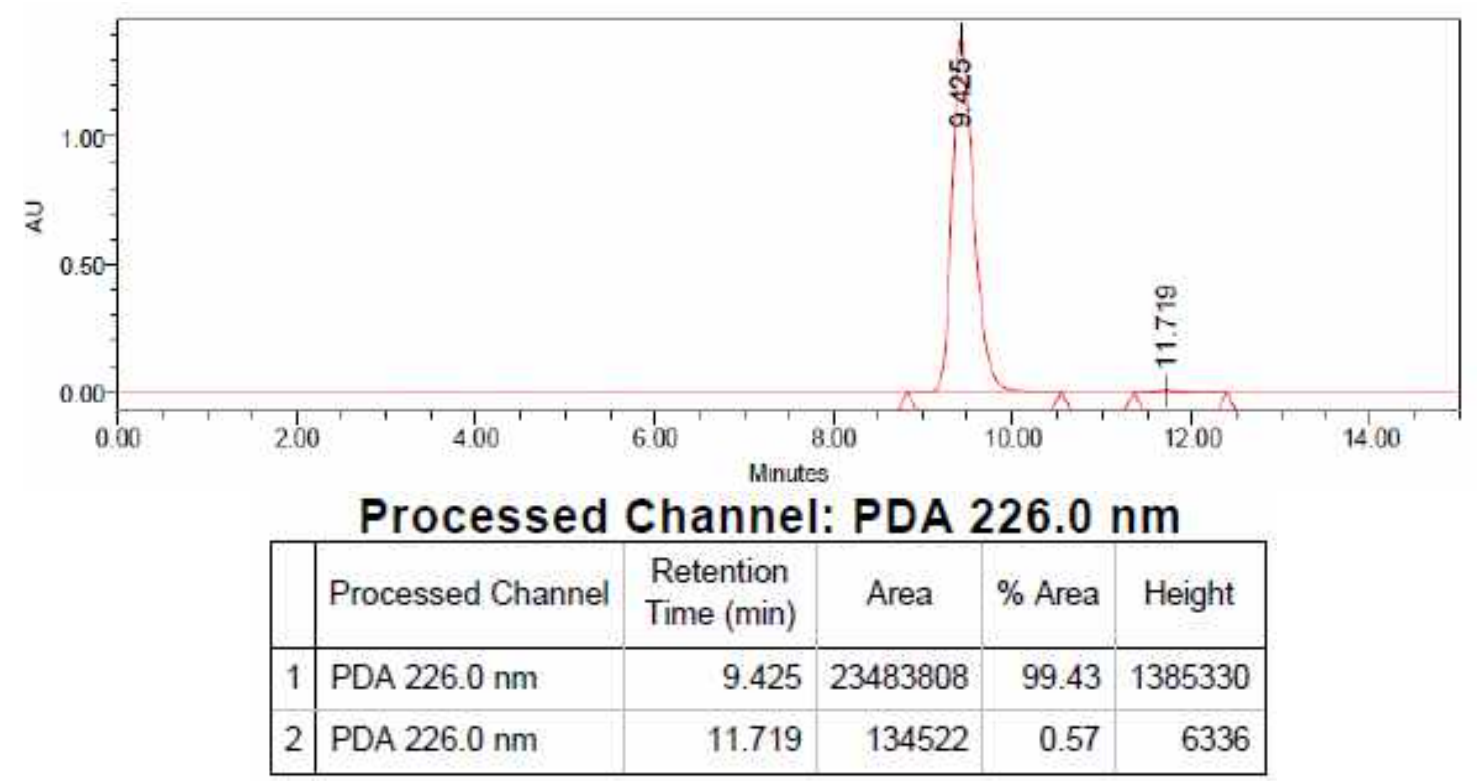


${ }^{1} \mathrm{H}$ NMR $\left(400 \mathrm{MHz}, \mathrm{CDCl}_{3}\right)$ of $\left(S_{a}, S\right)-\mathbf{2 5}$ :

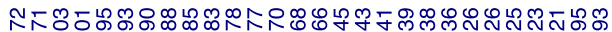

o

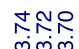<smiles>CC(N)c1ccc2ccccc2c1-c1nccc2ccccc12</smiles>
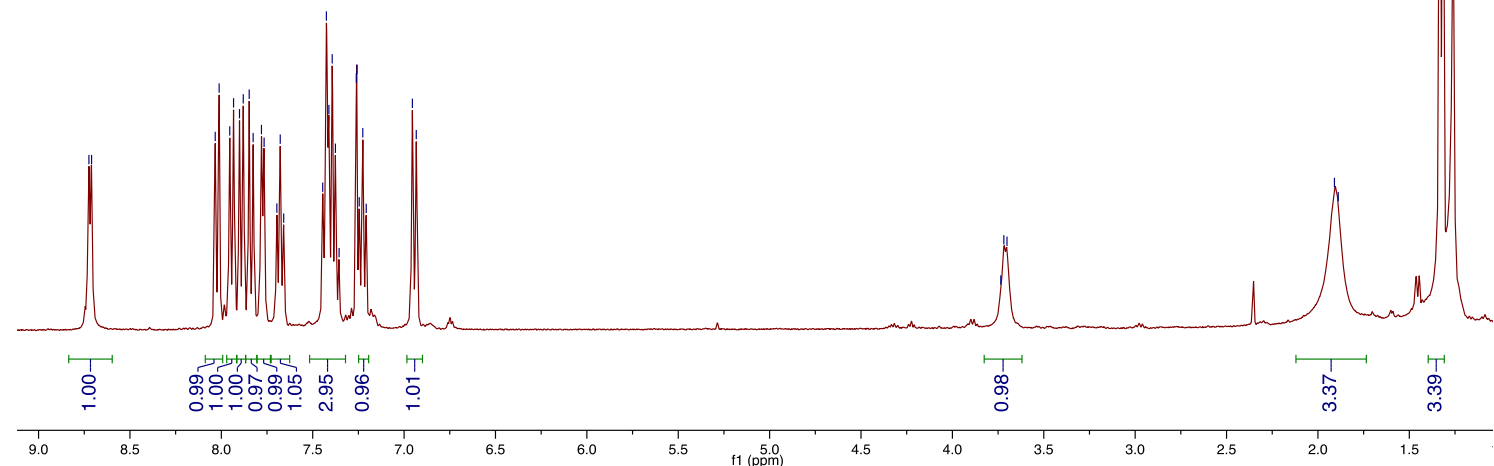

${ }^{13} \mathrm{C}$ NMR $\left(100 \mathrm{MHz}, \mathrm{CDCl}_{3}\right)$ of $\left(S_{a}, S\right)-25$ :
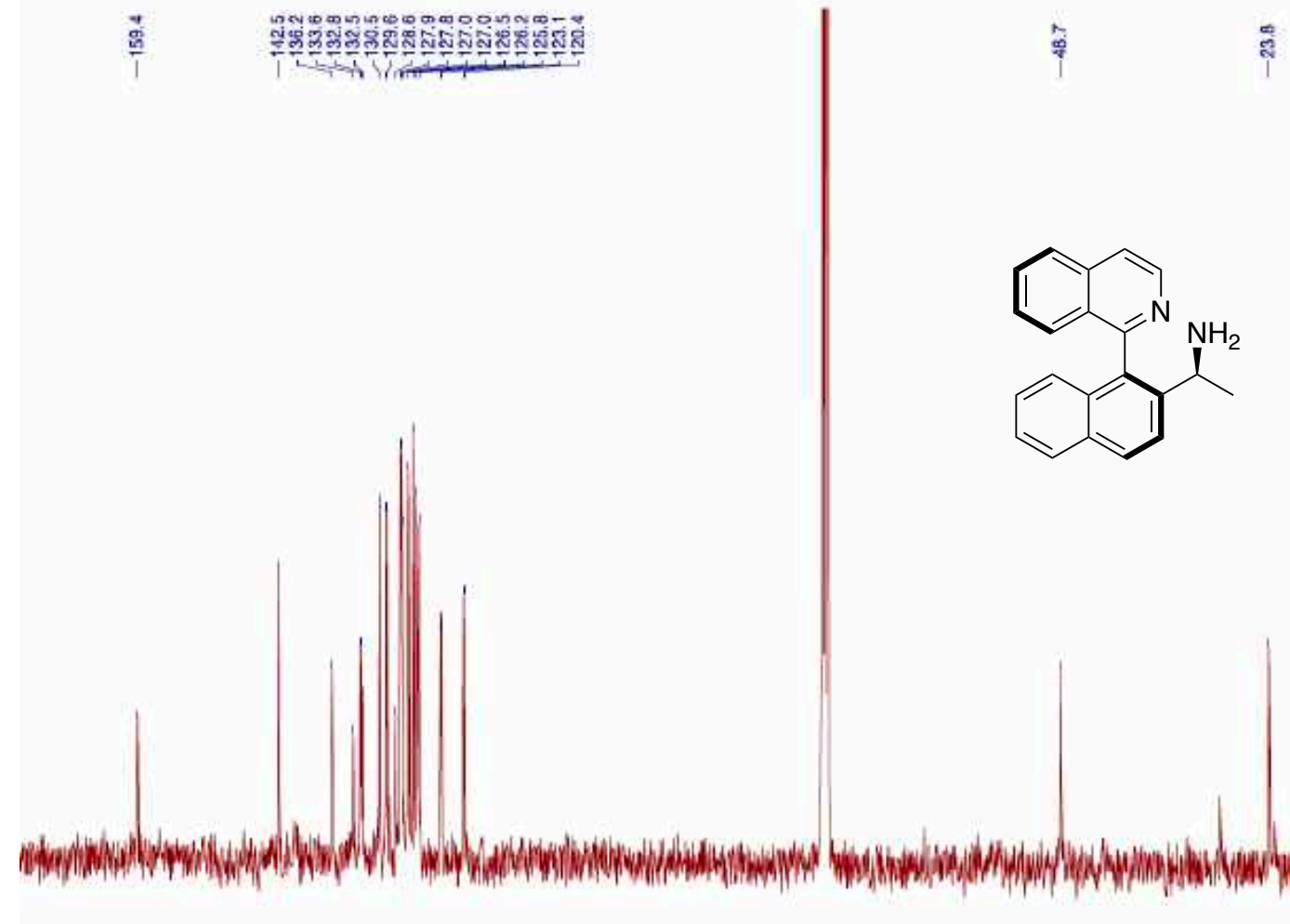

$160 \quad 150$

190

130 
Racemic sample of the benzoylated derivative of $( \pm)-25$ : IA column, $n$ hexane:isopropanol $85: 15, \mathrm{~F}=1 \mathrm{~mL} / \mathrm{min}, \mathrm{T}=30^{\circ} \mathrm{C}$.

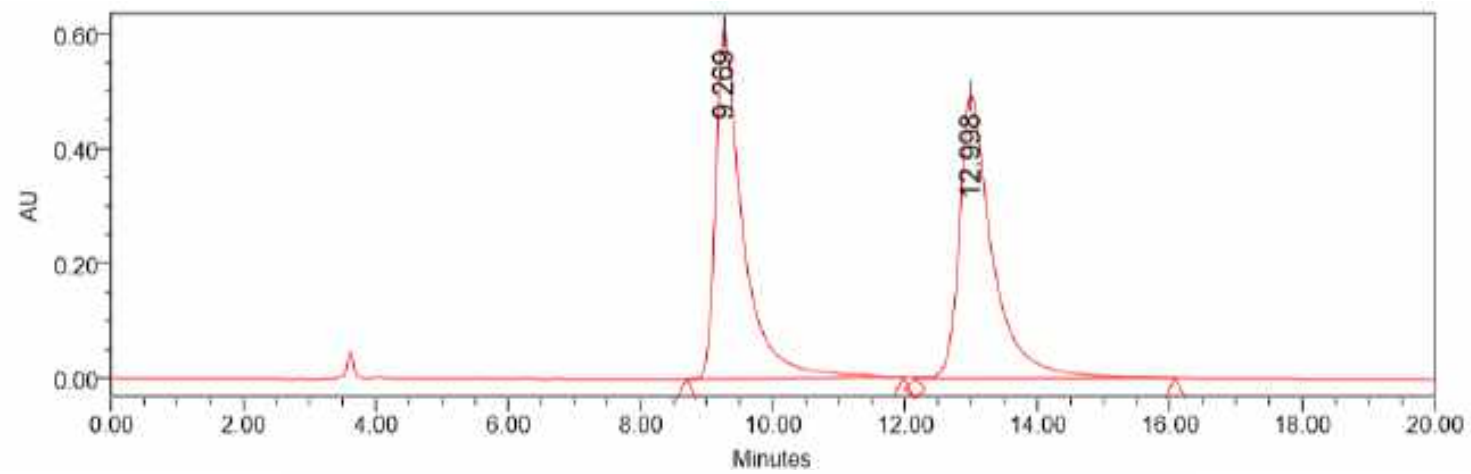

Processed Channel: PDA $223.0 \mathrm{~nm}$

\begin{tabular}{|l|l|r|r|r|r|}
\hline & Processed Channel & $\begin{array}{l}\text { Retention } \\
\text { Time (min) }\end{array}$ & Area & \% Area & Height \\
\hline 1 & PDA 223.0 nm & 9.269 & 17368190 & 50.29 & 606400 \\
\hline 2 & PDA 223.0 nm & 12.998 & 17165886 & 49.71 & 493436 \\
\hline
\end{tabular}

Enantioriched sample of the benzoylated derivative of $\left(S_{a}, S\right)-25$ :

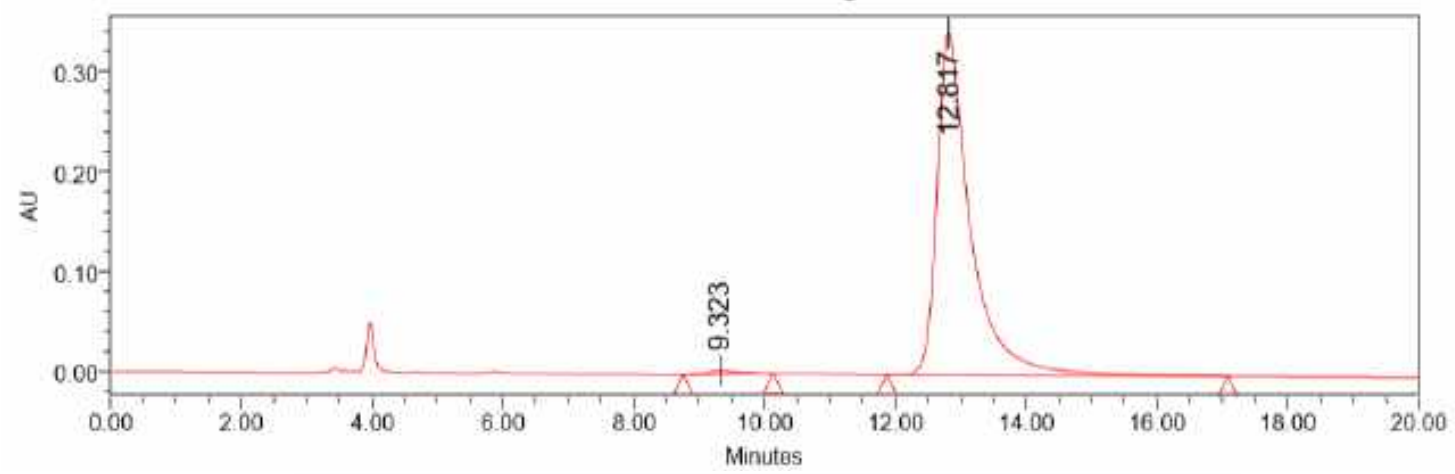

Processed Channel: PDA $223.0 \mathrm{~nm}$

\begin{tabular}{|r|l|r|r|r|r|}
\hline & Processed Channel & $\begin{array}{r}\text { Retention } \\
\text { Time }(\mathrm{min})\end{array}$ & Area & \% Area & Height \\
\hline 1 & PDA 223.0 nm & 9.323 & 108528 & 0.88 & 3588 \\
\hline 2 & PDA 223.0 nm & 12.817 & 12213691 & 99.12 & 342111 \\
\hline
\end{tabular}


${ }^{1} \mathrm{H}$ NMR $\left(400 \mathrm{MHz}, \mathrm{CDCl}_{3}\right)$ of $\left(S_{a}, S\right)-\mathbf{2 6}$ :

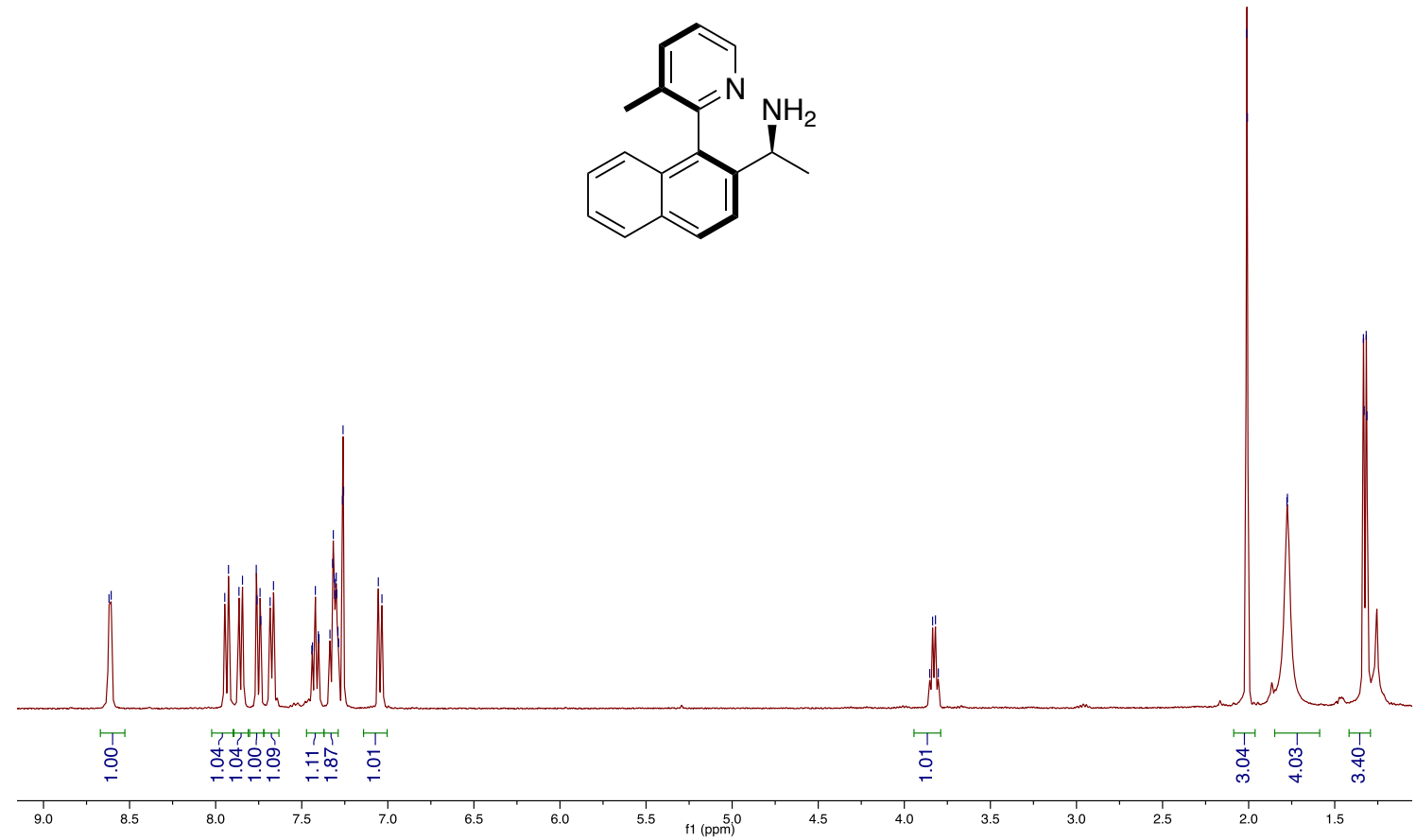

${ }^{13} \mathrm{C}$ NMR $\left(100 \mathrm{MHz}, \mathrm{CDCl}_{3}\right)$ of $\left(S_{a}, S\right)-\mathbf{2 6}$ :

l.
r.

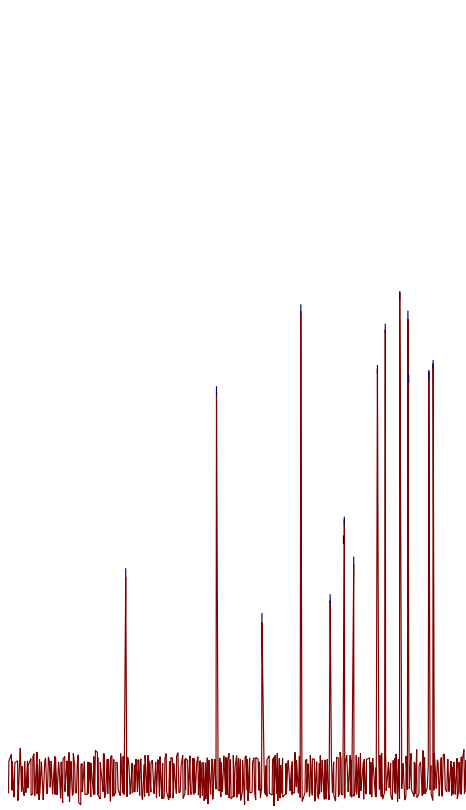

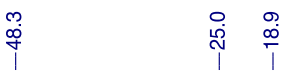

(N)
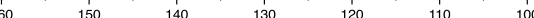

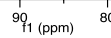


Racemic sample of the benzoylated derivative of $( \pm)$-26: IA column, $n$ hexane:isopropanol $85: 15, \mathrm{~F}=1 \mathrm{~mL} / \mathrm{min}, \mathrm{T}=30^{\circ} \mathrm{C}$.

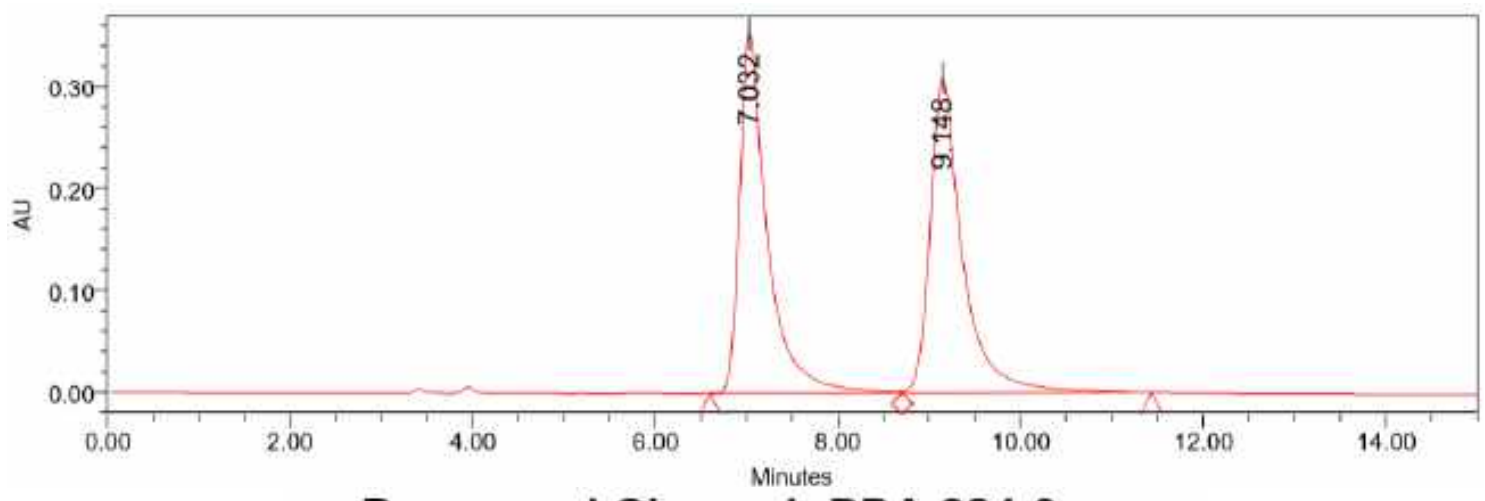

Processed Channel: PDA $224.0 \mathrm{~nm}$

\begin{tabular}{|l|l|r|c|r|r|}
\hline & Processed Channel & $\begin{array}{r}\text { Retention } \\
\text { Time }(m i n)\end{array}$ & Area & \% Area & Height \\
\hline 1 & PDA 224.0 nm & 7.032 & 7500797 & 49.57 & 353019 \\
\hline 2 & PDA 224.0 nm & 9.148 & 7631604 & 50.43 & 309313 \\
\hline
\end{tabular}

Enantioriched sample of the benzoylated derivative of $\left(S_{a} S\right)-26$ :

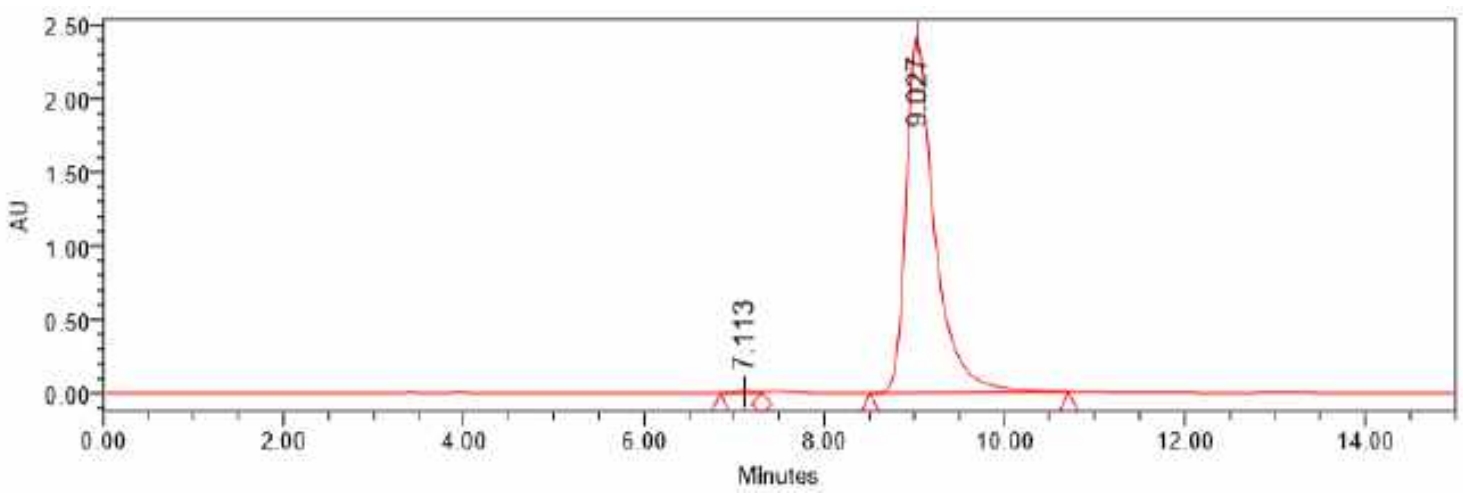

Processed Channel: PDA $224.0 \mathrm{~nm}$

\begin{tabular}{|l|l|r|r|r|r|}
\hline & Processed Channel & $\begin{array}{r}\text { Retention } \\
\text { Time }(\mathrm{min})\end{array}$ & \multicolumn{1}{|c|}{ Area } & \% Area & Height \\
\hline 1 & PDA 224.0 nm & 7.113 & 201265 & 0.38 & 11251 \\
\hline 2 & PDA 224.0 nm & 9.027 & 53345104 & 99.62 & 2416732 \\
\hline
\end{tabular}


${ }^{1} \mathrm{H} \mathrm{NMR}\left(400 \mathrm{MHz}, \mathrm{CDCl}_{3}\right)$ of $\left(R_{a}, R, R, S\right)-7 \mathbf{h}:$

i

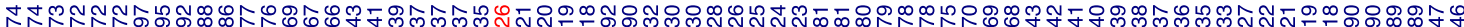

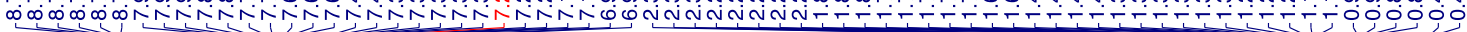

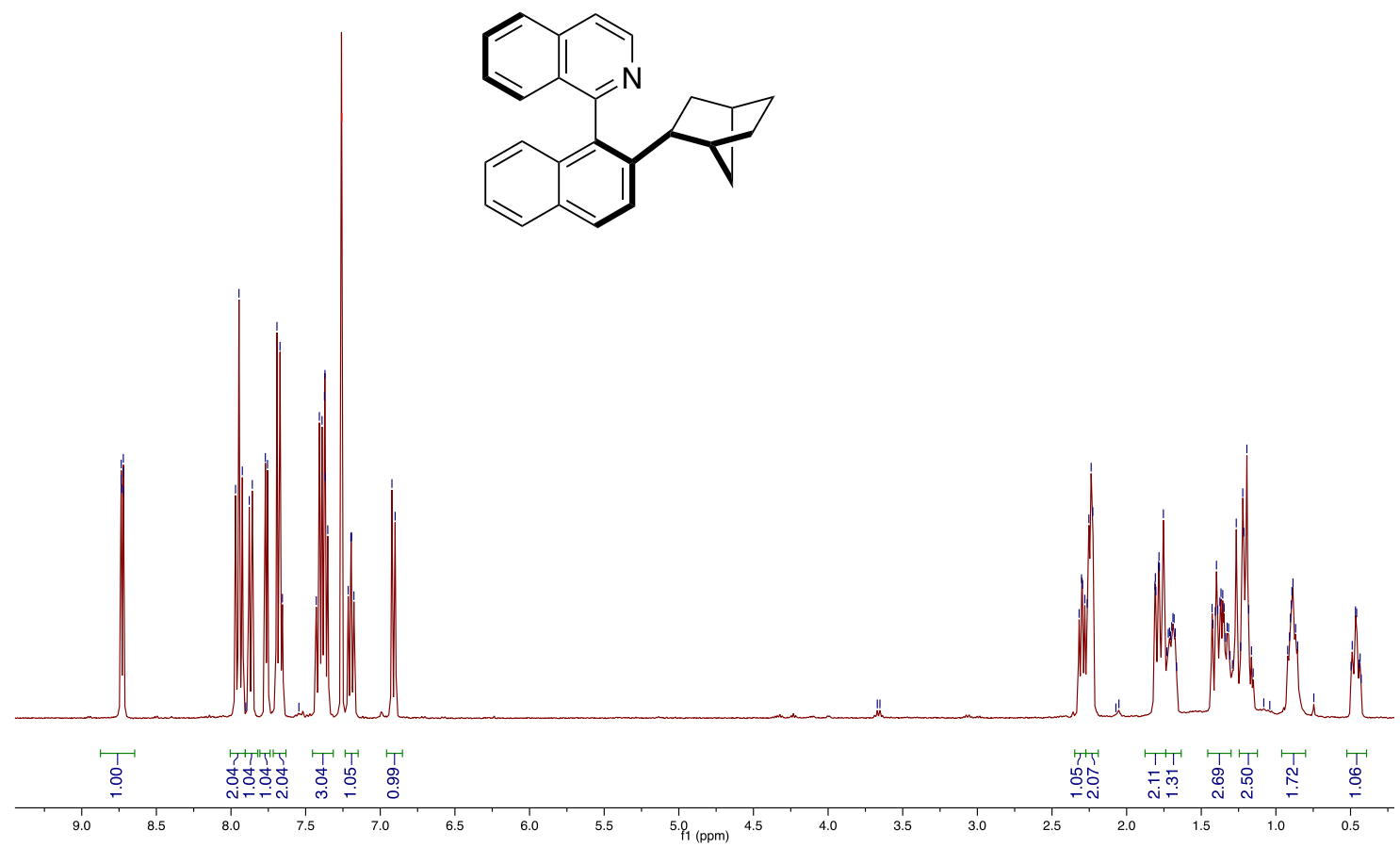

${ }^{13} \mathrm{C} \mathrm{NMR}\left(100 \mathrm{MHz}, \mathrm{CDCl}_{3}\right)$ of $\left(R_{a}, R, R, S\right)-\mathbf{7 h}$ :

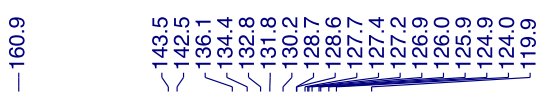

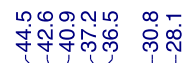<smiles>c1ccc2c(-c3cccc4c(C5CC6CCC5C6)cccc34)cccc2c1</smiles>

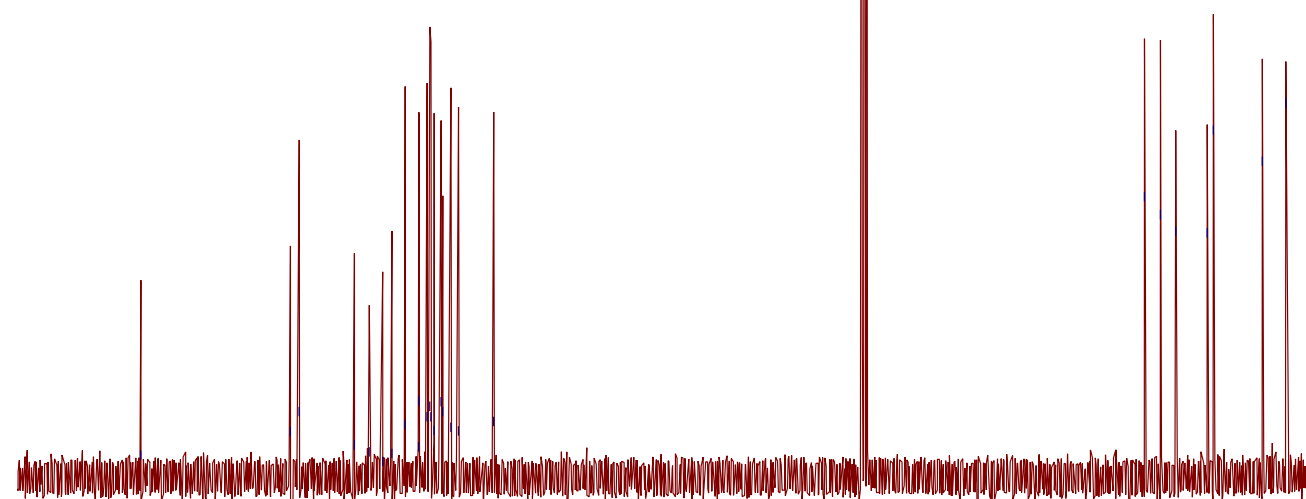

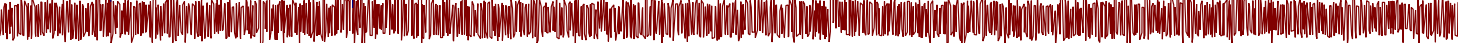

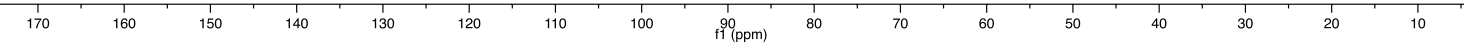


Racemic sample of $( \pm)-7 \mathbf{h}$ : OD column, $n$-hexane:isopropanol 99:1, F= $1 \mathrm{~mL} / \mathrm{min}, \mathrm{T}=$ $30^{\circ} \mathrm{C}$.

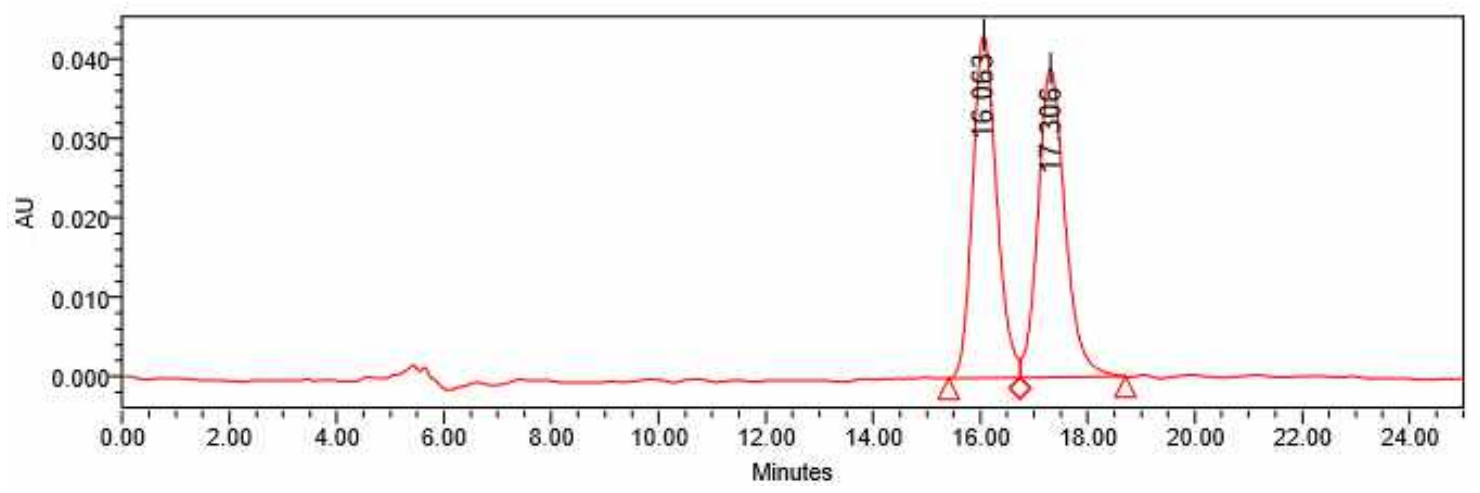

Processed Channel: PDA $227.0 \mathrm{~nm}$

\begin{tabular}{|l|l|r|r|r|r|}
\hline & Processed Channel & $\begin{array}{c}\text { Retention } \\
\text { Time (min) }\end{array}$ & Area & \% Area & Height \\
\hline 1 & PDA 227.0 nm & 16.063 & 1342374 & 49.43 & 43378 \\
\hline 2 & PDA 227.0 nm & 17.306 & 1373366 & 50.57 & 36966 \\
\hline
\end{tabular}

Enantioriched sample of $\left(R_{a}, R, R, S\right)-7 \mathbf{h}$ :

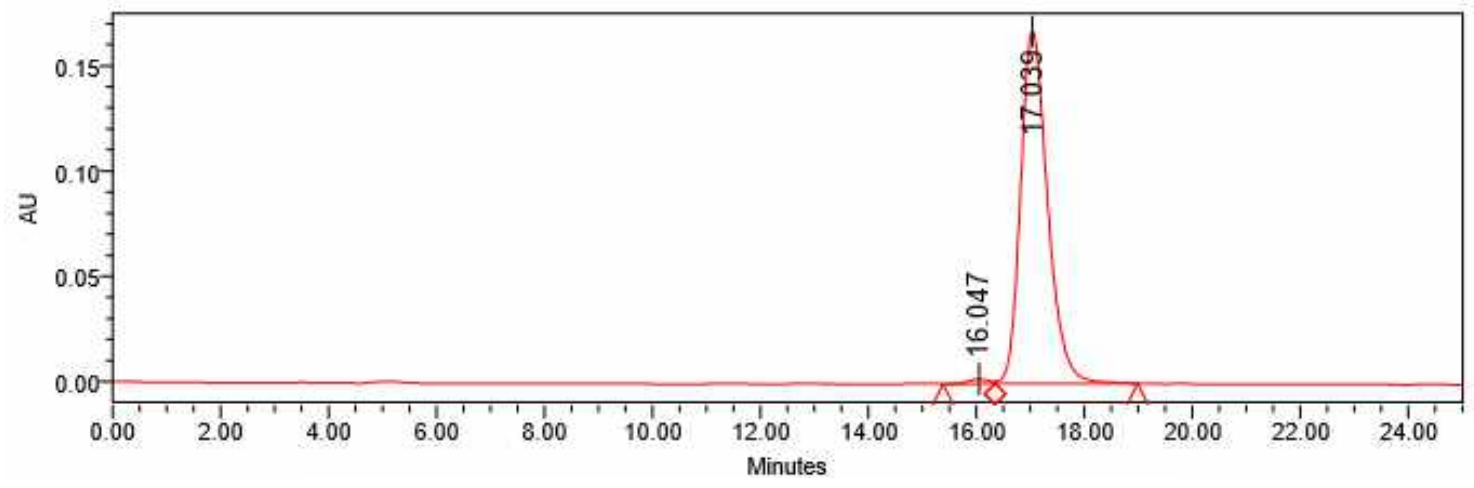

Processed Channel: PDA $227.0 \mathrm{~nm}$

\begin{tabular}{|r|l|r|r|r|r|}
\hline & Processed Channel & $\begin{array}{r}\text { Retention } \\
\text { Time (min) }\end{array}$ & Area & \% Area & Height \\
\hline 1 & PDA 227.0 nm & 16.047 & 61632 & 1.05 & 2197 \\
\hline 2 & PDA $227.0 \mathrm{~nm}$ & 17.039 & 5818771 & 98.95 & 167351 \\
\hline
\end{tabular}


${ }^{1} \mathrm{H}$ NMR $\left(400 \mathrm{MHz}, \mathrm{CDCl}_{3}\right)$ of $\left(R_{a}, R, R, S, R, S\right)-7 \mathbf{i}$ :

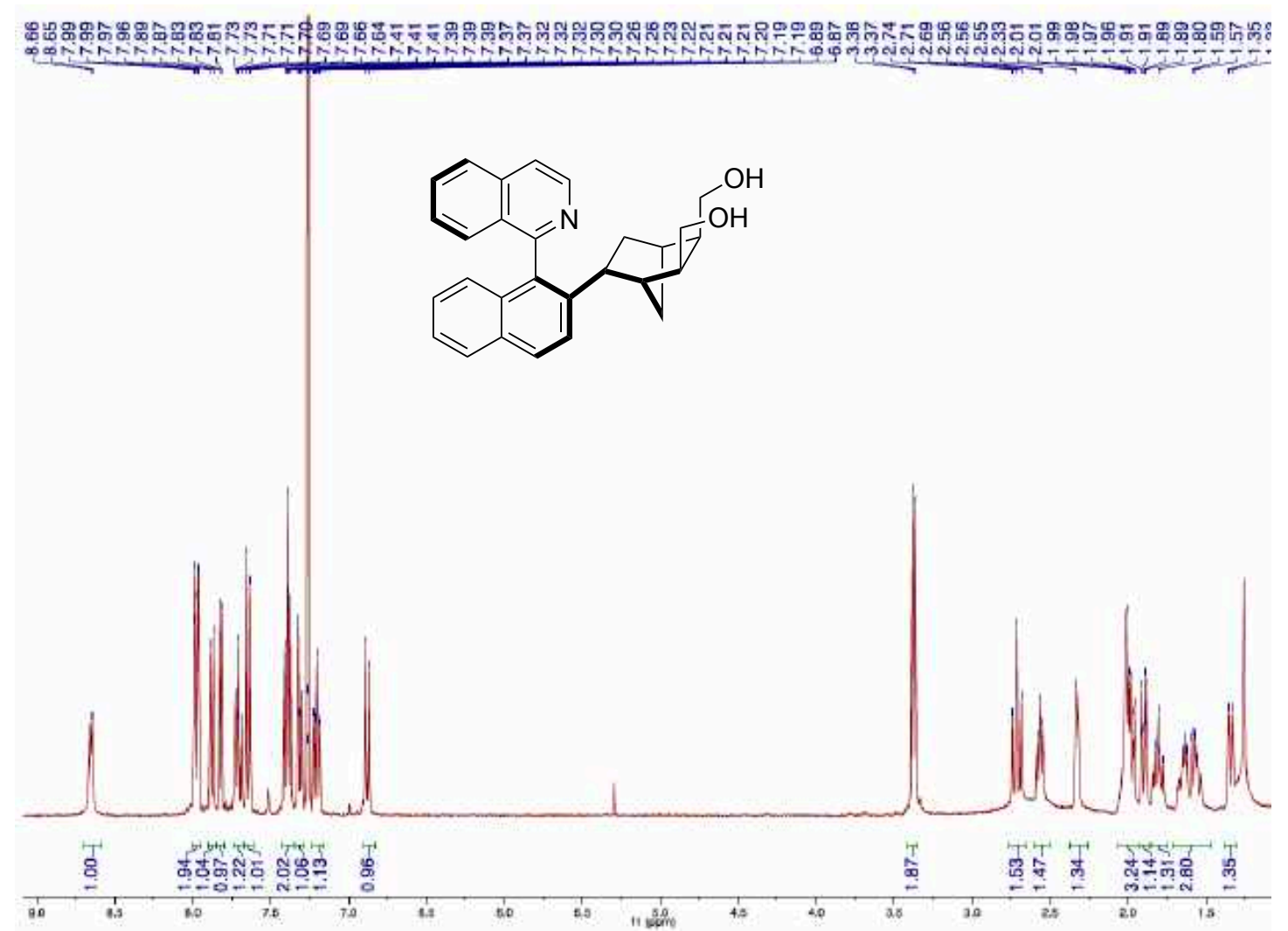

${ }^{13} \mathrm{C}$ NMR $\left(100 \mathrm{MHz}, \mathrm{CDCl}_{3}\right)$ of $\left(R_{a} R, R, S, R, S\right)-7 \mathbf{i}:$

$\frac{10}{1}$
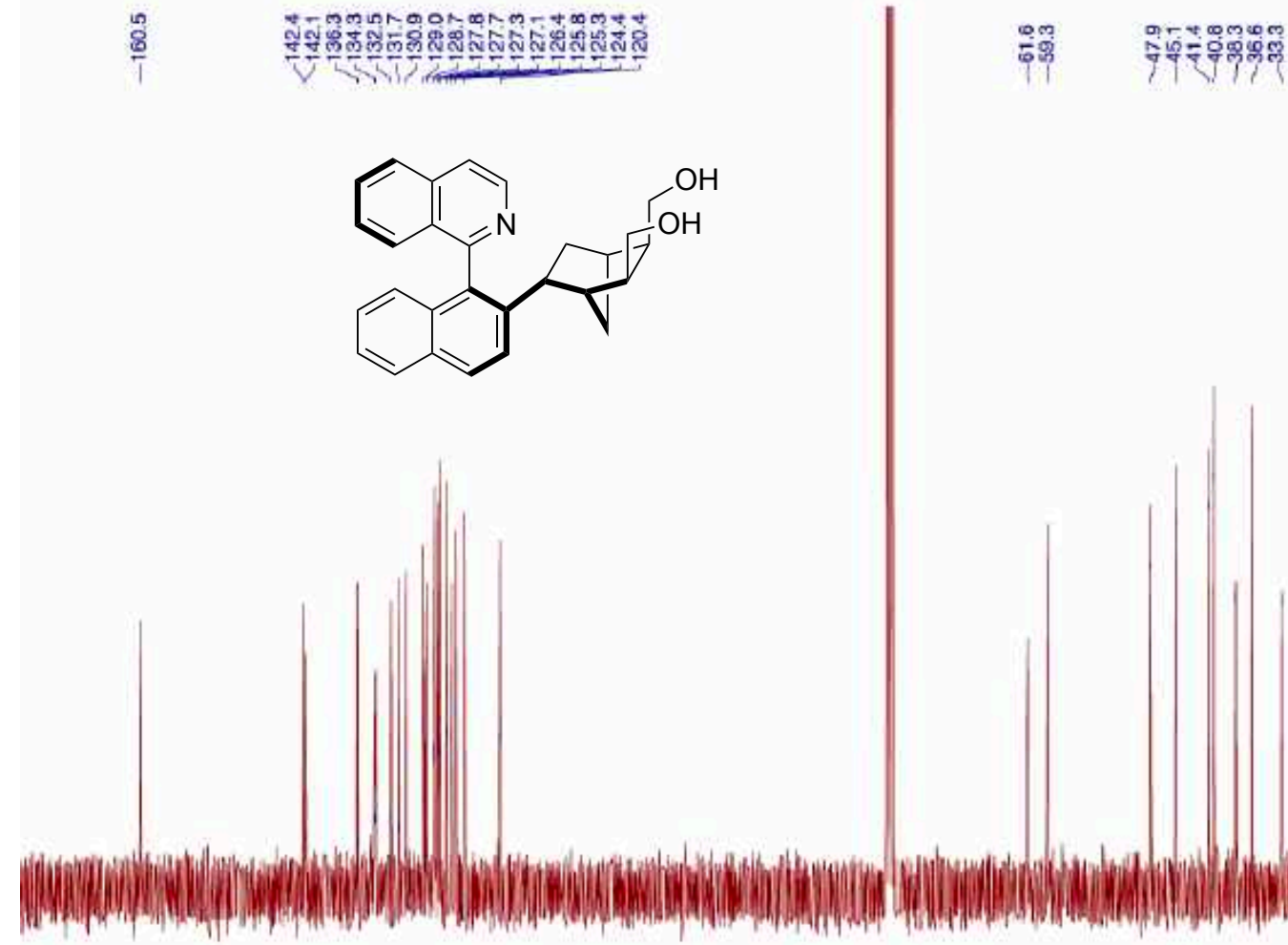
Racemic sample of $( \pm)$-7i: AS-H column, $n$-hexane:isopropanol $80: 20, F=1 \mathrm{~mL} / \mathrm{min}$, $\mathrm{T}=30^{\circ} \mathrm{C}$.

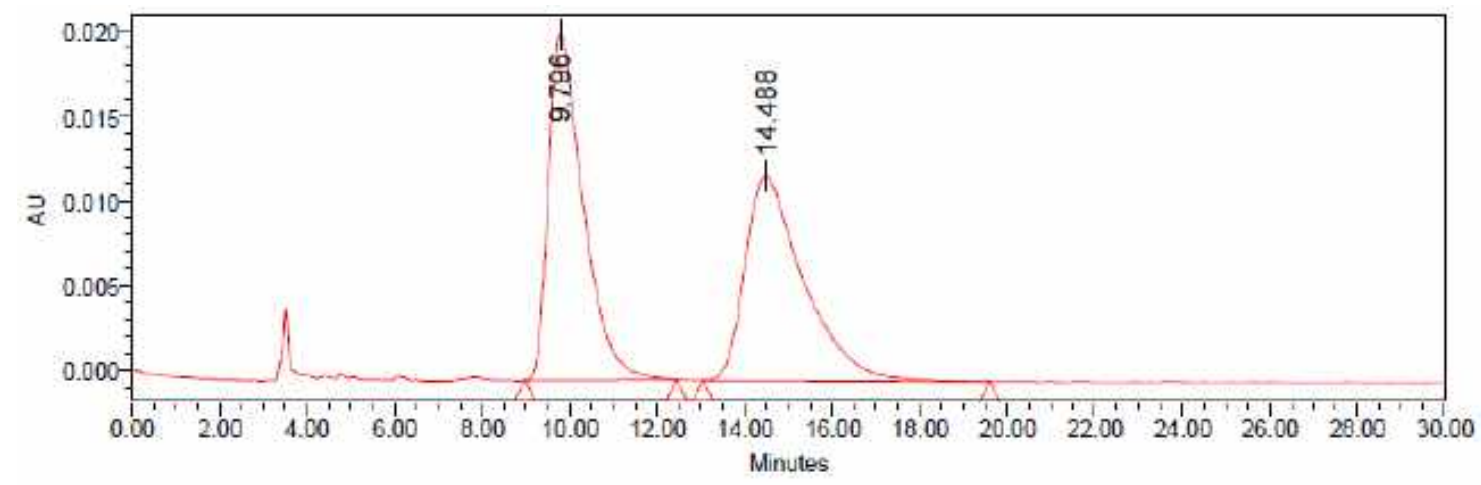

\section{Processed Channel: PDA $227.0 \mathrm{~nm}$}

\begin{tabular}{|l|l|r|c|c|c|}
\hline & Processed Channel & $\begin{array}{c}\text { Retention } \\
\text { Time }(\mathrm{min})\end{array}$ & Area & \% Area & Height \\
\hline 1 & PDA $227.0 \mathrm{~nm}$ & 9.796 & 1132233 & 50.29 & 20430 \\
\hline 2 & PDA $227.0 \mathrm{~nm}$ & 14.488 & 1119398 & 49.71 & 12091 \\
\hline
\end{tabular}

Enantioriched sample of $\left(R_{a}, R, R, S, R, S\right)-7 \mathrm{i}$ :

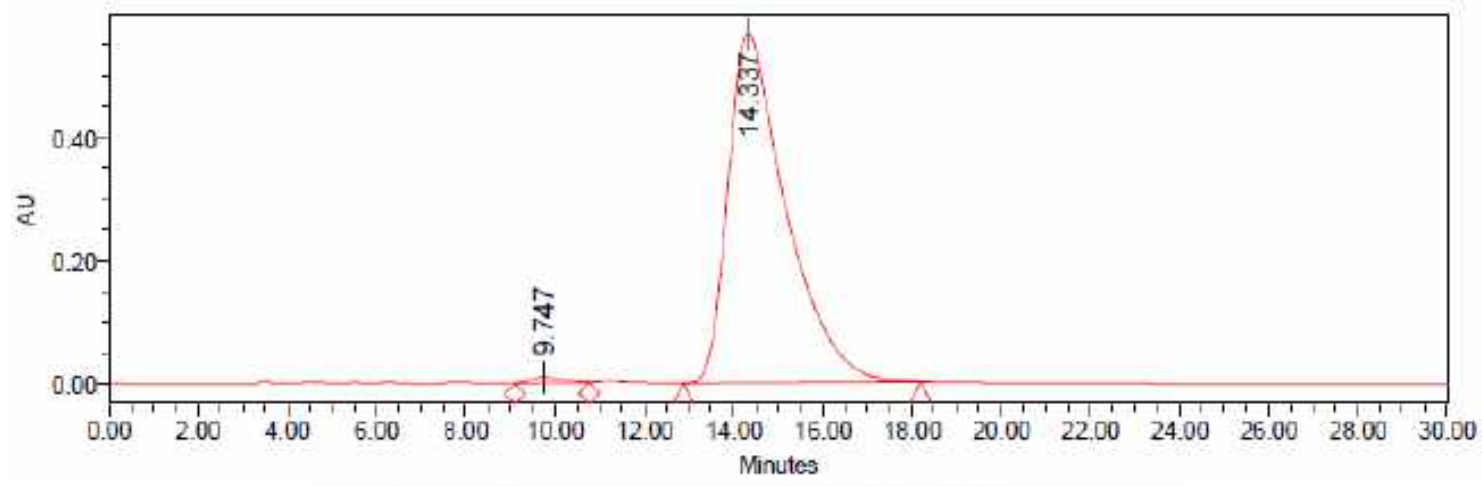

Processed Channel: PDA $227.0 \mathrm{~nm}$

\begin{tabular}{|l|l|r|r|r|r|}
\hline & Processed Channel & $\begin{array}{r}\text { Retention } \\
\text { Time }(\mathrm{min})\end{array}$ & Area & \% Area & Height \\
\hline 1 & PDA $227.0 \mathrm{~nm}$ & 9.747 & 509409 & 0.98 & 10444 \\
\hline 2 & PDA $227.0 \mathrm{~nm}$ & 14.337 & 51270376 & 99.02 & 568618 \\
\hline
\end{tabular}


${ }^{1} \mathrm{H}$ NMR $\left(400 \mathrm{MHz}, \mathrm{CDCl}_{3}\right)$ of $\left(R_{a}, R, R, S\right)-\mathbf{8 h}$ :

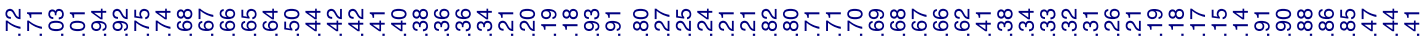
o

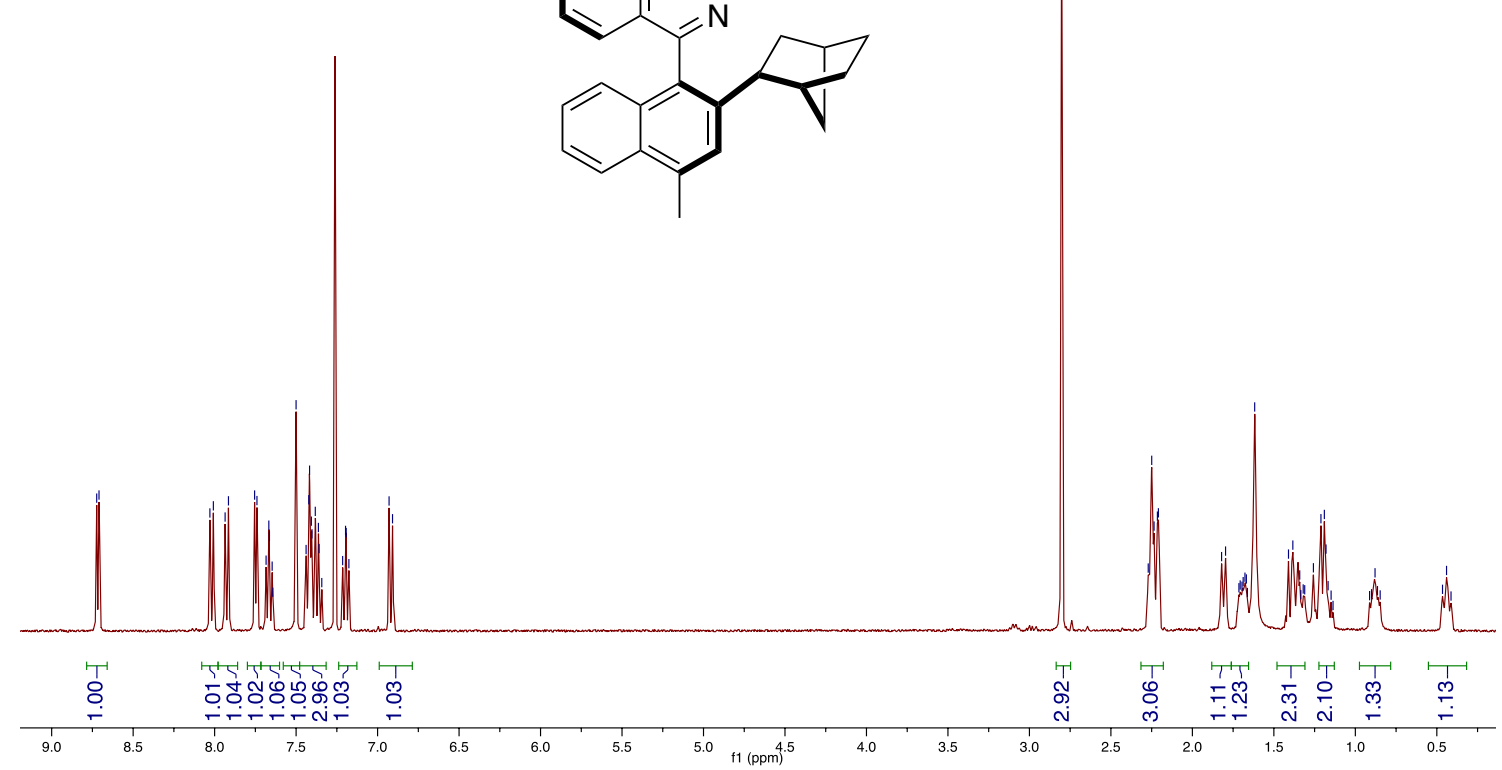

${ }^{13} \mathrm{C} \mathrm{NMR}\left(100 \mathrm{MHz}, \mathrm{CDCl}_{3}\right)$ of $\left(R_{a}, R, R, S\right)-\mathbf{8 h}$ :

产<smiles>Cc1cc(C2CC3CCC2C3)c(-c2nccc3ccccc23)c2ccccc12</smiles>

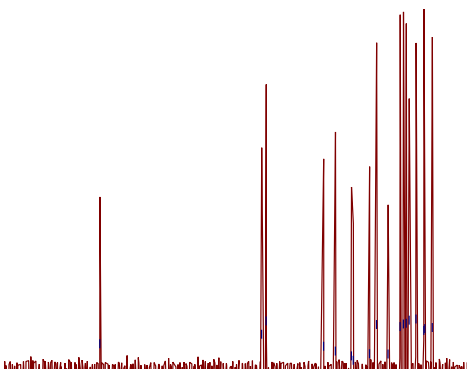

IF

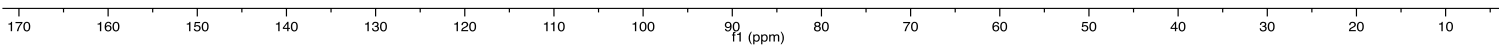


Racemic sample of $( \pm)-8 h$ : OD column, $n$-hexane:isopropanol 95:5, F= $1 \mathrm{~mL} / \mathrm{min}, \mathrm{T}=$ $30^{\circ} \mathrm{C}$.

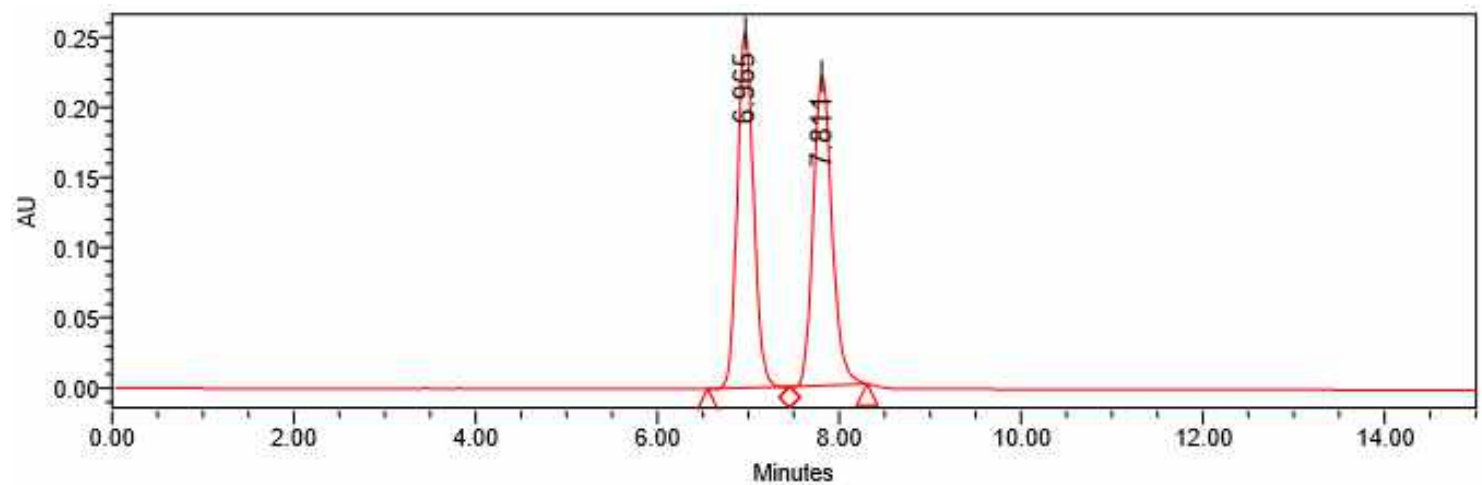

Processed Channel: PDA 231.0 nm

\begin{tabular}{|r|l|r|r|r|r|}
\hline & Processed Channel & $\begin{array}{r}\text { Retention } \\
\text { Time (min) }\end{array}$ & Area & \% Area & Height \\
\hline 1 & PDA $231.0 \mathrm{~nm}$ & 6.965 & 3143566 & 50.02 & 254072 \\
\hline 2 & PDA 231.0 nm & 7.811 & 3140792 & 49.98 & 220781 \\
\hline
\end{tabular}

\section{Enantioriched sample of $\left(R_{a}, R, R, S\right)-8 \mathbf{h}$ :}

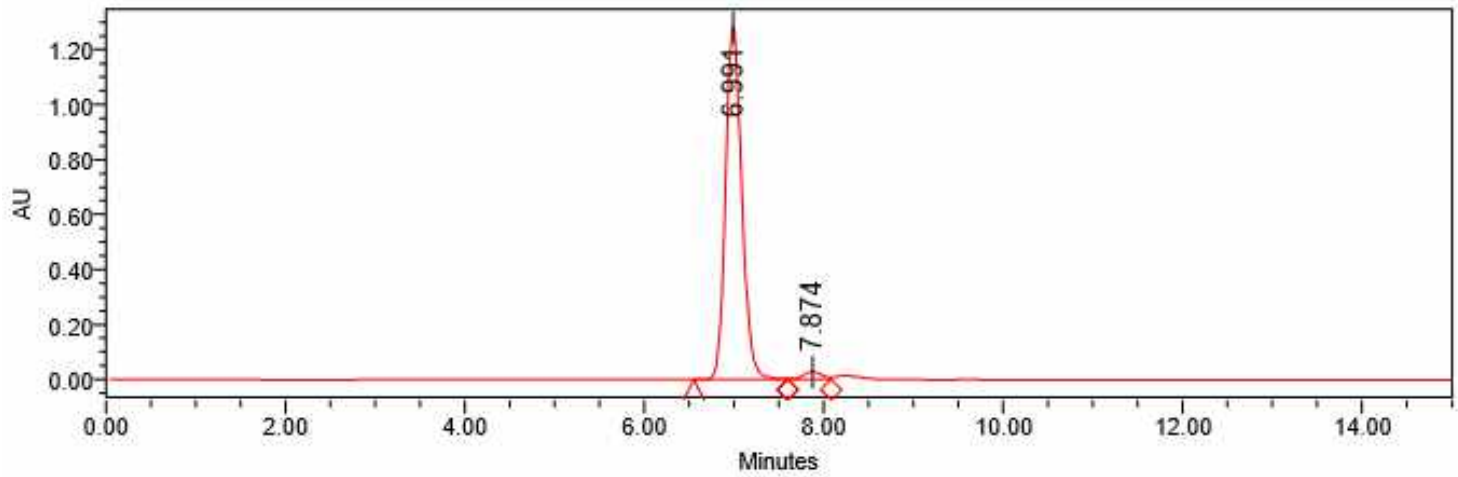

Processed Channel: PDA $231.0 \mathrm{~nm}$

\begin{tabular}{|r|l|r|r|r|r|}
\hline & Processed Channel & $\begin{array}{c}\text { Retention } \\
\text { Time (min) }\end{array}$ & Area & \% Area & Height \\
\hline 1 & PDA 231.0 nm & 6.991 & 16209233 & 97.44 & 1287853 \\
\hline 2 & PDA $231.0 \mathrm{~nm}$ & 7.874 & 425440 & 2.56 & 28563 \\
\hline
\end{tabular}


${ }^{1} \mathrm{H}$ NMR $\left(400 \mathrm{MHz}, \mathrm{CDCl}_{3}\right)$ of $\left(R_{a}, R, R, S, R, S\right)-\mathbf{8 i}$ :
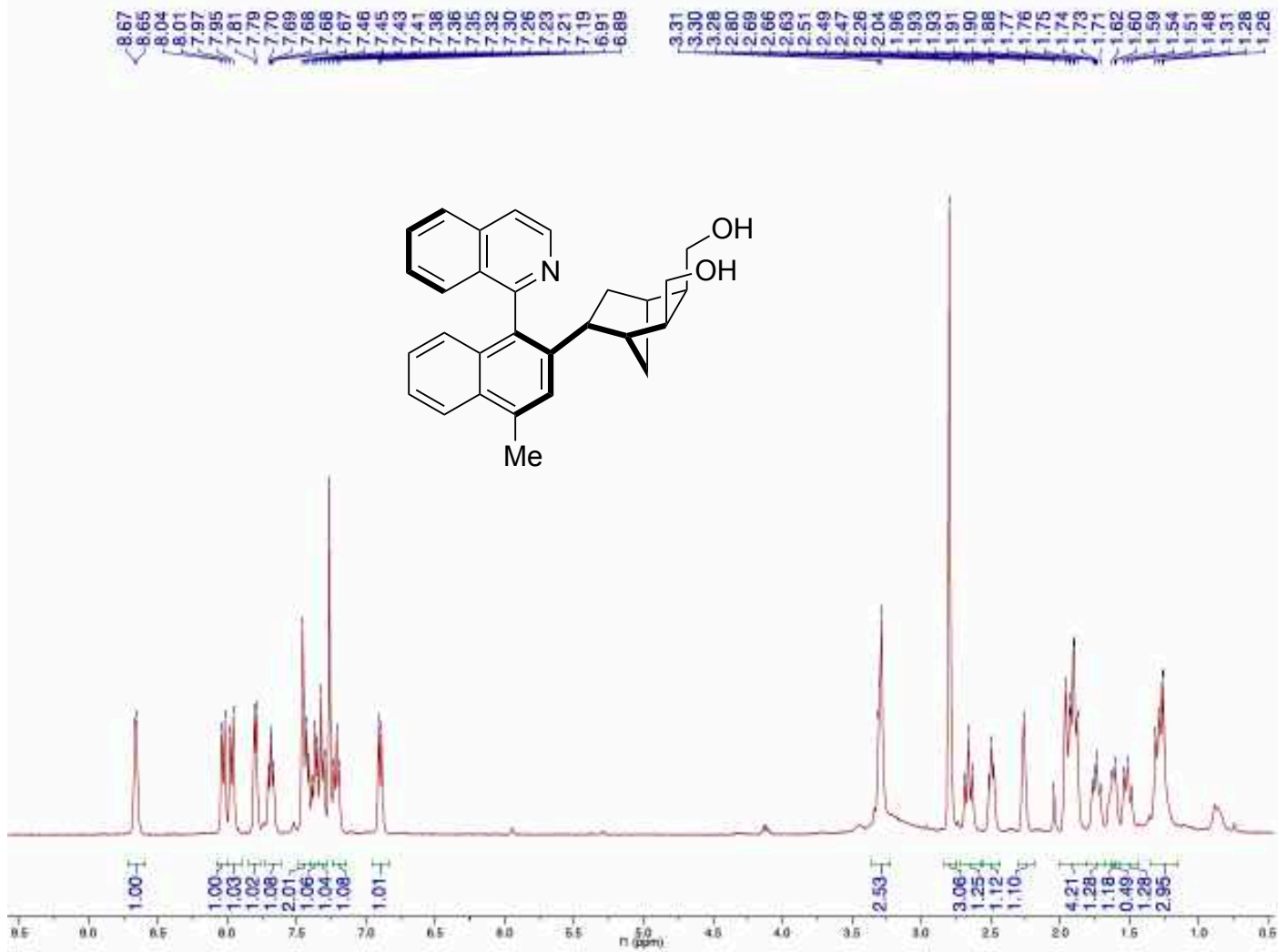

${ }^{13} \mathrm{C}$ NMR $\left(100 \mathrm{MHz}, \mathrm{CDCl}_{3}\right)$ of $\left(R_{a}, R, R, S, R, S\right)-\mathbf{8 i}$ :

mag-
0
0
0

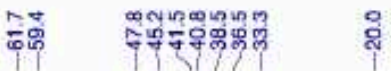<smiles>Cc1cc(C2CC3CCC2CC3CO)c(-c2nccc3ccccc23)c2ccccc12</smiles>

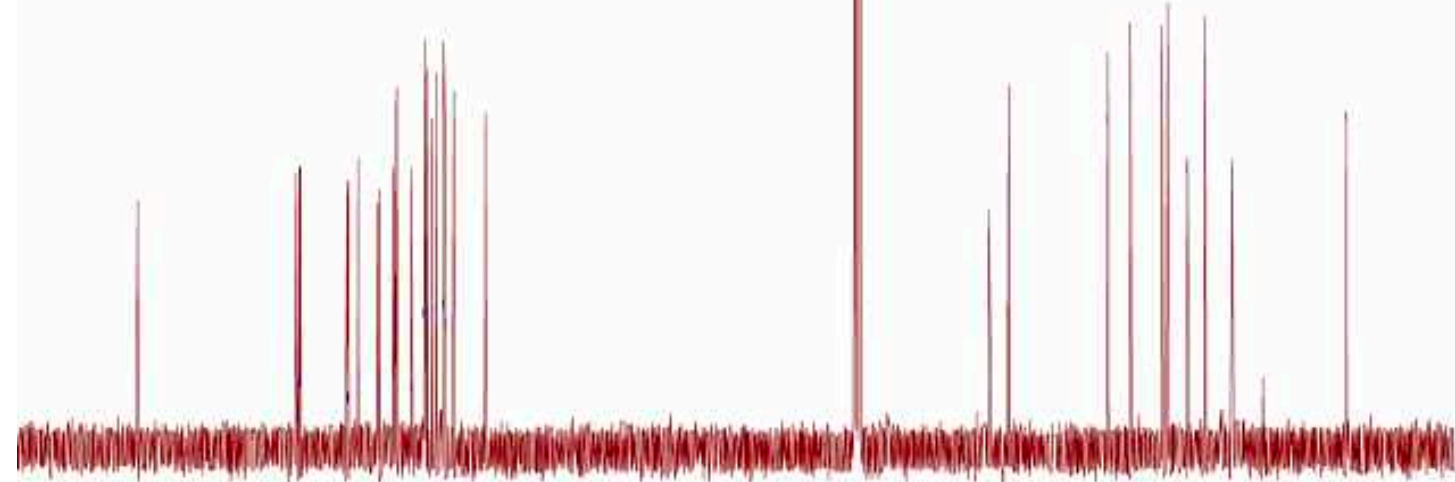


Racemic sample of $( \pm)$-8i: AS-H column, $n$-hexane:isopropanol 80:20, F= $1 \mathrm{~mL} / \mathrm{min}$, $\mathrm{T}=30^{\circ} \mathrm{C}$.

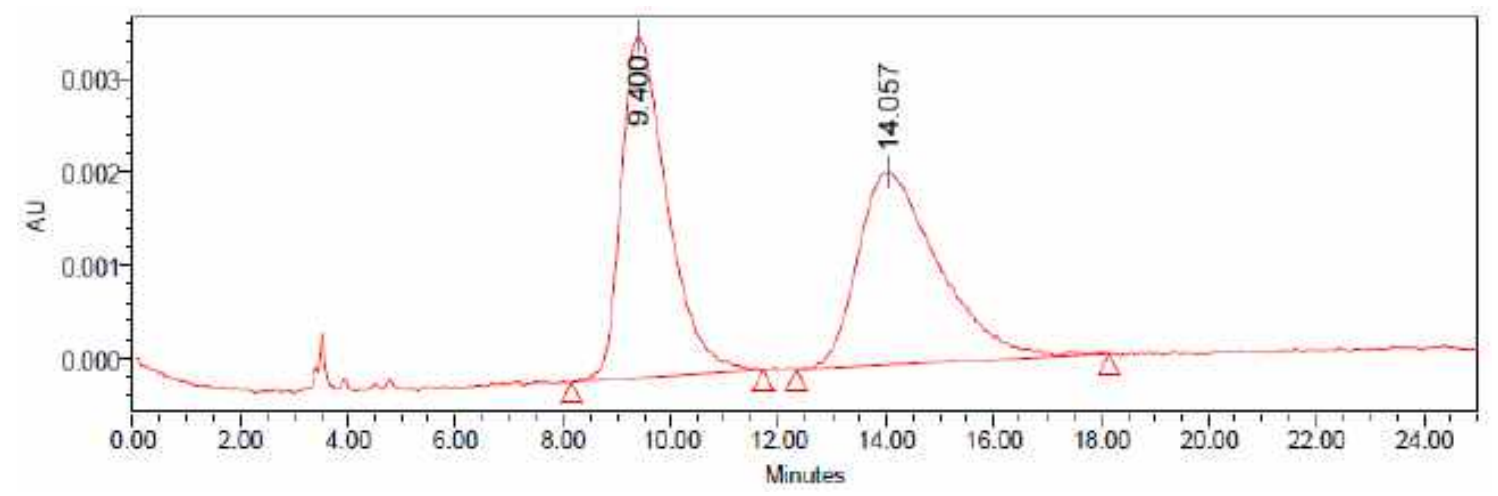

Processed Channel: PDA $230.0 \mathrm{~nm}$
\begin{tabular}{|l|l|r|r|r|r|}
\hline & Processed Channel & $\begin{array}{c}\text { Retention } \\
\text { Time (min) }\end{array}$ & Area & $\%$ Area & Height \\
\hline 1 & PDA $230.0 \mathrm{~nm}$ & 9.400 & 218728 & 50.38 & 3674 \\
\hline 2 & PDA $230.0 \mathrm{~nm}$ & 14.057 & 215446 & 49.62 & 2071 \\
\hline
\end{tabular}

Enantioriched sample of $\left(R_{a}, R, R, S, R, S\right)-8 \mathbf{i}$ :

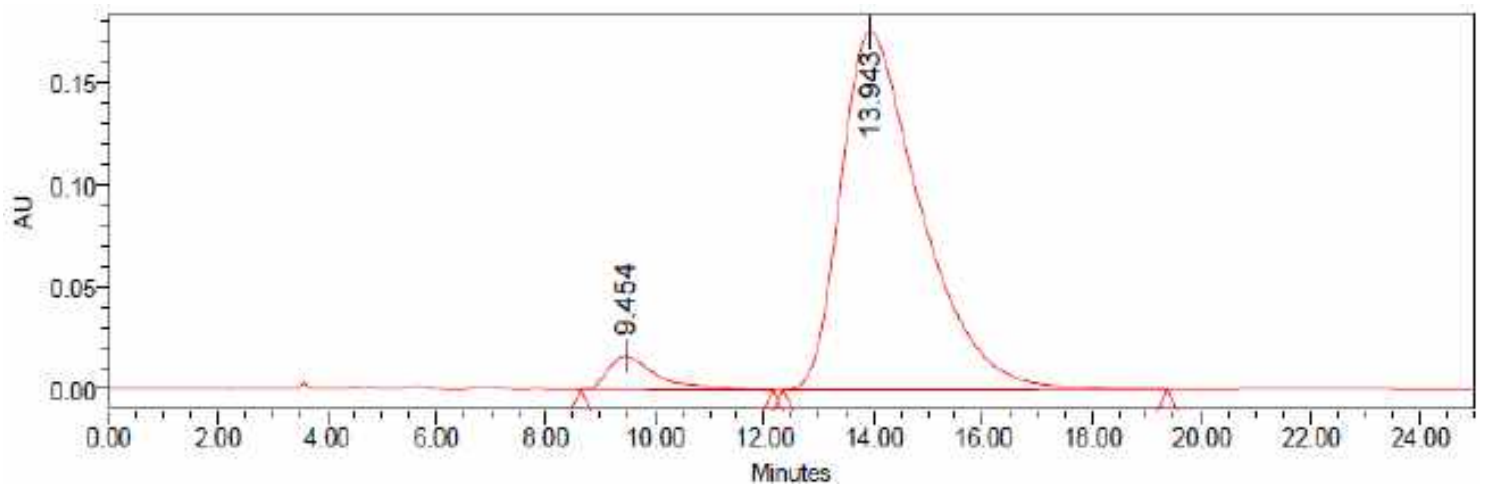

Processed Channel: PDA $230.0 \mathrm{~nm}$

\begin{tabular}{|l|l|r|r|r|r|}
\hline & Processed Channel & $\begin{array}{r}\text { Retention } \\
\text { Time }(\mathrm{min})\end{array}$ & Area & \% Area & Height \\
\hline 1 & PDA $230.0 \mathrm{~nm}$ & 9.454 & 1025360 & 5.44 & 16477 \\
\hline 2 & PDA $230.0 \mathrm{~nm}$ & 13.943 & 17833435 & 94.56 & 175028 \\
\hline
\end{tabular}


${ }^{1} \mathrm{H}$ NMR $\left(400 \mathrm{MHz}, \mathrm{CDCl}_{3}\right)$ of $\left(R_{a}, R, R, S\right)-9 \mathbf{h}:$

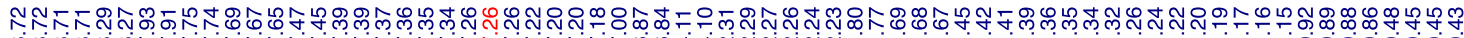

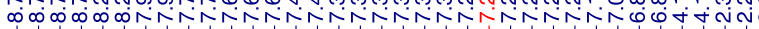

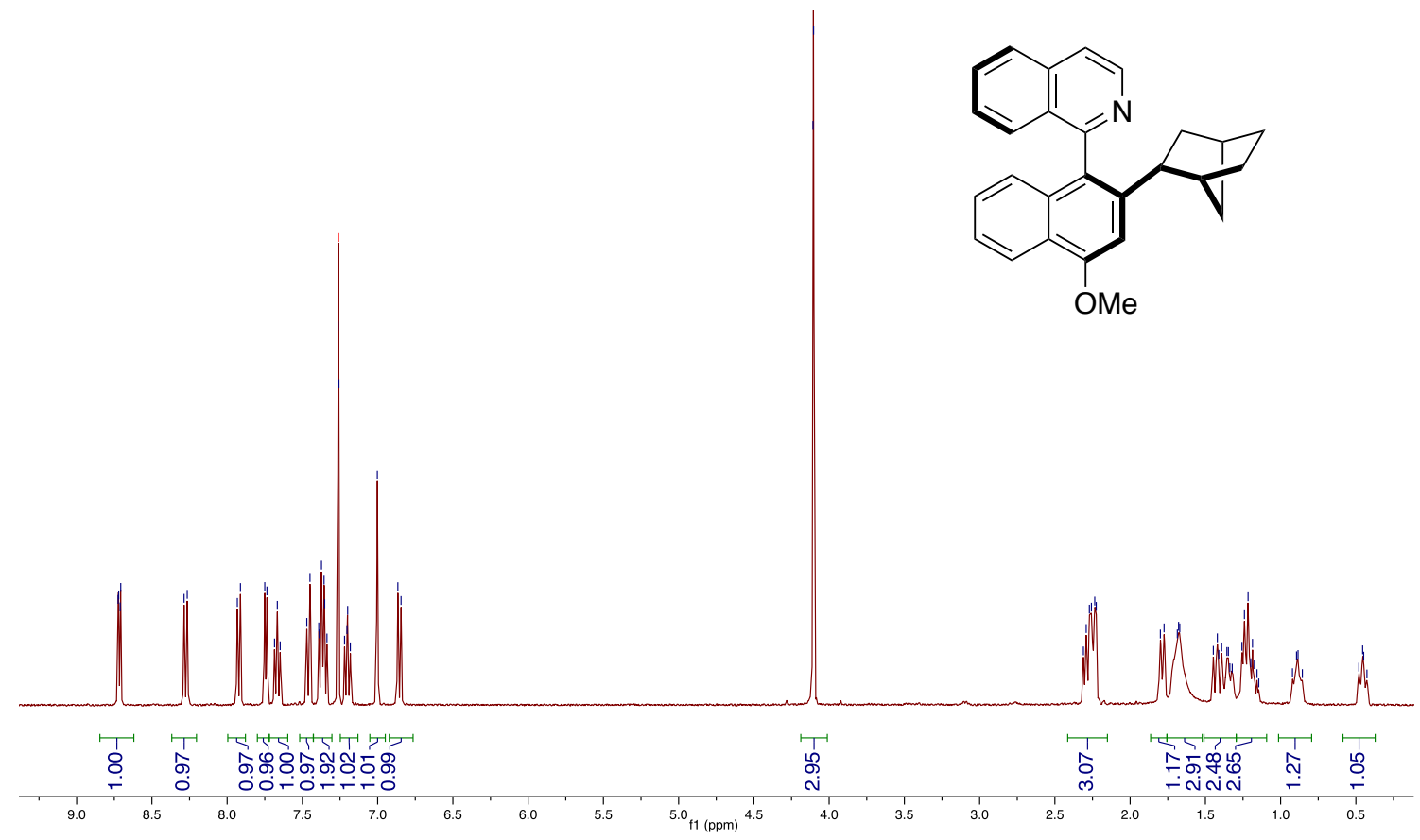

${ }^{13} \mathrm{C}$ NMR $\left(100 \mathrm{MHz}, \mathrm{CDCl}_{3}\right)$ of $\left(R_{a}, R, R, S\right)-\mathbf{9 h}$ :

일

||
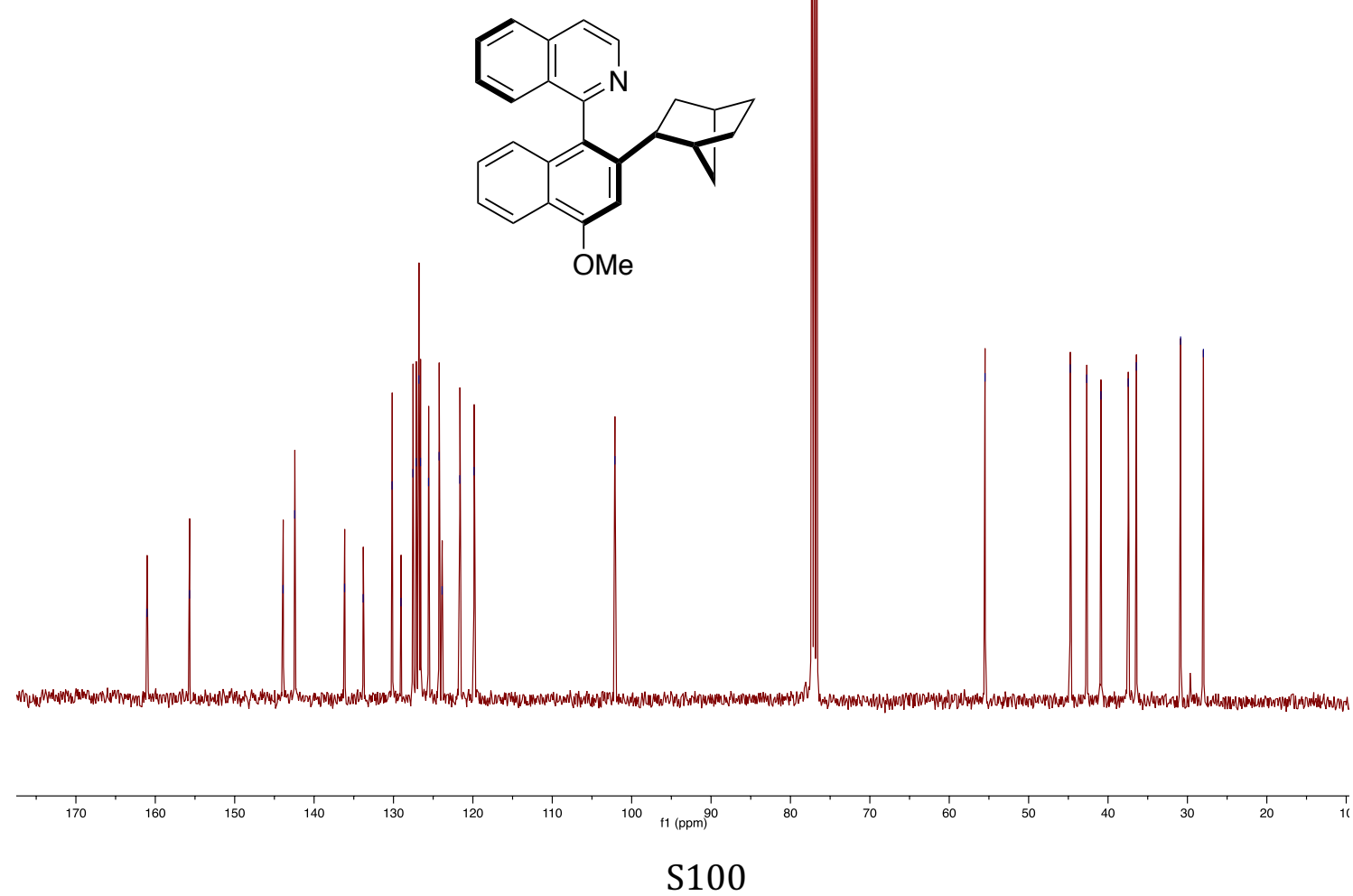
Racemic sample of $( \pm)$-9h: IA column, $n$-hexane:isopropanol 90:10, F= $1 \mathrm{~mL} / \mathrm{min}, \mathrm{T}=$ $30^{\circ} \mathrm{C}$.

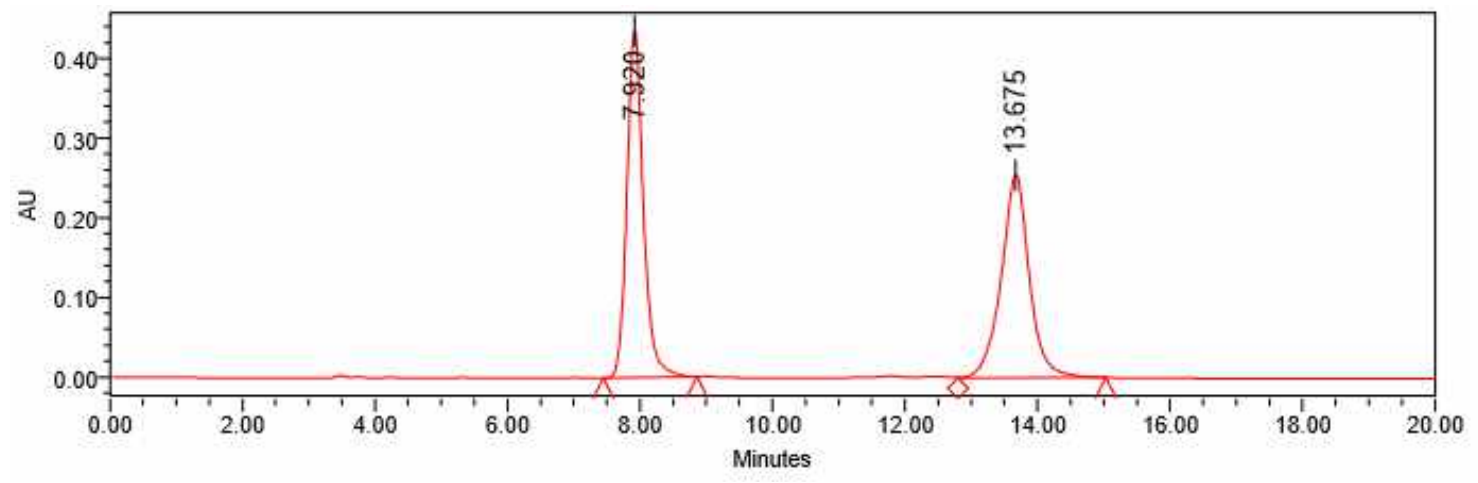

Processed Channel: PDA $218.0 \mathrm{~nm}$

\begin{tabular}{|r|l|r|c|r|r|}
\hline & Processed Channel & $\begin{array}{r}\text { Retention } \\
\text { Time (min) }\end{array}$ & Area & \% Area & Height \\
\hline 1 & PDA 218.0 nm & 7.920 & 7542254 & 50.08 & 436204 \\
\hline 2 & PDA $218.0 \mathrm{~nm}$ & 13.675 & 7518096 & 49.92 & 254878 \\
\hline
\end{tabular}

Enantioriched sample of $\left(R_{a}, R, R, S\right)$-9h:

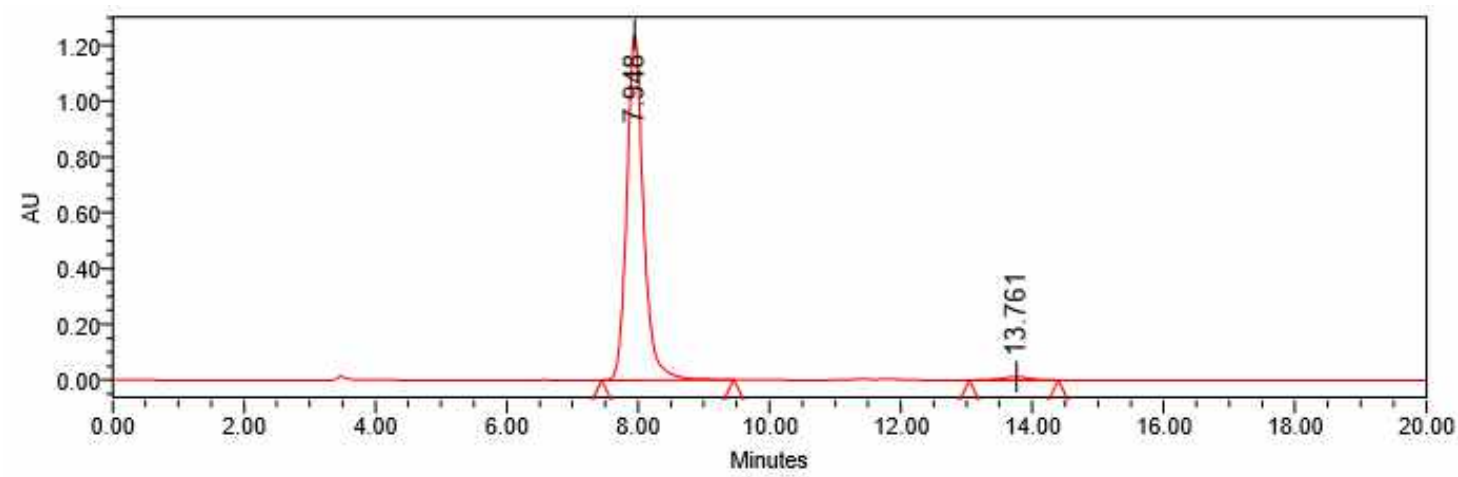

Processed Channel: PDA $218.0 \mathrm{~nm}$

\begin{tabular}{|r|l|r|r|r|r|}
\hline & Processed Channel & $\begin{array}{c}\text { Retention } \\
\text { Time (min) }\end{array}$ & Area & \% Area & Height \\
\hline 1 & PDA 218.0 nm & 7.948 & 21825214 & 98.44 & 1239577 \\
\hline 2 & PDA 218.0 nm & 13.761 & 346766 & 1.56 & 12286 \\
\hline
\end{tabular}


${ }^{1} \mathrm{H}$ NMR $\left(400 \mathrm{MHz}, \mathrm{CDCl}_{3}\right)$ of $\left(R_{a}, R, R, S, R, S\right)-9 \mathbf{i}$ :

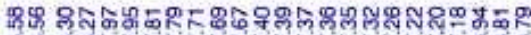

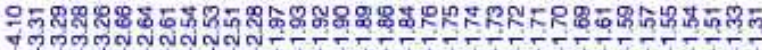
W m<smiles>COc1cc(C2CC3CCC2CC3CO)c(-c2nccc3ccccc23)c2ccccc12</smiles>
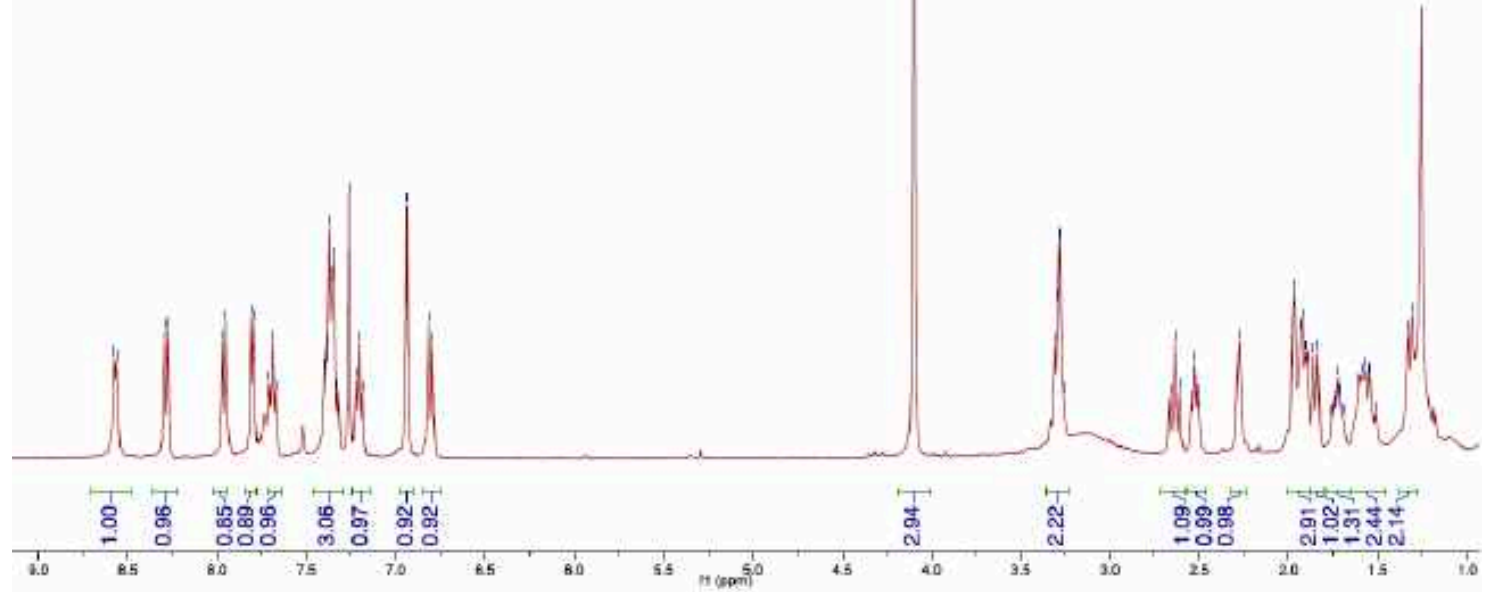

${ }^{13} \mathrm{C}$ NMR $\left(100 \mathrm{MHz}, \mathrm{CDCl}_{3}\right)$ of $\left(R_{a}, R, R, S, R, S\right)-\mathbf{9 i}$ :

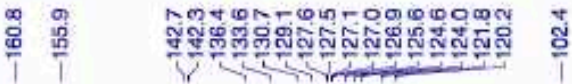

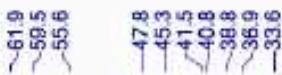
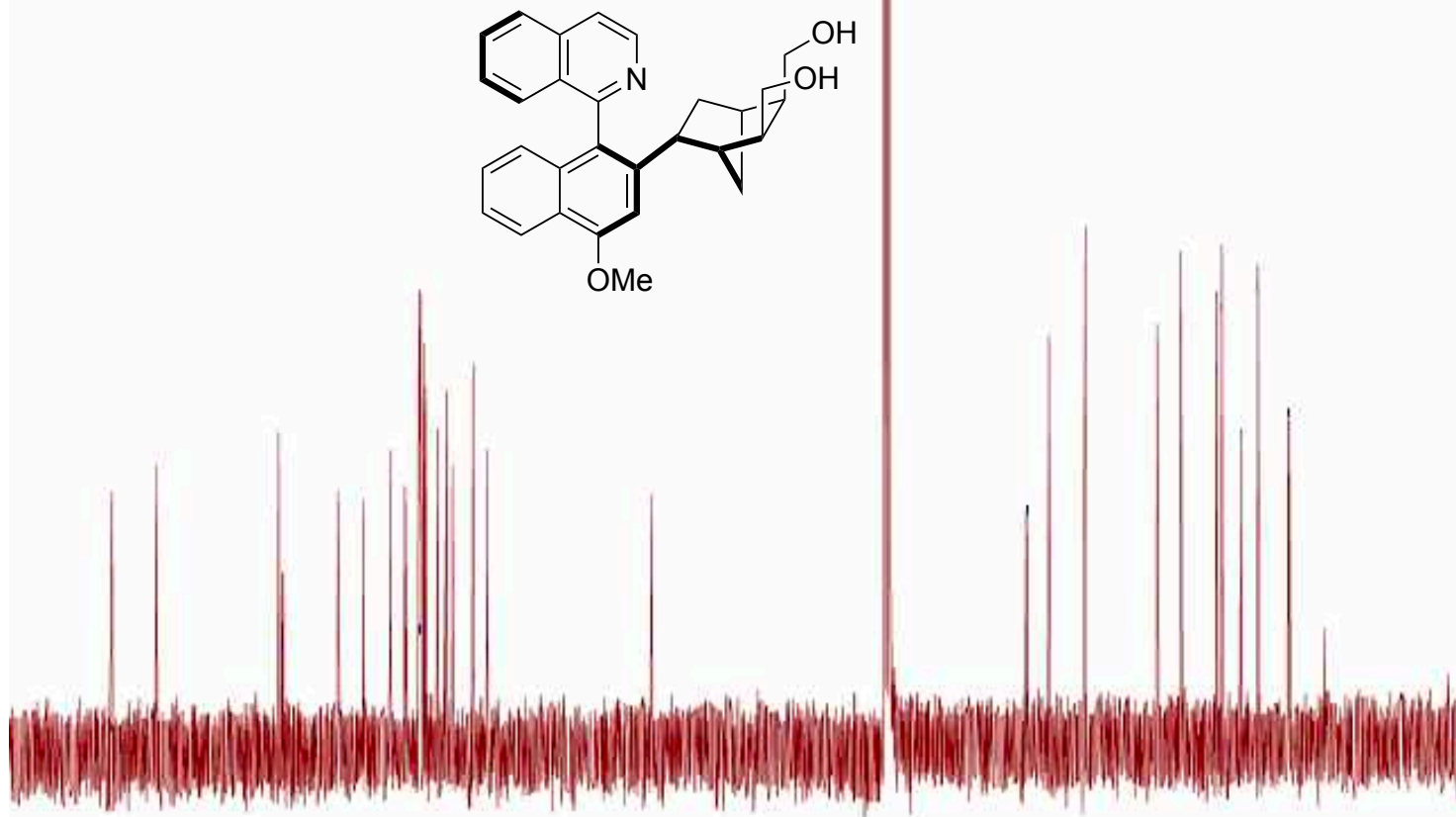

ina 
Racemic sample of $( \pm)-9 \mathrm{i}$ : AS-H column, $n$-hexane:isopropanol 80:20, $\mathrm{F}=1 \mathrm{~mL} / \mathrm{min}$, $\mathrm{T}=30^{\circ} \mathrm{C}$.

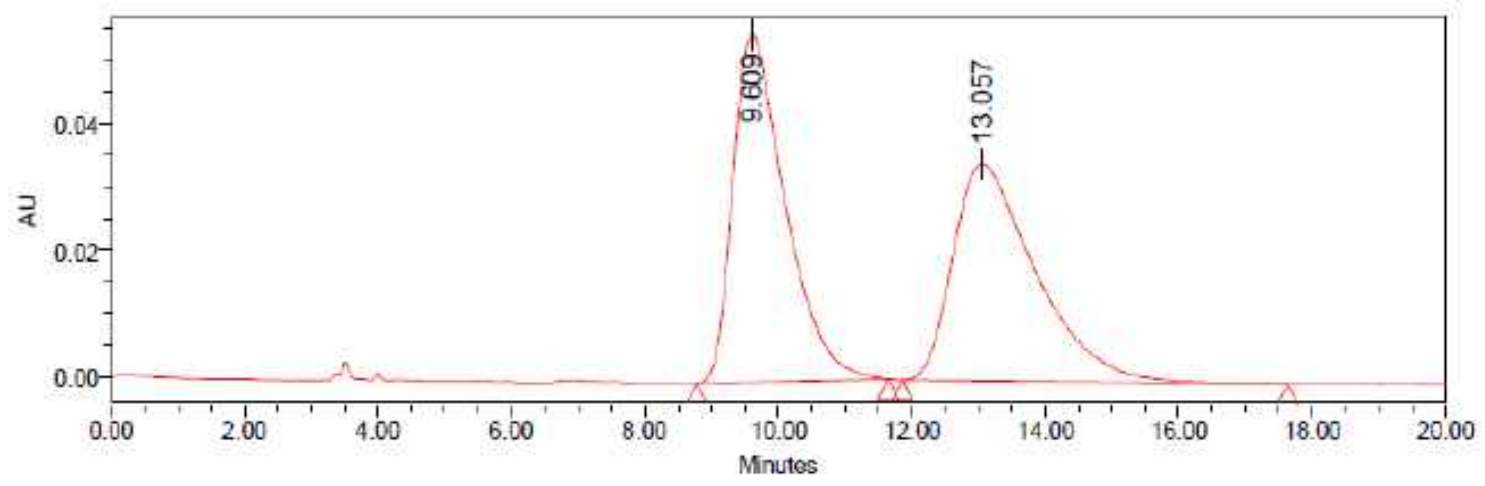

\section{Processed Channel: PDA $219.0 \mathrm{~nm}$}

\begin{tabular}{|l|l|r|r|r|r|}
\hline & Processed Channel & $\begin{array}{r}\text { Retention } \\
\text { Time (min) }\end{array}$ & Area & \% Area & Height \\
\hline 1 & PDA 219.0 nm & 9.609 & 2994685 & 50.45 & 55016 \\
\hline 2 & PDA 219.0 nm & 13.057 & 2940739 & 49.55 & 34242 \\
\hline
\end{tabular}

\section{Enantioriched sample of $\left(R_{a}, R, R, S, R, S\right)-9 \mathrm{i}$ :}

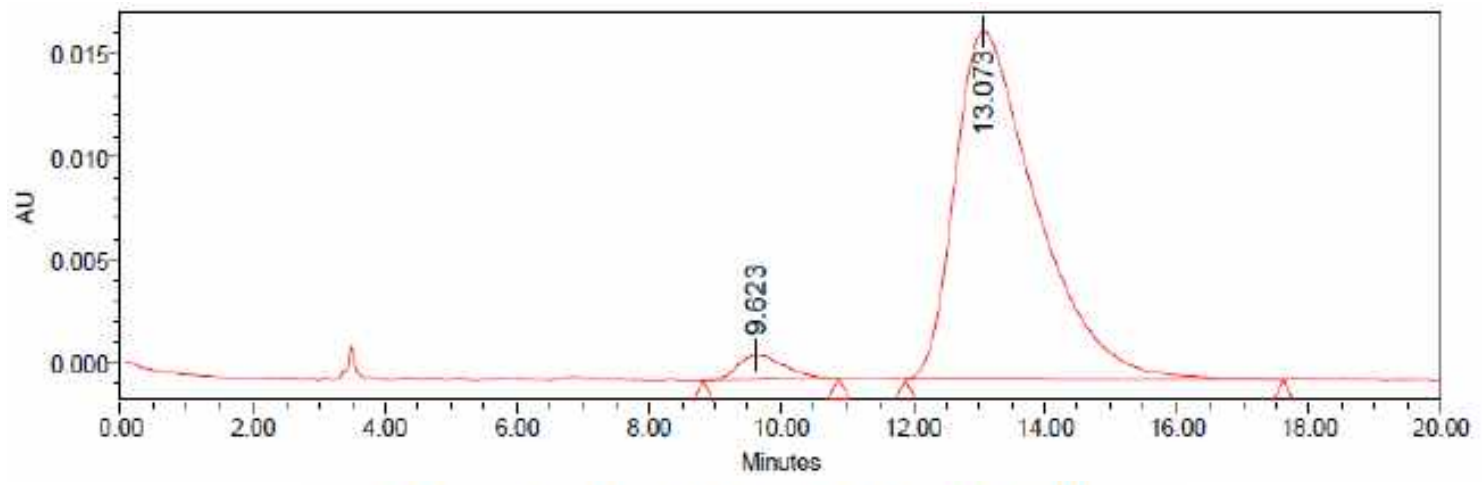

\section{Processed Channel: PDA $219.0 \mathrm{~nm}$}

\begin{tabular}{|r|l|r|r|r|r|}
\hline & Processed Channel & $\begin{array}{r}\text { Retention } \\
\text { Time }(\mathrm{min})\end{array}$ & Area & \% Area & Height \\
\hline 1 & PDA 219.0 nm & 9.623 & 60143 & 3.97 & 1204 \\
\hline 2 & PDA $219.0 \mathrm{~nm}$ & 13.073 & 1453451 & 96.03 & 16898 \\
\hline
\end{tabular}


${ }^{1} \mathrm{H}$ NMR $\left(400 \mathrm{MHz}, \mathrm{CDCl}_{3}\right)$ of $\left(R_{a}, R, R, S\right)-\mathbf{1 0 h}$ :
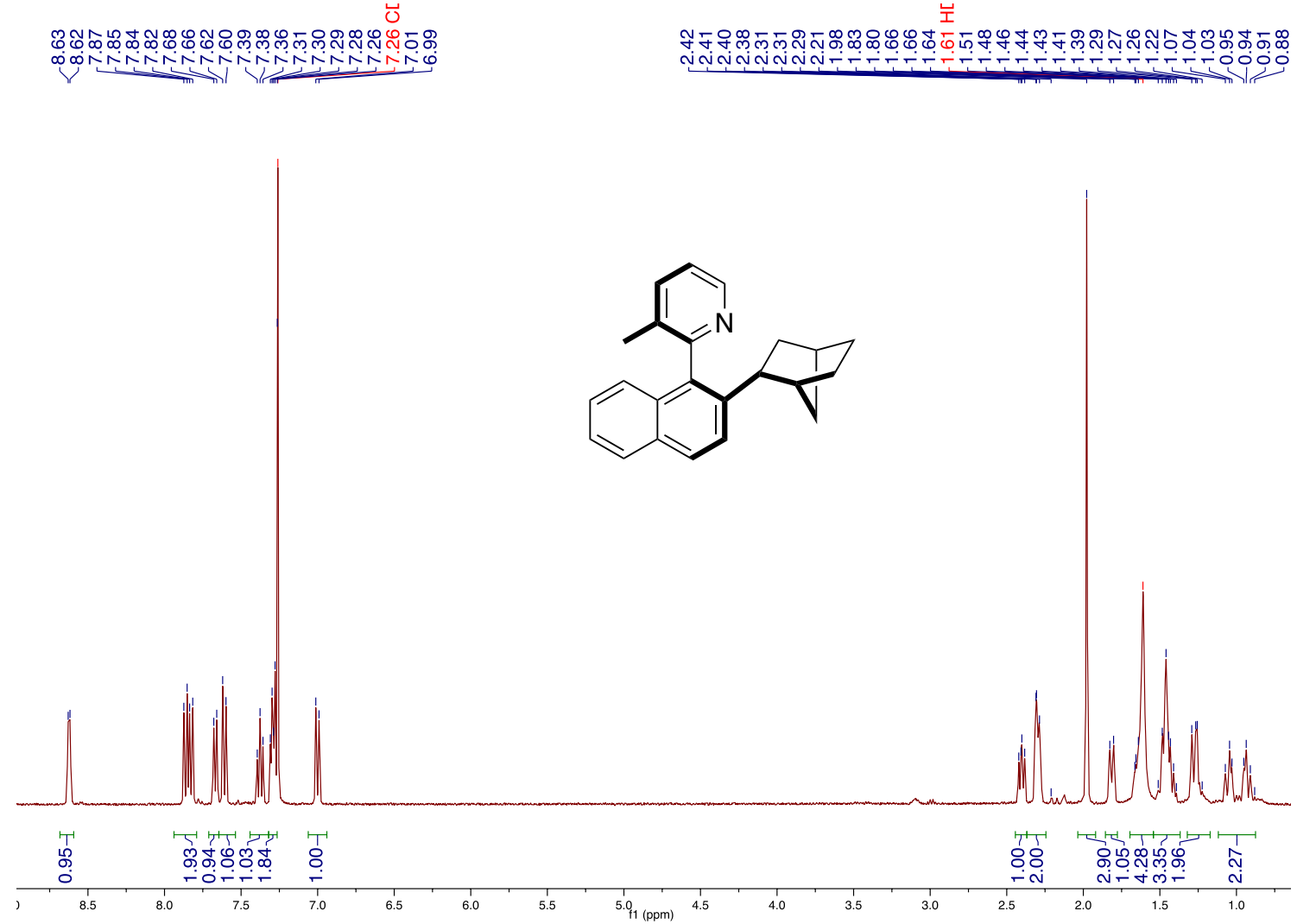

${ }^{13} \mathrm{C}$ NMR $\left(100 \mathrm{MHz}, \mathrm{CDCl}_{3}\right)$ of $\left(R_{a}, R, R, S\right)-\mathbf{1 0 h}$ :

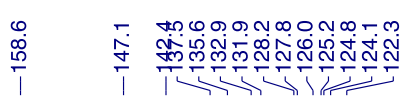

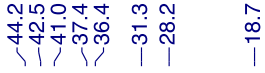

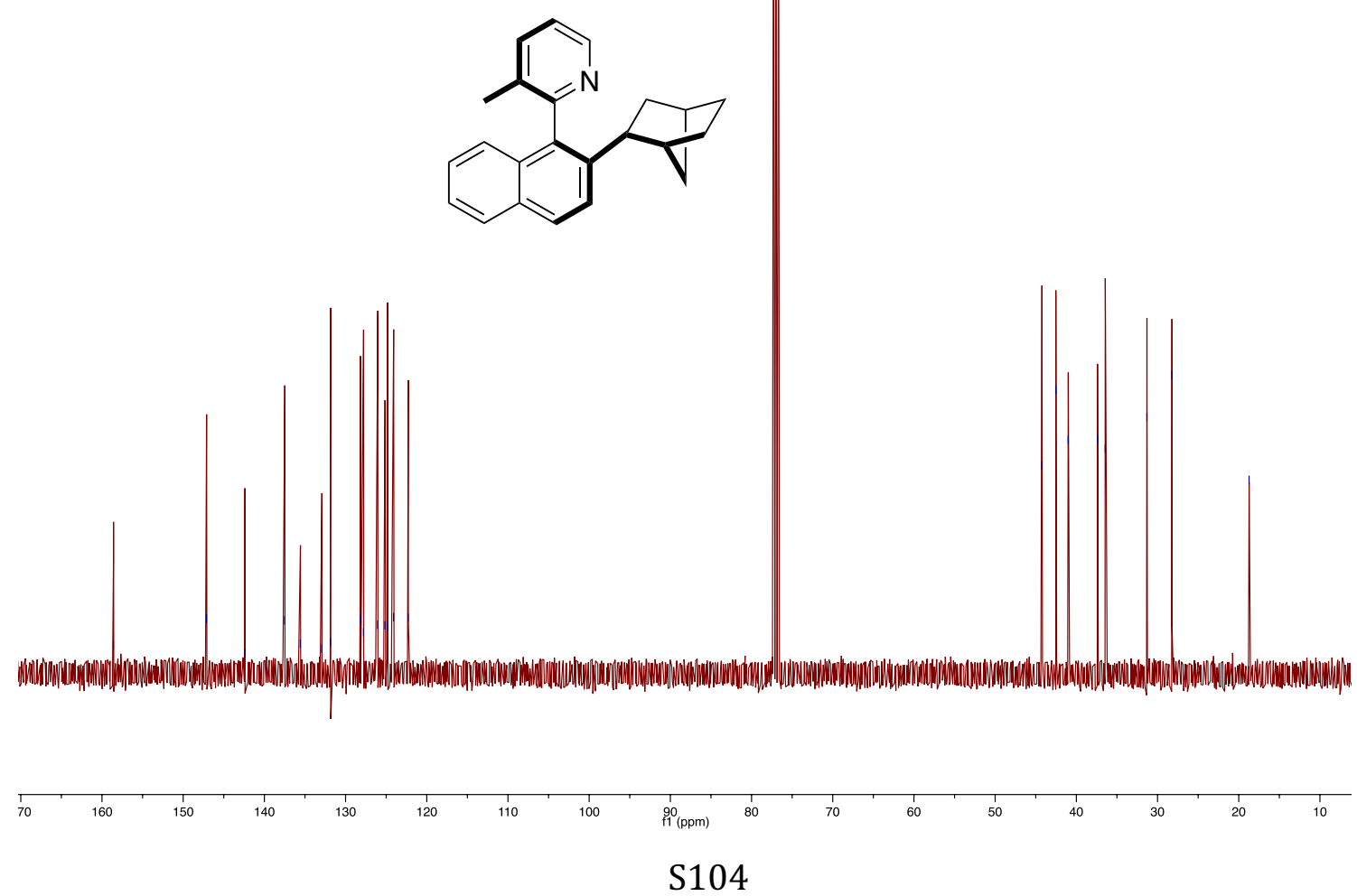


Racemic sample of $( \pm)$-10h: OD column, $n$-hexane:isopropanol 99:1, F= $1 \mathrm{~mL} / \mathrm{min}, \mathrm{T}=$ $30^{\circ} \mathrm{C}$

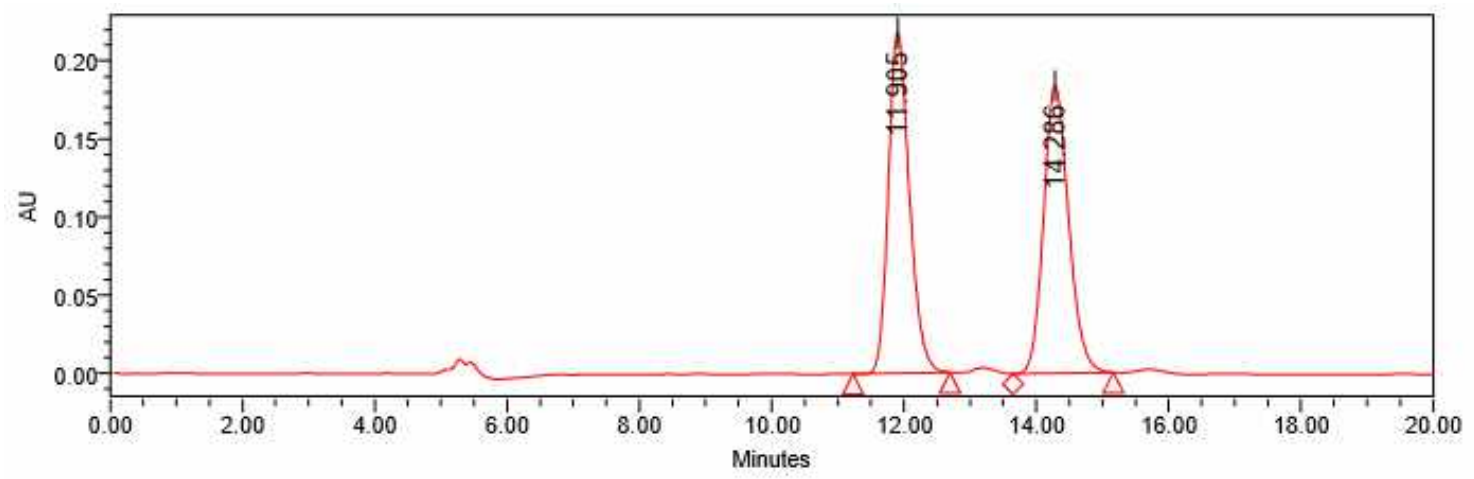

Processed Channel: PDA $227.0 \mathrm{~nm}$

\begin{tabular}{|l|l|r|c|r|r|}
\hline & Processed Channel & $\begin{array}{c}\text { Retention } \\
\text { Time (min) }\end{array}$ & Area & \% Area & Height \\
\hline 1 & PDA $227.0 \mathrm{~nm}$ & 11.905 & 4830919 & 49.94 & 218168 \\
\hline 2 & PDA $227.0 \mathrm{~nm}$ & 14.286 & 4841813 & 50.06 & 184057 \\
\hline
\end{tabular}

Enantioriched sample of $\left(R_{a}, R, R, S\right)-10 \mathrm{~h}$ :

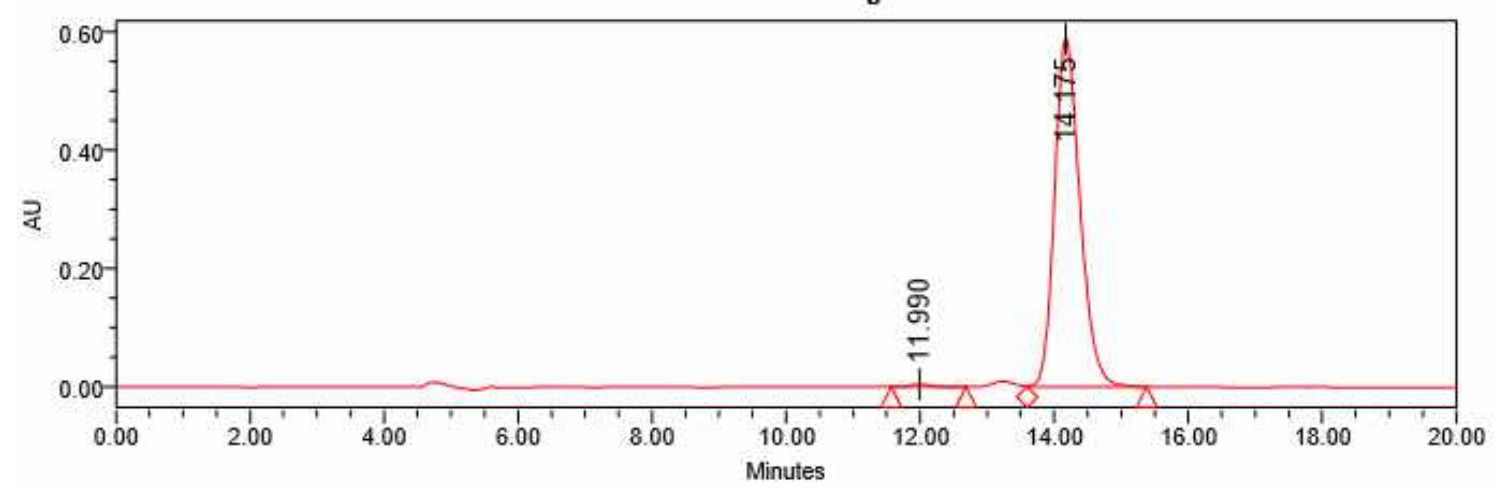

Processed Channel: PDA $227.0 \mathrm{~nm}$

\begin{tabular}{|l|l|r|r|r|r|}
\hline & Processed Channel & $\begin{array}{r}\text { Retention } \\
\text { Time (min) }\end{array}$ & Area & \% Area & Height \\
\hline 1 & PDA 227.0 nm & 11.990 & 103483 & 0.66 & 4696 \\
\hline 2 & PDA $227.0 \mathrm{~nm}$ & 14.175 & 15488488 & 99.34 & 588396 \\
\hline
\end{tabular}


${ }^{1} \mathrm{H}$ NMR $\left(400 \mathrm{MHz}, \mathrm{CDCl}_{3}\right)$ of $\left(R_{a}, R, R, S, R, S\right)-\mathbf{1 0 i}$ :

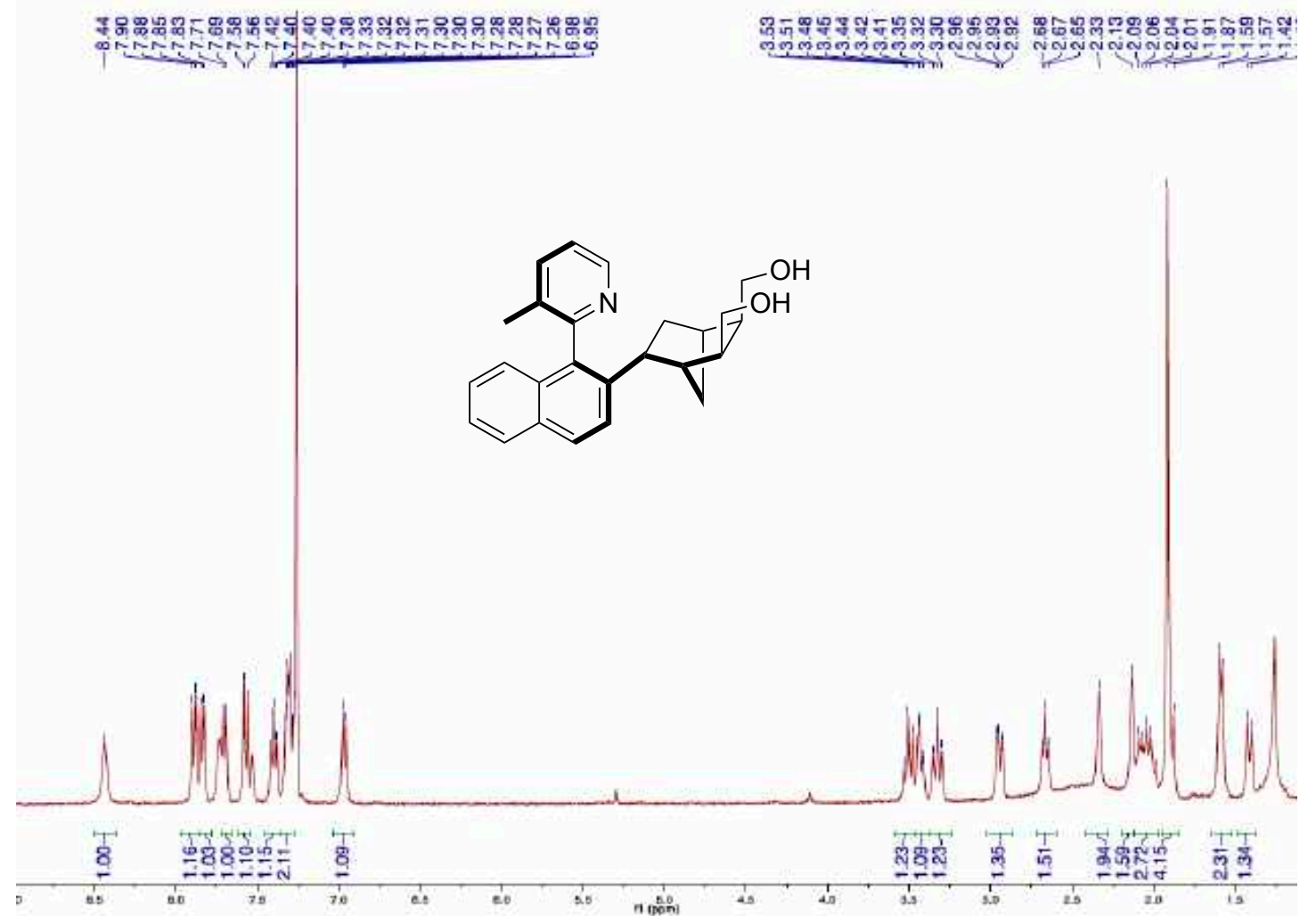

${ }^{13} \mathrm{C}$ NMR $\left(100 \mathrm{MHz}, \mathrm{CDCl}_{3}\right)$ of $\left(R_{a}, R, R, S, R, S\right)-\mathbf{1 0 i}:$

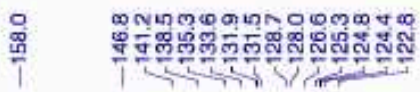

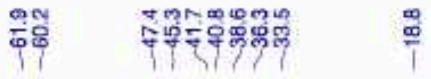<smiles>Cc1cccnc1-c1c(C2CC3CCC2CC3CO)ccc2ccccc12</smiles>

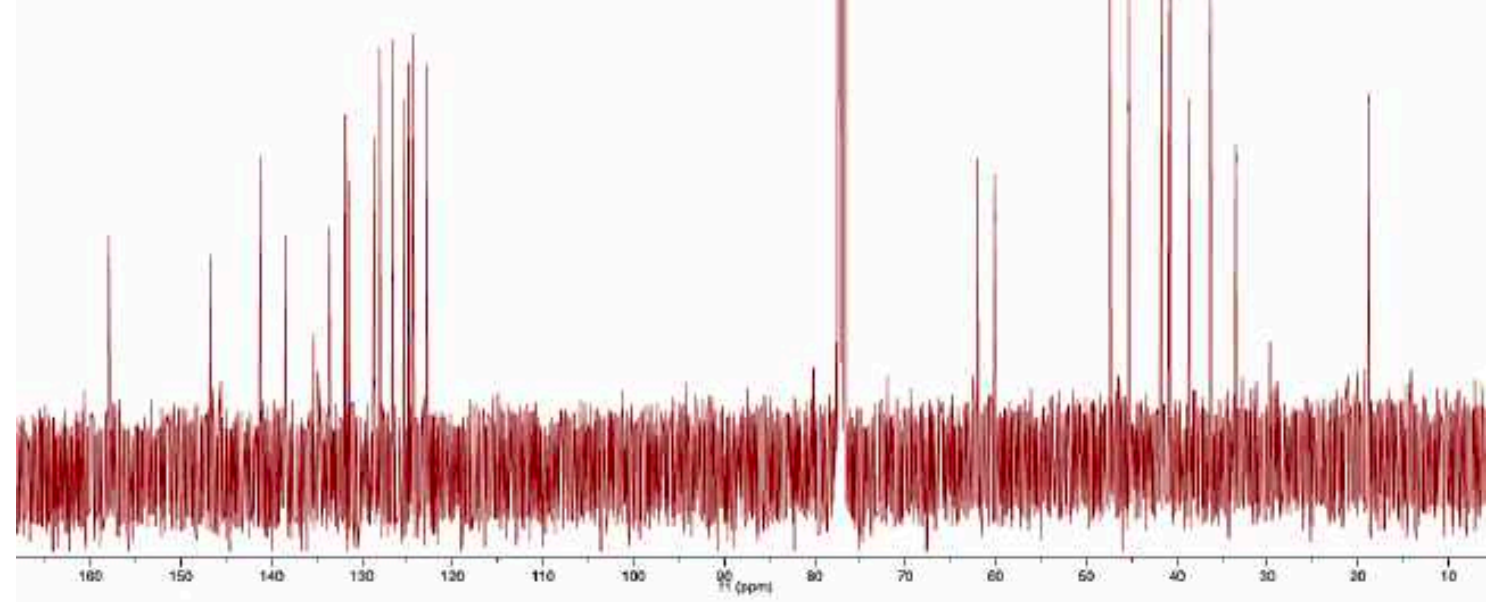


Racemic sample of $( \pm)$-10i: IA column, $n$-hexane:isopropanol 90:10, F=1 mL/min, T= $30^{\circ} \mathrm{C}$

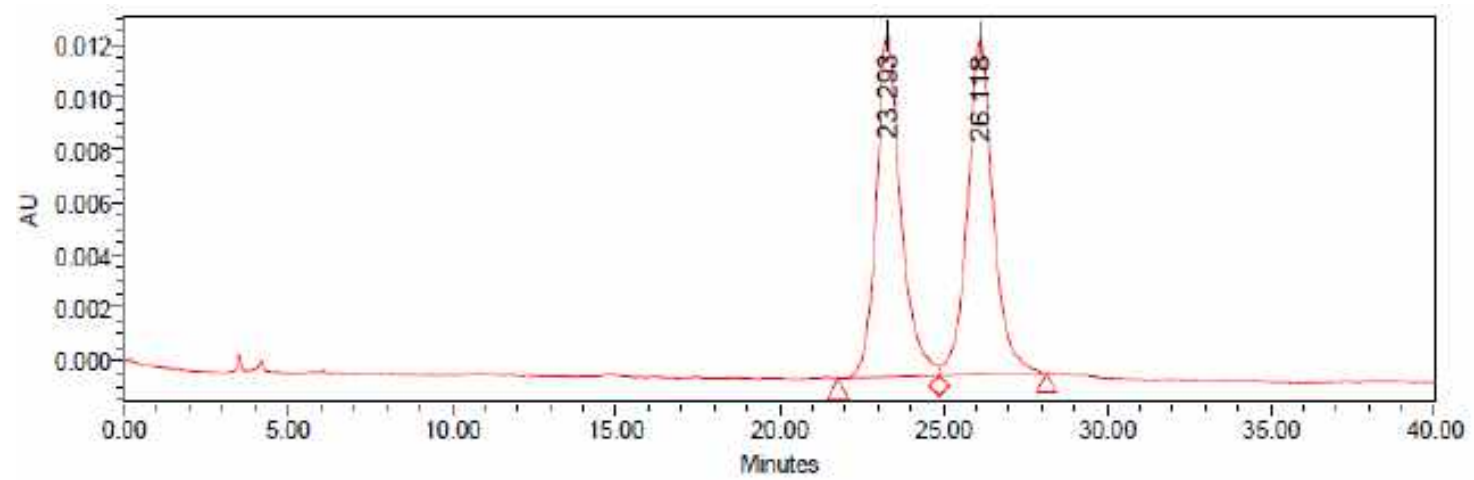

Processed Channel: PDA 227.0 nm
\begin{tabular}{|l|l|r|r|r|r|}
\hline & Processed Channel & $\begin{array}{c}\text { Retention } \\
\text { Time (min) }\end{array}$ & Area & $\%$ Area & Height \\
\hline 1 & PDA $227.0 \mathrm{~nm}$ & 23.293 & 681372 & 49.52 & 13022 \\
\hline 2 & PDA $227.0 \mathrm{~nm}$ & 26.118 & 694651 & 50.48 & 12801 \\
\hline
\end{tabular}

\section{Enantioriched sample of $\left(R_{a}, R, R, S, R, S\right)-\mathbf{1 0}$ :}

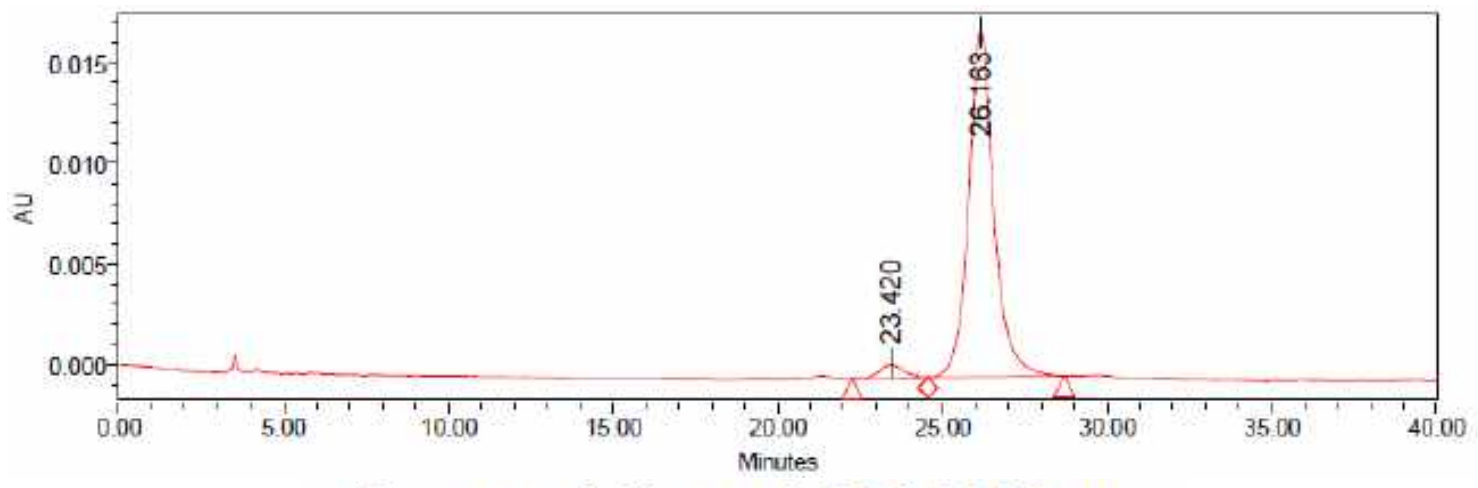

Processed Channel: PDA $227.0 \mathrm{~nm}$

\begin{tabular}{|l|l|r|r|r|r|}
\hline & Processed Channel & $\begin{array}{r}\text { Retention } \\
\text { Time }(\mathrm{min})\end{array}$ & Area & $\%$ Area & Height \\
\hline 1 & PDA $227.0 \mathrm{~nm}$ & 23.420 & 33414 & 3.52 & 666 \\
\hline 2 & PDA $227.0 \mathrm{~nm}$ & 26.163 & 915697 & 96.48 & 17115 \\
\hline
\end{tabular}


${ }^{1} \mathrm{H}$ NMR $\left(400 \mathrm{MHz}, \mathrm{CDCl}_{3}\right)$ of $\left(R_{a}, R, R, S\right)-\mathbf{1 5 h}$ :

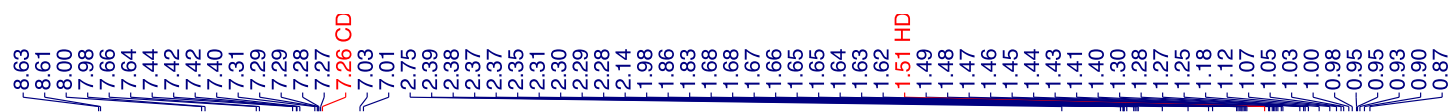<smiles>Cc1cccnc1-c1c([C@@H]2CC3CC[C@H]2C3)cc(C)c2ccccc12</smiles>
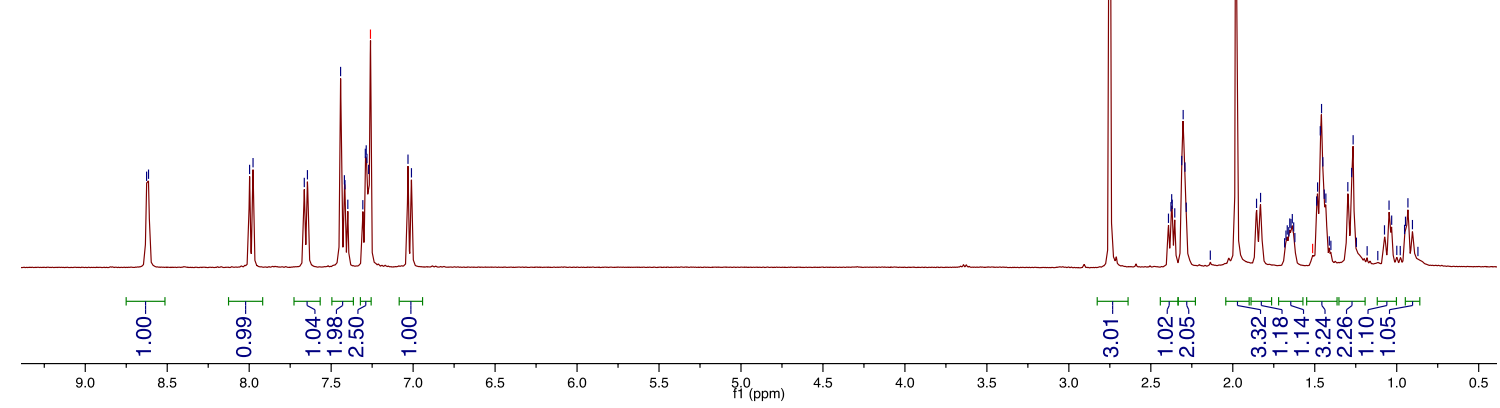

${ }^{13} \mathrm{C}$ NMR $\left(100 \mathrm{MHz}, \mathrm{CDCl}_{3}\right)$ of $\left(R_{a}, R, R, S\right)-\mathbf{1 5 h}$ :

$\frac{1}{1}$

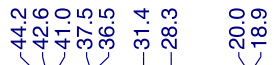

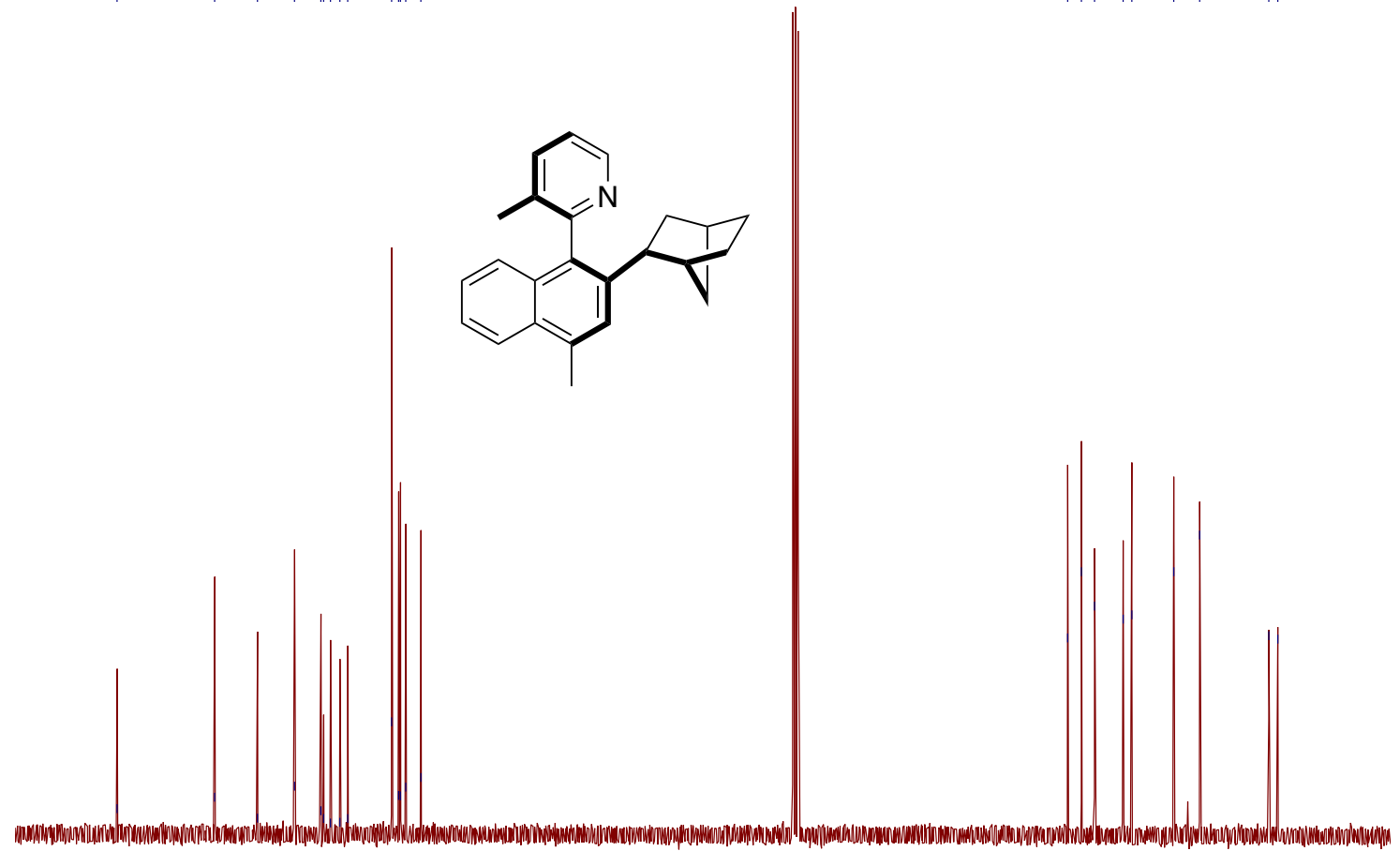

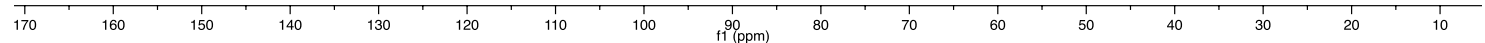


Racemic sample of $( \pm)$-15h: IA column, $n$-hexane:isopropanol 95:5, F= $1 \mathrm{~mL} / \mathrm{min}, \mathrm{T}=$ $30^{\circ} \mathrm{C}$.

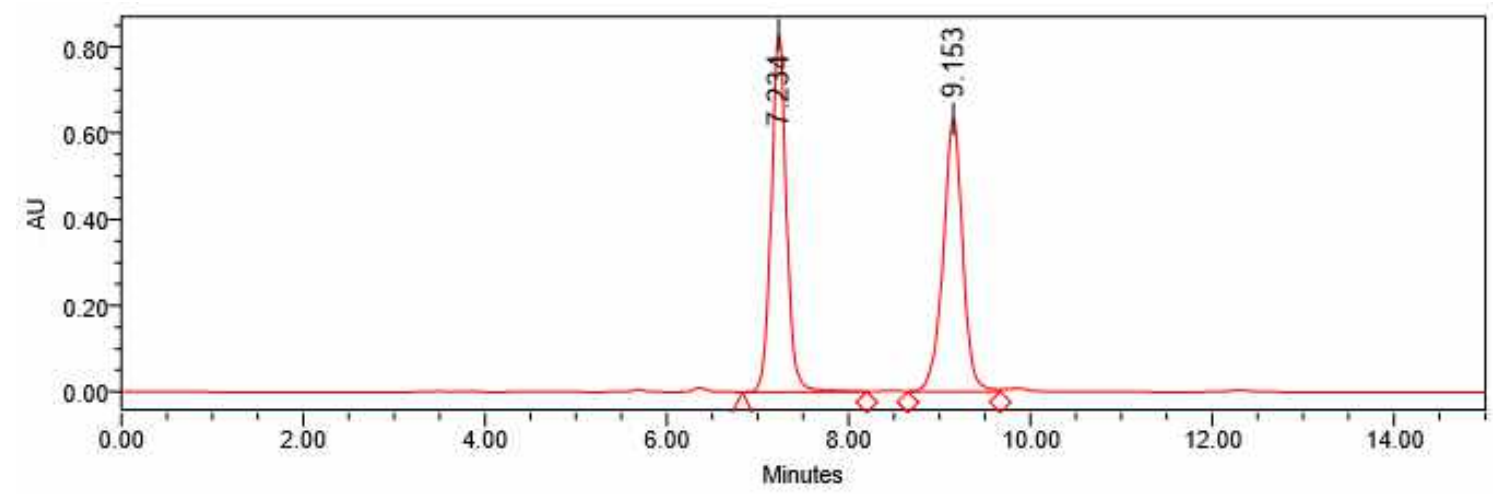

Processed Channel: PDA $232.0 \mathrm{~nm}$

\begin{tabular}{|r|l|r|r|r|r|}
\hline & Processed Channel & $\begin{array}{r}\text { Retention } \\
\text { Time (min) }\end{array}$ & Area & \% Area & Height \\
\hline 1 & PDA 232.0 nm & 7.234 & 9574000 & 50.15 & 827789 \\
\hline 2 & PDA 232.0 nm & 9.153 & 9515908 & 49.85 & 633640 \\
\hline
\end{tabular}

Enantioriched sample of $\left(R_{a}, R, R, S\right)$-15h:

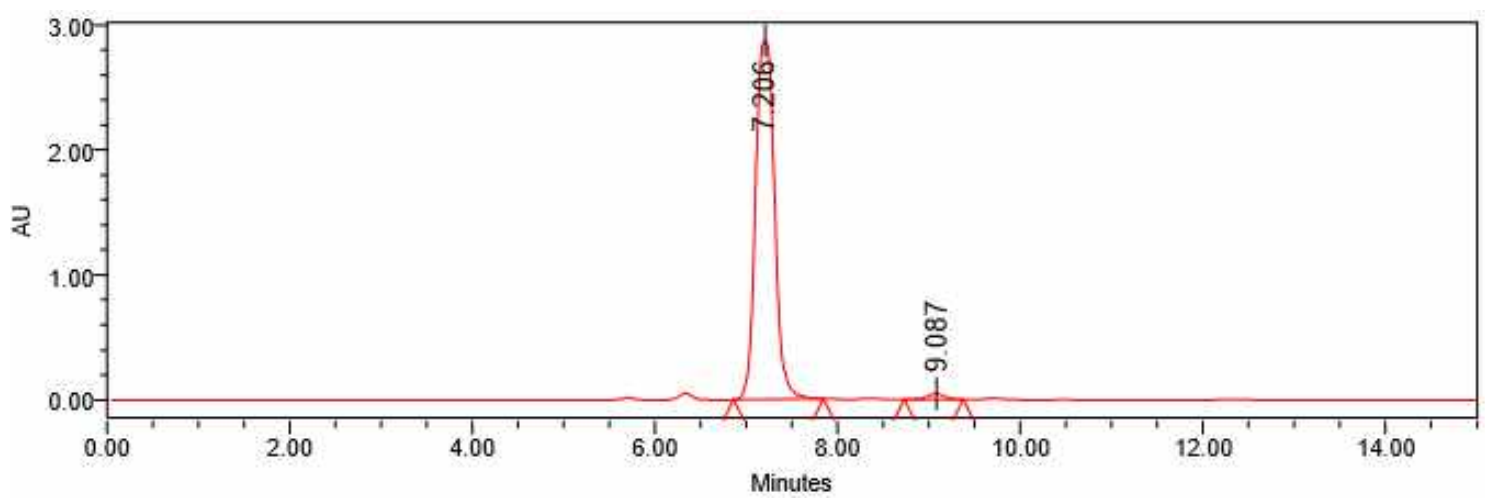

Processed Channel: PDA $232.0 \mathrm{~nm}$

\begin{tabular}{|r|l|r|r|r|r|}
\hline & Processed Channel & $\begin{array}{c}\text { Retention } \\
\text { Time }(\min )\end{array}$ & \multicolumn{1}{c|}{ Area } & \% Area & Height \\
\hline 1 & PDA 232.0 nm & 7.206 & 41365825 & 98.46 & 2873201 \\
\hline 2 & PDA 232.0 nm & 9.087 & 647980 & 1.54 & 47189 \\
\hline
\end{tabular}


${ }^{1} \mathrm{H}$ NMR $\left(400 \mathrm{MHz}, \mathrm{CDCl}_{3}\right)$ of $\left(R_{a}, R, R, S, R, S\right)-\mathbf{1 5 i}$ :

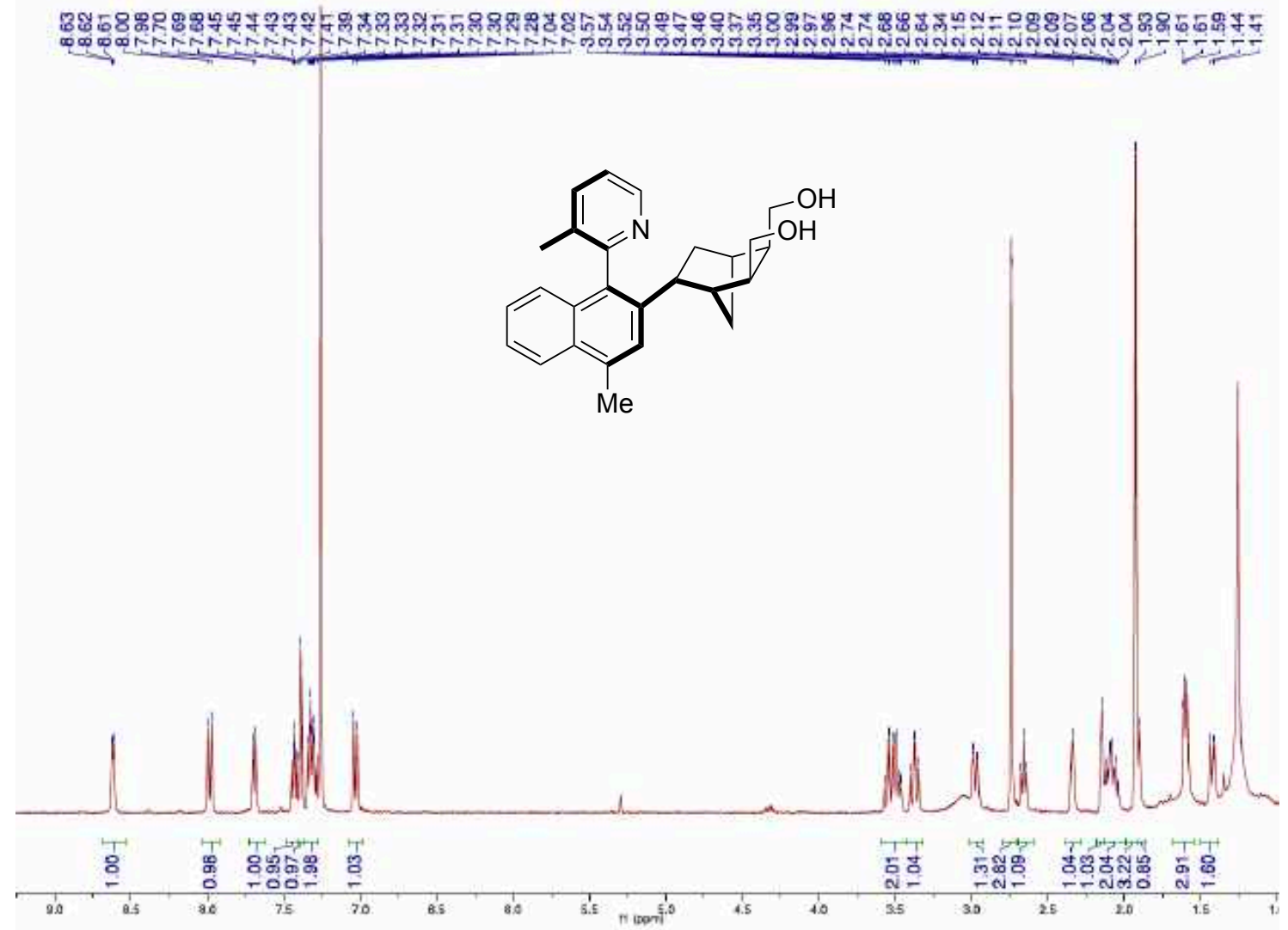

${ }^{13} \mathrm{C}$ NMR $\left(100 \mathrm{MHz}, \mathrm{CDCl}_{3}\right)$ of $\left(R_{a}, R, R, S, R, S\right)-\mathbf{1 5 i}$ :

\%

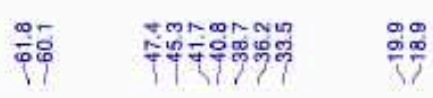<smiles>Cc1cccnc1-c1c(C2CC3CCC2CC3O)cc([N+](=O)[O-])c2ccccc12</smiles>

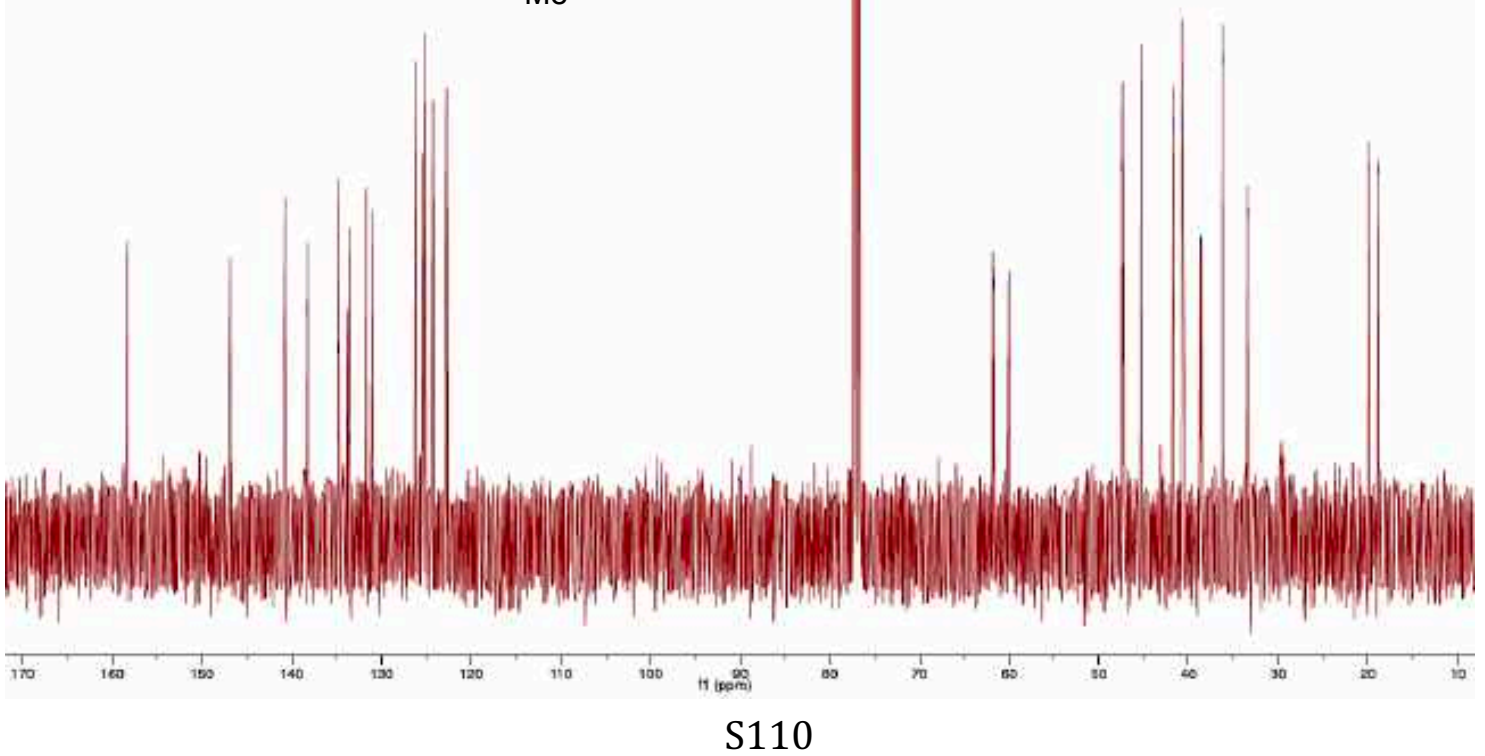


Racemic sample of $( \pm)$-15i: IC column, $n$-hexane:isopropanol 80:20, F= $1 \mathrm{~mL} / \mathrm{min}, \mathrm{T}=$ $30^{\circ} \mathrm{C}$.

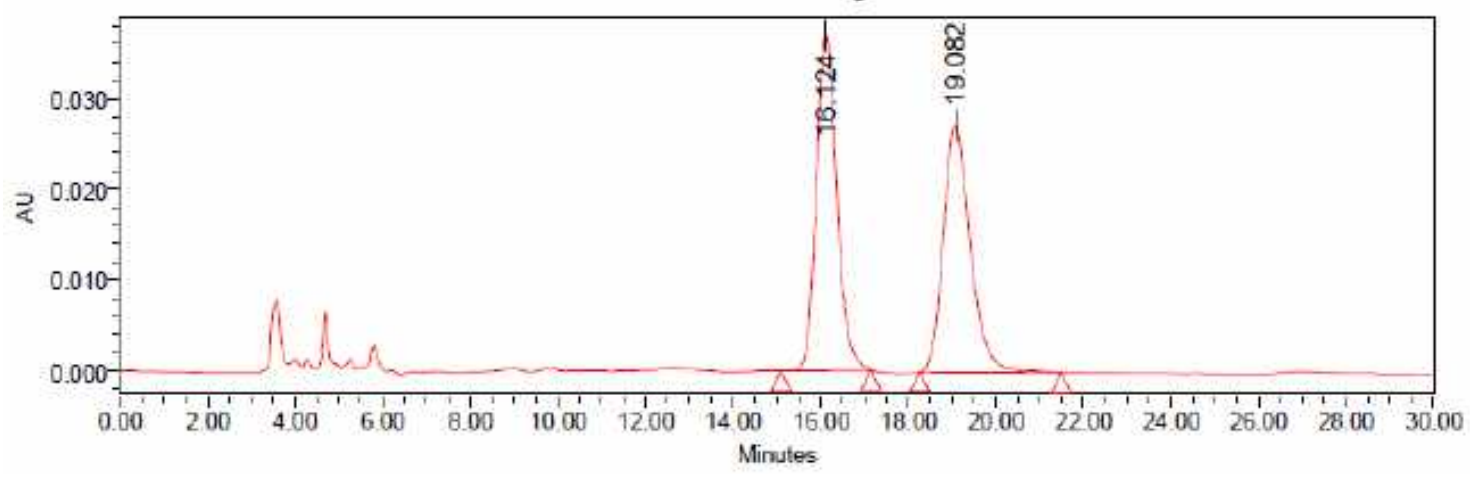

\section{Processed Channel: PDA $232.0 \mathrm{~nm}$}

\begin{tabular}{|l|l|r|r|r|r|}
\hline & Processed Channel & $\begin{array}{c}\text { Retention } \\
\text { Time }(\mathrm{min})\end{array}$ & Area & \% Area & Height \\
\hline 1 & PDA $232.0 \mathrm{~nm}$ & 16.124 & 1198493 & 50.36 & 36948 \\
\hline 2 & PDA $232.0 \mathrm{~nm}$ & 19.082 & 1181235 & 49.64 & 27263 \\
\hline
\end{tabular}

\section{Enantioriched sample of $\left(R_{a}, R, R, S, R, S\right)-\mathbf{1 5 i}:$}

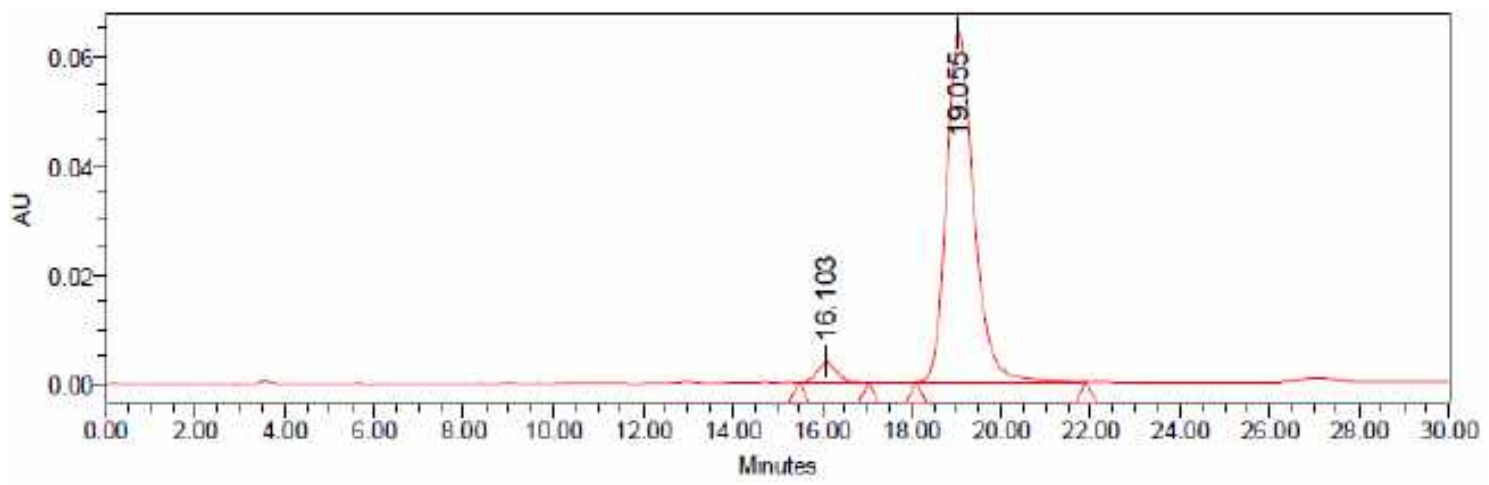

Processed Channel: PDA $232.0 \mathrm{~nm}$

\begin{tabular}{|r|l|r|r|r|r|}
\hline & Processed Channel & $\begin{array}{r}\text { Retention } \\
\text { Time (min) }\end{array}$ & Area & \% Area & Height \\
\hline 1 & PDA $232.0 \mathrm{~nm}$ & 16.103 & 110015 & 3.90 & 3690 \\
\hline 2 & PDA $232.0 \mathrm{~nm}$ & 19.055 & 2707450 & 96.10 & 64024 \\
\hline
\end{tabular}


${ }^{1} \mathrm{H} \mathrm{NMR}\left(400 \mathrm{MHz}, \mathrm{CDCl}_{3}\right)$ of $\left(R_{a}, R, R, S\right)-\mathbf{1 6 h}:$

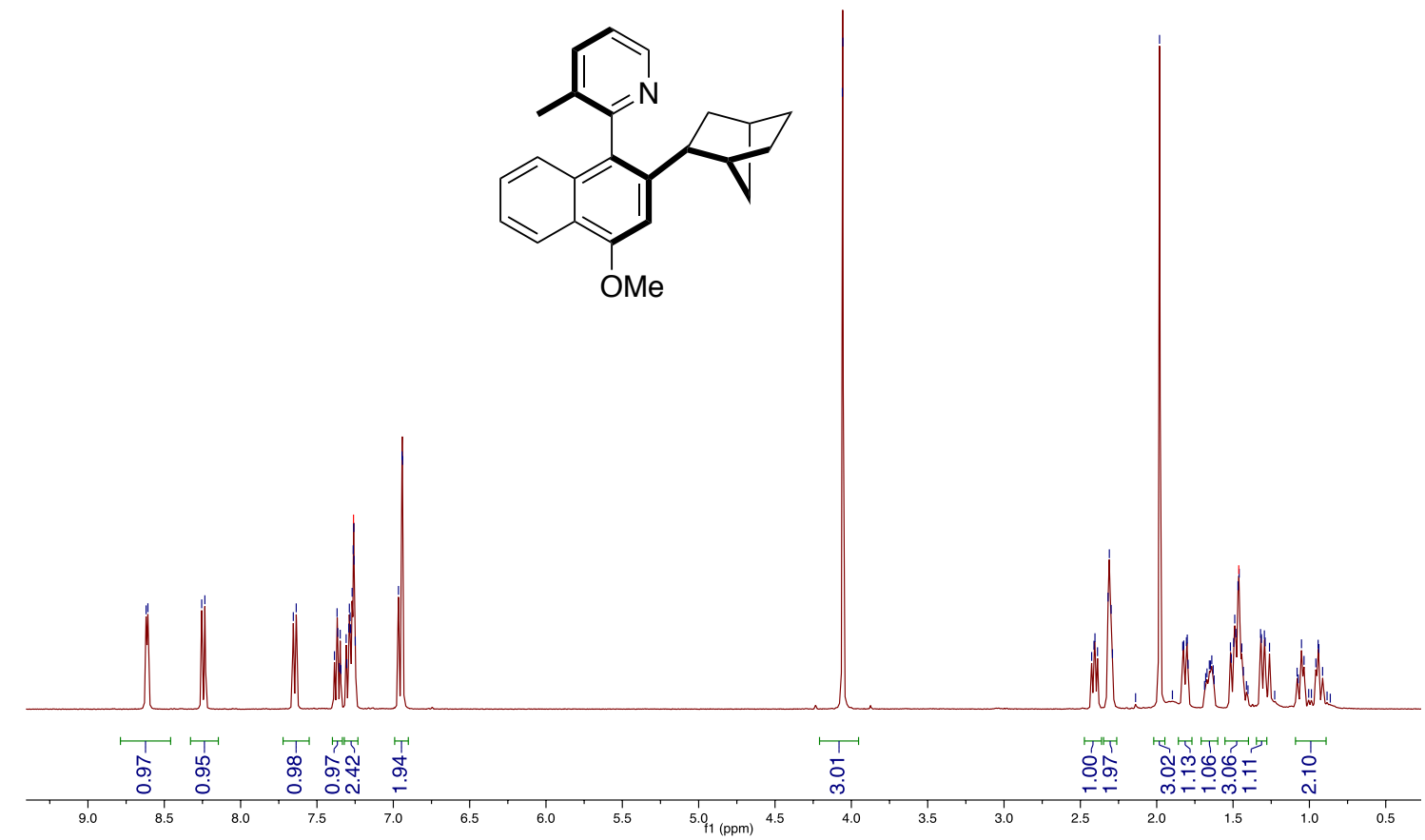

${ }^{13} \mathrm{C}$ NMR $\left(100 \mathrm{MHz}, \mathrm{CDCl}_{3}\right)$ of $\left(R_{a}, R, R, S\right)-\mathbf{1 6 h}:$

$\infty$ 舟

点

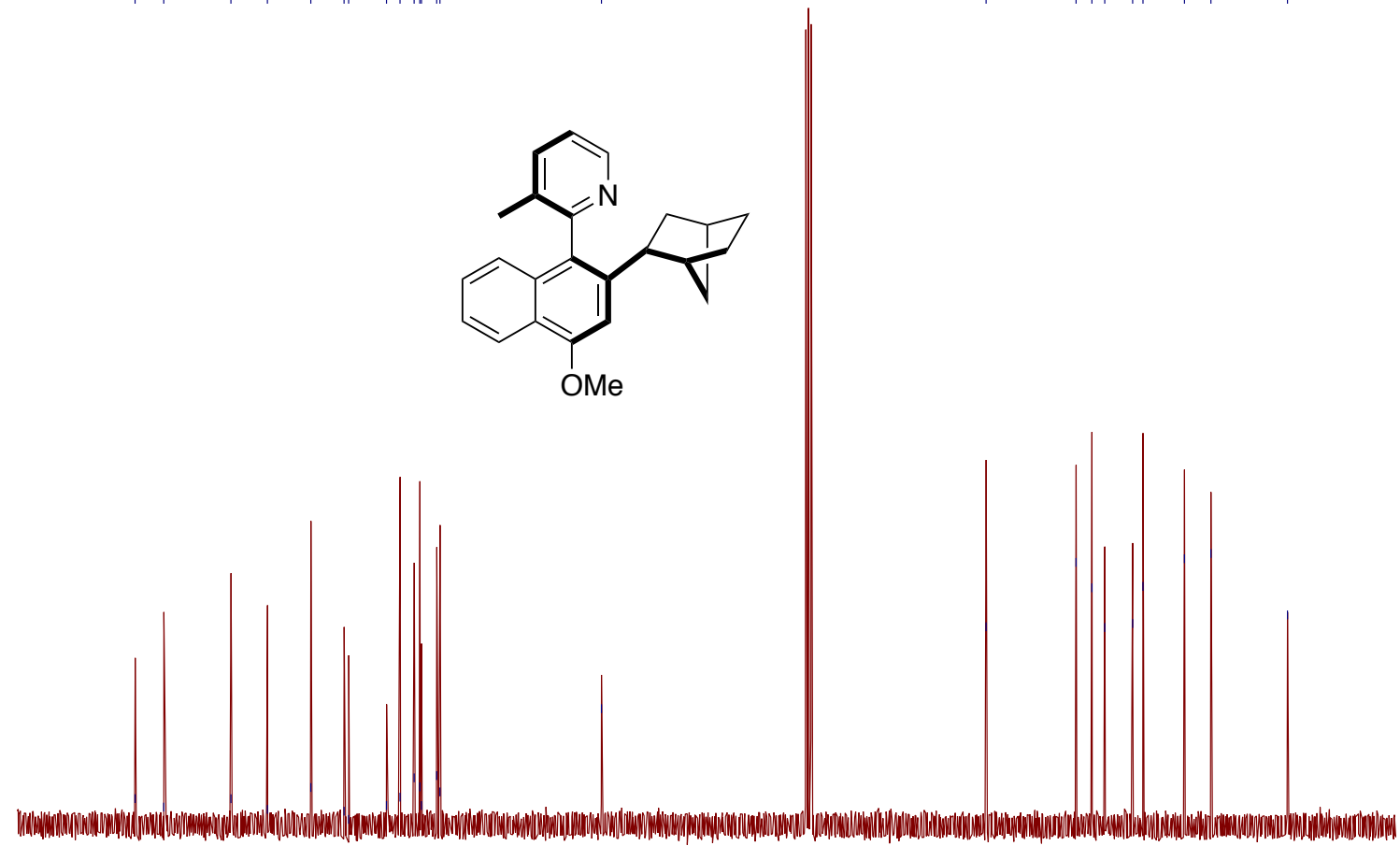

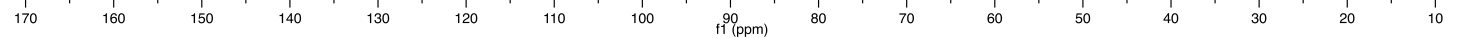


Racemic sample of $( \pm)$-16h: IA column, $n$-hexane:isopropanol 95:5, F= $1 \mathrm{~mL} / \mathrm{min}, \mathrm{T}=$ $30^{\circ} \mathrm{C}$

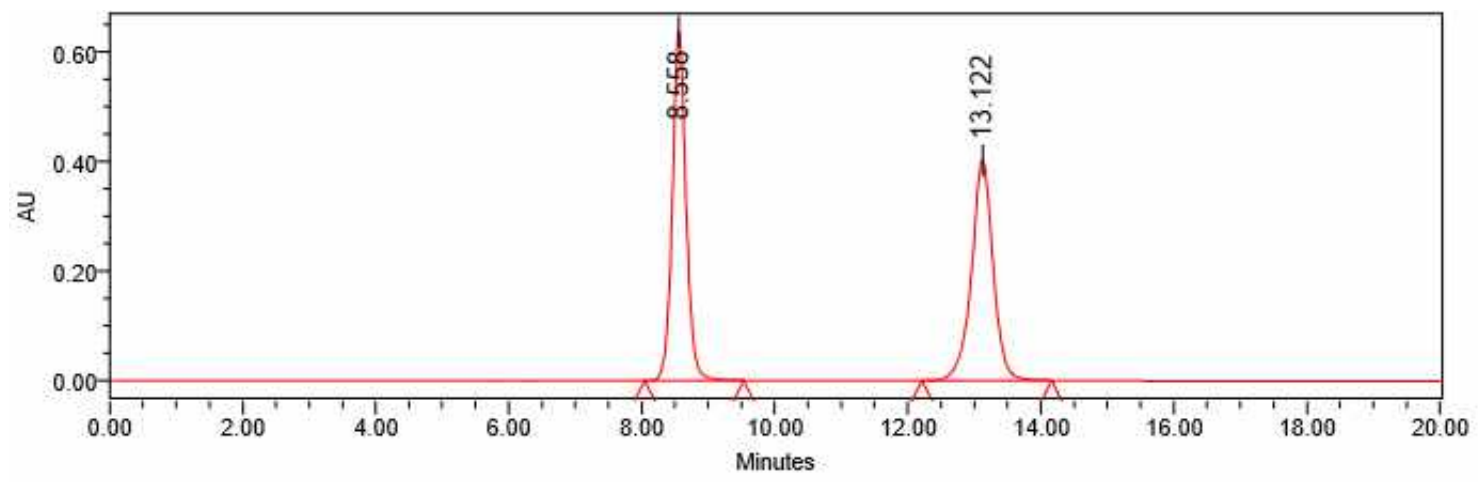

Processed Channel: PDA $236.0 \mathrm{~nm}$

\begin{tabular}{|r|l|r|c|r|r|}
\hline & Processed Channel & $\begin{array}{c}\text { Retention } \\
\text { Time (min) }\end{array}$ & Area & \% Area & Height \\
\hline 1 & PDA $236.0 \mathrm{~nm}$ & 8.558 & 9170344 & 50.10 & 640096 \\
\hline 2 & PDA $236.0 \mathrm{~nm}$ & 13.122 & 9135185 & 49.90 & 403047 \\
\hline
\end{tabular}

Enantioriched sample of $\left(R_{a}, R, R, S\right)$-16h:

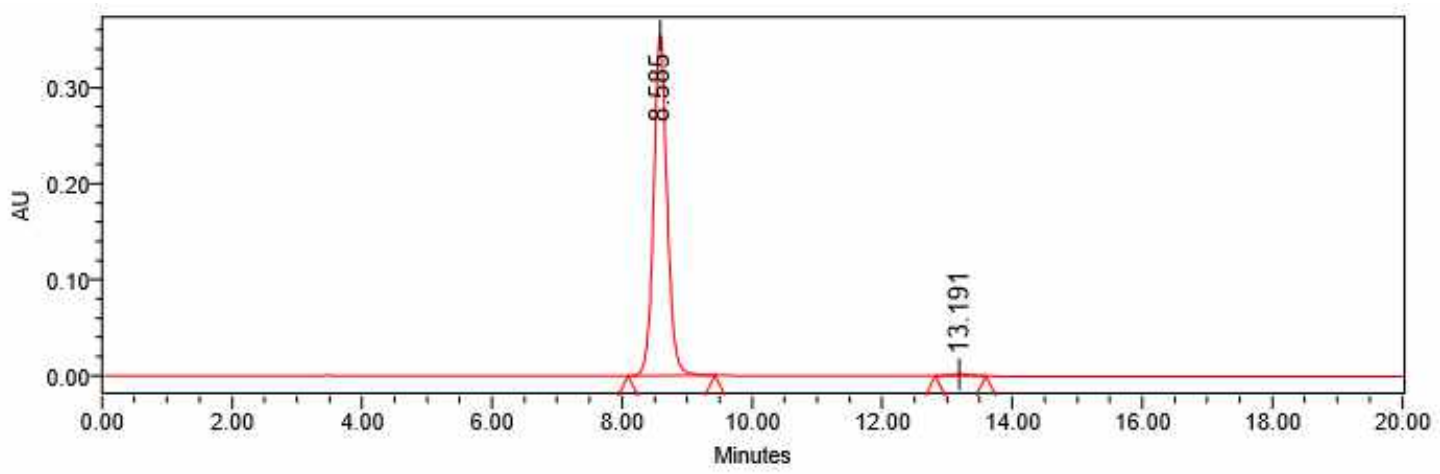

Processed Channel: PDA $236.0 \mathrm{~nm}$

\begin{tabular}{|r|l|r|r|r|r|}
\hline & Processed Channel & $\begin{array}{r}\text { Retention } \\
\text { Time (min) }\end{array}$ & Area & \% Area & Height \\
\hline 1 & PDA 236.0 nm & 8.585 & 5081947 & 99.18 & 355877 \\
\hline 2 & PDA $236.0 \mathrm{~nm}$ & 13.191 & 42248 & 0.82 & 2114 \\
\hline
\end{tabular}


${ }^{1} \mathrm{H}$ NMR $\left(400 \mathrm{MHz}, \mathrm{CDCl}_{3}\right)$ of $\left(R_{a}, R, R, S, R, S\right)-\mathbf{1 6} \mathbf{i}:$

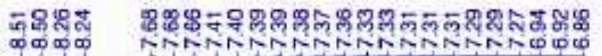

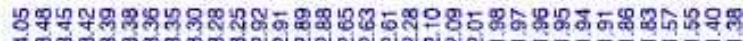

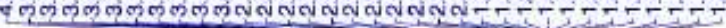<smiles>COc1cc(C2CC3CCC2CC3CO)c(-c2ncccc2C)c2ccccc12</smiles>
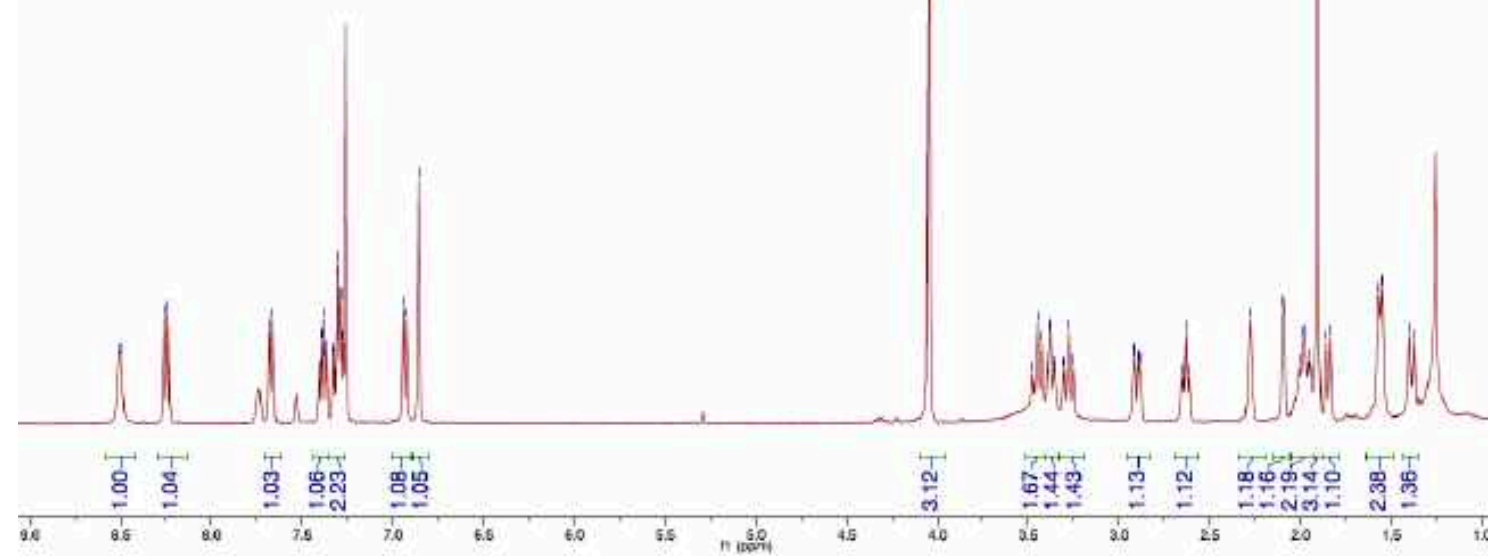

${ }^{13} \mathrm{C}$ NMR $\left(100 \mathrm{MHz}, \mathrm{CDCl}_{3}\right)$ of $\left(R_{a}, R, R, S, R, S\right)-\mathbf{1 6} \mathbf{i}:$

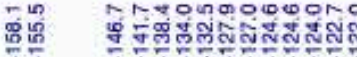

d

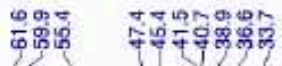

$\stackrel{\infty}{\Gamma}$<smiles>COc1cc(C2CC3CCC2CC3CO)c(-c2ncccc2C)c2ccccc12</smiles>

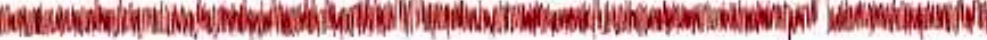
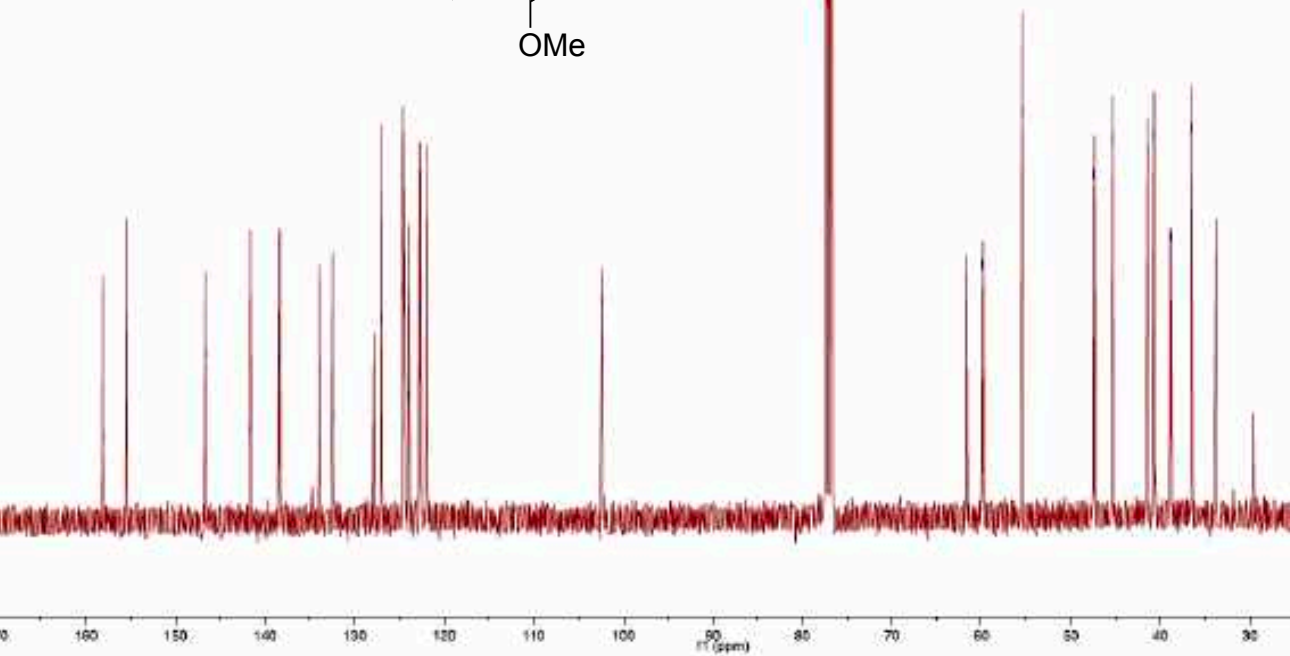
Racemic sample of $( \pm)$-16i: IA column, $n$-hexane:isopropanol 80:20, F= $1 \mathrm{~mL} / \mathrm{min}, \mathrm{T}=$ $30^{\circ} \mathrm{C}$.

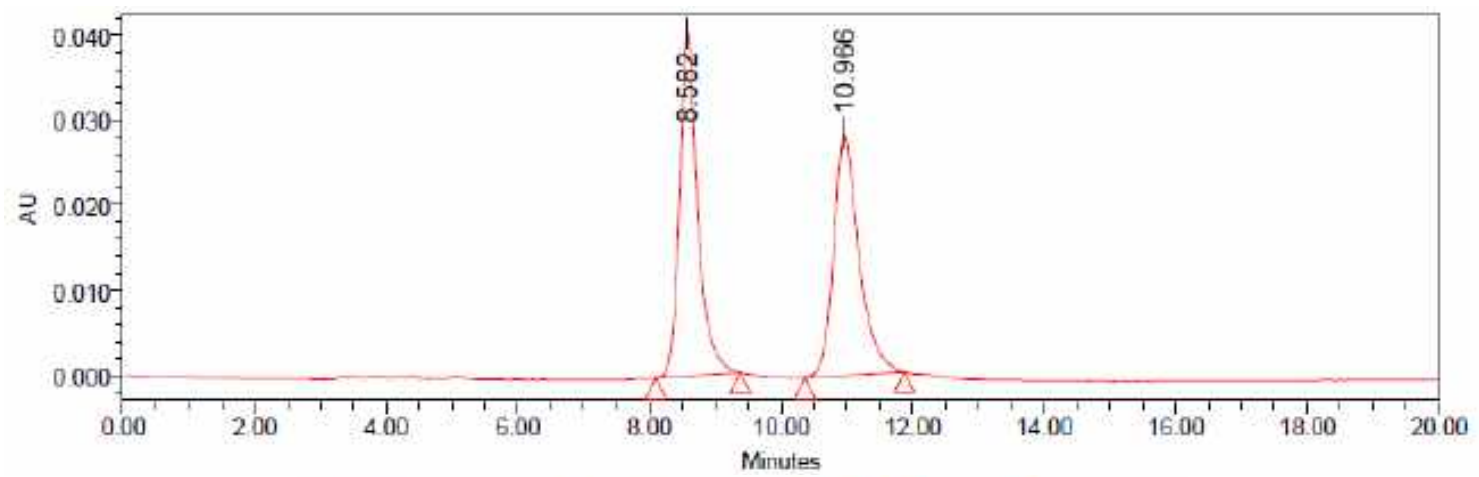

\section{Processed Channel: PDA $235.0 \mathrm{~nm}$}

\begin{tabular}{|l|l|r|c|r|r|}
\hline & Processed Channel & $\begin{array}{r}\text { Retention } \\
\text { Time }(\mathrm{min})\end{array}$ & Area & $\%$ Area & Height \\
\hline 1 & PDA $235.0 \mathrm{~nm}$ & 8.582 & 778543 & 50.69 & 40333 \\
\hline 2 & PDA $235.0 \mathrm{~nm}$ & 10.966 & 757293 & 49.31 & 28425 \\
\hline
\end{tabular}

\section{Enantioriched sample of $\left(R_{a}, R, R, S, R, S\right)-\mathbf{1 6} \mathbf{i}$ :}

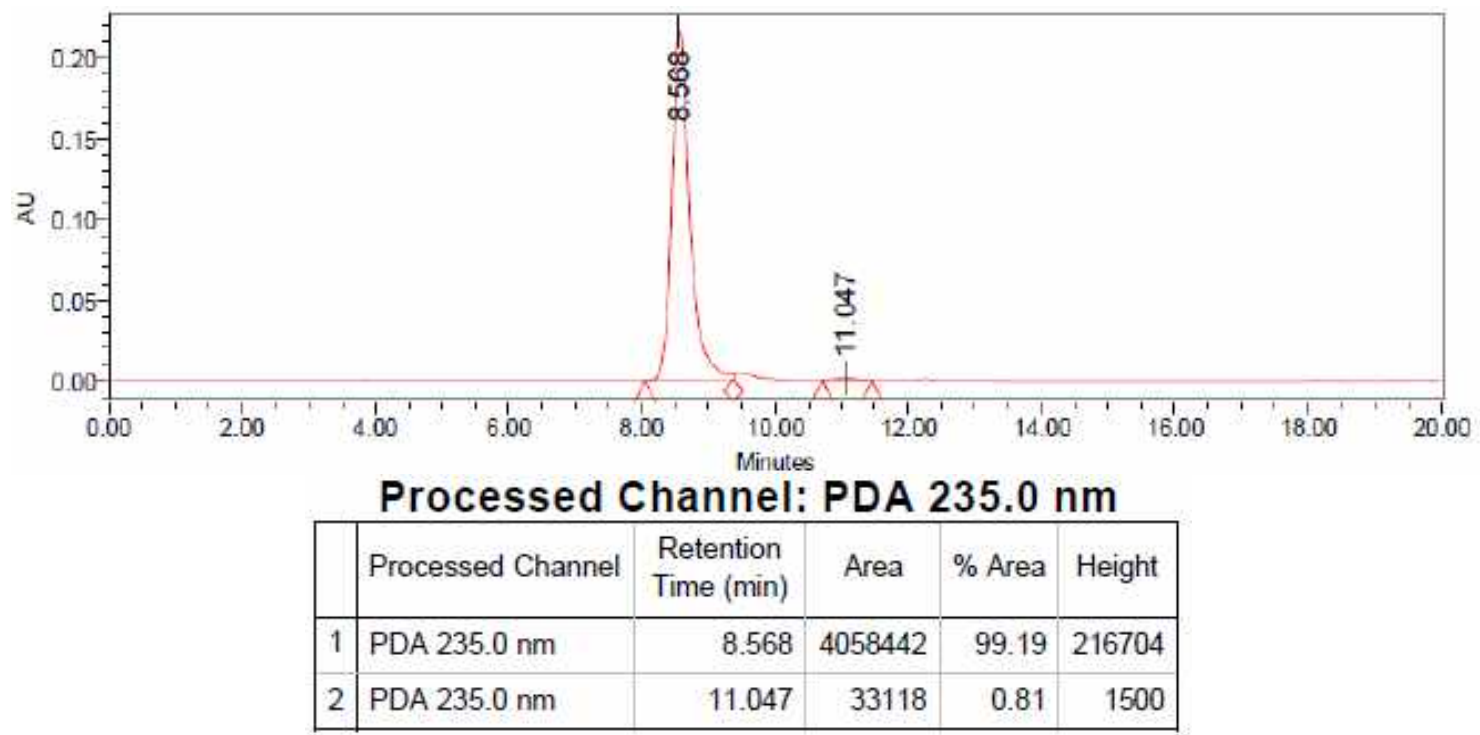


${ }^{1} \mathrm{H} \mathrm{NMR}\left(400 \mathrm{MHz}, \mathrm{CDCl}_{3}\right)$ of $\left(R_{a}, R, R, S\right)-\mathbf{1 1} \mathbf{h}:$

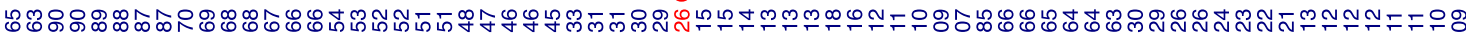

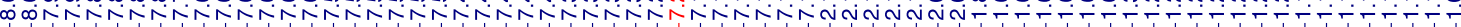

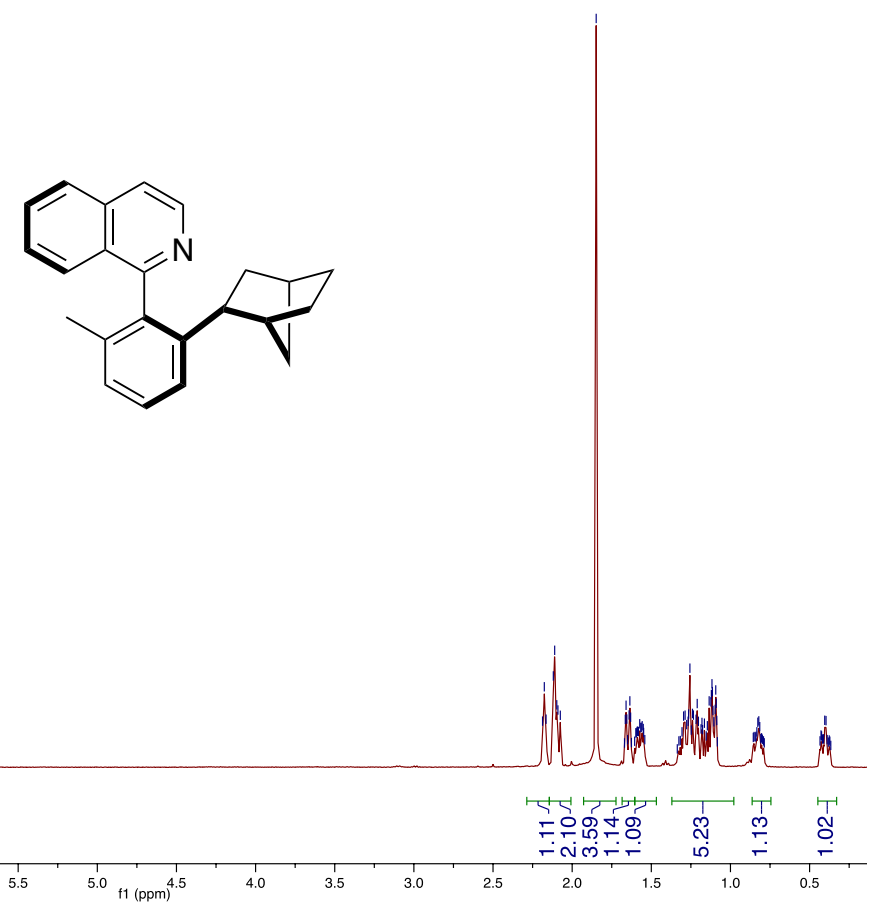

${ }^{13} \mathrm{C}$ NMR $\left(100 \mathrm{MHz}, \mathrm{CDCl}_{3}\right)$ of $\left(R_{a}, R, R, S\right)-\mathbf{1 1 h}$ :

定

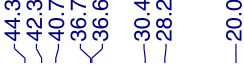
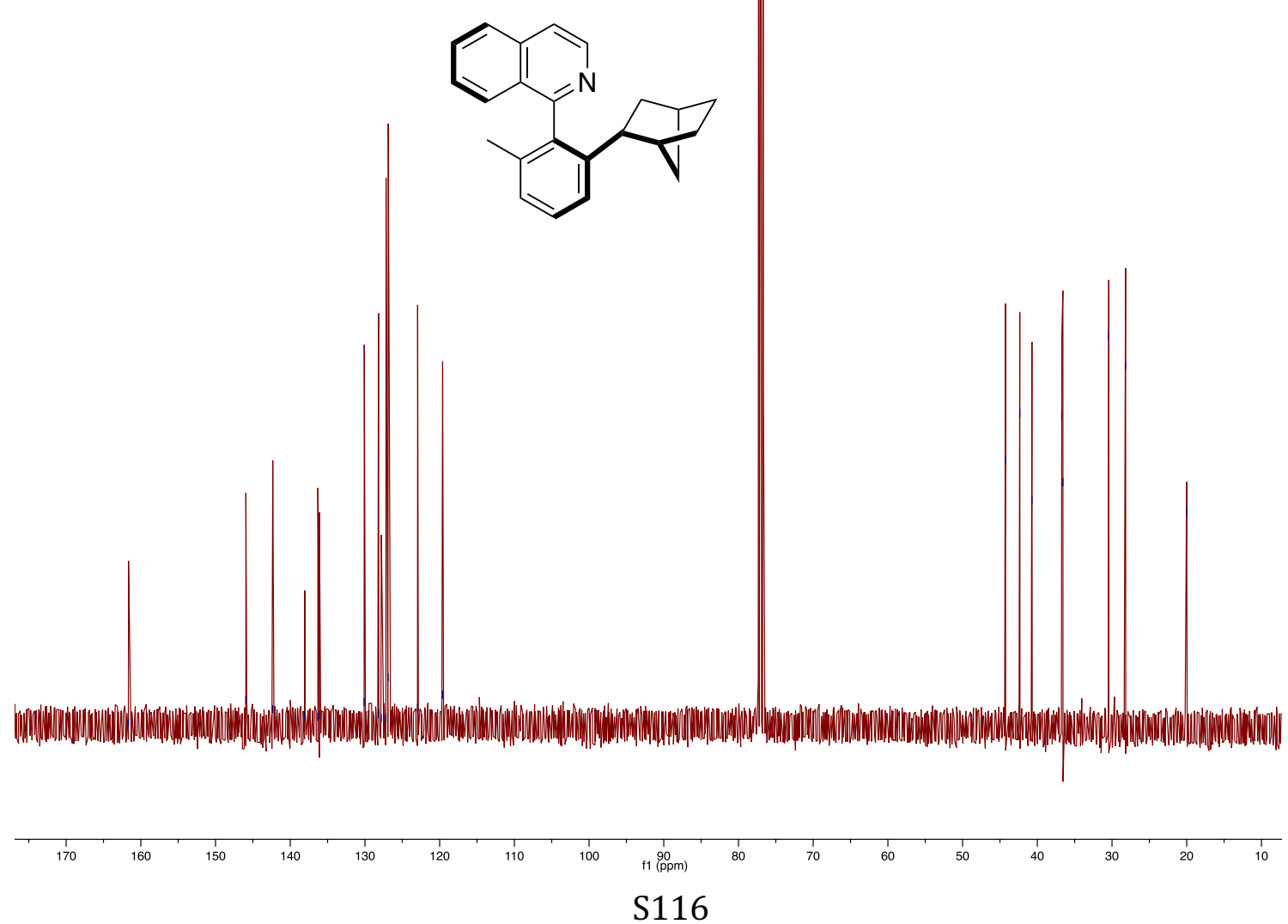
Racemic sample of $( \pm)$-11h: IG column, $n$-hexane:isopropanol 99:1, F= $1 \mathrm{~mL} / \mathrm{min}, \mathrm{T}=$ $30^{\circ} \mathrm{C}$.

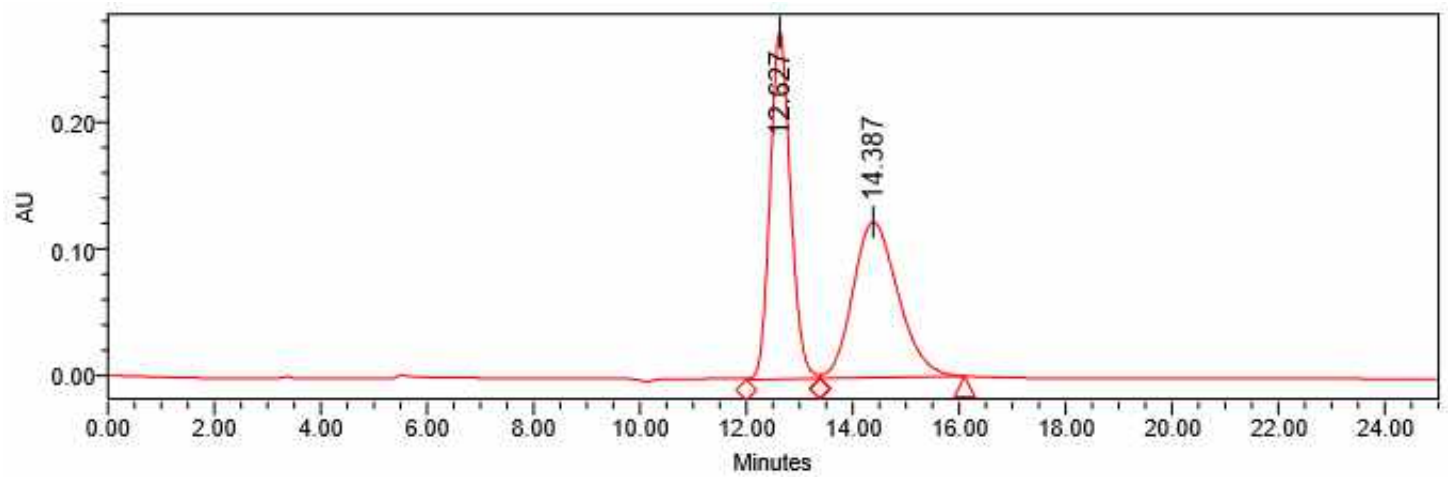

\section{Processed Channel: PDA $218.0 \mathrm{~nm}$}

\begin{tabular}{|r|l|r|c|r|r|}
\hline & Processed Channel & $\begin{array}{c}\text { Retention } \\
\text { Time (min) }\end{array}$ & Area & \% Area & Height \\
\hline 1 & PDA 218.0 nm & 12.627 & 7354194 & 50.28 & 274637 \\
\hline 2 & PDA $218.0 \mathrm{~nm}$ & 14.387 & 7272614 & 49.72 & 123100 \\
\hline
\end{tabular}

Enantioriched sample of $\left(R_{a}, R, R, S\right)$-11h:

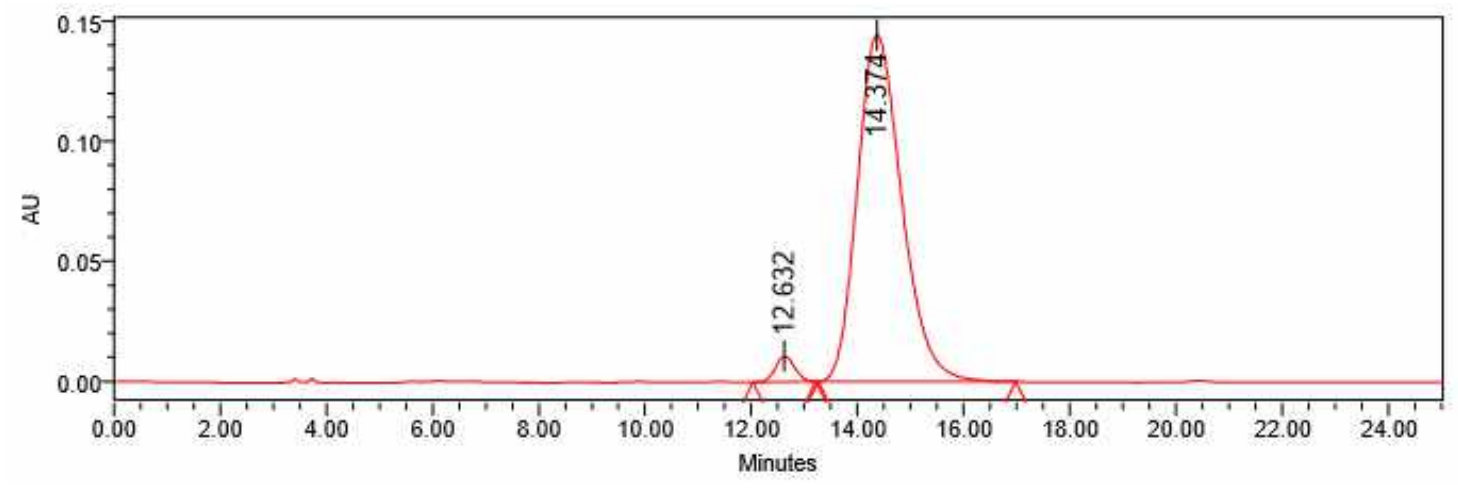

Processed Channel: PDA $218.0 \mathrm{~nm}$

\begin{tabular}{|l|l|r|r|r|r|}
\hline & Processed Channel & $\begin{array}{c}\text { Retention } \\
\text { Time (min) }\end{array}$ & Area & \% Area & Height \\
\hline 1 & PDA 218.0 nm & 12.632 & 270682 & 3.12 & 10676 \\
\hline 2 & PDA 218.0 nm & 14.374 & 8407202 & 96.88 & 144572 \\
\hline
\end{tabular}


${ }^{1} \mathrm{H}$ NMR $\left(400 \mathrm{MHz}, \mathrm{CDCl}_{3}\right)$ of $\left(R_{a}, R, R, S\right)-\mathbf{1 7} \mathbf{h}$ :

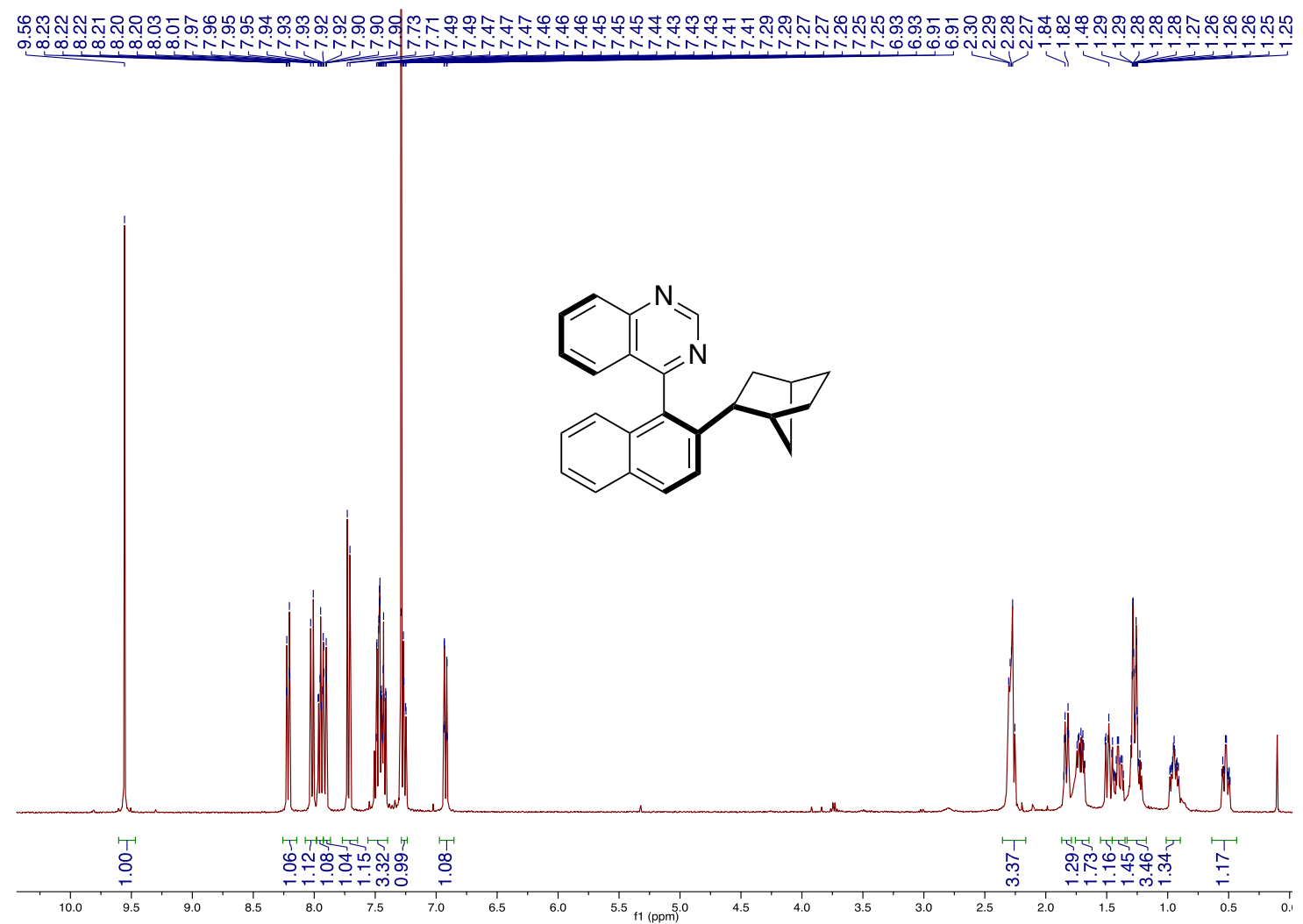

${ }^{13} \mathrm{C}$ NMR $\left(100 \mathrm{MHz}, \mathrm{CDCl}_{3}\right)$ of $\left(R_{a}, R, R, S\right)-\mathbf{1 7} \mathbf{h}$ :

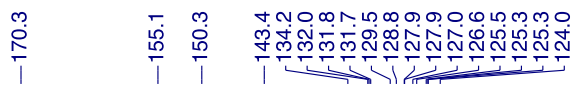

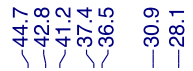

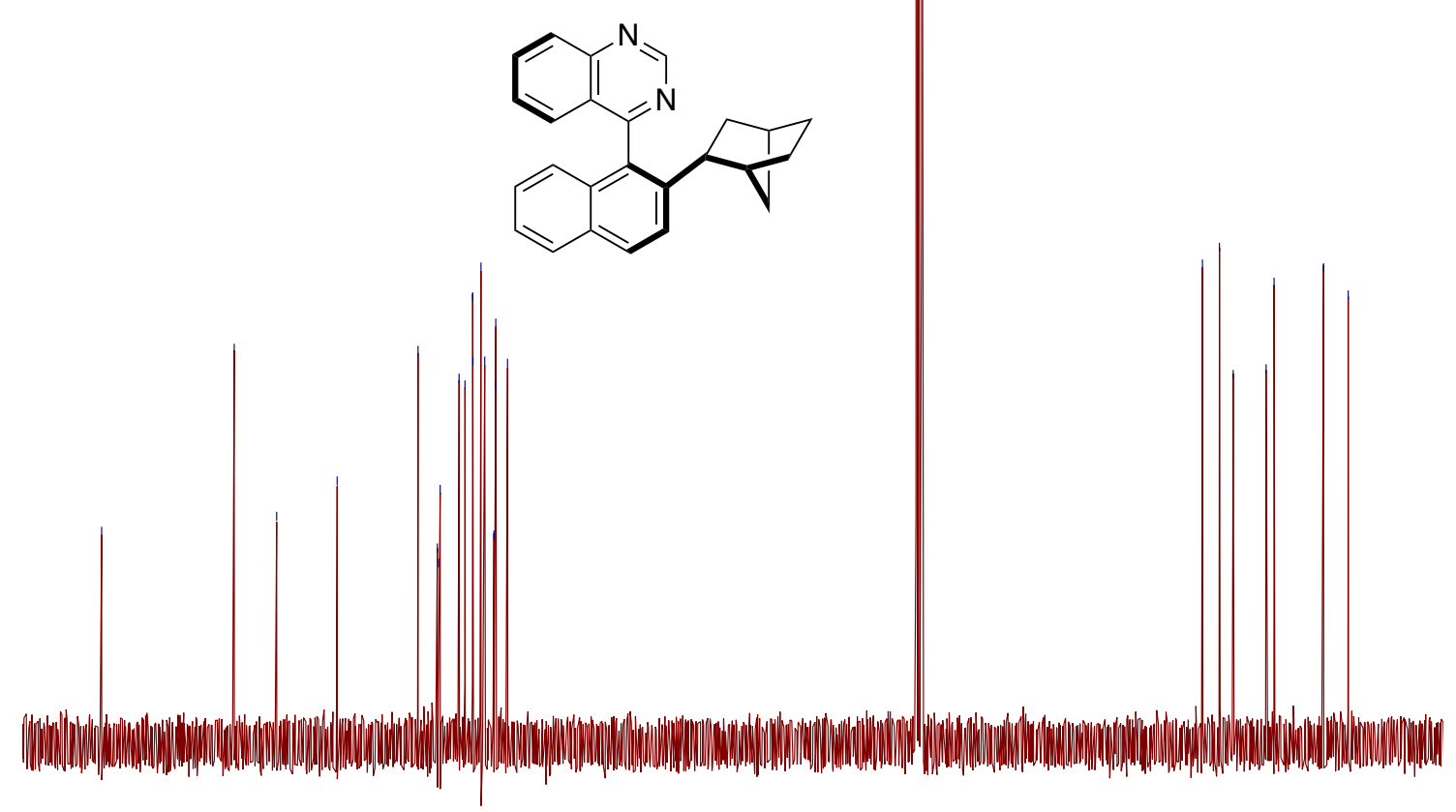

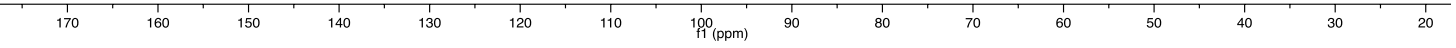


Racemic sample of $( \pm)$-17h: IA column, $n$-hexane:isopropanol 98:2, F= $1 \mathrm{~mL} / \mathrm{min}, \mathrm{T}=$ $30^{\circ} \mathrm{C}$.

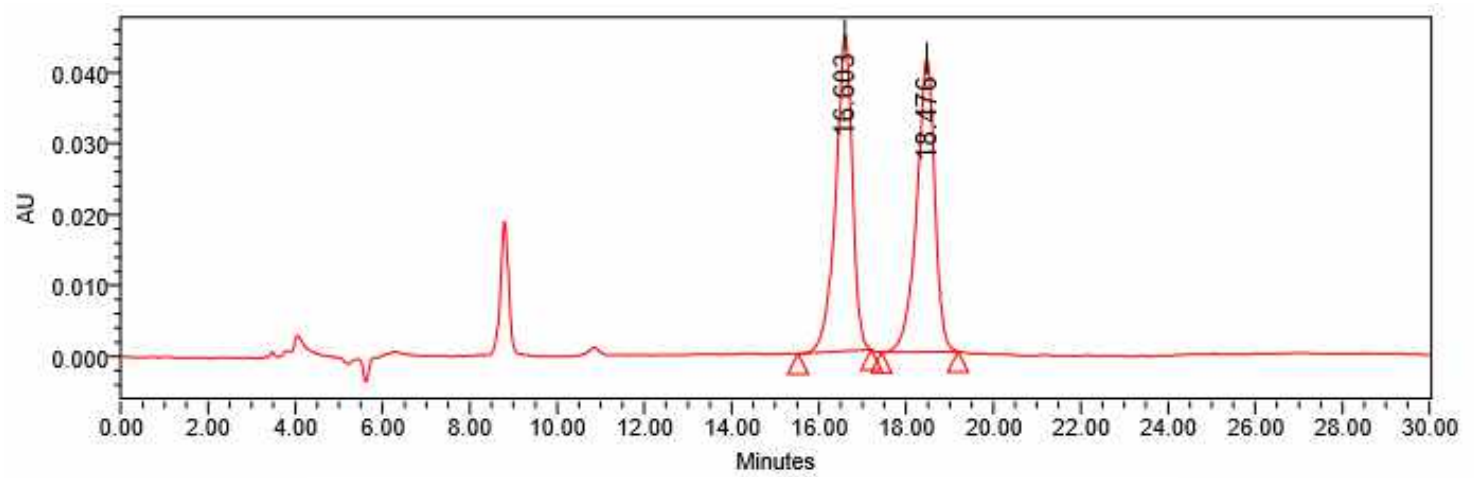

Processed Channel: PDA $227.0 \mathrm{~nm}$

\begin{tabular}{|l|l|r|r|r|r|}
\hline & Processed Channel & $\begin{array}{r}\text { Retention } \\
\text { Time }(\mathrm{min})\end{array}$ & Area & \% Area & Height \\
\hline 1 & PDA 227.0 nm & 16.603 & 1184168 & 49.88 & 44670 \\
\hline 2 & PDA 227.0 nm & 18.476 & 1189867 & 50.12 & 41376 \\
\hline
\end{tabular}

Enantioriched sample of $\left(R_{a}, R, R, S\right)$-17h:

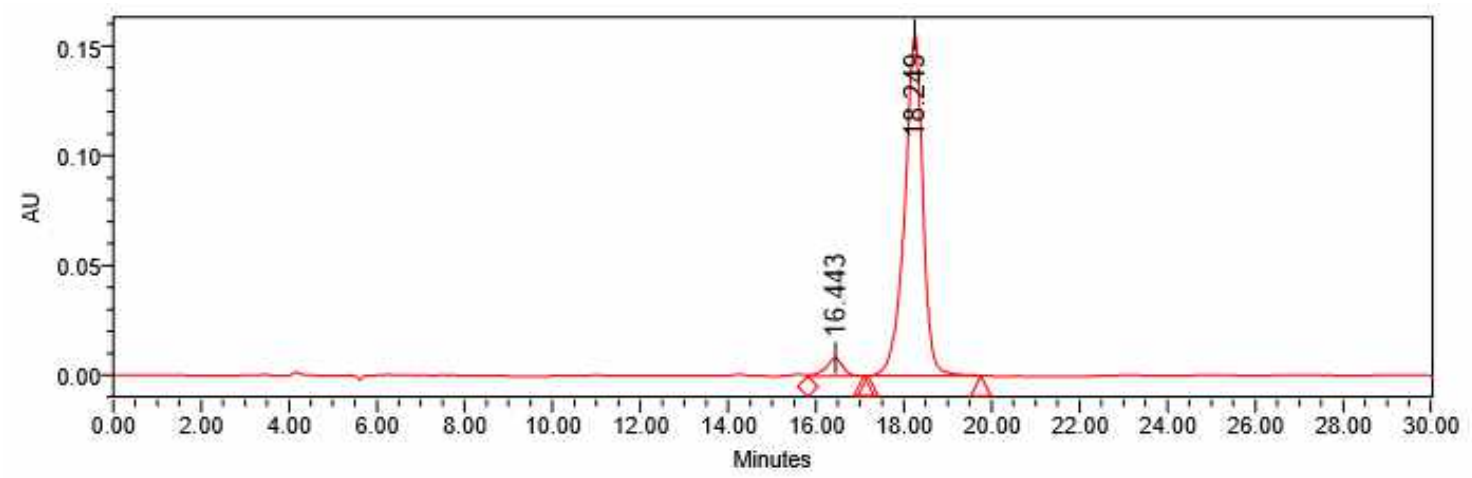

Processed Channel: PDA $227.0 \mathrm{~nm}$

\begin{tabular}{|r|l|r|r|r|r|}
\hline & Processed Channel & $\begin{array}{c}\text { Retention } \\
\text { Time (min) }\end{array}$ & Area & $\%$ Area & Height \\
\hline 1 & PDA $227.0 \mathrm{~nm}$ & 16.443 & 216255 & 4.61 & 8165 \\
\hline 2 & PDA $227.0 \mathrm{~nm}$ & 18.249 & 4478146 & 95.39 & 155516 \\
\hline
\end{tabular}

